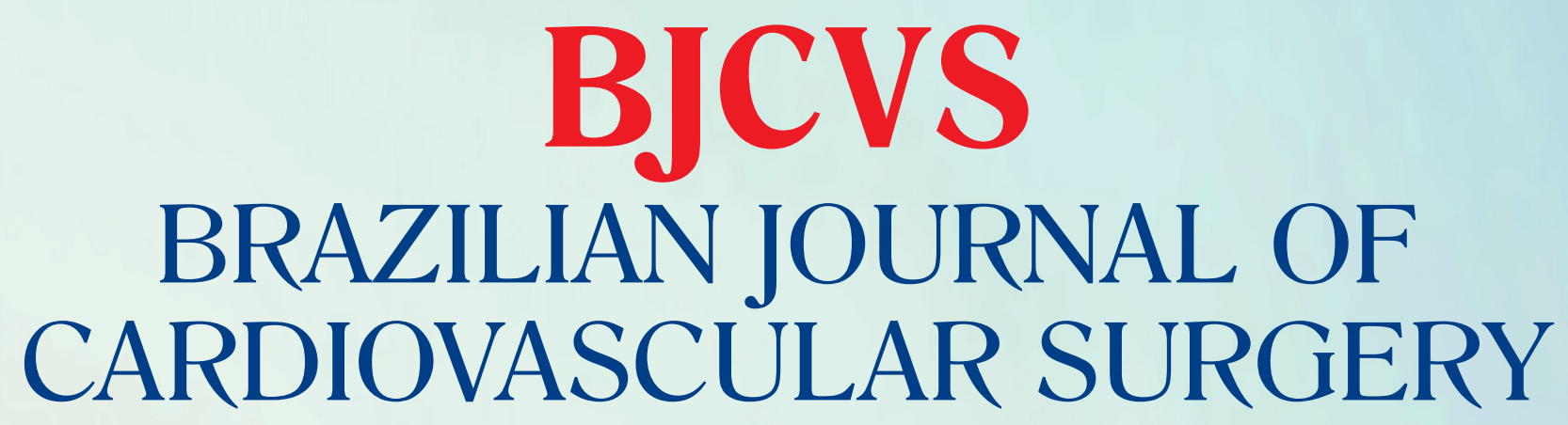

REVISTA BRASILEIRA DE CIRURGIA CARDIOVASCULAR

$44^{\text {th }}$ Congress of the Brazilian Society of Cardiovascular Sugery $44^{\circ}$ Congresso da Sociedade Brasileira de Cirurgia Cardiovascular April 20-22, 2017 - Rio de janeiro - RJ - Brazil

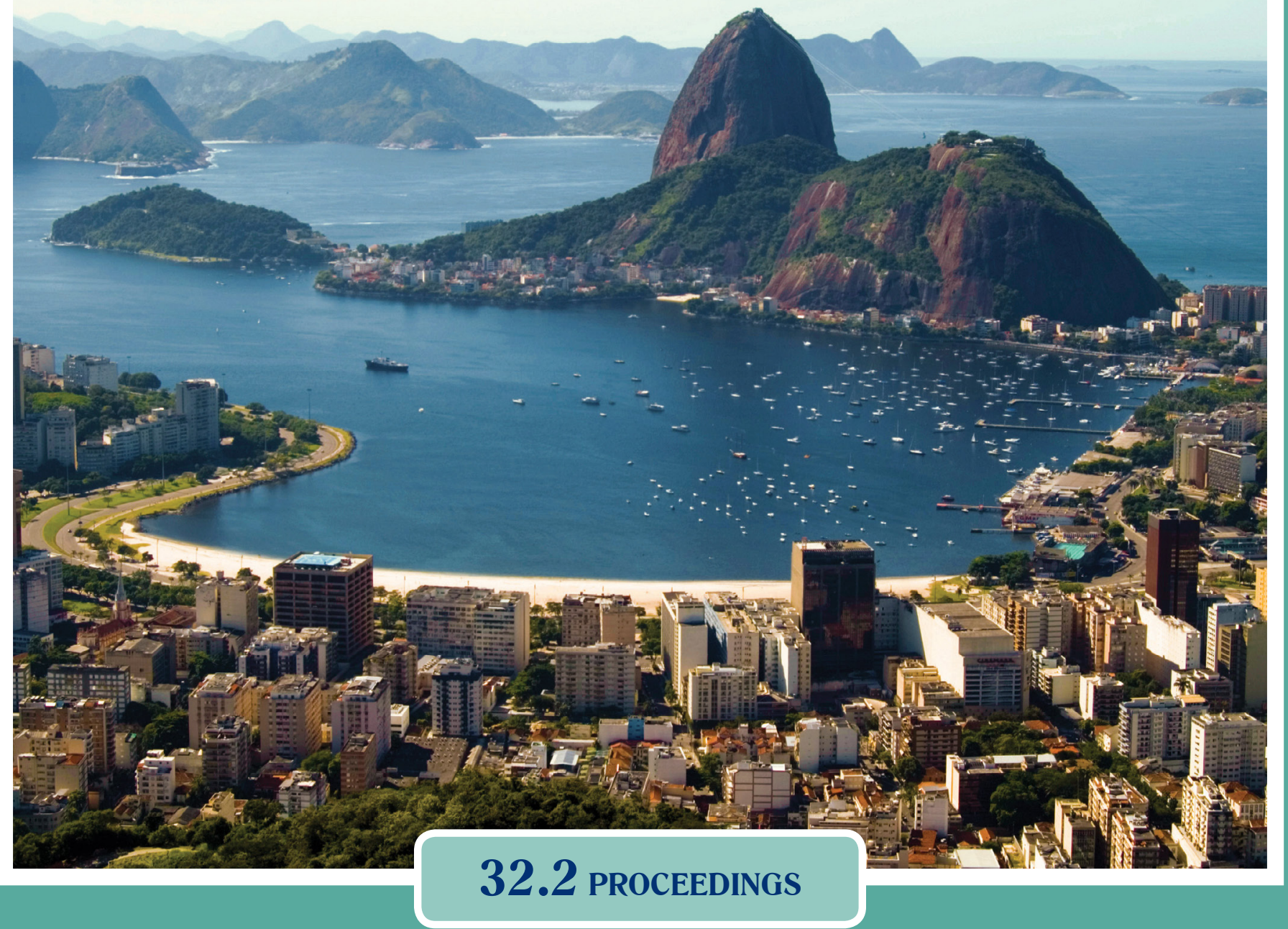





\title{
EDITOR-IN-CHIEF
}

\author{
Prof. Dr. Domingo M. Braile - PhD \\ São José do Rio Preto - SP - Brazil \\ domingo@braile.com.br
}

BRAZILIAN JOURNAL OF CARDIOVASCULAR SURGERY

\section{FORMER EDITORS}

- Prof. Dr. Adib D. Jatene

- Prof. Dr. Fábio B. Jatene
PhD - São Paulo (BRA) [1986-1996] PhD - São Paulo (BRA) [1996-2002]

\section{ASSOCIATE EDITORS}

ADVANCES IN CARDIOVASCULAR SURGERY

- Tomas A. Salerno - Miami (USA)

\section{AORTA}

- Eduardo Keller Saadi - Porto Alegre (BRA)

- Luciano Cabral Albuquerque - Porto Alegre (BRA)

\section{BASIC RESEARCH}

- Enio Buffolo - São Paulo (BRA)

- Paulo Roberto B. Évora - Ribeirão Preto (BRA)

\section{CARDIOLOGY}

- Moacir Fernandes Godoy

CARDIOVASCULAR REHABILITATION

- Solange Guizilini - São Paulo (BRA)

CARDIOVASCULAR SURGERY IN THE ADULT

- Marcela da Cunha Sales - Porto Alegre (BRA)

\section{CARDIOVASCULAR SURGERY}

- Henrique Murad - Rio de Janeiro (BRA)

- Adolfo Saadia

- Alan H. Menkis

- Alexandre Visconti Brick

- Antônio Sérgio Martins

- Bayard Gontijo Filho

- Borut Gersak

- Carlos Roberto Moraes

- Eduardo Sérgio Bastos

- Fábio Biscegli Jatene

- Fernando Antônio Lucchese

- Gianni D. Angelini

- Gilberto Venossi Barbosa

- Gilles D. Dreyfus

- Ivo A. Nesralla

- Jarbas J. Dinkhuysen

- José Antônio F. Ramires

- José Dario Frota Filho

- José Pedro da Silva

- José Teles de Mendonça

- Joseph A. Dearani

- Joseph S. Cosell

- Luis Alberto Oliveira Dallan
Buenos Aires (ARG) Winnipeg (CAN) Brasília (BRA) Botucatu (BRA) Belo Horizonte (BRA) Ljubljana (SLO) Recife (BRA) Rio de Janeiro (BRA) São Paulo (BRA) Porto Alegre (BRA) Bristol (UK) Porto Alegre (BRA) Harefield (UK) Porto Alegre (BRA) São Paulo (BRA) São Paulo (BRA) Porto Alegre (BRA) São Paulo (BRA) Aracaju (BRA) Rochester (USA) Houston (USA) São Paulo (BRA)

\section{EDITORIAL FELLOW}

- Gabriel Liguori - MD

São Paulo (BRA) - gabrielliguori@sbccv.org.br

\section{MANAGING EDITOR}

- Meryt Zanini - Postgraduate degree in Health Law from Faculdade de Saúde Pública da USP and MBA in Health Management from FGV São Paulo (BRA) - meryt@sbccv.org.br

\section{ENGLISH VERSION}

- Fernando Pires Buosi

- Renata Siqueira Campos

GRAPHIC DESIGN AND LAYOUT

- Silvia Seabra
CME

- João Carlos Ferreira Leal - São José do Rio Preto (BRA)

\section{CONGENITAL}

- Leonardo Augusto Miana - São Paulo (BRA)

- Orlando Petrucci Jr. - St. Louis (USA)

- Vinicius José Da Silva Nina - São Luís (BRA)

\section{CORONARY}

- Bruno Botelho Pinheiro - Goiânia (BRA)

- Walter José Gomes - São Paulo (BRA)

REGENERATIVE AND TRANSLATIONAL MEDICINE

- Paulo Roberto Slud Brofman - Curitiba (BRA)

\section{STATISTICS}

- Orlando Petrucci Jr. - Campinas (BRA)

- Michel Pompeu B. de O. Sá - Recife (BRA)

VALVE

- Manuel de Jesus Antunes - Coimbra (POR)

\section{EDITORIAL ASSISTANT}

- Camila Safadi - Postgraduate degree in Project Management - PMI S. José do Rio Preto (BRA) - camila@sbccv.org.br

MANUSCRIPT EDITING SPECIALIST

- Rosangela Monteiro - PhD

São Paulo (BRA)

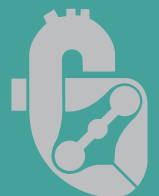




\section{SOCIEDADE BRASILEIRA DE CIRURGIA CARDIOVASCULAR}

\section{ADDRESS}

\section{SOCIEDADE BRASILEIRA DE CIRURGIA CARDIOVASCULAR}

Rua Afonso Celso, 1178 • Vila Mariana • Phone: 5511 3849-0341. Fax: 5511 5096-0079.

Zip code: 04119-061 - São Paulo, SP, Brazil

E-mail BJCVS: bjcvs@sbccv.org.br.E-mail SBCCV: sbccv@sbccv.org.br

- Site SBCCV: www.sbccv.org.br

- Sites BJCVS: www.scielo.br/rbccv • www.bjcvs.org • https://mc04.manuscriptcentral.com/rbccv-scielo (also for article submission)

- Thomson Scientific (ISI)

science.thomsonreuters.com

- PubMed Central

www.ncbi.nlm.nih.gov/pmc/

- PubMed/Medline

www.ncbi.nlm.nih.gov/sites/entrez

- SciELO - Scientific Library Online www.scielo.br

- Scopus (Scimago)

www.scimagojr.com

- Google scholar

scholar.google.com.br/scholar

\section{INDEXED IN}

- LATINDEX - Sistema Regional de Información en Línea para Revistas Cientificas de America Latina, el Caribe, España y Portugal

www.latindex.unam.mx

- LILACS - Literatura Latino-Americana e do Caribe em Ciências da Saúde.

lilacs.bvsalud.org/

- EBSCO

www2.ebsco.com/pt-br

- ProQuest

www.proquest.com

- Redalyc

www.redalyc.org

\section{SUPPORT}

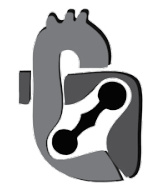

SOCIEDADE

BRASILEIRA DE

CIRURGIA

CARDIOVASCULAR

This journal is affiliated to

Associação Brasileira
de Editores Cientificos

A B E C

\section{Cc) creative}

All the contents of this journal, except where otherwise noted, is licensed under a Creative Commons Attribution License 


\title{
SOCIEDADE BRASILEIRA DE CIRURGIA CARDIOVASCULAR
}

\author{
DEPARTMENT OF SURGERY OF THE BRAZILIAN \\ SOCIETY OF CARDIOLOGY
}

"Enhancing the professional on behalf of the patient"

BOARD OF DIRECTORS 2016 - 2017

President:

Vice-President:

Secretary General:

Financial Director:

Scientific Director:

Education Director:

Advisory Board:

Journal Editor:

Events Director:

Site Editor:

Newsletter Editors:
Fabio Biscegli Jatene (SP)

Rui M. S. S. A. Almeida (PR)

Bruno Botelho Pinheiro (GO)

Eduardo Augusto Victor Rocha (MG)

Renato Abdala Karam Kalil (RS)

Henrique Murad (RJ)

Luciano Cabral Albuquerque (RS)

João Carlos Ferreira Leal (SP)

Josalmir Jose Melo do Amaral (RN)

Marcelo Sávio da Silva Martins (RJ)

Ricardo Carvalho Lima (PE)

Domingo Marcolino Braile (SP)

Carlos Manuel de Almeida Brandão (SP)

Melchior Luiz Lima (ES)

Domingo Marcolino Braile (SP)

Fernando Ribeiro Moraes Neto (PE)

Luciano Cabral Albuquerque (RS)

Orlando Petrucci (USA)

Walter José Gomes (SP)

Mariano Brasil Terrazas (AM)

Marcelo Sávio da Silva Martins (RJ)

Marcelo Biscegli Jatene (SP)

Luiz Cláudio Moreira Lima (MG)

Wilson Luiz da Silveira (GO)

Luciano Cabral Albuquerque (RS)

Andrea Dumsch de Aragon Ferreira (PR)

Milton de Miranda Santoro (SC)

Fernando Ribeiro de Moraes Neto (PE)

Fernando Antibas Atik (DF)

Luiz Paulo Rangel Gomes da Silva (PA)

João Carlos Ferreira Leal (SP)

José Carlos Dorsa V. Pontes (MS)

Ricardo Adala Benfatti (MS)

Nathalia Ribeiro (CE)

Eduardo Nunes Toniasso (SP)

Sintya Tertuliano Chalegre (PE) 

Com imenso prazer apresento aos leitores o Programa Oficial do $44^{\circ}$ Congresso da Sociedade Brasileira de Cirurgia Cardiovascular, a realizar-se de 20 a 21 de abril de 2017, no "Riocentro Exhibition \& Convention Center in Rio de Janeiro, RJ". Além da programação científica, têm sido agregadas inovações tornando o evento cada vez mais completo, nada a devendo aos encontros internacionais.

O tradicional coquetel de abertura este ano será oferecido no estande da SBCCV e BJCVS, no dia 20 de abril, às 19h00, no qual teremos o pré-lançamento do livro "Cardiovascular Surgery - A Clinical Casebook" da Liga acadêmica da SBCCV.

Como nos anos anteriores, este Suplemento do BJCVS traz as atividades do Congresso (agenda, programação científica, resumos dos Temas Livres e Pôsteres).

Este ano, o tema será "Alta Tecnologia: Conhecer mais para empregar melhor".

Paralelamente, serão realizados o 70 Simpósio de Enfermagem em Cirurgia Cardiovascular, o 70 Simpósio de Fisioterapia em Cirurgia Cardiovascular e o $6^{\circ}$ Congresso Acadêmico em Cirurgia Cardiovascular, o $35^{\circ}$ Congresso de Brasileiro de Circulação Extracorpórea, o Simpósio das Mulheres Cirurgiãs, e o Encontro dos Residentes.

Sempre no intuito de discutir os mais recentes avanços nas técnicas cirúrgicas e a incorporação de novas tecnologias, teremos importantes convidados internacionais.

Estarão presentes os doutores Hartzell Schaff, Mayo Clinic (EUA); John D. Puskas, Mount Sinai (EUA); Eric Velasquez, Duke University (EUA); Andreas Martens, Hannover Medical School (Alemanha); Michael Madani, University of California San Diego (EUA); Emir Kabil, Centre for heart Tuzla (BIH) e Vlassis Ninios, Interventional Cardiologist, St. Luke's Hospital (GRE).

O tradicional "Hands-On" será realizado no dia 20 (quinta-feira), com dois blocos, divididos em quatro módulos.

A grande novidade, da grade do $44^{\circ}$ Congresso da SBCCV, é o curso de Metodologia Científica que acontecerá no dia 20 de abril e os cursos de TAVI, TEVAR, Cirurgia da Hipertensão Pulmonar PR Tromboembolismo Crônico (HPTEC) e Curso Ecocardiografia Intraoperatória que acontecerão no dia 22 de abril, trazendo grandes profissionais para compartilhar seus conhecimentos.

Os Temas Livres esse ano terão uma premiação diferenciada.

A SBCCV implementou vários projetos educacionais entre eles a premiação dos temas livres do congresso para o 10 LugarPassagem aérea e Inscrição em Congresso Internacional (AATS, STS ou EACTS), 2o Lugar- Passagem aérea e Inscrição no próximo Congresso SBCCV e 30 Lugar - Inscrição no próximo Congresso SBCCV. Esses projetos objetivam contribuir como estimulo à produção científica na especialidade.

É uma ótima oportunidade para aprimorarem os conhecimentos com a orientação de professores experientes.

Saúdo a Comissão Organizadora, coordenada pelo Dr. Henrique Murad, pela dedicação impar na viabilização da logística e auxílio na programação científica, responsáveis pela atração do grande número de participantes que se deslocaram até Rio de Janeiro, desta forma engrandecendo cada vez mais o nosso Congresso.
Não posso me esquecer também das empresas que estarão expondo seus produtos, tão importantes para o desempenho das nossas atividades indispensáveis para tornar realidade o $44^{\circ}$ Congresso da SBCCV.

Agradeço a confiança e a parceria que se mantêm, mesmo no atual cenário econômico de muitas incertezas.

No dia 21 de abril, das 13 h00 às 14h00, na Sala 211, ocorrerá a

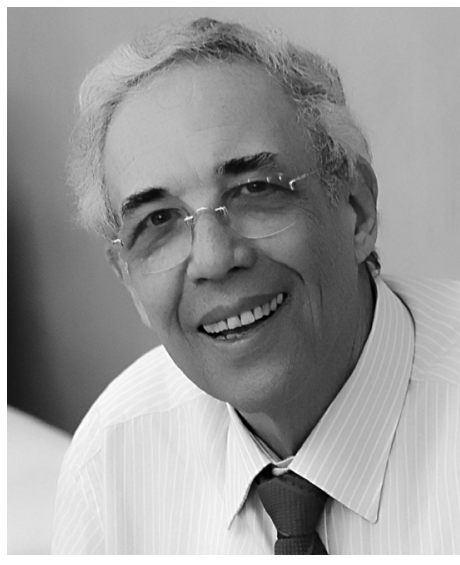
reunião do Corpo Editorial do BJCVS com os Editores Associados, Membros do Conselho Editorial, Revisores e sempre aberta a todos os sócios.

Serão discutidos os rumos da revista, prestação de contas (financeiro), prestação de contas (científico), sistema ScholarOne - análise de 8 meses de novo sistema. O que mudou? editores Associados e Revisores - apresentação do fluxo de trabalho dos editores por área e discussão para implementação de plano de ação em relação ao Publons, ABEC, Plágio, CrossRef e DOI, ORCID, Fator de Impacto do BJCVS - campanha, mudança para o nome Brazilian Journal of Cardiovascular Surgery, planejamento 2017/2018 (Projeção financeira e científica) para compartilhar as novidades e também como forma de agregar mais leitores e atrair artigos de boa qualidade.

Lembro que o BJCVS pode ser acessada nos sites www.bjcvs.org e www.scielo.br/rbccv, além de todas as Bases Internacionais como Medline-Pubmed, PubMed Central, Thomson Reuters, Scopus, Google Scholar, etc. O conteúdo completo de todas as edições, desde 1986, está disponível, nos diferentes sites sem qualquer custo para os leitores "Free Access" em todas as plataformas.

Também podem ser baixados aplicativos específicos da Revista para smartphones ou Tablets nos sistemas operacionais ANDROID ou IOS, bastando buscar os APPs pela sigla: BJCVS na Play Store, Google Play ou na Apple Store.

Espero que os participantes possam usufruir da programação e aproveitar os momentos de lazer e confraternização no Rio de Janeiro, conhecida pelas suas belezas naturais, hospitalidade, cidade do samba e da alegria.

Agradeço a confiança da Diretoria da SBCCV e da Comissão Organizadora, pelo irrestrito apoio que recebemos ao longo dos anos.

Finalizo, lembrando que o BJCVS continua aberto para publicar Trabalhos Científicos, Cartas e Editoriais, em inglês.

Recebam meu caloroso abraço

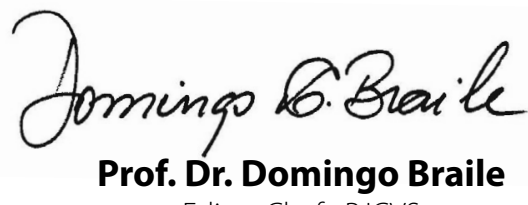

Editor-Chefe BJCVS 



\section{Sumário}

\begin{tabular}{|c|c|}
\hline Mensagem da Comissão Organizadora & 8 \\
\hline Comissões & 9 \\
\hline Agradecimentos Especiais & 12 \\
\hline Informações do evento & 13 \\
\hline Local do evento & 14 \\
\hline Agenda: Reuniões, Provas e Assembleias & 16 \\
\hline Atividades do Congresso & 17 \\
\hline Programa Científico Congresso & 18 \\
\hline Programa Hands On e Arenas & 50 \\
\hline Simpósios Satélites & 51 \\
\hline \multicolumn{2}{|l|}{ Resumos dos Temas Livres e Pôsteres } \\
\hline Temas Livres • SBCCV & 54 \\
\hline Apresentação de Pôsteres SBCCV & 68 \\
\hline Exposição de Pôsteres SBCCV & 77 \\
\hline Temas Livres Acadêmicos & 114 \\
\hline Exposição de Pôsteres Acadêmicos & 120 \\
\hline Temas Livres Pediatria & 126 \\
\hline Temas Livres Fisioterapia & 132 \\
\hline Pôsteres Apresentados Fisioterapia & 140 \\
\hline Temas Livres Enfermagem & 150 \\
\hline Exposição de Pôsteres Enfermagem & 154 \\
\hline Temas Livres SBCEC & 158 \\
\hline Pôsteres SBCEC & 162 \\
\hline
\end{tabular}




\section{Mensagem da \\ Comissào Organizadora}

Caros amigos,

O Rio de Janeiro sediará o 44o Congresso da Sociedade Brasileira de Cirurgia Cardiovascular, de 20 a 22 de abril de 2017, no Riocentro. Esta é a terceira vez que a cidade sedia nosso Congresso (a última vez foi há dezesseis anos).

Será um ótimo momento para se atualizar, rever amigos, matar saudades, procurar oportunidades de trabalho e desfrutar desta cidade, que é e sempre será maravilhosa.

O Riocentro foi repaginado para as Olimpíadas e está pronto para nos receber. Em seu entorno há bons hotéis, shopping centers, áreas de lazer, uma vista sensacional e a sempre convidativa praia.

O tema do Congresso este ano é "Alta tecnologia. Conhecer mais para empregar melhor" e a Comissão Cientifica trabalhou com afinco para nos oferecer um programa realmente sensacional. Contaremos com a participação de renomados cirurgiões estrangeiros: Hartzell Schaff, Mayo Clinic (EUA); John D. Puskas, Mount Sinai (EUA); Eric Velasquez, Duke University (EUA); Andreas Martens, Hannover Medical School (Alemanha); Michael Madani, University of California San Diego (EUA); Emir Kabil, Centre for heart Tuzla (BIH) e Vlassis Ninios, St. Luke's Hospital (GRE).

Os jovens cirurgiões, as mulheres cirurgiãs e os residentes, assim como as ligas acadêmicas, terão um lugar especial neste congresso.

A integração multiprofissional também está na grade do Congresso, privilegiando enfermeiras, fisioterapeutas e perfusionistas.

Teremos amplas oportunidades para aprender o que há de mais moderno em cirurgia cardiovascular. Poderemos discutir condutas, trocar ideias e apresentar novidades. O hands-on, grande sucesso dos congressos anteriores, terá mais um desempenho espetacular.

A Comissão Organizadora está se sentindo como um grupo de atores às vésperas da primeira apresentação de uma nova peça. Estamos tensos, mas cientes de que faremos um belo espetáculo.

Sejam todos bem-vindos!

Vocês é que farão deste Congresso um evento memorável.

Comissão Organizadora do $44^{\circ}$ Congresso da SBCCV 

BRAZILIAN SOCIETY OF CARDIOVASCULAR SURGERY

\title{
COORDENADOR - 44० CONGRESSO DA SBCCV
}

Henrique Murad (RJ)

\section{COMISSÃO LOCAL}

Andrey José de Oliveira Monteiro

Marcelo Sávio da Silva Martins

Mário Ricardo Amar

Olivio Alves Souza Neto

\section{COMITÊ DE CONSULTORES CIENTÍFICOS}

\author{
Alfredo I Fiorelli (SP) \\ Bayard Gontijo Filho (MG) \\ Cezar Augusto Conforti (SP) \\ Diego Felipe Gaia dos Santos (SP) \\ Eduardo K. Saadi (RS) \\ Fábio Antonio Gaiotto (SP) \\ Fernando Antonio Fantini (MG) \\ Fernando Ribeiro Moraes Neto (PE) \\ Fernando Antibas Atik (DF) \\ Francisco D. A. Costa (PR) \\ Francisco Gregori Jr (PR) \\ Henrique Murad (RJ) \\ Januário Manuel de Souza (SP) \\ João Carlos F. Leal (SP) \\ João Jazbik Neto (MT) \\ José Teles de Mendonça (SE) \\ José Wanderley Neto (AL) \\ Leonardo Ferber Drumond (MG) \\ Luiz César Guarita Souza (PR) \\ Luiz Cláudio Moreira Lima (MG) \\ Luiz Felipe P. Moreira (SP) \\ Luiz Paulo Rangel G. da Silva (PA) \\ Magaly Arrais dos Santos (SP) \\ Marcela Cunha Sales (RS) \\ Noedir A. G. Stolf (SP) \\ Paulo Roberto S. Brofman (PR) \\ Rodrigo Mussi Milani (PR) \\ Sérgio Almeida de Oliveira (SP) \\ Vinicius José da S. Nina (MA) \\ Walter José Gomes (SP) \\ Walter Villela de A. Vicente (SP)
}




\title{
Comissões
}

COMISSÃO JULGADORA DE TEMAS LIVRES

44० CONGRESSO DA SBCCV

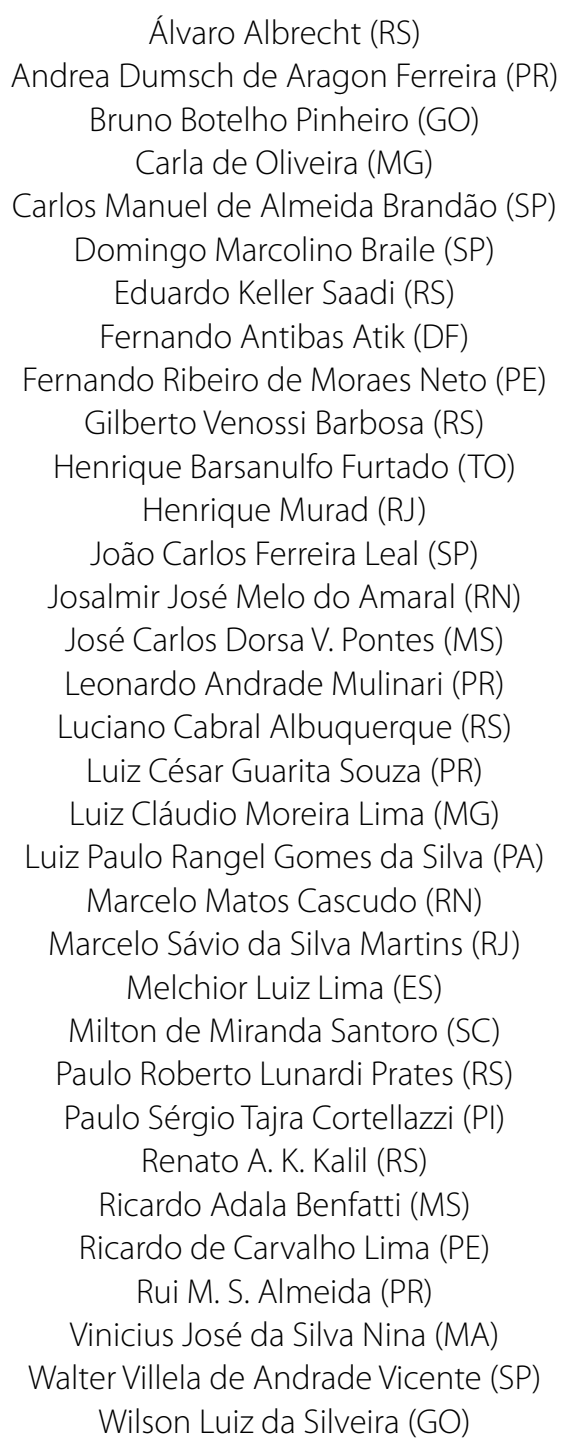

\section{COMISSÃO JULGADORA DE TEMAS LIVRES} $6^{\circ}$ CONGRESSO ACADÊMICO EM CIRURGIA CARDIOVASCULAR

\author{
Henrique Murad (RJ) \\ Nathalia Ribeiro Pinho de Sousa (CE) \\ Rubens Tofano de Barros (SP) \\ Rui M. S. Almeida (PR) \\ Walter José Gomes (SP)
}

\section{COMISSÃO NACIONAL HANDS-ON}

Gilberto Venossi Barbosa (RS)

Carlos Manuel de A. Brandão (SP)

Vinicius José da S. Nina (MA)

Milton de Miranda Santoro (SC)

Olívio A. de Souza Neto (RJ) 


\title{
COMISSÃO NACIONAL \\ Tereza Cristina Felippe Guimarães (RJ) \\ Eneida Rejane Rabelo da Silva (RS) \\ Etinéia Lino (DF) \\ Maria Antonieta Pereira de Moraes (RS)
} 7० SIMPÓSIO DE ENFERMAGEM EM CIRURGIA CARDIOVASCULAR

\section{COMISSÃO LOCAL}

7० SIMPÓSIO DE ENFERMAGEM EM CIRURGIA CARDIOVASCULAR

Carla Cristina Lima

Claudia Elizabeth de Almeida

Deyse Santoro

Lilian Bering

Iza Cristina dos Santos

\section{COMISSÃO JULGADORA DE TEMAS LIVRES \\ 7० SIMPÓSIO DE ENFERMAGEM EM CIRURGIA CARDIOVASCULA $R$}

\author{
Ana Carla Dantas Cavalcanti (RJ) \\ Eneida Rejane Rabelo da Silva (RS) \\ Etinéia Lino (DF) \\ Maria Antonieta Pereira de Moraes (RS)
}

\section{COMISSÃO NACIONAL \\ 7० SIMPÓSIO DE FISIOTERAPIA EM CIRURGIA CARDIOVASCULAR}

\author{
Michel Silva Reis (RJ) \\ Rodrigo B. Jaenisch (RS) \\ Solange Guizini (SP) \\ Valeria Papa (SP) \\ Vera Lucia dos Santos Alves (SP)
}

\section{COMISSÃO JULGADORA DE TEMAS LIVRES}

7० SIMPÓSIO DE FISIOTERAPIA EM CIRURGIA CARDIOVASCULAR

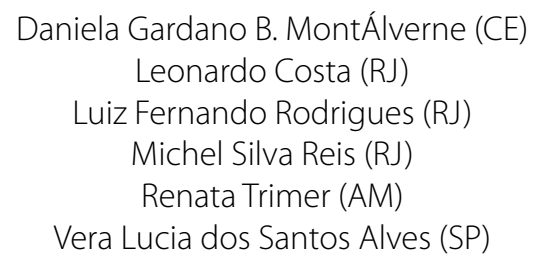




\section{Agradecimento Especial}

\section{Nosso agradecimento}

A Diretoria da SBCCV agradece o trabalho incansável da Comissão Organizadora do $44^{\circ}$ Congresso. Não foram medidos esforços para que o Congresso seja do mais alto nível de qualidade e proporcione bem-estar a todos os participantes.

Agradecemos, também, a participação das empresas expositoras e patrocinadoras, e órgãos de apoio, que ano após ano, permitem que os Congressos da SBCCV sejam um verdadeiro sucesso.

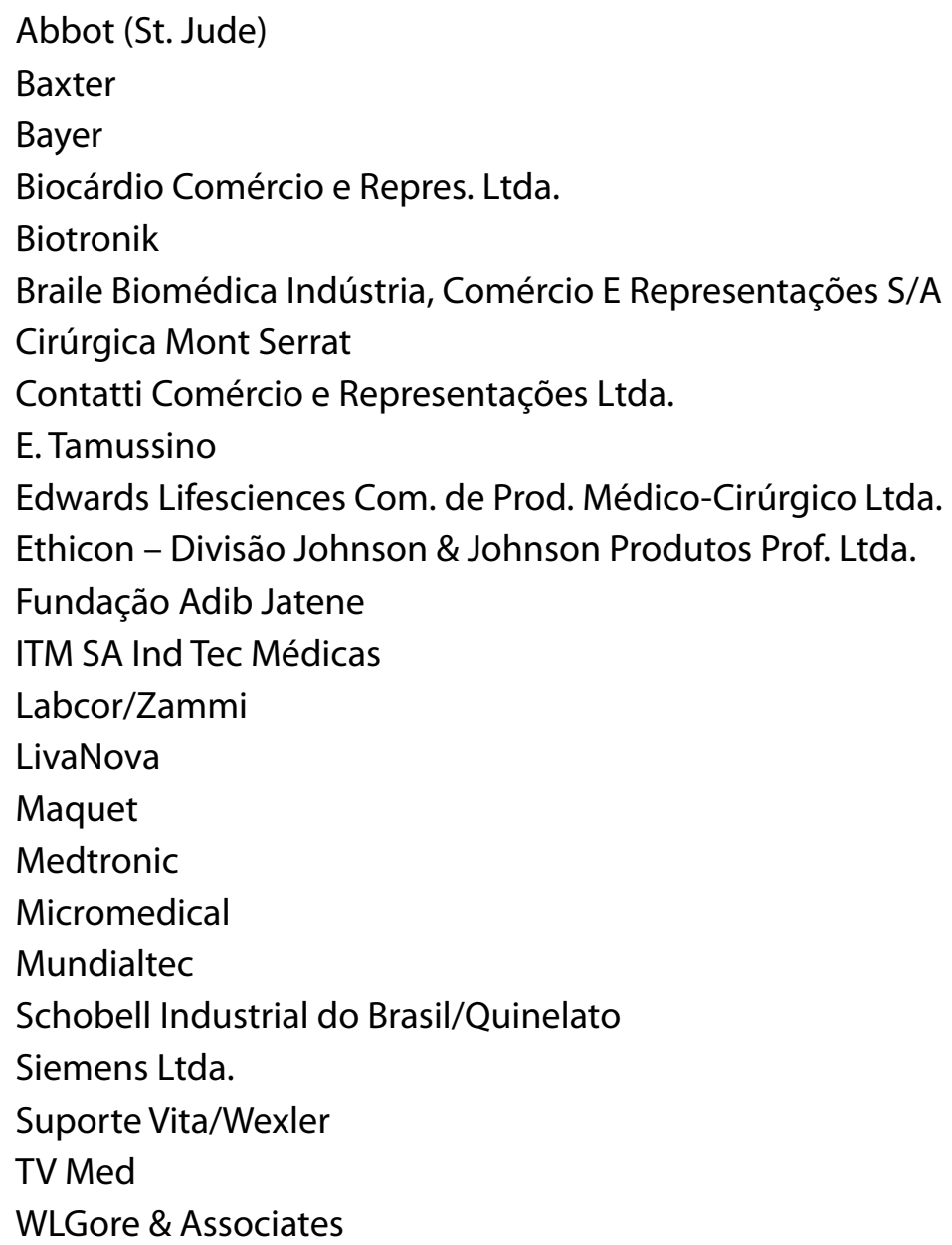

\section{ORGÃOS DE APOIO}

CAPES

FAPERJ 


\section{Seja bem-vindo}

\section{ABERTURA}

\section{DE ABRIL • QUINTA-FEIRA}

\section{ABERTURA}

Horário: $16 \mathrm{~h} 30$

Local: Auditório 1

\section{COQUETEL}

Horário: $19 \mathrm{~h}$

Local: Lounge SBCCV, área da exposição

\section{HORÁRIOS DE SECRETARIA \\ 20/04 - Quinta-feira - 7h/19h \\ 21/04 - Sexta-feira - 7h/19h \\ 22/04 - Sábado - 7h/14h \\ CRACHÁ DEACESSO}

O crachá entregue aos participantes é de uso obrigatório para o ingresso em todas as atividades, na área de exposição.

Evite perdê-lo ou esquecê-lo. Será cobrada uma taxa para confecção da segunda via do crachá.

\section{CERTIFCADOS}

Os certificados de participação serão disponibilizados em formato on line a partir do dia 28 de abril, no site do congresso: www. sbccv.org.br/44congresso

Os certificados de Temas Livres e Palestrantes serão enviados pela SBCCV.

\section{ÁREA DE EXPOSICÃO}

A exposição de produtos e serviços será, novamente, um sucesso.

Nos intervalos das atividades científicas aproveite para conhecer as novidades tecnológicas oferecidas pelas empresas que engrandecem a atuação profissional dos cirurgiões brasileiros.

\section{PROVA DO DECA}

\section{DE ABRIL • QUINTA-FEIRA}

Horário: $8 \mathrm{~h} / 13 \mathrm{~h}$

Local: Auditório 10

\section{PROVA SBCEC}

\section{DE ABRIL • SÁBADO}

Horário: $13 \mathrm{~h} / 16 \mathrm{~h}$

Local: Auditório 10

\section{LOUNGESBCCV}

Neste espaço, está localizado o web point para navegação gratuita na internet.

\section{ASSEMBLEIA SBCCV}

\section{DE ABRIL • SEXTA-FEIRA}

Horário: $18 \mathrm{~h} 15$

Local: Auditório 1

Esperamos os sócios para Assembleia Ordinária

\section{TEMAS LTVRES}

Os resumos dos Temas livres e Pôsteres aprovados para apresentação no Congresso estão disponíveis no BJCVS - Brazilian Journal of Cardiovascular Surgery Link para acesso: www.bjcvs.org. A Secretaria de Temas Livres está localizada junto à área de exposição de pôsteres para atender aos membros das comissões avaliadoras e apresentadores.

Confira os horários de sua apresentação na Secretaria de Temas Livres.

\section{ALIMENTACÃO \\ ALMOÇO (sistema de adesão)} Indicamos o Restaurante localizado no pavilhão 5 - térreo HORÁRIO DE ATENDIMENTO $12 \mathrm{~h}$ às $15 \mathrm{~h}$, durante os dias 20 e 21 de abril

VALOR:

Preço no local: $\mathrm{R} \$ 60,00$ /pessoa

\section{BLACK COFFEE:}

Disponível durante a realização do Evento - estrutura de cafeteria (cafés e lanches)

Localização - foyer do credenciamento

\section{APP PROGRAMACÃO CIENTÍFICA}

1. Baixe o aplicativo SBCCV pela Apple Store ou Google Play.

2. Acompanhe de forma interativa a Programação Científica do Congresso

\section{ATRAVÉS DO APLICATIVO OS PARTICIPANTES} PODERÃO:

- Adicionar atividades de maior interesse em sua agenda de favoritos

- Fazer o download dos slides que forem disponibilizados pelos palestrantes

- Avaliar as atividades através da classificação por estrelas 


\section{Rio de Janeiro}

\section{"Cidade maravillhosa, \\ cheia de encantos mil|!"}

\section{Rio de Janeiro, berço do samba e da bossa nova, com praias deslumbrantes e montanhas desafiadoras, cultura e arte. Éuma mistura de ritmos, cores e alegria.}

Capital do estado homônimo, no sudeste do Brasil, a cidade do Rio de Janeiro - ou, simplesmente, Rio para os mais íntimos - é o destino brasileiro mais conhecido no exterior e um dos mais visitados na América Latina e no Hemisfério Sul. Em julho de 2012, parte de seu território foi considerado Patrimônio Cultural da Humanidade pela UNESCO com o título de "Rio de Janeiro: Paisagem Carioca entre Montanha e Mar".

É o espelho do Brasil para o resto do mundo, seja positiva ou negativamente, o epíteto de "cidade maravilhosa", cantado na marchinha composta por André Filho e arranjada por Silva Sobreira para o Carnaval de 1935 e título deste texto é o hino mais famoso entoado para glorificar a cidade, cartão-postal do Brasil.

É a segunda maior metrópole brasileira, atrás somente de São Paulo e, apesar de todo um processo de urbanização não planejado em sua história, a cidade maravilhosa faz jus ao seu epíteto e no que diz respeito à hospitalidade e turismo agrada a todos os tipos de turistas. Aliás, parte considerável da economia atual do Rio é voltada para o turismo. Do Pão de Açúcar ao Cristo Redentor, passando pelas praias da Zona Sul e da Barra da Tijuca, a cidade oferece um catálogo amplo de patrimônio arquitetônico, efervescência cultural e paisagens naturais. O prédio do Biblioteca Nacional, o Palácio da Guanabara, os famosos Arcos da Lapa, a suntuosidade da Pedra da Gávea e a pompa do Theatro Municipal completam o roteiro de que não procura só carnaval, samba e bossa nova. Sede das Olímpiadas em 2016, a cidade ganhou ainda mais destaque internacional, revitalizando pontos antes esquecidos pelas autoridades, como o Estádio do Maracanã, que foi testemunha da dolorosa derrota da seleção brasileira sobre o Uruguai na Copa do Mundo de 1950. O estádio, no entanto, parece não ser mesmo o melhor amuleto de sorte do Brasil, já que, mais uma vez, ao sediar uma final de Copa de Mundo, em 2014, testemunho o terrível 7 a 1 da Alemanha sobre o Brasil.

Outro local revitalizado para os eventos olímpicos foi o porto, hoje mais conhecido como "Porto Maravilha". O objetivo dessa obra era revitalizar a área ocupada pelo Porto além de criar uma nova dinâmica no centro da cidade. Construção de ciclofaixas, corredores de ônibus e do primeiro sistema de Veículo Leve sobre Trilhos - VLT para os cariocas - complementaram o projeto. O plano parece ter dado certo, uma vez que um novo espaço cultural, concentrado sobretudo na figura do Museu do Amanhã, levou o turista e o carioca à frequentar o centro novamente.

Imortalizada nas composições de Vinicius de Moraes e Tom Jobim, a bossa nova continua entoando hinos de amor e de beleza ao Rio. Isso, sem contar, a literatura de Manuel Bandeira e tantos outros escritores brasileiros. O barquinho e o violão cantados pela voz doce de Nara Leão e os "olhos de cigana oblíqua e dissimulada" da personagem Capitu de Machado de Assis continuam sendo orgulho e patrimônio cultural dos cariocas e dos brasileiros. E, recentemente, mais um título veio para comprovar de vez a importância da cidade maravilhosa, o Cristo Redentor passou a ser considerado pela UNESCO como uma das 7 maravilhas do mundo contemporâneo.

Rio, cidade maravilhosa, cartão-postal do Brasil, docemente cantada pela Bossa Nova e pelos maiores sambistas brasileiros, contraste de beleza e pobreza, nos ensina a cada dia o quão maravilhoso e deslumbrante pode ser um pôr do sol desde que estejamos prontos para olhá-lo e valorizar a vida em suas pequenezas. 

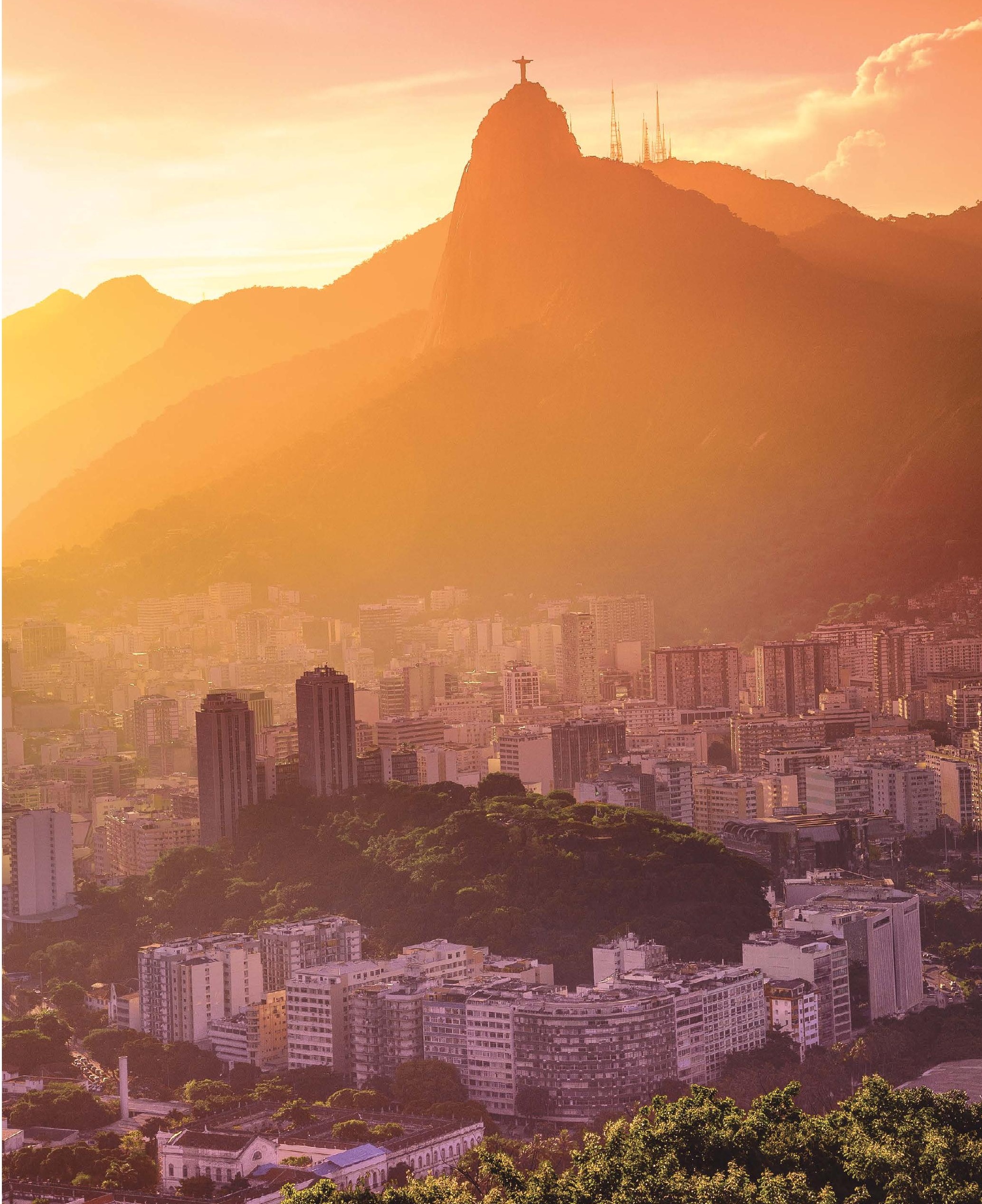


\section{Agenda}

\section{Reuniões Administrativas}

\section{Provas}

\section{Assembleias}

\section{DE ABRIL • QUINTA-FEIRA}

\begin{tabular}{l|l|l}
\hline $08 \mathrm{~h} / 13 \mathrm{~h}$ & Auditório 10 & Prova DECA \\
\hline $\mathbf{1 3 h} / 17 \mathrm{~h}$ & Auditório 10 & Reunião DECA \\
\hline $09 \mathrm{~h} / 10 \mathrm{~h}$ & Sala de Reuniões Administrativas & Reunião Cooperativa RS \\
\hline $10 \mathrm{~h} / 12 \mathrm{~h}$ & Sala de Reuniões Administrativas & Diretoria SBCCV e Diretoria SBCEC \\
\hline $12 \mathrm{~h}$ & Auditório 1 & Reunião DECEM \\
\hline $12 \mathrm{~h} / 14 \mathrm{~h}$ & Sala de Reuniões Administrativas & Reunião ACCERJ
\end{tabular}

\section{DE ABRIL • SEXTA-FEIRA}

\begin{tabular}{|l|l|l}
\hline 09h/10h & Sala de Reuniões Administrativas & Reunião SCICVESP \\
\hline $11 \mathrm{~h} / 12 \mathrm{~h}$ & Sala de Reuniões Administrativas & Reunião SMCCV \\
\hline $12 \mathrm{~h} / 13 \mathrm{~h}$ & Sala de Reuniões Administrativas & Reunião SNNCCV \\
\hline $13 \mathrm{~h} / 14 \mathrm{~h}$ & Sala de Reuniões Administrativas & Reunião BJCVS \\
\hline $14 \mathrm{~h} / 16 \mathrm{~h}$ & Sala de Reuniões Administrativas & Reunião SCOCCV \\
\hline $16 \mathrm{~h} / 18 \mathrm{~h}$ & Sala de Reuniões Administrativas & Reunião DECAM \\
\hline $18 \mathrm{~h} 15$ & Auditório 1 & AGO e AGE SBCCV
\end{tabular}

\section{DE ABRIL • SÁBADO}

\begin{tabular}{l|l|l}
\hline 10h/11h & Sala de Reuniões Administrativas & Assembleia ACCCV \\
\hline $13 \mathrm{~h} / 16 \mathrm{~h}$ & Auditório 10 & Prova SBCEC
\end{tabular}




\section{Atividades Científicas}

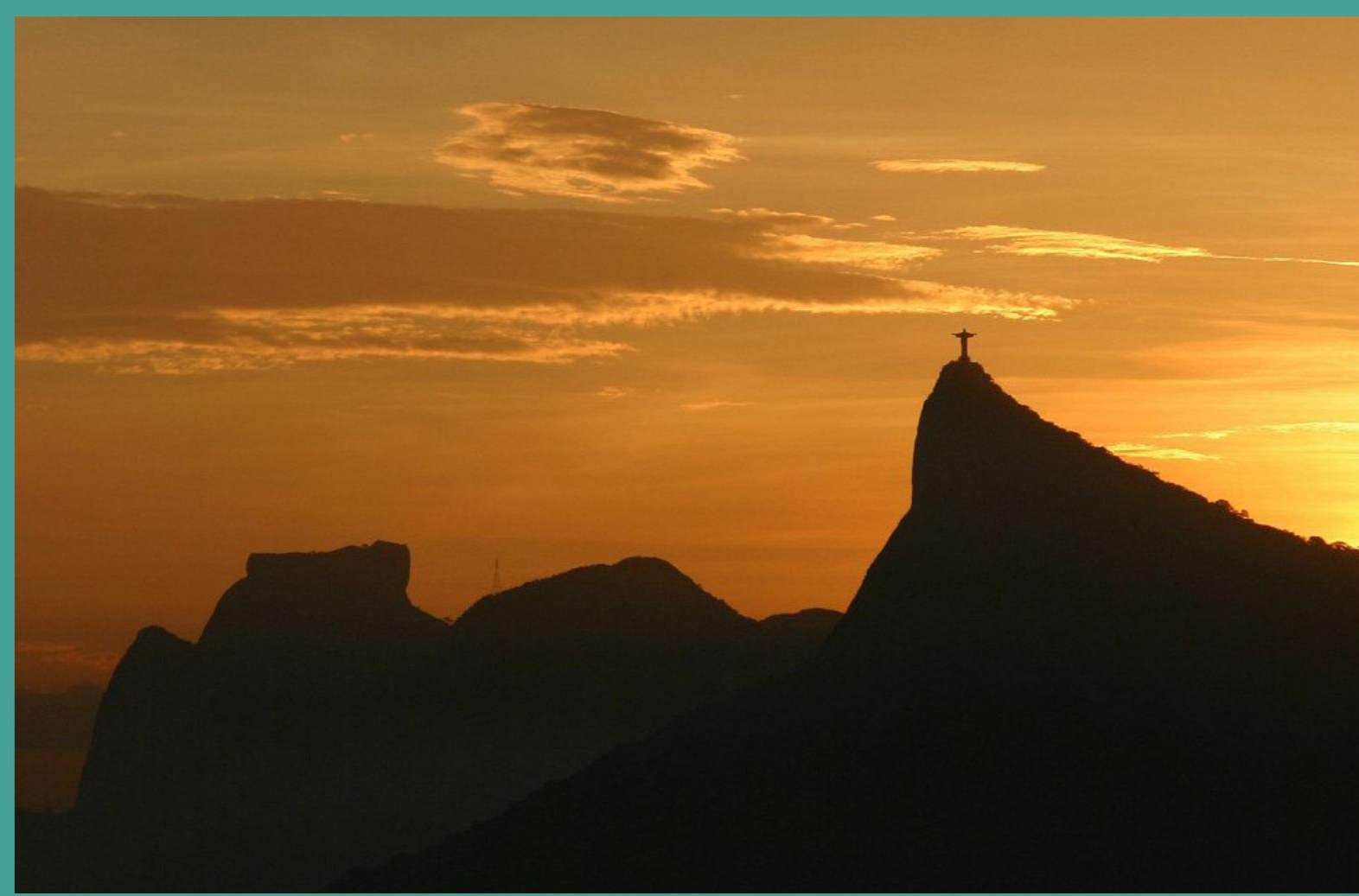

Programa Científico do Congresso 


\section{- AUDITÓRIO 1}

\section{DEPARTAMENTO DE CIRURGIA ENDOVASCULAR E MINIMAMENTE INVASIVA - DECEM}

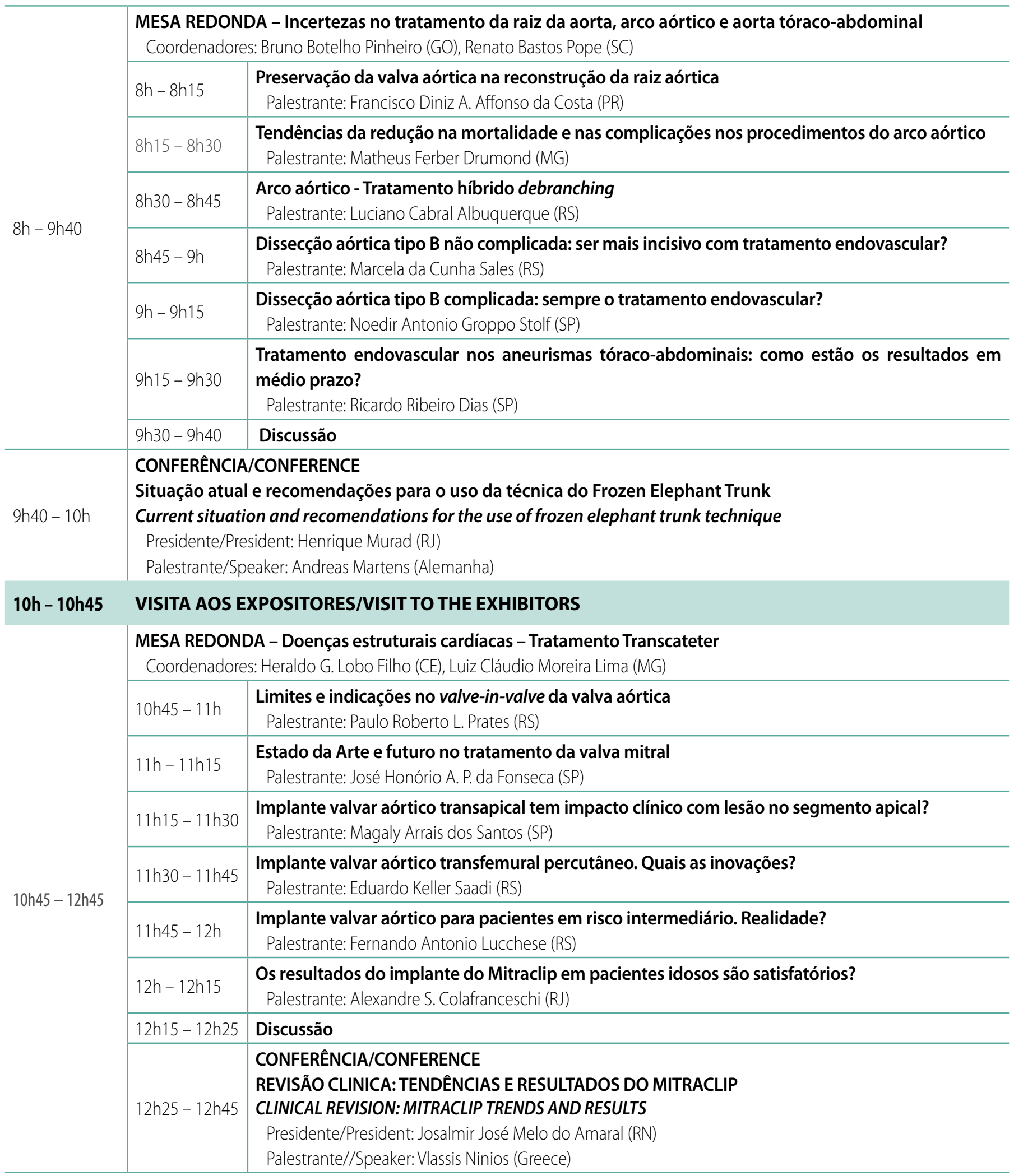




\begin{tabular}{|c|c|c|}
\hline $12 \mathrm{~h} 45-14 \mathrm{~h}$ & \multicolumn{2}{|c|}{ ALMOÇO/SIMPÓSIO SATÉLITE } \\
\hline \multirow{8}{*}{$14 h-15 h 40$} & \multicolumn{2}{|c|}{$\begin{array}{l}\text { MESA REDONDA - Reparo valvar mitral, acesso minimamente invasivo e próteses de liberação rápida } \\
\text { Coordenadores: João Carlos F. Leal (SP), Melchior Luiz Lima (ES) }\end{array}$} \\
\hline & $14 \mathrm{~h}-14 \mathrm{~h} 15$ & $\begin{array}{l}\text { Insuficiência mitral funcional. Trocar ou reparar? } \\
\text { Palestrante: Francisco Gregori Jr (PR) }\end{array}$ \\
\hline & $14 h 15-14 h 30$ & $\begin{array}{l}\text { Minitoracotomia anterior direita para o reparo valvar mitral. Resultados atuais } \\
\text { Palestrante: Wanewman Lins G. de Andrade (BA) }\end{array}$ \\
\hline & $14 h 30-14 h 45$ & $\begin{array}{l}\text { Qual o paciente ideal para troca valvar aórtica pelo acesso minimamente invasivo? } \\
\text { Palestrante: Carlos Manuel de A. Brandão (SP) }\end{array}$ \\
\hline & $14 h 45-15 h 00$ & $\begin{array}{l}\text { Dicas e truques para uma abordagem minimamente invasiva } \\
\text { Palestrante: Olívio Alves de Souza Neto (RJ) }\end{array}$ \\
\hline & $15 h-15 h 15$ & $\begin{array}{l}\text { Desempenho hemodinâmico da prótese de liberação rápida } \\
\text { Palestrante: Gustavo leno Judas (SP) }\end{array}$ \\
\hline & $15 h 15-15 h 30$ & $\begin{array}{l}\text { Robótica no reparo valvar mitral } \\
\text { Palestrante: Robinson Poffo (SP) }\end{array}$ \\
\hline & $15 h 30-15 h 40$ & Discussão \\
\hline $15 h 40-16 h$ & \multicolumn{2}{|c|}{$\begin{array}{l}\text { CONFERÊNCIA } \\
\text { A troca valvar aórtica com bioprótese de liberação rápida e o acesso minimamente invasivo } \\
\text { Aortic valve replacement with rapid deployment bioprothesis and minimally invasive access } \\
\text { Presidente/President: Orlando Petrucci Jr (SP) } \\
\text { Conferencista/Speaker: Andreas Martens (GER) }\end{array}$} \\
\hline
\end{tabular}

16h - $16 \mathrm{~h} 30$ VISITA AOS EXPOSITORES/VISIT TO THE EXHIBITORS

\begin{tabular}{|c|c|c|}
\hline \multirow{3}{*}{ 16h30/18h30 } & 16h30/16h35 & $\begin{array}{l}\text { ABERTURA } \\
\begin{array}{l}\text { Composição da Mesa: Fabio Biscegli Jatene (SP) } \\
\text { Rui M.S. Almeida (PR) } \\
\text { Renato A. K. Kalil (RS) } \\
\text { Eduardo Augusto V. Rocha (MG) } \\
\text { Henrique B. Furtado (TO) } \\
\text { Melchior Luiz LIma (ES) } \\
\text { Carlos Manuel A. Brandão (SP) } \\
\text { Bruno Botelho Pinheiro (GO) } \\
\text { Boas vindas pelo Coordenador do Congresso: Henrique Murad (RJ) } \\
\text { Comissão Local: Andrey José de Oliveira Monteiro (RJ), Marcelo Sávio da S. Martins (RJ), } \\
\text { Mário Ricardo Amar (RJ), Olívio Alves de Souza Neto (RJ) }\end{array}\end{array}$ \\
\hline & $16 h 35 / 17 h$ & Palavra do presidente - Fabio Biscegli Jatene (SP) \\
\hline & 17h/18h30 & $\begin{array}{l}\text { PALESTRAS DE ABERTURA } \\
\text { A hemodinâmica da regurgitação mitral e sua resposta ao reparo ou troca } \\
\text { Understanding hemodynamics of valve regurgitation and its response to repair or replacement } \\
\text { Palestrante/Speaker: Hartzell Schaff (EUA) } \\
\text { Do enxerto de veia safena ao totalmente arterial e mini-invasivo: qual é o padrão de tratamento hoje? } \\
\text { From saphenous vein to total arterial and minimally invasive: What is the standard of care for } \\
\text { CABG today? } \\
\text { Palestrante/Speaker: John D. Puskas (EUA) } \\
\text { Evidências recentes dos resultados da revascularização miocárdia em insuficiência cardíaca/ } \\
\text { Surgical revascularization for heart failure: recent evidences } \\
\text { Palestrante/Speaker: Eric Velasquez (EUA) }\end{array}$ \\
\hline
\end{tabular}




\section{-AUDITÓRI0 2}

\section{DEPARTAMENTO DE CIRURGIA CARDIOVASCULAR PEDIÁTRICA - DCCVPed}

\begin{tabular}{|c|c|c|}
\hline $8 h-8 h 30$ & \multicolumn{2}{|l|}{ ABERTURA } \\
\hline \multirow{5}{*}{$8 h 30-10 h$} & \multicolumn{2}{|c|}{$\begin{array}{l}\text { MESA REDONDA - Cirurgia de Blalock: por que ainda não temos resultados melhores? } \\
\text { Coordenadores: José Teles de Mendonça (SE), Milton Ary Meier (RJ) }\end{array}$} \\
\hline & $8 h 30-8 h 45$ & $\begin{array}{l}\text { Blalock: acesso central ou toracotomia? } \\
\text { Palestrante: Pedro Rafael Salerno (PE) }\end{array}$ \\
\hline & $8 h 45-9 h$ & $\begin{array}{l}\text { Qual o critério para escolha do diâmetro do shunt? } \\
\text { Palestrante: Fernando Antibas Atik (DF) }\end{array}$ \\
\hline & $9 h-09 h 15$ & $\begin{array}{l}\text { Quando não temos outra opção e precisamos fazer o Blalock } \\
\text { Palestrante: Carla Tanamati (SP) }\end{array}$ \\
\hline & $9 h 15-09 h 30$ & $\begin{array}{l}\text { Como evitar as complicações mais comuns (sangramento, trombose, hipóxia, baixo débito } \\
\text { sistêmico) } \\
\text { Palestrante: Beatriz Helena S. Furlanetto (SP) }\end{array}$ \\
\hline \multirow{4}{*}{$10 h 45-12 h 15$} & \multicolumn{2}{|c|}{$\begin{array}{l}\text { MESA REDONDA - Tetralogia de Fallot } \\
\text { Coordenadores: Marcelo M. Cascudo (RN), Valdester C. Pinto Jr. (CE) }\end{array}$} \\
\hline & $10 h 45-11 h$ & $\begin{array}{l}\text { Evolução em longo prazo } \\
\text { Palestrante: Miguel Angel Maluf (SP) }\end{array}$ \\
\hline & $11 h-11 h 15$ & $\begin{array}{l}\text { Quando indicar implante de válvula pulmonar em pacientes submetidos à correção de } \\
\text { Tetralogia de Fallot } \\
\text { Palestrante: Fábio Binhara Navarro (PR) }\end{array}$ \\
\hline & $11 \mathrm{~h} 15-11 \mathrm{~h} 30$ & $\begin{array}{l}\text { Qual o melhor substituto valvar em posição pulmonar? } \\
\text { Palestrante: Valdester C. Pinto Jr (CE) }\end{array}$ \\
\hline $12 \mathrm{~h} 15-14 \mathrm{~h}$ & \multicolumn{2}{|c|}{ SIMPÓSIO SATÉLITE LIVANOVA } \\
\hline \multirow{3}{*}{$14 h-15 h$} & \multicolumn{2}{|c|}{$\begin{array}{l}\text { SESSÃO DE TEMAS LIVRES I } \\
\text { Coordenadores: Beatriz Helena S. Furlanetto (SP), Wilson Luiz da Silveira (GO) }\end{array}$} \\
\hline & $14 h-14 h 15$ & $\begin{array}{l}\text { TL01 - Re-Intervenções durante o curso de ECMO em pacientes pediátricos e portadores de } \\
\text { cardiopatias congênitas } \\
\text { Autor: Leonardo Augusto Miana (SP) } \\
\text { Comentador: Fernando Antoniali (SP) }\end{array}$ \\
\hline & $14 h 15-14 h 30$ & $\begin{array}{l}\text { TL02 - O uso da mini-CEC em reoperações de Tetralogia de Fallot minimiza a perda sanguínea } \\
\text { quando comparada com o circuito de CEC convencional } \\
\text { Autor: Carla Tanamati (SP) } \\
\text { Comentador: Miguel Angel Maluf (SP) }\end{array}$ \\
\hline
\end{tabular}




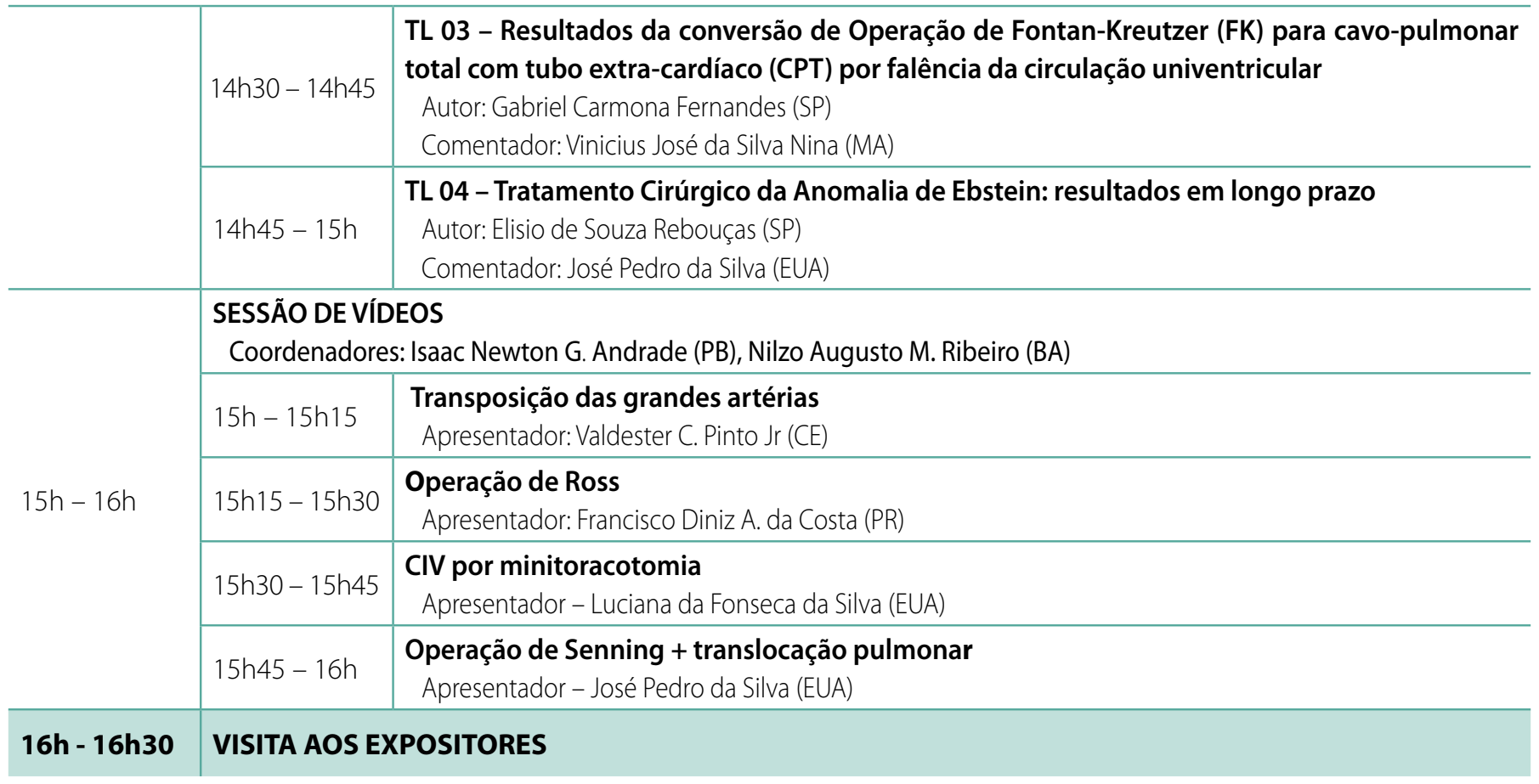

\section{- AUDITÓRIO 3}

\section{DEPARTAMENTO DE CIRCULAÇÃO EXTRACORPÓREA E ASSISTÊNCIA CIRCULATÓRIA MECÂNICA - DECAM}

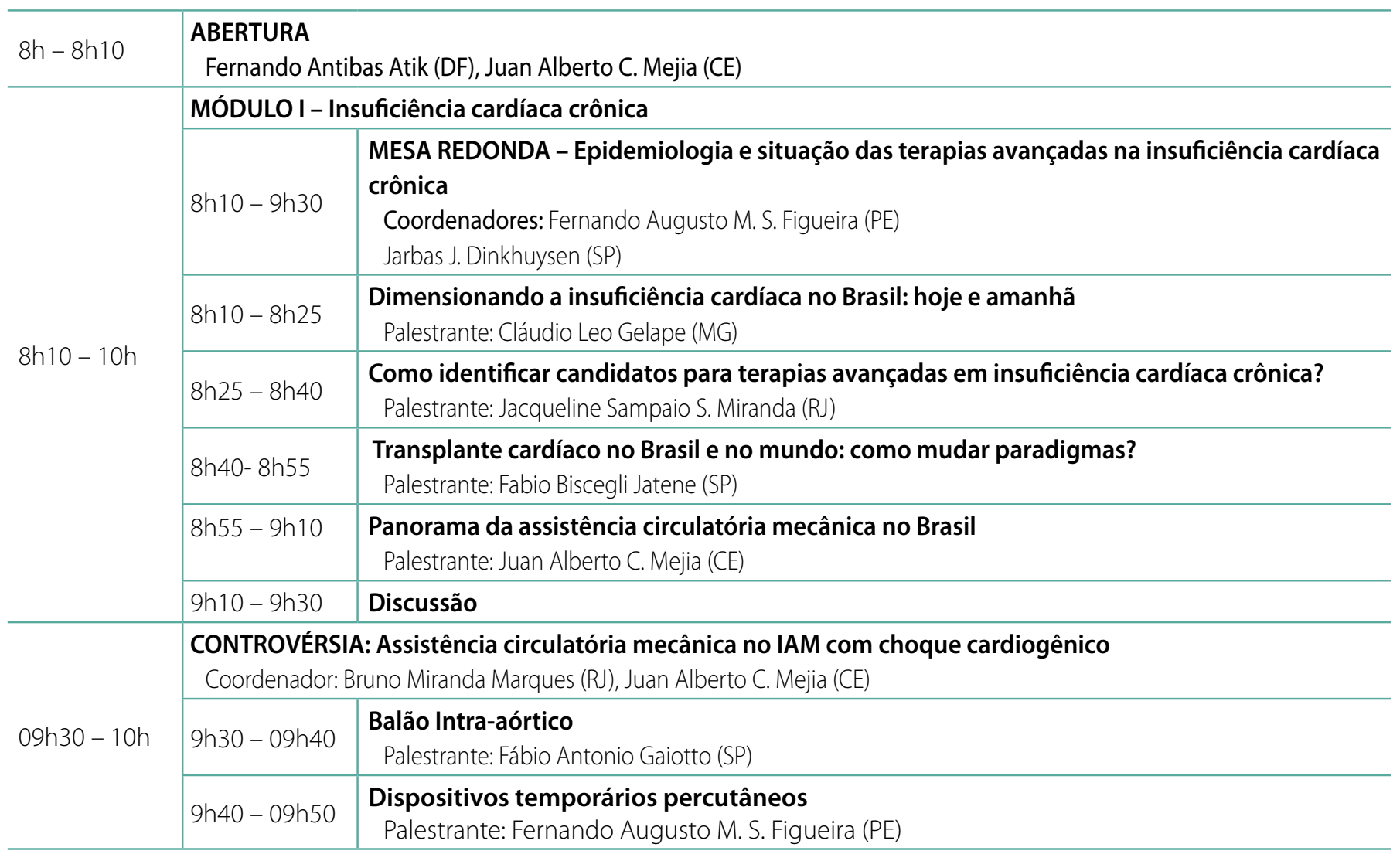




\begin{tabular}{|c|c|c|}
\hline & $9 h 50-9 h 55$ & $\begin{array}{l}\text { Réplica } \\
\text { Palestrante: Fábio Antonio Gaiotto (SP) }\end{array}$ \\
\hline & $9 h 55-10 h$ & $\begin{array}{l}\text { Tréplica } \\
\text { Palestrante: Fernando Augusto M. S. Figueira (PE) }\end{array}$ \\
\hline 10h - $10 h 45$ & \multicolumn{2}{|c|}{ VISITA AOS EXPOSITORES } \\
\hline \multirow{8}{*}{$10 h 45-12 h 15$} & \multicolumn{2}{|c|}{ MÓDULO II - Desafios da assistência circulatória mecânica } \\
\hline & $10 h 45-11 \mathrm{~h} 05$ & $\begin{array}{l}\text { CONFERÊNCIA - Instituições de alta confiabilidade e a geração de valor ao cuidado de } \\
\text { pacientes com insuficiência cardíaca avançada } \\
\text { Presidente: Paulo Manuel Pêgo-Fernandes (SP) } \\
\text { Conferencista: Alexandre S. Colafranceschi (RJ) }\end{array}$ \\
\hline & $11 \mathrm{~h} 05-12 \mathrm{~h} 15$ & $\begin{array}{l}\text { MESA REDONDA - Insuficiência cardíaca aguda } \\
\text { Coordenadores: Luiz Fernando Kubrusly (PR), Paulo Manuel Pêgo-Fernandes (SP) }\end{array}$ \\
\hline & $11 \mathrm{~h} 05-11 \mathrm{~h} 20$ & $\begin{array}{l}\text { Epidemiologia e fisiopatologia do choque cardiogênico } \\
\text { Palestrante: Jacqueline Sampaio S. Miranda (RJ) }\end{array}$ \\
\hline & $11 \mathrm{~h} 20-11 \mathrm{~h} 35$ & $\begin{array}{l}\text { É possível estabelecer um limite farmacológico para o resgate de órgãos na insuficiên- } \\
\text { cia cardíaca aguda? } \\
\text { Palestrante: Marcelo W. Montera (RJ) }\end{array}$ \\
\hline & $11 \mathrm{~h} 35-11 \mathrm{~h} 50$ & $\begin{array}{l}\text { Qual o dispositivo de assistência circulatória mecânica ideal na insuficiência cardíaca aguda? } \\
\text { Palestrante: Fernando Augusto M. S. Figueira (PE) }\end{array}$ \\
\hline & $11 \mathrm{~h} 50-12 \mathrm{~h} 05$ & $\begin{array}{l}\text { INTERMACS 0: quando jogar a toalha? } \\
\text { Palestrante: Fernando Antibas Atik (DF) }\end{array}$ \\
\hline & $12 \mathrm{~h} 05-12 \mathrm{~h} 15$ & Discussão \\
\hline $12 \mathrm{~h} 15-14 \mathrm{~h}$ & \multicolumn{2}{|c|}{ ALMOÇO/SIMPÓSIO SATÉLITE } \\
\hline \multirow{5}{*}{$14 h-16 h$} & \multicolumn{2}{|c|}{$\begin{array}{l}\text { MÓDULO III - Sessão conjunta DECAM/SBCCV e SBCEC: problemas encontrados e soluções na condução } \\
\text { de circulação extracorpórea e ECMO } \\
\text { Coordenadores: Fernando Antibas Atik (DF), Juan Alberto C. Mejia (CE), Sintya Tertuliano Chalegre (PE) }\end{array}$} \\
\hline & $14 h-14 h 30$ & $\begin{array}{l}\text { CASO CLÍNICO } 1 \text { - Dissecção aguda da aorta - estratégias de perfusão e proteção cerebral } \\
\text { Apresentador: Cláudio Ribeiro da Cunha (MG) } \\
\text { Painel de Discussão: Fábio Antonio Gaiotto (SP), Marcelo Ramalho Fernandes (RJ), Noedir A. G. } \\
\text { Stolf (SP), Paulo Roberto L. Prates (RS) }\end{array}$ \\
\hline & $14 h 30-15 h$ & $\begin{array}{l}\text { CASO CLíNICO } 2 \text { - Cirurgia cardiovascular com aorta em porcelana } \\
\text { Apresentador: Fábio Antonio Gaiotto (SP) } \\
\text { Painel de Discussão: Fábio Murilo da Costa (MG) , Jarbas J. Dinkhuysen (SP),Marcelo Ramalho } \\
\text { Fernandes (RJ) , Paulo Manuel Pêgo-Fernandes (SP), Ricardo Ribeiro Dias (SP) }\end{array}$ \\
\hline & $15 h-15 h 30$ & $\begin{array}{l}\text { CASO CLÍNICO } 3 \text { - Hipoxemia durante a ECMO } \\
\text { Apresentador: Fernando Augusto M. S. Figueira (PE) } \\
\text { Painel de Discussão: Alexandre Siciliano Colafranceschi (RJ), Ana Paula Noronha da Silva (SP), } \\
\text { Marcelo W. Montera (RJ), Stêvan Krieger Martins (SP) }\end{array}$ \\
\hline & $15 h 30-16 h$ & $\begin{array}{l}\text { CASO CLÍNICO } 4 \text { - Hipotensão durante a ECMO } \\
\text { Apresentador: Bruno Miranda Marques (RJ) } \\
\text { Painel de Discussão: Juan A. C. Mejia (CE), Fernando Antibas Atik (DF), Fernando Antoniali (SP), } \\
\text { Marcelo W. Montera (RJ), Ana Paula Noronha (SP) }\end{array}$ \\
\hline $16 h-16 h 30$ & \multicolumn{2}{|c|}{ VISITA AOS EXPOSITORES } \\
\hline
\end{tabular}




\section{- AUDITÓRIO 4}

\section{DEPARTAMENTO DE CARDIOLOGIA - DECARDIO}

\begin{tabular}{|c|c|c|}
\hline $8 h-8 h 10$ & \multicolumn{2}{|c|}{$\begin{array}{l}\text { ABERTURA } \\
\text { DECARDIO - Ricardo A. Benfatti (MS) }\end{array}$} \\
\hline \multirow{5}{*}{$08 h 10-09 h 50$} & \multicolumn{2}{|c|}{$\begin{array}{l}\text { MÓDULO I - MESA REDONDA - Atualizações em valvopatias } \\
\text { Presidente: Januário Manuel de Souza (SP) } \\
\text { Moderador: Alexandre C. Hueb (SP) } \\
\text { Debatedores: Fabrício Otávio G. Teixeira(ES), Henrique Barsanulfo Furtado (TO) }\end{array}$} \\
\hline & 08h10-08h30 & $\begin{array}{l}\text { Novidades no diagnóstico por imagem das valvopatias mitral e aórtica } \\
\text { Palestrante/Speaker: Eric Velasquez (USA) } \\
\text { Discussão }\end{array}$ \\
\hline & $8 h 35-08 h 55$ & $\begin{array}{l}\text { Estenose aórtica e TAVI. Resultados atuais } \\
\text { Palestrante: Diego Felipe Gaia dos Santos (SP) } \\
\text { Discussão }\end{array}$ \\
\hline & $9 h 00-09 h 20$ & $\begin{array}{l}\text { Tratamento cirúrgico e intervencionista da insuficiência mitral } \\
\text { Palestrante: Ricardo Adala Benfatti (MS) } \\
\text { Discussão }\end{array}$ \\
\hline & $9 h 25-09 h 45$ & $\begin{array}{l}\text { Cirurgia valvar minimamente invasiva. Onde estamos no Brasil? } \\
\text { Palestrante: Olívio A. de Souza Neto (RJ) } \\
\text { Discussão }\end{array}$ \\
\hline $10 h-10 h 45$ & \multicolumn{2}{|c|}{ VISITA AOS EXPOSITORES } \\
\hline \multirow{6}{*}{$10 h 45-12 h 35$} & \multicolumn{2}{|c|}{$\begin{array}{l}\text { MÓDULO II - MESA REDONDA - Tópicos atuais em cardiologia } \\
\text { Presidente: José Carlos Dorsa V. Pontes (MS) } \\
\text { Moderador: Fernando Oswaldo D. Rangel (RJ) } \\
\text { Debatedores: Ricardo Mourilhe (RJ), Ricardo Adala Benfatti (MS) }\end{array}$} \\
\hline & $10 h 30-10 h 50$ & $\begin{array}{l}\text { Tratamento da lesão de tronco de coronária esquerda após EXCEL e NOBLE } \\
\text { Palestrante: Walter José Gomes (SP) } \\
\text { Discussão }\end{array}$ \\
\hline & $10 h 55-11 \mathrm{~h} 15$ & $\begin{array}{l}\text { Novas Diretrizes (2016) para indicação de assistência circulatória mecânica na insufi- } \\
\text { ciência cardíaca aguda } \\
\text { Palestrante: Alexandre C. Hueb (SP) } \\
\text { Discussão }\end{array}$ \\
\hline & $11 \mathrm{~h} 20-11 \mathrm{~h} 40$ & $\begin{array}{l}\text { Evidências atuais para indicação do tratamento endovascular nas dissecções aórticas Tipo B } \\
\text { Palestrante: Januário Manuel de Souza (S) } \\
\text { Discussão }\end{array}$ \\
\hline & $11 \mathrm{~h} 45-12 \mathrm{~h} 05$ & $\begin{array}{l}\text { Tratamento farmacológico da insuficiência cardíaca. O que temos de novo? } \\
\text { Palestrante: Ricardo Mourilhe (RJ) } \\
\text { Discussão }\end{array}$ \\
\hline & $12 \mathrm{~h} 10-12 \mathrm{~h} 30$ & $\begin{array}{l}\text { O que temos de novo em hipertensão arterial? Diretrizes } 2016 \\
\text { Palestrante: Antoinette de Oliveira Blackman (DF) } \\
\text { Discussão }\end{array}$ \\
\hline $12 h 15-14 h$ & \multicolumn{2}{|c|}{ ALMOÇO/SIMPÓSIO SATÉLITE } \\
\hline
\end{tabular}




\begin{tabular}{|c|c|c|}
\hline \multirow{4}{*}{$14 h-14 h 50$} & \multicolumn{2}{|c|}{$\begin{array}{l}\text { MÓDULO III - MESA REDONDA - Atualizações em Insuficiência coronariana } \\
\text { Presidente: Walter José Gomes (SP) } \\
\text { Moderador: Antoinette de Oliveira Blackman (DF) } \\
\text { Debatedores: Fabrício Otávio G. Teixeira (ES), Luciano Cabral Albuquerque (RS) }\end{array}$} \\
\hline & $14 h-14 h 15$ & $\begin{array}{l}\text { Novos marcadores na Aterosclerose } \\
\text { Palestrante: Roberto Esporcatte (RJ) } \\
\text { Discussão }\end{array}$ \\
\hline & $14 \mathrm{~h} 20-14 \mathrm{~h} 35$ & $\begin{array}{l}\text { Qual o melhor diagnóstico: perfusão ou anatomia? } \\
\text { Palestrante: Gabriel Camargo (RJ) } \\
\text { Discussão }\end{array}$ \\
\hline & $14 h 40-14 h 50$ & $\begin{array}{l}\text { Resultados da cirurgia de revascularização miocárdica e stents coronarianos na } \\
\text { doença crônica } \\
\text { Palestrante: Bruno Botelho Pinheiro (GO) } \\
\text { Discussão }\end{array}$ \\
\hline \multirow{4}{*}{$14 \mathrm{~h} 50-15 \mathrm{~h} 50$} & \multicolumn{2}{|c|}{$\begin{array}{l}\text { MÓDULO IV - MESA REDONDA - Atualizações em Insuficiência Cardíaca } \\
\text { Presidente: Mário Coli Junqueira de Moraes (RJ) } \\
\text { Moderador: José Teles de Mendonça (SE) } \\
\text { Debatedores: José Wanderley Neto (AL), Noedir Antonio G. Stolf (SP) }\end{array}$} \\
\hline & $14 h 50-15 h 05$ & $\begin{array}{l}\text { Fisiopatologia de remodelamento ventricular } \\
\text { Palestrante: Otoni Moreira Gomes (MG) } \\
\text { Discussão }\end{array}$ \\
\hline & $15 h 10-15 h 25$ & $\begin{array}{l}\text { O que temos de novo nos diagnósticos por imagem na insuficiência cardíaca crônica? } \\
\text { Palestrante/Speaker: Eric Velasquez (USA) } \\
\text { Discussão }\end{array}$ \\
\hline & $15 h 30-15 h 50$ & $\begin{array}{l}\text { Tratamento intervencionista e cirúrgico da insuficiência cardíaca crônica } \\
\text { Palestrante: Alfredo I. Fiorelli (SP) } \\
\text { Discussão }\end{array}$ \\
\hline $16 h-16 h 30$ & \multicolumn{2}{|c|}{ VISITA AOS EXPOSITORES } \\
\hline
\end{tabular}

\section{- AUDITÓRIO 5}

\section{DEPARTAMENTO DE CIRURGIA EXPERIMENTAL - DEPEX}

\begin{tabular}{l|l|l}
\hline $8 h$ & \multicolumn{2}{|l}{ ABERTURA } \\
\hline 8h-10h & $\begin{array}{l}\text { MÓDULO I - MESA REDONDA - Tópicos relevantes em cirurgia cardiovascular } \\
\text { Moderadores: Otoni Moreira Gomes (MG), Walter José Gomes (SP) } \\
\text { Debatedores: Luiz Fernando Caneo (SP), Rui M. S. Almeida (PR) }\end{array}$ \\
\hline & $8 \mathrm{~h}-8 \mathrm{~h} 10$ & $\begin{array}{l}\text { Efeito da hipotermia e choque térmico na proteção miocárdica } \\
\text { Palestrante: Melchior Luiz Lima (ES) }\end{array}$ \\
\hline & $8 \mathrm{~h} 15-08 \mathrm{~h} 25$ & $\begin{array}{l}\text { Discinesia miocárdica do estresse da membrana celular } \\
\text { Palestrante: Otoni Moreira Gomes (MG) }\end{array}$ \\
\hline & $8 \mathrm{~h} 45-08 \mathrm{~h} 55$ & $\begin{array}{l}\text { Regulação do tônus vascular } \\
\text { Palestrante: Dalton Valentim Vassallo (ES) }\end{array}$ \\
\hline & $9 \mathrm{~h}-09 \mathrm{~h} 10$ & $\begin{array}{l}\text { Expestrante: Rodrigo M. Milani (PR) } \\
\text { Palestrante: Sérgio Lima de Almeida (SC) }\end{array}$ \\
\hline
\end{tabular}




\begin{tabular}{|c|c|c|}
\hline & 9h15-09h25 & $\begin{array}{l}\text { Imunossupressão no transplantado - estado atual das pesquisas experimentais } \\
\text { Palestrante: Alfredo I. Fiorelli (SP) }\end{array}$ \\
\hline & $9 h 30-9 h 40$ & $\begin{array}{l}\text { Estudos de viabilidade de stents endovasculares com fibras de nanotubos de carbono } \\
\text { contráteis e inteligentes } \\
\text { Palestrante: Fabio Biscegli Jatene (SP) }\end{array}$ \\
\hline & $9 h 45-10 h$ & $\begin{array}{l}\text { MINI-CONFERÊNCIA - Estado Atual das pesquisas para o desenvolvimento da prótese } \\
\text { transcateter Inovare } \\
\text { Presidente: Otoni M. Gomes (MG) } \\
\text { Conferencista: Diego Felipe Gaia dos Santos (SP) }\end{array}$ \\
\hline 10h- $10 \mathrm{~h} 45$ & \multicolumn{2}{|c|}{ VISITA AOS EXPOSITORES } \\
\hline \multirow{7}{*}{$10 h 45-12 h 15$} & \multicolumn{2}{|c|}{$\begin{array}{l}\text { MÓDULO II - MESA REDONDA - Tópicos relevantes em cirurgia cardiovascular } \\
\text { Moderadores: Dalton Valentim Vassallo (ES), Elias Kallás (MG) } \\
\text { Debatedores: Rodrigo Mussi Milani (PR), Eduardo Augusto V. Rocha (MG) }\end{array}$} \\
\hline & 10h45 - 10h55 & $\begin{array}{l}\text { O que aprendemos com as pesquisas no tratamento das dissecções aórticas? } \\
\text { Palestrante: Noedir A. G. Stolf (SP) }\end{array}$ \\
\hline & $11 \mathrm{~h}-11 \mathrm{~h} 10$ & $\begin{array}{l}\text { Aspectos técnicos com dupla troca valvar aórtica e mitral utilizando prótese aórtica de } \\
\text { liberação rápida } \\
\text { Palestrante: Flávio Almeida Rosa (ES) }\end{array}$ \\
\hline & $11 \mathrm{~h} 15-11 \mathrm{~h} 25$ & $\begin{array}{l}\text { Otimização de resultados em cirurgia de revascularização do miocárdio } \\
\text { Palestrante: Bruno Botelho Pinheiro (GO) }\end{array}$ \\
\hline & $11 \mathrm{~h} 30-11 \mathrm{~h} 40$ & $\begin{array}{l}\text { Importância dos escores de risco na produção de pesquisa clínica } \\
\text { Palestrante: Fernando Antonio Lucchese (RS) }\end{array}$ \\
\hline & $11 \mathrm{~h} 45-11 \mathrm{~h} 55$ & $\begin{array}{l}\text { Estado da arte com TAVI } \\
\text { Palestrante: Fernando Antonio Roquette Reis Filho (MG) }\end{array}$ \\
\hline & $12 \mathrm{~h}-12 \mathrm{~h} 10$ & $\begin{array}{l}\text { Pesquisa experimental de bioprótese aórtica de liberação rápida } \\
\text { Palestrante: Walter José Gomes (SP) }\end{array}$ \\
\hline $12 \mathrm{~h} 15-14 h$ & \multicolumn{2}{|c|}{ ALMOÇO/SIMPÓSIO SATÉLITE } \\
\hline \multirow[t]{6}{*}{$14 h-16 h$} & \multicolumn{2}{|c|}{$\begin{array}{l}\text { MÓDULO III - MESA REDONDA - Tópicos relevantes em cirurgia cardiovascular } \\
\text { Moderadores: Flávio Almeida Rosa (ES), José Carlos Dorsa V. Pontes (MS) } \\
\text { Debatedores: Alexandre C. Hueb (SP), Ricardo Adala Benfatti (MS) }\end{array}$} \\
\hline & $14 h-14 h 10$ & $\begin{array}{l}\text { Efeitos das estatinas no estresse oxidativo } \\
\text { Palestrante: José Carlos Dorsa V. Pontes (MS) } \\
\text { Discussão }\end{array}$ \\
\hline & $14 h 15-14 h 25$ & $\begin{array}{l}\text { Resultados tardios da revascularização miocárdica com e sem CEC } \\
\text { Palestrante: Alexandre C. Hueb (SP) } \\
\text { Discussão }\end{array}$ \\
\hline & $14 h 30-14 h 40$ & $\begin{array}{l}\text { O que aprendi com pesquisas clínicas sobre TAVI } \\
\text { Palestrante: João Carlos F. Leal (SP) } \\
\text { Discussão }\end{array}$ \\
\hline & $14 h 45-14 h 55$ & $\begin{array}{l}\text { Avanços na pesquisa no tratamento da insuficiência valvar mitral por cateter } \\
\text { Palestrante: José Honório de A. Palma da Fonseca (SP) } \\
\text { Discussão }\end{array}$ \\
\hline & $15 h 00-15 h 10$ & $\begin{array}{l}\text { ECMO: Estado atual } \\
\text { Palestrante: Luiz Fernando Caneo (SP) } \\
\text { Discussão }\end{array}$ \\
\hline
\end{tabular}




\begin{tabular}{l|l|l}
\hline & $15 h 15-15 h 25$ & $\begin{array}{l}\text { Análise no seguimento tardio entre troca e plastia valvar } \\
\text { Palestrante: Carlos Manuel de A. Brandão (SP) } \\
\text { Discussão }\end{array}$ \\
\hline $15 h 30-15 h 40$ & $\begin{array}{l}\text { Estenose valvar aótica: TAVI ou técnica convencional? } \\
\text { Palestrante: Charles Simão Filho (MG) } \\
\text { Discussão }\end{array}$ \\
\hline $15 h 45-15 h 55$ & $\begin{array}{l}\text { Estado atual das pesquisas com células tronco } \\
\text { Palestrante: Paulo Roberto S. Brofman (PR) } \\
\text { Discussão }\end{array}$ \\
\hline
\end{tabular}

\section{- AUDITÓRI0 6}

\section{ENCONTRO DOS RESIDENTES}

\begin{tabular}{|c|c|c|}
\hline \multirow{5}{*}{$08 \mathrm{~h}-10 \mathrm{~h}$} & \multicolumn{2}{|c|}{$\begin{array}{l}\text { MESA REDONDA - Como fazer? } \\
\text { Coordenador: Dilmar Cardeal da Cunha (RS) }\end{array}$} \\
\hline & $8 h-08 h 30$ & $\begin{array}{l}\text { Revascularização miocárdica } \\
\text { Palestrante: Luís Alberto O. Dallan (SP) }\end{array}$ \\
\hline & $8 \mathrm{~h} 30-9 \mathrm{~h}$ & $\begin{array}{l}\text { Troca de válvula mitral } \\
\text { Palestrante: Magaly Arrais dos Santos (SP) }\end{array}$ \\
\hline & $9 h-09 h 30$ & $\begin{array}{l}\text { Troca de válvula aórtica } \\
\text { Palestrante: Rodrigo Freire Bezerra (SP) }\end{array}$ \\
\hline & $9 h 30-10 h$ & $\begin{array}{l}\text { Interposição de tubo supracoronariano } \\
\text { Palestrante: Francisco Siosney A. Pinto (AL) }\end{array}$ \\
\hline $10 h-10 h 45$ & \multicolumn{2}{|c|}{ VISITA AOS EXPOSITORES } \\
\hline \multirow{4}{*}{$10 h 45-12 h 15$} & \multicolumn{2}{|c|}{$\begin{array}{l}\text { MESA REDONDA - Cirurgia Cardiovascular após a Residência Médica } \\
\text { Coordenador: Rafaela da Hora Sales (AL) }\end{array}$} \\
\hline & $10 h 45-11 \mathrm{~h} 15$ & $\begin{array}{l}\text { Como criar uma equipe de cirurgia cardiovascular no interior do Brasil } \\
\text { Palestrante: Décio Cardoso da Silva Jr. (BA) }\end{array}$ \\
\hline & $11 \mathrm{~h} 15-11 \mathrm{~h} 45$ & $\begin{array}{l}\text { Como negociar salários e honorários } \\
\text { Palestrante: Eliudem Galvão (ES) }\end{array}$ \\
\hline & $11 \mathrm{~h} 45-12 \mathrm{~h} 15$ & $\begin{array}{l}\text { Tudo o que o cirurgião cardiovascular precisa saber sobre empresas } \\
\text { Palestrante: Alexei Ribeiro Nunes (RJ) }\end{array}$ \\
\hline $12 \mathrm{~h} 15-14 \mathrm{~h}$ & \multicolumn{2}{|c|}{ ALMOÇO/SIMPÓSIO SATÉLITE } \\
\hline $14 h-16 h$ & \multicolumn{2}{|c|}{ ASSEMBLEIA ABRECCV e Debate entre as chapas candidatas à Diretoria da ABRECCV } \\
\hline
\end{tabular}

\section{- AUDITÓRI0 7}

\section{HANDS ON SOCIEDADE BRASILEIRA DE CIRCULAÇÃO EXTRACORPÓREA}

\section{CIRCUITOS E MONTAGENS EM ECMO}




\section{- AUDITÓRIO 8}

\section{CURSO DE METODOLOGIA CIENTÍFICA}

Coordenador: Domingo M. Braile (SP)

\begin{tabular}{|c|c|}
\hline $08 h-10 h$ & $\begin{array}{l}\text { Noções básicas de Estatística - Curvas atuariais e Meta análise } \\
\text { Palestrante: Palestrante: Moacir Godoy (SP) }\end{array}$ \\
\hline 10h0- 10h45 & VISITA AOS EXPOSITORES \\
\hline $10 h 45-12 h 30$ & $\begin{array}{l}\text { Metodologia aplicada à concepção e elaboração de um artigo científico } \\
\text { Palestrante: Dorotéia Souza (SP) }\end{array}$ \\
\hline $12 h 30-14 h$ & ALMOÇO/SIMPÓSIO SATÉLITE \\
\hline $14 h-15 h$ & $\begin{array}{l}\text { Pesquisa Bibliográfica } \\
\text { Palestrante: A DEFINIR }\end{array}$ \\
\hline $15 h-16 h$ & $\begin{array}{l}\text { Como ler criticamente um artigo científico } \\
\text { Palestrante: Alfredo José Rodrigues (SP) }\end{array}$ \\
\hline $16 h-16 h 30$ & VISITA AOS EXPOSITORES \\
\hline
\end{tabular}

\section{- AUDITÓRIO 9}

\section{LIGA DOS ACADÊMICOS EM CIRURGIA CARDIOVASCULAR}

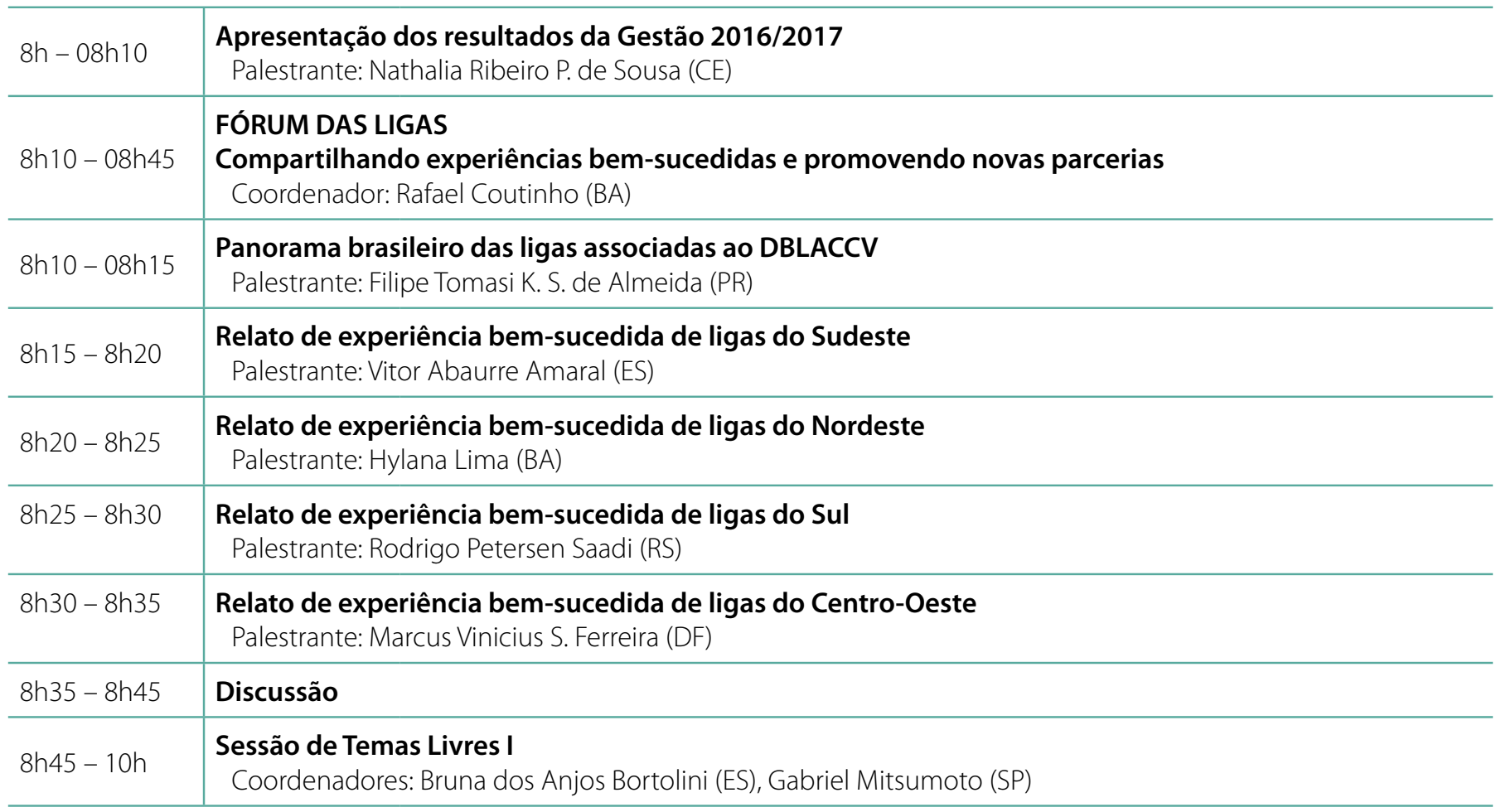




\begin{tabular}{|c|c|c|}
\hline & $8 h 45-9 h$ & $\begin{array}{l}\text { TL 01- Impacto da hipertensão pulmonar pré-operatória nos desfechos de pacientes } \\
\text { submetidos à cirurgia cardiovascular } \\
\text { Autor: Armindo Jreige Jr (DF) } \\
\text { Comentador: Valdo José Carreira (RJ) }\end{array}$ \\
\hline & $9 h 00-09 h 15$ & $\begin{array}{l}\text { TL } 02 \text { - Utilização de bomba centrífuga conectada à cânula implantável de longa du- } \\
\text { ração como suporte circulatório pediátrico: desenvolvendo soluções economicamente } \\
\text { viáveis } \\
\text { Autor: Rafael Ceconi (SP) } \\
\text { Comentador: Luiz Fernando Kubrusly (PR) }\end{array}$ \\
\hline & 9h15-09h30 & $\begin{array}{l}\text { TL } 03 \text { - Impacto do desempenho técnico-cirúrgico nos resultados imediatos da cirurg- } \\
\text { ia cardiovascular pediátrica: uma análise preliminar } \\
\text { Autor: Yarla Alves dos Santos (SP) } \\
\text { Comentador: Leonardo Augusto Miana (SP) }\end{array}$ \\
\hline & $9 h 30-09 h 45$ & $\begin{array}{l}\text { TL } 04 \text { - Vasoplegia no pós operatório imediato de cirurgia de revascularização do } \\
\text { miocárdio: fatores preditivos e evolução } \\
\text { Autor: Gustavo Farias Porciúncula (RS) } \\
\text { Comentador: Paulo Roberto B. Évora (SP) }\end{array}$ \\
\hline & $9 h 45-10 h$ & $\begin{array}{l}\text { TL } 05 \text { - Estudo sobre o coração univentricular e suas opções de tratamento } \\
\text { Autor: Bianca Alves de Miranda (RJ) } \\
\text { Comentador: Leonardo Andrade Mulinari (PR) }\end{array}$ \\
\hline $10 h-10 h 30$ & \multicolumn{2}{|c|}{ VISITA AOS EXPOSITORES } \\
\hline \multirow{5}{*}{$10 h 30-11 h 45$} & \multicolumn{2}{|c|}{$\begin{array}{l}\text { MESA REDONDA } \\
\text { Indo além do centro cirúrgico: os dilemas envolvidos na escolha da cirurgia cardiovascular } \\
\text { Coordenadores: Gustavo leno Judas (SP), Leila Nogueira Barros (SP) }\end{array}$} \\
\hline & $10 h 30-10 h 50$ & $\begin{array}{l}\text { A mulher como cirurgiã cardiovascular: a que passo estamos caminhando? } \\
\text { Palestrante: Magaly Arrais dos Santos (SP) }\end{array}$ \\
\hline & $10 h 50-11 \mathrm{~h} 10$ & $\begin{array}{l}\text { Balança vida profissional } \mathbf{x} \text { vida familiar: qual é a medida certa? } \\
\text { Palestrante: Vinicius José da S. Nina (MA) }\end{array}$ \\
\hline & $11 \mathrm{~h} 10-11 \mathrm{~h} 30$ & $\begin{array}{l}\text { Liderança em cirurgia cardiovascular: qual o caminho a se percorrer? } \\
\text { Palestrante: Fabio Biscegli Jatene (SP) }\end{array}$ \\
\hline & $11 \mathrm{~h} 30-11 \mathrm{~h} 45$ & Discussão \\
\hline $11 \mathrm{~h} 45-12 \mathrm{~h} 15$ & \multicolumn{2}{|c|}{$\begin{array}{l}\text { CONFERÊNCIA } \\
\text { Planejamento de carreira: produzindo as ferramentas necessárias para um futuro bem-sucedido } \\
\text { Presidente: Letícia Oliveira (SP) } \\
\text { Conferencista: Rui M. S. Almeida (PR) }\end{array}$} \\
\hline $12 \mathrm{~h} 15-14 \mathrm{~h}$ & \multicolumn{2}{|c|}{ ALMOÇO/SIMPÓSIO SATÉLITE } \\
\hline $14 h-14 h 30$ & \multicolumn{2}{|c|}{$\begin{array}{l}\text { SESSÃO INTEGRATIVA } \\
\text { Conheça um cirurgião cardiovascular de sucesso } \\
\text { Presidente: Nathália Ribeiro P. de Sousa (CE) }\end{array}$} \\
\hline & \multicolumn{2}{|c|}{$\begin{array}{l}\text { MESA 1: Pediátrico } \\
\text { Cirurgião: Fernando R. Moraes Neto (PE) } \\
\text { Facilitador: Rafael Coutinho (BA) }\end{array}$} \\
\hline & \multicolumn{2}{|c|}{$\begin{array}{l}\text { MESA 2: Adulto } \\
\text { Cirurgião: Bayard Gontijo Filho (MG) } \\
\text { Facilitador: Vitor Abaurre Amaral (ES) }\end{array}$} \\
\hline
\end{tabular}




\section{PROGRAMA CIENTíFICO QUUNTA-FERA}

\section{MESA 3: Endovascular}

Cirurgião: Eduardo Keller Saadi (RS)

Facilitador: Marcus Vinicius Silva Ferreira (DF)

MESA 4: Assistência Circulatória e Transplante Cardíaco

Cirurgião: Fernando Augusto M. S. Figueira (CE)

Facilitador: Leila Nogueira Barros (SP)

\section{Sessão de Temas Livres II}

Coordenadores: Armindo Jreige Jr. (DF), Natália Scardua Mariano Alves (ES)

\begin{tabular}{|c|c|c|}
\hline \multirow{3}{*}{$14 h 30-15 h 15$} & $14 \mathrm{~h} 30-14 \mathrm{~h} 45$ & $\begin{array}{l}\text { TL } 06 \text { - Quais as variáveis preditoras de mortalidade em reoperações valvares mitrais? } \\
20 \text { anos de experiência de um único cirurgião } \\
\text { Autor: Daniel Tomasi K. S. de Almeida (PR) } \\
\text { Comentador: Marcos Antonio Cantero (MS) }\end{array}$ \\
\hline & $14 h 45-15 h 00$ & $\begin{array}{l}\text { TL } 07 \text { - Endocardite infecciosa em cirurgias valvares - Avaliação dos critérios ecocar- } \\
\text { diográficos e clínicos como preditores de mortalidade em uma série de casos } \\
\text { Autor: Igor Arantes de Oliveira Góes (SP) } \\
\text { Comentador: Ricardo Nilsson Sgarbieri (SP) }\end{array}$ \\
\hline & $15 h 00-15 h 15$ & $\begin{array}{l}\text { TL } 08 \text { - Influência da impressão 3D na cirurgia cardiovascular } \\
\text { Autor: Thais Lemos de Souza Macedo (RJ) } \\
\text { Comentador: Walter José Gomes (SP) }\end{array}$ \\
\hline $15 h 15-15 h 45$ & \multicolumn{2}{|c|}{$\begin{array}{l}\text { CONFERÊNCIA } \\
\text { Formado em meio a mudanças: a residência em cirurgia cardiovascular que acompanha as novas } \\
\text { nuances da especialidade } \\
\text { Presidente: Filipe Tomasi K. S. de Almeida (PR) } \\
\text { Conferencista: Henrique Murad (RJ) }\end{array}$} \\
\hline $15 h 45-16 h$ & \multicolumn{2}{|c|}{ Assembleia Geral DBLACCV e eleição da Diretoria 2017/2018 } \\
\hline $16 h-16 h 3$ & \multicolumn{2}{|c|}{ VISITA AOS EXPOSITORES } \\
\hline
\end{tabular}




\section{- AUDITÓRIO 1}

\section{SESSÃO ESPECIAL DE TEMAS LIVRES}

\begin{tabular}{|c|c|c|}
\hline \multirow{9}{*}{$8 h-10 h$} & \multicolumn{2}{|c|}{$\begin{array}{l}\text { SESSÃO DE TEMAS LIVRES I } \\
\text { Apresentação e julgamento de temas livres selecionados e candidatos à premiação } \\
\text { Coordenadores: Domingo M. Braile (SP), Henrique Murad (RJ) } \\
\text { Secretário: Débora Klein Ferreira (RS) } \\
\text { Comissão Julgadora: Andrea Dumsch de Aragon Ferreira (PR), Bruno Botelho Pinheiro (GO), Fernando Antibas } \\
\text { Atik (DF), Luciano Cabral Albuquerque (RS), Luiz Cesar Guarita de Souza (PR), Marcelo Matos Cascudo (RN) }\end{array}$} \\
\hline & $8 h 00-08 h 15$ & $\begin{array}{l}\text { TL } 01 \text { - Resultados tardios da reconstrução da via de saída do ventrículo direito com } \\
\text { homoenxertos descelularizados em crianças menores de } 12 \text { anos } \\
\text { Autor: Francisco Diniz A. da Costa (PR) } \\
\text { Comentador: Carla Tanamati (SP) }\end{array}$ \\
\hline & $8 h 15-08 h 30$ & $\begin{array}{l}\text { TL } 02 \text { - Transplante cardíaco em cardiopatias congênitas: resultados e desafios } \\
\text { Autor: Guilherme Viotto Rodrigues da Silva (SP) } \\
\text { Comentador: Valdester Cavalcante Pinto Jr (CE) }\end{array}$ \\
\hline & $8 h 30-08 h 45$ & $\begin{array}{l}\text { TL } 03 \text { - Avaliação do pós-condicionamento isquêmico e da atorvastatina na } \\
\text { prevenção das lesões de reperfusão. Estudo experimental em ratos } \\
\text { Autor: José Carlos Dorsa V. Pontes (MS) } \\
\text { Comentador: João Carlos Jazbik (RJ) }\end{array}$ \\
\hline & $8 h 45-9 h$ & $\begin{array}{l}\text { TL } 04 \text { - A plastia tricúspide associada à intervenção em valva mitral tem impacto? } \\
\text { Autor: Vinicius Fleiria Pimenta (SP) } \\
\text { Comentador: Pablo Maria A. Pomerantzeff (SP) }\end{array}$ \\
\hline & $9 h-09 h 15$ & $\begin{array}{l}\text { TL } 05 \text { - Trombocitopenia após valve-in-valve transcateter } \\
\text { Autor: Renato César de Souza (SP) } \\
\text { Comentador: Cláudio Leo Gelape (MG) }\end{array}$ \\
\hline & $9 h 15-9 h 30$ & $\begin{array}{l}\text { TL } 06 \text { - Tratamento cirúrgico da endocardite infecciosa ativa: preditores de } \\
\text { mortalidade em } 10 \text { anos de seguimento } \\
\text { Autor: Jenny Lourdes Rivas de Oliveira (SP) } \\
\text { Comentador: Rubens Giambroni Filho (RJ) }\end{array}$ \\
\hline & $9 h 30-9 h 45$ & $\begin{array}{l}\text { TL } 07 \text { - Valve-in-valve mitral sequencial (valve-in-valve-in-valve): o fim de reoperações? } \\
\text { Expansão dos limites terapêuticos através de testes hidrodinâmicos in vitro } \\
\text { Autor: Caio César Cardoso (SP) } \\
\text { Comentador: Paulo Roberto L. Prates (RS) }\end{array}$ \\
\hline & $9 h 45-10 h$ & $\begin{array}{l}\text { TL } 08 \text { - Reconstrução da raiz da aorta: existe espaço para outra cirurgia além da } \\
\text { Operação de Tirone? } \\
\text { Autor: Fabrício José de Souza Dinato (SP) } \\
\text { Comentador: Sérgio Caporali de Oliveira (MG) }\end{array}$ \\
\hline $10 h-10 h 45$ & \multicolumn{2}{|c|}{ VISITA AOS EXPOSITORES E SESSÕES DE APRESENTAÇÃO DE PÔSTERES } \\
\hline $10 \mathrm{~h} 45-12 \mathrm{~h} 45$ & \multicolumn{2}{|c|}{$\begin{array}{l}\text { PALESTRAS } \\
\text { Atualidades em Cirurgia Cardiovascular } \\
\text { Coordenadores/Coordinators: Fabio Biscegli Jatene (SP), Paulo Roberto B. Évora (SP), Renato A. K. Kalil (RS) } \\
\text { Secretário/Secretary: Diego Gamarra Moreira (SP) }\end{array}$} \\
\hline & $10 h 45-11 \mathrm{~h} 15$ & $\begin{array}{l}\text { Cirurgia do tromboembolismo pulmonar crônico: indicação, técnicas e resultados } \\
\text { Surgery for chronic pulmonary thromboembolism: indications, techniques and results } \\
\text { Palestrante/Speaker: Michael Madani (USA) }\end{array}$ \\
\hline
\end{tabular}




\begin{tabular}{|c|c|c|}
\hline & $11 \mathrm{~h} 15-11 \mathrm{~h} 45$ & $\begin{array}{l}\text { Novos desenvolvimentos no manejo cirúrgico de cardiomiopatia hipertrófica } \\
\text { New developments in surgical management of hypertrophic cardiomyopathy } \\
\text { Palestrante/Speaker: Hartzell Schaff (USA) }\end{array}$ \\
\hline & $11 \mathrm{~h} 45-12 \mathrm{~h} 15$ & $\begin{array}{l}\text { Mudança de paradigmas na escolha da prótese valvar - dispositivos e abordagens } \\
\text { Changing paradigms in choice of valve prosthesis - devices \& approaches } \\
\text { Palestrante/Speaker: John Puskas (USA) }\end{array}$ \\
\hline & $12 \mathrm{~h} 15-12 \mathrm{~h} 45$ & Discussão/Discussion \\
\hline $12 \mathrm{~h} 45-14 \mathrm{~h}$ & \multicolumn{2}{|c|}{ ALMOÇO/ SIMPÓSIOS } \\
\hline \multirow{7}{*}{$14 h-16 h$} & \multicolumn{2}{|c|}{$\begin{array}{l}\text { MESA REDONDA } \\
\text { Condutas terapêuticas na insuficiência cardíaca de causa isquêmica } \\
\text { Herapeutic conducts in heart failure of ischemic Cause } \\
\text { Coordenadores/Coordinators: Luiz Fernando Kubrusly (PR), Carlos Manuel de A. Brandão (SP) } \\
\text { Secretário/Secretary: Dilmar Cardeal da Cunha (RS) }\end{array}$} \\
\hline & $14 \mathrm{~h} 00-14 \mathrm{~h} 20$ & $\begin{array}{l}\text { Indicações para tratamento clínico ou cirúrgico } \\
\text { Patient selection for medical or surgical management } \\
\text { Palestrante/Speaker: Mauro Paes Leme de Sá (RJ) }\end{array}$ \\
\hline & $14 \mathrm{~h} 20-14 \mathrm{~h} 40$ & $\begin{array}{l}\text { O papel atual da intervenção percutânea } \\
\text { The actual role of percutaneous intervention } \\
\text { Palestrante/Speaker: Luiz Antonio de Carvalho (RJ) }\end{array}$ \\
\hline & $14 h 40-15 h$ & $\begin{array}{l}\text { Técnicas atuais de RM em pacientes com disfunção ventricular } \\
\text { Current surgical approach for CABG in the presence of ventricular disfunction } \\
\text { Palestrante/Speaker: John Puskas (USA) }\end{array}$ \\
\hline & $15 h-15 h 20$ & $\begin{array}{l}\text { Reconstrução ventricular: conceitos atuais e resultados tardios } \\
\text { Surgical ventricular reconstruction: current concepts and late results } \\
\text { Palestrante/Speaker: Eric Velasquez (USA) }\end{array}$ \\
\hline & $15 h 20-15 h 40$ & $\begin{array}{l}\text { O papel dos dispositivos de assistência circulatória e do transplante cardíaco na } \\
\text { insuficiência cardíaca isquêmica avançada } \\
\text { The role of circulatory assistance and transplantation in advanced ischemic cardiomyopathy } \\
\text { Palestrante/Speaker: Alexandre Siciliano Colafranceschi (RJ) }\end{array}$ \\
\hline & $15 h 40-16 h$ & Discussão/Discussion \\
\hline $16 h-16 h 45$ & \multicolumn{2}{|c|}{ VISITA AOS EXPOSITORES E SESSÕES DE APRESENTAÇÃO DE PÔSTERES } \\
\hline \multirow[t]{5}{*}{$16 h 45-18 h 15$} & \multicolumn{2}{|c|}{$\begin{array}{l}\text { ESTUDOS CLíNICOS QUE IMPACTAM NA PRÁTICA CIRÚRGICA } \\
\text { Coordenadores/Coordinators: Luciano Cabral Albuquerque (RS), Renato A. K. Kalil (RS) } \\
\text { Secretário/Secretary: Douglas Barbosa F. Souza (MG) } \\
\text { Comentadores/Commentators: Hartzell Schaff (EUA), John Puskas (EUA) }\end{array}$} \\
\hline & $16 h 45-17 h$ & $\begin{array}{l}\text { EXCEL e NOBLE: lesão de tronco de coronária esquerda } \\
\text { EXCEL and NOBLE: left main coronary artery disease } \\
\text { Palestrante/Speaker: Rodrigo Coelho Segalote (RJ) }\end{array}$ \\
\hline & $17 \mathrm{~h}-17 \mathrm{~h} 15$ & $\begin{array}{l}\text { BEST: multivasculares } \\
\text { BEST: } \text { multivessel disease } \\
\text { Palestrante/Speaker: Orlando C. B. Wender (RS) }\end{array}$ \\
\hline & $17 h 15-17 h 30$ & $\begin{array}{l}\text { SINTAX: multivasculares e tronco coronária esquerda } \\
\text { SINTAX: multivessel and left main coronary artery disease } \\
\text { Palestrante/Speaker: Orlando Petrucci Jr (SP) }\end{array}$ \\
\hline & $17 h 30-17 h 45$ & $\begin{array}{l}\text { MASS: artéria descendente anterior e angina estável } \\
\text { MASS: left anterior descending coronary artery disease and stable angina } \\
\text { Palestrante/Speaker: Alexandre C. Hueb (SP) }\end{array}$ \\
\hline
\end{tabular}




\begin{tabular}{l|l|l}
\hline & $17 \mathrm{~h} 45-18 \mathrm{~h}$ & $\begin{array}{l}\text { ART: uma ou duas mamárias } \\
\text { ART: one or two mammary artery grafts } \\
\text { Palestrante/Speaker: Luís Alberto Oliveira Dallan (SP) }\end{array}$ \\
\hline \multirow{2}{*}{$18 \mathrm{~h}-18 \mathrm{~h} 15$} & $\begin{array}{l}\text { BYPASS REGISTRY: Registro Brasileiro de Cirurgia Cardiovascular } \\
\text { BYPASS: Brazilian Registry of Cardiovascular Surgery } \\
\text { Palestrante/Speaker: Walter José Gomes (SP) }\end{array}$ \\
\cline { 2 - 3 } & \multicolumn{1}{|c|}{ Dicussão/Discussion } \\
\hline
\end{tabular}

\section{- AUDITÓRIO 2}

\section{DEPARTAMENTO DE CIRURGIA CARDIOVASCULAR PEDIÁTRICA - DCCVPed}

\begin{tabular}{|c|c|c|}
\hline \multirow{6}{*}{$8 h 30-10 h$} & \multicolumn{2}{|c|}{$\begin{array}{l}\text { MESA REDONDA } \\
\text { Anomalia de Ebstein } \\
\text { Ebstein Anomaly } \\
\text { Coordenadores/Coordinators: Hartzell V. Schaff (USA) } \\
\text { Luciana Fonseca da Silva (USA) }\end{array}$} \\
\hline & $8 h 30-8 h 45$ & $\begin{array}{l}\text { Avaliação clínica e ecocardiográfica na decisão terapêutica } \\
\text { Clinical and echocardiographic evaluation in the therapeutic decision making } \\
\text { Palestrante/Speaker: Sandra de Jesus Pereira (RJ) }\end{array}$ \\
\hline & $8 h 45-9 h$ & $\begin{array}{l}\text { Arritmias cardíacas na Anomalia de Ebstein: quando e como tratar } \\
\text { Cardiac arrhythmias in Ebstein's anomaly: when and how to treat it } \\
\text { Palestrante/Speaker: Leonardo Andrade Mulinari (PR) }\end{array}$ \\
\hline & $9 h-9 h 15$ & $\begin{array}{l}\text { Opções terapêuticas na atualidade } \\
\text { Current therapeutic options } \\
\text { Palestrante/Speaker: Fernando Antoniali (SP) }\end{array}$ \\
\hline & $9 h 15-9 h 30$ & $\begin{array}{l}\text { Cirurgia de Cone: a evolução da técnica e resultados } \\
\text { Cone reconstruction: evolution and results } \\
\text { Palestrante/Speaker: José Pedro da Silva (USA) }\end{array}$ \\
\hline & $9 h 30-10 h$ & Discussão/Discussion \\
\hline $10 h-10 h 45$ & \multicolumn{2}{|c|}{ VISITA AOS EXPOSITORES E SESSÕES DE APRESENTAÇÃO DE PÔSTERES } \\
\hline \multirow[t]{4}{*}{$10 h 45-12 h 15$} & \multicolumn{2}{|c|}{$\begin{array}{l}\text { MESA REDONDA - Ventrículo único } \\
\text { Coordenadores: Mariano Brasil Terrazas (AM) } \\
\text { Wilson Luiz da Silveira (GO) }\end{array}$} \\
\hline & $10 h 45-11$ h00 & $\begin{array}{l}\text { Devemos manter o fluxo pulmonar anterógrado nos pacientes submetidos à Cirurgia } \\
\text { de Glenn? } \\
\text { Palestrante: Renato Samy Assad (SP) }\end{array}$ \\
\hline & $11 \mathrm{~h}-11 \mathrm{~h} 15$ & $\begin{array}{l}\text { Cirurgia de Fontan com e sem fenestração } \\
\text { Palestrante: Marcello Gomide C. de Faria (RJ) }\end{array}$ \\
\hline & $11 \mathrm{~h} 15-11 \mathrm{~h} 30$ & $\begin{array}{l}\text { Motivos relacionados à falência a circulação de Fontan } \\
\text { Palestrante: Fernando Antonio Fantini (MG) }\end{array}$ \\
\hline
\end{tabular}




\begin{tabular}{|c|c|c|}
\hline & 11h30- 11h45 & $\begin{array}{l}\text { Como manejar as desordens linfáticas nos pacientes com circulação e Fontan } \\
\text { Palestrante: Marcelo Biscegli Jatene (SP) }\end{array}$ \\
\hline & $11 \mathrm{~h} 45-12 \mathrm{~h}$ & $\begin{array}{l}\text { Existem evidências para mantermos os pacientes submetidos à cirurgia de Fontan } \\
\text { anticoagulados? } \\
\text { Palestrante: Vinicius José da Silva Nina (MA) }\end{array}$ \\
\hline & $12 \mathrm{~h}-12 \mathrm{~h} 15$ & $\begin{array}{l}\text { Reparo valvar nos pacientes com a anatomia univentricular. Quando? } \\
\text { Palestrante: Leonardo Augusto Miana (MG) }\end{array}$ \\
\hline $12 \mathrm{~h} 45-14 h$ & \multicolumn{2}{|c|}{ ALMOÇO/ SIMPÓSIOS } \\
\hline \multirow[t]{5}{*}{$14 h-15 h$} & \multicolumn{2}{|c|}{$\begin{array}{l}\text { SESSÃO DE TEMAS LIVRES II } \\
\text { Coordenadores: Euclides Martins Tenório (PE) } \\
\text { Fernando Antoniali (SP) }\end{array}$} \\
\hline & $14 h-14 h 15$ & $\begin{array}{l}\text { TL05 - Cirurgia cardíaca minimamente invasiva para tratamento da cardiopatia } \\
\text { congênita no adulto } \\
\text { Autor: Jerônimo Antonio Fortunato Jr (PR) } \\
\text { Comentador: Valdester C. Pinto Jr (CE) }\end{array}$ \\
\hline & $14 h 15-14 h 30$ & $\begin{array}{l}\text { TL06 - Abordagem híbrida em um único estágio de pacientes portadores de } \\
\text { Tetralogia de Fallot e atresia pulmonar com comunicação interventricular e colaterais } \\
\text { sistêmico-pulmonares } \\
\text { Autor: Marcelo Biscegli Jatene (SP) } \\
\text { Comentador: José Teles de Mendonça (SE) }\end{array}$ \\
\hline & $14 h 30-14 h 45$ & $\begin{array}{l}\text { TL } 07 \text { - Operação de Ross: seria essa a melhor opção para pacientes pediátricos? } \\
\text { Autor: Camila Roginski Guetter (PR) } \\
\text { Comentador: Marcelo Biscegli Jatene (SP) }\end{array}$ \\
\hline & $14 h 45-15 h$ & $\begin{array}{l}\text { TL } 08 \text { - Fatores de risco para troca valvar pulmonar em pacientes no pós-operatório } \\
\text { tardio de Tetralogia de Fallot } \\
\text { Autor: Maria Raquel Massoti (SP) } \\
\text { Comentador: Paulo Roberto L. Prates (RS) }\end{array}$ \\
\hline
\end{tabular}

\section{SESSÃO DE VÍDEOS}

Coordenadores: Leonardo Augusto Miana (SP)

Nilzo Augusto M. Ribeiro (BA)

\begin{tabular}{l|l|l}
\hline $15 h-16 h$ & $15 h 00-15 h 15$ & $\begin{array}{l}\text { T. BING + COAO } \\
\text { Apresentador: Bayard Gontijo Filho (MG) }\end{array}$ \\
\cline { 2 - 3 } $15 h 15-15 h 30$ & $\begin{array}{l}\text { 20 estágio abrangente pós-procedimento híbrido } \\
\text { Apresentador: Marcelo Biscegli Jatene (SP) }\end{array}$ \\
\cline { 2 - 3 } $15 h 30-15 h 45$ & $\begin{array}{l}\text { Correção de AP + Tubo VD-TP } \\
\text { Apresentador: Leonardo Augusto Miana (SP) }\end{array}$ \\
\cline { 2 - 3 } $15 h 45-16 h$ & $\begin{array}{l}\text { Troca valvar pulmonar } \\
\text { Apresentador: Andrey José de O. Monteiro (RJ) }\end{array}$
\end{tabular}

16h - 16h30 VISITA AOS EXPOSITORES E SESSÕES DE APRESENTAÇÃO DE PÔSTERES

16h30 - 17h30 Assembleia Geral do DCCVPed 


\section{- AUDITÓRI0 3}

\section{DEPARTAMENTO DE ESTIMULAÇÃO CARDÍACA ARTIFICIAL - DECA}

SIMPÓSIO DÉCIO KORMANN

\begin{tabular}{|c|c|c|}
\hline \multirow{3}{*}{$8 h-08 h 40$} & \multicolumn{2}{|c|}{$\begin{array}{l}\text { MÓDULO I } \\
\text { Caso Clínico de Marcapasso } \\
\text { Apresentador: Carlos Eduardo Duarte (SP) }\end{array}$} \\
\hline & $8 h-08 h 20$ & $\begin{array}{l}\text { Caso Clínico } 1 \\
\text { Debatedor: Giancarlo Grossi Mota (MG) }\end{array}$ \\
\hline & $8 h 20-08 h 40$ & $\begin{array}{l}\text { Caso Clínico } 2 \\
\text { Debatedor: Stela Maria Vitorino Sampaio (CE) }\end{array}$ \\
\hline \multirow{3}{*}{$08 h 40-09 h 20$} & \multicolumn{2}{|c|}{$\begin{array}{l}\text { MÓDULO II - Caso Clínico de infecção de DCEl e extração de eletrodo } \\
\text { Apresentador: Roberto Costa (SP) }\end{array}$} \\
\hline & $08 h 40-9 h$ & $\begin{array}{l}\text { Caso Clínico } 1 \\
\text { Debatedor: Luiz Paulo Rangel G. da Silva (PA) }\end{array}$ \\
\hline & $9 h-9 h 20$ & $\begin{array}{l}\text { Caso Clínico } 2 \\
\text { Debatedor: Silas dos Santos Galvão Filho (SP) }\end{array}$ \\
\hline \multirow{3}{*}{$9 h 20-10 h$} & \multicolumn{2}{|c|}{$\begin{array}{l}\text { MÓDULO III } \\
\text { Caso Clínico de terapia de ressincronização cardíaca } \\
\text { Apresentador: José Carlos Pachón Mateos (SP) }\end{array}$} \\
\hline & $9 h 2-9 h 40$ & $\begin{array}{l}\text { Caso Clínico } 1 \\
\text { Debatedor: Bráulio José Baraúna de Pinna Jr (SP) }\end{array}$ \\
\hline & $9 h 40-10 h$ & $\begin{array}{l}\text { Caso Clínico } 2 \\
\text { Debatedor: Cândido Rodrigues M. Gomes (DF) }\end{array}$ \\
\hline $10 h-10 h 45$ & \multicolumn{2}{|c|}{ VISITA AOS EXPOSITORES E SESSÕES DE APRESENTAÇÃO DE PÔSTERES } \\
\hline \multirow{3}{*}{$10 h 45-11 \mathrm{~h} 25$} & \multicolumn{2}{|c|}{$\begin{array}{l}\text { MÓDULO IV - Caso clínico de cardiodesfibrilador implantável I } \\
\text { Apresentador: Eduardo Rodrigues B. Costa (SP) }\end{array}$} \\
\hline & $10 h 45-11$ h05 & $\begin{array}{l}\text { Caso Clínico } 1 \\
\text { Debatedor: Cláudio José Fuganti (SP) }\end{array}$ \\
\hline & $11 \mathrm{~h} 05-11 \mathrm{~h} 25$ & $\begin{array}{l}\text { Caso Clínico } 2 \\
\text { Debatedor: Cândido Rodrigues M. Gomes (DF) }\end{array}$ \\
\hline \multirow{4}{*}{$11 \mathrm{~h} 25-12 \mathrm{~h} 05$} & \multicolumn{2}{|c|}{$\begin{array}{l}\text { MÓDULO V } \\
\text { Caso Clínico de cardiodesfibrilador implantável II } \\
\text { Apresentador: André Messias Guimarães (SP) }\end{array}$} \\
\hline & $11 \mathrm{~h} 25-11 \mathrm{~h} 45$ & $\begin{array}{l}\text { Caso clínico } 1 \\
\text { Debatedor: Sérgio Bronchtein (RJ) }\end{array}$ \\
\hline & $11 \mathrm{~h} 45-12 \mathrm{~h} 05$ & $\begin{array}{l}\text { Caso clínico } 2 \\
\text { Debatedor: José Carlos B. Maio Ribeiro (RJ) }\end{array}$ \\
\hline & $12 \mathrm{~h} 05-12 \mathrm{~h} 15$ & Discussão \\
\hline $12 \mathrm{~h} 15-14 \mathrm{~h}$ & \multicolumn{2}{|c|}{ Assembleia DECA } \\
\hline
\end{tabular}




\begin{tabular}{|c|c|c|}
\hline & \multicolumn{2}{|c|}{$\begin{array}{l}\text { MÓDULO VI } \\
\text { Como eu trato? Dúvidas frequentes na estimulação cardíaca } \\
\text { Coordenador: Luiz Paulo Rangel G. da Silva (PA) }\end{array}$} \\
\hline & $14 \mathrm{~h}-14 \mathrm{~h} 10$ & $\begin{array}{l}\text { Como estratificar um paciente com síncope? } \\
\text { Palestrante: Antonio Malan C. Lima (GO) }\end{array}$ \\
\hline & $14 \mathrm{~h} 10-14 \mathrm{~h} 20$ & $\begin{array}{l}\text { Quais as orientações gerais para um portador de DCEl a respeito de interferências? } \\
\text { Palestrante: Celso Salgado de Melo (MG) }\end{array}$ \\
\hline & $14 \mathrm{~h} 20-14 \mathrm{~h} 30$ & $\begin{array}{l}\text { Qual a melhor técnica para implante de MP em crianças? } \\
\text { Palestrante: Cecília M. Boya Barcellos (SP) }\end{array}$ \\
\hline & $14 \mathrm{~h} 30-14 \mathrm{~h} 40$ & $\begin{array}{l}\text { Que tipos de exercícios físicos liberar para um portadaor de CDI? } \\
\text { Palestrante: Emanoel Gledeston D. Licarião (RR) }\end{array}$ \\
\hline & $14 \mathrm{~h} 40-14 \mathrm{~h} 50$ & $\begin{array}{l}\text { Qual o candidato à TRC deveria receber um CDI? } \\
\text { Palestrante: Cláudio José Fuganti (PR) }\end{array}$ \\
\hline \multirow{7}{*}{$14 h-16 h$} & $14 h 50-15 h 0$ & $\begin{array}{l}\text { Como programar um CDI para prevenção primária? } \\
\text { Palestrante: Silas dos Santos Galvão Filho (SP) }\end{array}$ \\
\hline & $15 h-15 h 10$ & $\begin{array}{l}\text { Técnica endocárdica } x \text { toracotomia para situações de insucesso pela via seio coronário } \\
\text { Palestrante: Wilson Lopes Pereira (SP) }\end{array}$ \\
\hline & $15 h 10-15 h 20$ & $\begin{array}{l}\text { Como otimizar os intervalos AV/VV na TRC? } \\
\text { Palestrante: Antonio Vitor Moraes Jr (SP) }\end{array}$ \\
\hline & $15 h 20-15 h 30$ & $\begin{array}{l}\text { Como abordar um paciente com choques do CDI? } \\
\text { Palestrante: Antonio Carlos Assumpção (SP) }\end{array}$ \\
\hline & $15 h 30-15 h 40$ & $\begin{array}{l}\text { Estimulação cardíaca sem eletrodos: promessa ou realidade? } \\
\text { Palestrante: Álvaro Roberto B. Costa (RN) }\end{array}$ \\
\hline & $15 h 40-15 h 50$ & $\begin{array}{l}\text { Quando selecionar um paciente para CDI subcutâneo? } \\
\text { Palestrante: Genildo Ferreira Nunes (TO) }\end{array}$ \\
\hline & $15 h 50-16 h$ & Discussão \\
\hline
\end{tabular}

\section{-AUDITÓRI0 5}

\section{SIMPÓSIO DE FISIOTERAPIA EM CIRURGIA CARDIOVASCULAR}

\begin{tabular}{l|l|l}
\hline $8 h-8 h 30$ & ABERTURA \\
\hline $8 h 30-09 h 30$ & $\begin{array}{l}\text { SESSÃO TEMAS LIVRES I } \\
\text { Avaliadores: Gabriel Pinheiro (RJ), Luiz Fernando Rodrigues Jr. (RJ), Pedro Henrique Scheidt (MG), Renata Trimer (SP) }\end{array}$ \\
\hline $8 h 30-08 h 40$ & $\begin{array}{l}\text { TL } 01 \text { - O produto da distância caminhada pelo peso corporal versus distância } \\
\text { percorrida na avaliação da cinética do consumo de oxigênio em pacientes } \\
\text { coronariopatas } \\
\text { Autor: Isadora Salvador Rocco (SP) }\end{array}$ \\
\hline $8 h 40-08 h 50$ & $\begin{array}{l}\text { TL 02 - Novas perspectivas para avaliação da resistência muscular respiratória } \\
\text { baseado no limiar de carga pressórica: comparação entre dois protocolos de obtenção } \\
\text { do PthMax } \\
\text { Autor: Guilherme de Souza Areias (RJ) }\end{array}$ \\
\hline
\end{tabular}




\begin{tabular}{|c|c|c|}
\hline & $8 h 50-0 h$ & $\begin{array}{l}\text { TL } 03 \text { - A similaridade entre os valores de consumo de oxigênio obtidos pela fórmula } \\
\text { preditiva e medida pelo teste de exercício cardiopulmonar na potência do limiar } \\
\text { anaerobiose e no pico do exercício em homens jovens, adultos e portadores de } \\
\text { insuficiência cardíaca } \\
\text { Autor: Rafael Santiago Floriano (RJ) }\end{array}$ \\
\hline & $9 h-9 h 10$ & $\begin{array}{l}\text { TL } 04 \text { - Percentual de queda da distância no teste de caminhada de } 6 \text { minutos como } \\
\text { marcador prognóstico após cirurgia de revascularização do miocárdio } \\
\text { Autor: Marcela Viceconte (SP) }\end{array}$ \\
\hline & $9 h 10-9 h 20$ & $\begin{array}{l}\text { TL } 05 \text { - Limitação ao fluxo expiratório em pacientes com doença arterial coronariana } \\
\text { durante exercício de moderada e alta intensidade } \\
\text { Autor: Aparecida Maria Catai (SP) }\end{array}$ \\
\hline & $9 h 20-09 h 30$ & $\begin{array}{l}\text { TL } 06 \text { - Valor adicional do limiar anaeróbio em um modelo de predição de morte geral } \\
\text { em uma coorte urbana de pacientes com cardiopatia chagásica } \\
\text { Autor: Roberto Ribeiro da Silva (RJ) }\end{array}$ \\
\hline $9 h 30-10 h$ & \multicolumn{2}{|c|}{$\begin{array}{l}\text { CONFERÊNCIA } \\
\text { Novas tecnologias em reabilitação cardiovascular intra-hospitalar no paciente cirúrgico } \\
\text { Presidente: Michel Silva Reis (RJ) } \\
\text { Conferencista: Solange Guizilini (SP) }\end{array}$} \\
\hline $10 h-10 h 45$ & \multicolumn{2}{|c|}{ VISITA AOS EXPOSITORES E SESSÕES DE APRESENTAÇÃO DE PÔSTERES } \\
\hline 10h45-11h15 & \multicolumn{2}{|c|}{$\begin{array}{l}\text { CONFERÊNCIA } \\
\text { Reabilitação cardiovascular no paciente cirúrgico: home based } \\
\text { Presidente: Sérgio Chermont (RJ) } \\
\text { Conferencista: Audrey Borghi-Silva (SP) }\end{array}$} \\
\hline $11 \mathrm{~h} 15-12 \mathrm{~h} 15$ & \multicolumn{2}{|c|}{$\begin{array}{l}\text { DEBATE - Mobilização precoce no pós-operatório imediato de cirurgia cardiovascular } \\
\text { Moderador: Cláudia Rosa (RJ) } \\
\text { Palestrantes: Lilian Prado (RJ), Maurício Sant'Anna (RJ), Walter José Gomes (SP) }\end{array}$} \\
\hline $12 \mathrm{~h} 45-14 \mathrm{~h}$ & \multicolumn{2}{|c|}{ ALMOÇO/ SIMPÓSIOS } \\
\hline \multirow[t]{7}{*}{$14 h-15 h$} & \multicolumn{2}{|c|}{$\begin{array}{l}\text { SESSÃO DE TEMAS LIVRES II } \\
\text { Avaliadores: Gabriel Pinheiro (RJ), Luiz Fernando Rodrigues Jr. (RJ), Pedro Henrique Scheidt (MG), Renata } \\
\text { Trimmer (SP) }\end{array}$} \\
\hline & $14 h-14 h 10$ & $\begin{array}{l}\text { TL } 07 \text { - Comparação da influência da ventilação mecânica não invasiva na perfusão } \\
\text { tecidual após cirurgia de revascularização do miocárdio com CEC versus sem CEC } \\
\text { Autor: Natasha de Oliveira Marcondi (SP) }\end{array}$ \\
\hline & $14 \mathrm{~h} 10-14 \mathrm{~h} 20$ & $\begin{array}{l}\text { TL } 08 \text { - Ventilação oscilatória no exercício físico é capaz de refletir gravidade de } \\
\text { pacientes com insuficiência cardíaca crônica } \\
\text { Autor: Hugo Valverde Reis (RJ) }\end{array}$ \\
\hline & $14 h 20-14 h 30$ & $\begin{array}{l}\text { TL } 09 \text { - Comparação da força muscular respiratória no pré e pós operatório de cirurgia } \\
\text { cardiopediátrica } \\
\text { Autor: Joyce Annenberg Araújo dos Santos (AL) }\end{array}$ \\
\hline & $14 h 30-14 h 40$ & $\begin{array}{l}\text { TL } 10 \text { - Comparação da força muscular respiratória e periférica no pré e pós- } \\
\text { operatório de cirurgia cardíaca } \\
\text { Autor: Rita de Cássia dos S. Moreira (AL) }\end{array}$ \\
\hline & $14 \mathrm{~h} 40-14 \mathrm{~h} 50$ & $\begin{array}{l}\text { TL } 11 \text { - Associação entre a força muscular periférica e respiratória com a performance } \\
\text { no TIMED UP AND GO teste em idosos internados em enfermarias de cirurgia vascular } \\
\text { Autor: Jéssica Nunes Ribeiro (RJ) }\end{array}$ \\
\hline & $14 h 50-15 h$ & $\begin{array}{l}\text { TL } 12 \text { - Modulação autonômica da frequência cardíaca de pacientes com doença } \\
\text { arterial coronária inseridos em um programa de reabilitação cardiovascular } \\
\text { Autor: Glauco César da Conceição Canella (SP) }\end{array}$ \\
\hline
\end{tabular}




\begin{tabular}{|c|c|}
\hline $15 h-16 h$ & $\begin{array}{l}\text { DEBATE } \\
\text { Reabilitação baseada em exercício durante a assistência circulatória: ECMO e coração artificial } \\
\text { Moderador: Valéria Papa (SP) } \\
\text { Debatedores: Márcia Freitas (RJ), Ricardo Gaudio (RJ) }\end{array}$ \\
\hline $16 h-16 h 45$ & VISITA AOS EXPOSITORES E SESSÕES DE APRESENTAÇÃO DE PÔSTERES \\
\hline $16 h 45-17 h 15$ & $\begin{array}{l}\text { CONFERÊNCIA } \\
\text { Estratégias ventilatórias no paciente com IC aguda no pós-operatório imediato de cirurgia cardíaca } \\
\text { Presidente: Mônica Maria Pena Quintão (RJ) } \\
\text { Conferencista: Renata Trimer (SP) }\end{array}$ \\
\hline $17 h 15-17 h 45$ & $\begin{array}{l}\text { CONFERÊNCIA } \\
\text { Pós-operatório de cardiopatias congênitas complexas: particularidades no processo de reabilitar } \\
\text { Presidente: Daniele Bittencourt (RJ) } \\
\text { Conferencista: Vanessa Marques Ferreira Mendez (SP) }\end{array}$ \\
\hline $17 h 45-18 h 15$ & $\begin{array}{l}\text { DEBATE } \\
\text { Treinamento muscular respiratório no paciente cirúrgico: novas tecnologias } \\
\text { Moderador: Aparecida Maria Catai (SP) } \\
\text { Debatedores: Hugo Valverde (RS) } \\
\text { Rodrigo B. Jaenisch (RS) }\end{array}$ \\
\hline $18 \mathrm{~h} 15-18 \mathrm{~h} 30$ & Premiação dos Temas Livres \\
\hline
\end{tabular}

- AUDITÓRIO 6

\section{SIMPÓSIO DE ENFERMAGEM EM CIRURGIA CARDIOVASCULAR}

\begin{tabular}{|c|c|c|}
\hline $8 h-8 h 15-$ & \multicolumn{2}{|c|}{$\begin{array}{l}\text { ABERTURA } \\
\text { Coordenadora: Tereza Cristina Felippe Guimarães (RJ) }\end{array}$} \\
\hline \multirow{4}{*}{$8 h 15-9 h$} & \multicolumn{2}{|c|}{$\begin{array}{l}\text { SESSÃO DE TEMAS LIVRES } \\
\text { Moderadoras: Ana Carla D. Cavalcanti (RJ) } \\
\text { Maria Antonieta P. de Moraes (RS) }\end{array}$} \\
\hline & $8 h 15-8 h 30$ & $\begin{array}{l}\text { TL } 01 \text { - Aplicação de um escore de predição de risco de mediastinite em pacientes } \\
\text { submetidos a cirurgia de revascularização do miocárdio associada ou não a cirurgia de } \\
\text { troca valvar } \\
\text { Autor: Letícia Orlandin (RS) }\end{array}$ \\
\hline & $8 h 30-8 h 45$ & $\begin{array}{l}\text { TL } 02 \text { - Fatores de risco para tromboembolismo pulmonar no pós-operatório de } \\
\text { cirurgia cardíaca } \\
\text { Autor: Fernanda Lourega Chieza (RS) }\end{array}$ \\
\hline & $8 h 45-9 h$ & $\begin{array}{l}\text { TL } 03 \text { - Dispositivos de Assistência Ventricular Esquerda (LVAD): custo do curativo do } \\
\text { sítio de saída do driveline } \\
\text { Autor: Ligia Neres Matos (RJ) }\end{array}$ \\
\hline
\end{tabular}




\begin{tabular}{|c|c|c|}
\hline \multirow{3}{*}{$9 h 30-10 h$} & \multicolumn{2}{|c|}{$\begin{array}{l}\text { MESA REDONDA } \\
\text { Utilização de ECMO pós cardiotomia } \\
\text { Coordenadora: Herica Staling (RJ) }\end{array}$} \\
\hline & $9 h 30-9 h 45$ & $\begin{array}{l}\text { Na visão da Enfermagem } \\
\text { Palestrante: Lígia Neres Matos (RJ) }\end{array}$ \\
\hline & $9 h 45-10 h$ & $\begin{array}{l}\text { Na visão do Perfusionista } \\
\text { Palestrante: Carlos Araújo (PE) }\end{array}$ \\
\hline $10 h-10 h 30$ & \multicolumn{2}{|c|}{ VISITA AOS EXPOSITORES E SESSÕES DE APRESENTAÇÃO DE PÔSTERES } \\
\hline \multirow{3}{*}{$10 h 30-11 h 15$} & \multicolumn{2}{|c|}{$\begin{array}{l}\text { MESA REDONDA } \\
\text { Procedimentos percutâneos na cirurgia cardíaca: perspectivas e desafios para a enfermagem } \\
\text { Coordenadora: Rejane Reich (RS) }\end{array}$} \\
\hline & $10 h 30-10 h 50$ & $\begin{array}{l}\text { Intervenção percutânea de válvula mitral } \\
\text { Palestrante: Vanessa Silveira Faria (RJ) }\end{array}$ \\
\hline & $10 h 50-11 \mathrm{~h} 10$ & $\begin{array}{l}\text { TAVI } \\
\text { Palestrante: Debora Holanda G. de Paula (RJ) }\end{array}$ \\
\hline $11 \mathrm{~h} 10-11 \mathrm{~h} 30$ & \multicolumn{2}{|l|}{ Discussão } \\
\hline $11 \mathrm{~h} 30-12 \mathrm{~h}$ & \multicolumn{2}{|c|}{$\begin{array}{l}\text { CONFERÊNCIA } \\
\text { Segurança do paciente - Protocolos e métodos de avaliação } \\
\text { Presidente: Maria de Nazaré de S. Ribeiro (AM) } \\
\text { Conferencista: Keroulay Estebanez (RJ) }\end{array}$} \\
\hline $12 \mathrm{~h} 15-14 \mathrm{~h}$ & \multicolumn{2}{|c|}{ ALMOÇO/SIMPÓSIO SATÉLITE } \\
\hline $14 h-14 h 45$ & \multicolumn{2}{|c|}{$\begin{array}{l}\text { COLÓQUIO } \\
\text { Mobilização precoce no pós-operatório } \\
\text { Moderadora: Tamires Luciana Pena (RJ) } \\
\text { Debatedores: Médica: Jacqueline Sampaio S. Miranda (RJ) } \\
\quad \text { Enfermeira: Leticia Orlandin (RS) } \\
\quad \text { Fisioterapeuta: João Camargo (RJ) } \\
\text { 1. Quais os parâmetros clínicos para iniciar a mobilização? } \\
\text { 2. Quanto tempo depois da cirurgia, se o paciente estiver evoluindo bem poderá ser mobilizado? } \\
\text { 3. Quais os benefícios da mobilização precoce no pós-operatório? }\end{array}$} \\
\hline \multirow[t]{3}{*}{$14 h 45-15 h 15$} & \multicolumn{2}{|c|}{$\begin{array}{l}\text { MESA REDONDA } \\
\text { Acessos vasculares e terapia intravenosa na UTI de pós-operatório de cirurgia cardíaca } \\
\text { Coordenador: Jeli Zorzo (RS) }\end{array}$} \\
\hline & $14 h 45-15 h$ & $\begin{array}{l}\text { Como monitorar os acessos vasculares } \\
\text { Palestrante: Francimar Tinoco de Oliveira (RJ) }\end{array}$ \\
\hline & $15 h-15 h 15$ & $\begin{array}{l}\text { PICC: existe espaço para uso no pós operatório? } \\
\text { Palestrante: Márcia Matos (RJ) }\end{array}$ \\
\hline $15 h 15-16 h$ & \multicolumn{2}{|c|}{$\begin{array}{l}\text { COLÓQUIO } \\
\text { ACLS: quais são os desafios atuais? } \\
\text { Moderadora: Deyse Santoro da Conceição (RJ) } \\
\text { Debatedores: Juliana Faria (RJ), Lilian Bhering (RJ), Rita Simone, Lopes Moreira (SP), Maria de Nazaré de Souza } \\
\text { Ribeiro (AM) } \\
\text { 1. Implementação e adesão ao protocolo } \\
\text { 2. Treinamento do Times de resposta rápida } \\
\text { 3. Cuidados pós PCR }\end{array}$} \\
\hline $16 h-16 h 30$ & \multicolumn{2}{|c|}{ VISITA AOS EXPOSITORES E SESSÕES DE APRESENTAÇÃO DE PÔSTERES } \\
\hline
\end{tabular}




\begin{tabular}{|c|c|c|}
\hline $16 h 30-17 h$ & \multicolumn{2}{|c|}{$\begin{array}{l}\text { CONFERÊNCIA } \\
\text { Complicações neurológicas relacionadas à cirurgia cardíaca } \\
\text { Presidente: Lilian Bhering (RJ) } \\
\text { Conferencista: Patrícia Spies Subutzki (RS) }\end{array}$} \\
\hline \multirow{4}{*}{$17 h-17 h 45$} & \multicolumn{2}{|c|}{$\begin{array}{l}\text { MESA REDONDA } \\
\text { Delirium. Podemos evitar? } \\
\text { Moderador: Letícia Orlandin (RS) }\end{array}$} \\
\hline & $17 \mathrm{~h}-17 \mathrm{~h} 15$ & $\begin{array}{l}\text { Na visão do enfermeiro } \\
\text { Palestrante: Ana Paula V. Cabral (RJ) }\end{array}$ \\
\hline & 17h15-17h30 & $\begin{array}{l}\text { Na visão médica } \\
\text { Palestrante: Ana Luiza Sales (RJ) }\end{array}$ \\
\hline & $17 h 30-17 h 45$ & $\begin{array}{l}\text { Na visão da saúde mental } \\
\text { Palestrante: Natália Telles (RJ) }\end{array}$ \\
\hline $17 h 45-18 h 30$ & \multicolumn{2}{|c|}{$\begin{array}{l}\text { COLÓQUIO } \\
\text { Comunicação efetiva na UTI de pós-operatório de cirurgia cardíaca } \\
\text { Moderadora: Maria Antonieta P. de Moraes (RS) } \\
\text { Debatedores: Aline Mirema F. Vitorino (RJ), Anna Karinina Sá (RJ), Carla de Sousa Farias (RJ), Cláudia Elisabeth } \\
\text { de Almeida, (RJ), Vera Lúcia M. Pessoa (CE) } \\
\text { 1. Comunicação assertiva } \\
\text { 2. Transferência segura de informações } \\
\text { 3. Visita multiprofissional estruturada } \\
\text { 4. Feedback de performance da equipe }\end{array}$} \\
\hline
\end{tabular}

\section{- AUDITÓRI0 7}

\section{CONGRESSO BRASILEIRO DE CIRCULAÇÃO EXTRACOPÓREA DA SBCEC}

\begin{tabular}{l|l|l}
\hline $8 h-8 h 30$ & $\begin{array}{l}\text { PALESTRA DE ABERTURA E BOAS-VINDAS } \\
\text { Conferencista: Sintya T. Chalegre (PE) }\end{array}$ \\
\hline $8 h 30-10 h 30$ & $\begin{array}{l}\text { MESA REDONDA } \\
\text { Evolução e atualização das cardioplegias } \\
\text { Coordenador: Fábio Murilo Costa (MG) } \\
\text { Consultora: Vanusa Barros (RS) }\end{array}$ \\
\hline & $8 h 30-8 h 50$ & $\begin{array}{l}\text { Cardioplegias convencionais sanguíneas e microcardioplegias } \\
\text { Palestrante: Jonas dos Santos (RN) }\end{array}$ \\
\hline & $8 h 50-9 h 10$ & $\begin{array}{l}\text { Solução Del Nido clássica e modificada } \\
\text { Palestrante: Márcio Roberto do Carmo (SP) }\end{array}$ \\
\hline & $9 h 10-9 h 30$ & $\begin{array}{l}\text { Custodiol solução HTK } \\
\text { Palestrante: Ana Paula Noronha da Silva (SP) }\end{array}$ \\
\hline & $9 h 30-10 h$ & $\begin{array}{l}\text { DEBATE INTERATIVO } \\
\text { Protocolos e rotinas de serviçOS }\end{array}$ \\
\hline $\mathbf{1 0 h}-\mathbf{1 0 h} 3 \mathbf{0}$ & VISITA AOS EXPOSITORES E SESSÕES DE APRESENTAÇÃO DE PÔSTERES \\
\hline
\end{tabular}




\begin{tabular}{|c|c|c|}
\hline \multirow[t]{8}{*}{$10 h 30-12 h 15$} & \multicolumn{2}{|c|}{$\begin{array}{l}\text { SESSÃO DE TRABALHOS ORAIS } \\
\text { Coordenador: Sintya T. Chalegre (PE) } \\
\text { Consultor: Élio Barreto de Carvalho Filho (PI) }\end{array}$} \\
\hline & $10 h 30-10 h 45$ & $\begin{array}{l}\text { TT01- Padronização de serviço de circulação extracorpórea em cirurgia cardíaca } \\
\text { adulta e pediátrica } \\
\text { Autor: Alexsandro Rodrigues de Oliveira (RS) }\end{array}$ \\
\hline & $10 h 45-11 h$ & $\begin{array}{l}\text { TT } 02 \text { - Novas práticas na circulação extracorpórea: o uso da Del Nido Cardioplegia } \\
\text { Autor: Damaris Vieira Braga Carvalho (RJ) }\end{array}$ \\
\hline & $11 \mathrm{~h}-11 \mathrm{~h} 15$ & $\begin{array}{l}\text { TT } 03 \text { - Relato de caso: utilização de circuito híbrido de ECMO como ponte para o tratamento } \\
\text { Autor: Daniela Pavão (SP) }\end{array}$ \\
\hline & $11 \mathrm{~h} 15-11 \mathrm{~h} 30$ & $\begin{array}{l}\text { TT } 04 \text { - Complicações que podem ocorrer no pós-operatório de cirurgia cardiovascular } \\
\text { com circulação extracorpórea } \\
\text { Autor: Juliana Martins Provenciato (SP) }\end{array}$ \\
\hline & $11 \mathrm{~h} 30-11 \mathrm{~h} 45$ & $\begin{array}{l}\text { TT } 05 \text { - Checklist na assistência circulatória mecânica: uma ferramenta para cirurgia } \\
\text { segura } \\
\text { Autor: Rafaela Mourão R. F. Casa Nova (RJ) }\end{array}$ \\
\hline & $11 \mathrm{~h} 45-12 \mathrm{~h}$ & $\begin{array}{l}\text { TT } 06 \text { - Cuidados na circulação Extracorpórea (CEC) de pacientes com doença } \\
\text { falciforme submetidos à cirurgia cardíaca } \\
\text { Autor: Raquel Christine Kruger Miranda (RS) }\end{array}$ \\
\hline & $12 \mathrm{~h}-12 \mathrm{~h} 15$ & $\begin{array}{l}\text { TT } 07 \text { - Uso de membrana de oxigenação extracorpórea em caso de pós-transplante } \\
\text { como ponte rara para recuperação } \\
\text { Autor: Alana Michele da Rocha Melo (DF) }\end{array}$ \\
\hline $12 \mathrm{~h} 15-14 \mathrm{~h}$ & \multicolumn{2}{|c|}{ ALMOÇO/SIMPÓSIOS } \\
\hline \multirow{11}{*}{$14 \mathrm{~h} 00-16 \mathrm{~h}$} & \multicolumn{2}{|c|}{$\begin{array}{l}\text { MIN-CONFERÊNCIAS } \\
\text { DESAFIOS PROFISSIONAIS } \\
\text { Coordenador:Tonimar Monteiro da Silva (ES) } \\
\text { Consultor: Márcia Villa Nova Brasil (RS) }\end{array}$} \\
\hline & $14 h-14 h 20$ & $\begin{array}{l}\text { Concursos públicos para perfusão: equívocos no perfil exigido } \\
\text { Palestrante: Willian Duarte Machado (RS) }\end{array}$ \\
\hline & $14 \mathrm{~h} 20-14 \mathrm{~h} 25$ & Perguntas \\
\hline & $14 \mathrm{~h} 25-14 \mathrm{~h} 45$ & $\begin{array}{l}\text { Honorários da CEC e tipos de vinculação vigentes } \\
\text { Palestrante: Fábio Murilo da Costa (MG) }\end{array}$ \\
\hline & $14 \mathrm{~h} 45-14 \mathrm{~h} 50$ & Perguntas \\
\hline & $14 h 50-15 h 10$ & $\begin{array}{l}\text { Grupos de perfusionistas em prestação de serviços de CEC: como funciona e quais } \\
\text { suas vantagens } \\
\text { Palestrante: Cidenir Braga dos Santos (RJ) }\end{array}$ \\
\hline & $15 h 10-15 h 15$ & Perguntas \\
\hline & $15 h 15-15 h 35$ & $\begin{array}{l}\text { Reconhecimento da perfusão pelas categorias da área da saúde: o que está faltando? } \\
\text { Palestrante: Élio Barreto Carvalho Filho }(\mathrm{PI})\end{array}$ \\
\hline & $15 h 35-15 h 40$ & Perguntas \\
\hline & $15 h 40-16 h$ & $\begin{array}{l}\text { Diretrizes da Perfusão no Brasil: uma necessidade iminente } \\
\text { Palestrante: Márcio Roberto do Carmo (SP) }\end{array}$ \\
\hline & $16 h-16 h 05$ & Perguntas \\
\hline $16 h 05-16 h 45$ & \multicolumn{2}{|c|}{ VISITA AOS EXPOSITORES E SESSÕES DE APRESENTAÇÃO DE PÔSTERES } \\
\hline $16 h 45-17 h 45$ & \multicolumn{2}{|c|}{$\begin{array}{l}\text { Assembleia Geral Ordinária da SBCEC - ELEIÇÃO } \\
\text { Coordenadores: Sintya T. Chalegre (PE) - Presidente } \\
\text { Élio Barreto Carvalho Filho (PI) - Vice-Presidente }\end{array}$} \\
\hline
\end{tabular}




\section{- AUDITÓRIO 1}

\begin{tabular}{|c|c|c|}
\hline \multirow{7}{*}{$8 h-10 h$} & \multicolumn{2}{|c|}{$\begin{array}{l}\text { MESA REDONDA } \\
\text { VALVOPATIAS } \\
\text { Coordenadores/Coordinators: Francisco Gregori Jr. (PR), Pablo M. A. Pomerantzeff (SP) } \\
\text { Secretário/Secretary: Felipe Borsu de Sales (SP) }\end{array}$} \\
\hline & $8 h-8 h 20$ & $\begin{array}{l}\text { Cirurgia para insuficiência tricúspide secundária a hipertensão pulmonar } \\
\text { Management of Secondary Tricuspid } \\
\text { Regurgitation in CTEPH } \\
\text { Palestrante/Speaker: Michael Madani (USA) }\end{array}$ \\
\hline & $8 h 20-8 h 40$ & $\begin{array}{l}\text { Abordagem cirúrgica da insuficiência mitral isquêmica } \\
\text { Surgical approach to ischemic mitral regurgitation } \\
\text { Palestrante/Speaker: John Puskas (USA) }\end{array}$ \\
\hline & $8 h 40-9 h$ & $\begin{array}{l}\text { Há evidências de processos de melhor preservação das biopróteses? } \\
\text { Are there evidences of better preservation processes for biological valves? } \\
\text { Palestrante/Speaker: John Puskas (USA) }\end{array}$ \\
\hline & $9 h-9 h 20$ & $\begin{array}{l}\text { As biopróteses são a melhor alternativa para a válvula aórtica? } \\
\text { Are bioprosthetic valves the best alternative for patients needing AVR? } \\
\text { Palestrante/Speaker: Hartzell Schaff (USA) }\end{array}$ \\
\hline & $9 h 20-9 h 40$ & $\begin{array}{l}\text { Estenose aórtica: troca valvar ou TAVI? Panorama atual } \\
\text { The choice of TAVI or conventional aortic valve surgery in today's world } \\
\text { Palestrante/Speaker: Andreas Martens (GER) }\end{array}$ \\
\hline & $9 h 40-10 h$ & Discussão/Discussion \\
\hline $10 h-10 h 45$ & \multicolumn{2}{|c|}{ VISITA AOS EXPOSITORES E SESSÕES DE APRESENTAÇÃO DE PÔSTERES } \\
\hline \multirow{6}{*}{$10 h 45-12 h 45$} & \multicolumn{2}{|c|}{$\begin{array}{l}\text { SESSÃO DE CIRURGIA DE AORTA } \\
\text { Coordenadores/Coordinators: Carlos Eduardo P. Dantas (RJ), Ricardo Ribeiro Dias (SP) } \\
\text { Secretário/Secretary: José Kleberth Tenório Filho (AL) }\end{array}$} \\
\hline & 10h45- 11 h05 & $\begin{array}{l}\text { Breve Histórico da Cirurgia de Aorta no Brasil } \\
\text { Brief history of aortic surgery in Brazil } \\
\text { Palestrante/Speaker: Noedir Antonio G. Stolf (SP) }\end{array}$ \\
\hline & $11 \mathrm{~h} 05-11 \mathrm{~h} 25$ & $\begin{array}{l}\text { A Evolução da Cirurgia do Arco Aórtico } \\
\text { Evolution of aortic arch surgery } \\
\text { Palestrante/Speaker: Andreas Martens (GER) }\end{array}$ \\
\hline & $11 \mathrm{~h} 25-11 \mathrm{~h} 45$ & $\begin{array}{l}\text { Técnica de Bentall-DeBono para Aneurisma da Aorta Ascendente: resultados tardios } \\
\text { Late results of Bentall-DeBono procedure } \\
\text { Palestrante/Speaker: Januário Manuel de Souza (SP) }\end{array}$ \\
\hline & $11 \mathrm{~h} 45-12 \mathrm{~h} 05$ & $\begin{array}{l}\text { Técnica de David para Reparo da Aorta Ascendente: padronização atual e resultados } \\
\text { tardios } \\
\text { David operation for anulo-aortic aneurysm. Technical approach and late follow up } \\
\text { Palestrante/Speaker: Andreas Martens (GER) }\end{array}$ \\
\hline & $12 \mathrm{~h} 05-12 \mathrm{~h} 45$ & Discussão/Discussion \\
\hline
\end{tabular}




\section{-AUDITÓRIO 2}

\section{SESSÃO DE TEMAS LIVRES}

\begin{tabular}{|c|c|c|}
\hline \multirow[t]{7}{*}{$8 h-10 h$} & \multicolumn{2}{|c|}{$\begin{array}{l}\text { SESSÃO DE TEMAS LIVRES II } \\
\text { Coordenadores: Luiz Carlos Bento de Souza (SP) } \\
\text { Melchior Luiz Lima (ES) } \\
\text { Secretário: Eduardo Nunes Toniasso (SP) }\end{array}$} \\
\hline & $8 h-8 h 15$ & $\begin{array}{l}\text { TL } 09 \text { - Seleção de prótese aórtica transcateter através de software especializado é } \\
\text { mais precisa do que o Heart Team } \\
\text { Autor: Álvaro Rösler (RS) } \\
\text { Comentador: Luiz Cláudio Moreira Lima (MG) }\end{array}$ \\
\hline & $8 h 15-8 h 30$ & $\begin{array}{l}\text { TL } 10 \text { - Resultados da nova prótese transcateter INOVARE Proseal: zero de vazamento } \\
\text { perivalvar } \\
\text { Autor: Diego Felipe Gaia dos Santos (SP) } \\
\text { Comentador: Mário Coli Junqueira de Moraes (RJ) }\end{array}$ \\
\hline & $8 h 30-08 h 45$ & $\begin{array}{l}\text { TL } 11 \text { - Implante transcateter "Valve-in-valve" mitral e concomitante TAVI transapical: } \\
\text { um relato de caso para ilustrar uma mudança de paradigma } \\
\text { Autor: Leonardo Paim N. da Costa (SP) } \\
\text { Comentador: Fernando Roquette Reis Filho (MG) }\end{array}$ \\
\hline & $8 h 45-9 h$ & $\begin{array}{l}\text { TL } 12 \text { - Resultados tardios da plastia valvar mitral } \\
\text { Autor: Claudinei Collatusso (PR) } \\
\text { Comentador: Gustavo leno Judas (SP) }\end{array}$ \\
\hline & $9 h-9 h 15$ & $\begin{array}{l}\text { TL } 13 \text { - Pacientes com dissecção aguda da aorta tipo A apresentando sintomas } \\
\text { neurológicos graves, devemos operá-los? } \\
\text { Autor: Ademir Jr. Souto Jacob (RJ) } \\
\text { Comentador: Álvaro S. Albrecht (RS) }\end{array}$ \\
\hline & $9 h 15-9 h 30$ & $\begin{array}{l}\text { TL } 14 \text { - A ablação bipolar é superior à ablação unipolar no tratamento cirúrgico da } \\
\text { fibrilação atrial } \\
\text { Autor: Andrey José O. Monteiro (RJ) } \\
\text { Comentador: Flávio Donizete Gonçalves (MG) }\end{array}$ \\
\hline $10 h-10 h 45$ & \multicolumn{2}{|c|}{ VISITA AOS EXPOSITORES E SESSÕES DE APRESENTAÇÃO DE PÔSTERES } \\
\hline $10 h 45-12 h 45$ & \multicolumn{2}{|c|}{$\begin{array}{l}\text { SESSÃO DE TEMAS LIVRES III } \\
\text { Coordenadores: Alfredo Inácio Fiorelli (SP), Juan Alberto C. Mejia (CE) } \\
\text { Secretário: Fabiano Silva C. de S. Amaral (SP) }\end{array}$} \\
\hline & $10 h 45-11 h$ & $\begin{array}{l}\text { TL } 17 \text { - Resultados em longo prazo da Cirurgia de Jatene } \\
\text { Autor: Camila Lino Martins R. da Silva (SP) } \\
\text { Comentador: Jefferson Duarte F. Magalhães (RJ) }\end{array}$ \\
\hline & $11 \mathrm{~h}-11 \mathrm{~h} 15$ & $\begin{array}{l}\text { TL } 18 \text { - Cirurgia minimamente invasiva - Experiência inicial no tratamento de lesões } \\
\text { valvares } \\
\text { Autor: Olívio Alves de Souza Neto (RJ) } \\
\text { Comentador: Christiano da Silveira de Barcellos (RS) }\end{array}$ \\
\hline
\end{tabular}




\begin{tabular}{|c|c|}
\hline $11 \mathrm{~h} 15-11 \mathrm{~h} 30$ & $\begin{array}{l}\text { TL } 19 \text { - Uso de nanoemulsões lipídicas como veículos de paclitaxel e de metotrexato } \\
\text { no tratamento da doença vascular do coração transplantado em coelhos } \\
\text { Autor: Lucas Regatieri Barbieri (SP) } \\
\text { Comentador: Alfredo Inácio Fiorelli (SP) }\end{array}$ \\
\hline $11 \mathrm{~h} 30-11 \mathrm{~h} 45$ & $\begin{array}{l}\text { TL } 20 \text { - Prevalence and clinical outcomes of hyperglycemia in the perioperative period of } \\
\text { cardiac surgery according to diabetes status } \\
\text { Autor: Mauro Ricardo N. Pontes (RS) } \\
\text { Comentador: Divino Francisco Pinto (RJ) }\end{array}$ \\
\hline $11 h 45-12 h$ & $\begin{array}{l}\text { TL } 21 \text { - Impacto da avaliação renal na morbimortalidade após cirurgia cardíaca: } \\
\text { creatinina sérica é suficiente? } \\
\text { Autor: Camila Perez de S. Arthur (SP) } \\
\text { Comentador: Odilon Nogueira Barbosa (RJ) }\end{array}$ \\
\hline $12 h-12 h 15$ & $\begin{array}{l}\text { TL } 22 \text { - Emprego da ultrassonografia com doppler para mapeamento de veia safena na } \\
\text { obtenção de enxertos para cirurgia de revascularização do miocárdio } \\
\text { Autor: Fillipe Campos Lopes (SP) } \\
\text { Comentador: José Oscar Reis Brito (RJ) }\end{array}$ \\
\hline $12 \mathrm{~h} 15-12 \mathrm{~h} 30$ & $\begin{array}{l}\text { TL } 23 \text { - Biopróteses de liberação rápida. Uma mudança de paradigma. Experiência do ICP } \\
\text { Autor: Euclides Martins Tenório (PE) } \\
\text { Comentador: Darteson da Silveira Gutierrez (RJ) }\end{array}$ \\
\hline $12 \mathrm{~h} 30-12 \mathrm{~h} 45$ & $\begin{array}{l}\text { TL } 24 \text { - Hemotransfusão em cirurgia cardíaca e sua relação com desfechos adversos em } \\
\text { pós-operatório precoce } \\
\text { Autor: Ana Paula Tagliari (RS) } \\
\text { Comentador: Júlio Faria Honório (RJ) }\end{array}$ \\
\hline
\end{tabular}

\section{- AUDITÓRIO 3}

\section{RESPOSTAS CURTAS PARA QUESTÕES RELEVANTES}

\begin{tabular}{l|l|l|}
\hline $8 h-10 h$ & \multicolumn{2}{|l|}{$\begin{array}{l}\text { RESPOSTAS CURTAS PARA QUESTÕES RELEVANTES I } \\
\text { Coordenadores: Jarbas J. Dinkhuysen (SP), Paulo Roberto S. Brofman (PR) } \\
\text { Secretário: Rafaela da Hora Sales (AL) }\end{array}$} \\
\hline & $8 \mathrm{~h}-8 \mathrm{~h} 10$ & $\begin{array}{l}\text { 1. Qual é a evidência clínica da eficácia de tratamento anticalcificação nas biopróteses? } \\
\text { Palestrante: Eduardo Sérgio Bastos (RJ) }\end{array}$ \\
\hline & $8 h 10-8 \mathrm{~h} 20$ & $\begin{array}{l}\text { 2. Adulto com insuficiência valvar pulmonar em p.o. tardio de Tetralogia de Fallot: } \\
\text { quando indicar reoperação? } \\
\text { Palestrante: Andrey José O. Monteiro (RJ) }\end{array}$ \\
\hline & $8 h 20-8 h 30$ & $\begin{array}{l}\text { 3. Métodos de proteção pulmonar em cirurgias cardíacas } \\
\text { Palestrante: Paulo Manuel Pêgo-Fernandes (SP) }\end{array}$ \\
\hline & $8 h 30-8 h 40$ & $\begin{array}{l}\text { 4. Métodos de proteção renal em cirurgias cardíacas } \\
\text { Palestrante: Marcelo Sávio da S. Martins (RJ) }\end{array}$ \\
\hline & $8 h 40-8 h 50$ & $\begin{array}{l}\text { 5. Cirurgia híbrida de coronárias: tem indicação? Quando? } \\
\text { Palestrante: Pedro Rafael Salerno (PE) }\end{array}$ \\
\hline & $\begin{array}{l}\text { 6. O tratamento cirúrgico ou ablação por cateter produziu um resultado superior ao } \\
\text { medicamentoso quando nos reportamos à Fibrilação Atrial? } \\
\text { Palestrante: Magaly Arrais dos Santos (SP) }\end{array}$ \\
\hline
\end{tabular}




\begin{tabular}{|c|c|c|}
\hline & $9 h-9 h 10$ & $\begin{array}{l}\text { 7. Conduta frente a hematoma intramural em aorta descendente como achado } \\
\text { ocasional em candidato à CRM } \\
\text { Palestrante: Rubens Giambroni Filho (SP) }\end{array}$ \\
\hline & $9 h 10-9 h 20$ & $\begin{array}{l}\text { 8. Plastia valvar na insuficiência aórtica: seleção de casos e melhores técnicas } \\
\text { Palestrante: José Teles de Mendonça (SE) }\end{array}$ \\
\hline & $9 h 20-9 h 30$ & $\begin{array}{l}\text { 9. Válvula aórtica sem sutura: tem indicação e tem futuro? } \\
\text { Palestrante: Emir Kabil (Bósnia) }\end{array}$ \\
\hline & $9 h 30-9 h 40$ & $\begin{array}{l}\text { 10. Válvula aórtica bicúspide, quando indicar plástica ou troca e o tratamento da aorta } \\
\text { ascendente } \\
\text { Palestrante: Vinicius José da S. Nina (MA) }\end{array}$ \\
\hline & $9 h 40-9 h 50$ & $\begin{array}{l}\text { 11. DVSVD com estenose pulmonar: inovações cirúrgicas } \\
\text { Palestrante: Divino Francisco Pinto (RJ) }\end{array}$ \\
\hline & $9 h 50-10 h$ & $\begin{array}{l}\text { 12. Novas opções para a correção da TGA e variantes } \\
\text { Palestrante: Bayard Gontijo Filho (MG) }\end{array}$ \\
\hline $10 h-10 h 45$ & \multicolumn{2}{|c|}{ VISITA AOS EXPOSITORES E SESSÕES DE APRESENTAÇÃO DE PÔSTERES } \\
\hline \multirow{13}{*}{$10 h 45-12 h 45$} & \multicolumn{2}{|c|}{$\begin{array}{l}\text { RESPOSTAS CURTAS PARA QUESTÕES RELEVANTES II } \\
\text { Coordenadores: Marcelo Sávio da S. Martins (RJ), Rodrigo Mussi Milani (PR) } \\
\text { Secretário: Thiago Gonçalves da Silveira (SP) }\end{array}$} \\
\hline & $10 h 45-10 h 55$ & $\begin{array}{l}\text { 13. TAVI: quando os cirurgiões irão fazer a via transfemoral? } \\
\text { Palestrante: Mário Ricado Amar (RJ) }\end{array}$ \\
\hline & $10 h 55-11$ h05 & $\begin{array}{l}\text { 14. Conduta frente a achado de nódulo de aspecto maligno na face externa do } \\
\text { pericárdio em paciente de CRM com história prévia de carcinoma de mama } \\
\text { Palestrante: Eduardo da Costa Rodrigues (RJ) }\end{array}$ \\
\hline & $11 \mathrm{~h} 05-11 \mathrm{~h} 15$ & $\begin{array}{l}\text { 15. Revascularização sem CEC: é o método preferencial, por quê? } \\
\text { Palestrante: Jefferson Cavalcanti Chaves (RJ) }\end{array}$ \\
\hline & $11 \mathrm{~h} 15-11 \mathrm{~h} 25$ & $\begin{array}{l}\text { 16. Revascularização sem CEC: NÃO é o método preferencial, por quê? } \\
\text { Palestrante: Marcelo Sávio da S. Martins (RJ) }\end{array}$ \\
\hline & $11 \mathrm{~h} 25-11 \mathrm{~h} 35$ & $\begin{array}{l}\text { 17. A robótica com todo seu aparato mudou o cenário do resultado cirúrgico, maior } \\
\text { objetivo de todo cirurgião? } \\
\text { Palestrante: Robinson Poffo (SC) }\end{array}$ \\
\hline & $11 \mathrm{~h} 35-11 \mathrm{~h} 45$ & $\begin{array}{l}\text { 18. As cirurgias minimamente invasivas transformaram positivamente a mortalidade } \\
\text { cirúrgica? Onde? } \\
\text { Palestrante: Olívio Alves Souza Neto (RJ) }\end{array}$ \\
\hline & $11 \mathrm{~h} 45-11 \mathrm{~h} 55$ & $\begin{array}{l}\text { 19. Intervenção coronária percutânea prévia influi no resultado da revascularização } \\
\text { cirúrgica? } \\
\text { Palestrante: Luís Sérgio de M. Fragomeni (RS) }\end{array}$ \\
\hline & $11 \mathrm{~h} 55-12 \mathrm{~h} 05$ & $\begin{array}{l}\text { 20. Aneurisma de arco aórtico: híbrido ou convencional? } \\
\text { Palestrante: Valdo José Carreira (RJ) }\end{array}$ \\
\hline & $12 \mathrm{~h} 05-12 \mathrm{~h} 15$ & $\begin{array}{l}\text { 21. Dissecção do tipo B não complicada: tratamento clínico conservador ou } \\
\text { intervenção endovascular para todos? } \\
\text { Palestrante: José Honório de A. Palma da Fonseca (SP) }\end{array}$ \\
\hline & $12 \mathrm{~h} 15-12 \mathrm{~h} 25$ & $\begin{array}{l}\text { 22. Cirurgia da aorta ascendente: preservar ou trocar a valva aórtica? } \\
\text { Palestrante: Eduardo Augusto Victor Rocha (MG) }\end{array}$ \\
\hline & $12 \mathrm{~h} 25-12 \mathrm{~h} 35$ & $\begin{array}{l}\text { 23. Como incrementar a assistência circulatória mecânica no Brasil? } \\
\text { Palestrante: Alexandre S. Colafranceschi (RJ) }\end{array}$ \\
\hline & $12 \mathrm{~h} 35-12 \mathrm{~h} 45$ & $\begin{array}{l}\text { 24. Uso de sangue em cirurgia cardíaca: Como evitar e quando é indispensável? } \\
\text { Palestrante: Mário Coli Junqueira de Moraes (RJ) }\end{array}$ \\
\hline
\end{tabular}




\section{-AUDITÓRIO 4}

\section{CURSO DE TAVI}

Coordenadores: Paulo Roberto L. Prates (RS), Rui M. S. Almeida (PR)

\begin{tabular}{l|l}
\hline $8 \mathrm{~h}-8 \mathrm{~h} 20$ & $\begin{array}{l}\text { Como iniciar um programa de TAVI? } \\
\text { Palestrante: Magaly Arrais dos Santos (SP) } \\
\text { Comentador: Fernando A. Lucchese (RS) }\end{array}$ \\
\hline $8 \mathrm{~h} 20-8 \mathrm{~h} 40$ & $\begin{array}{l}\text { Clinicals Trials Updates: resultados dos últimos ensaios clínicos } \\
\text { Palestrante: Eduardo Keller Saadi (RS) } \\
\text { Comentador: José Honório de A. Palma da Fonseca (SP) }\end{array}$ \\
\hline $8 \mathrm{~h} 40-9 \mathrm{~h}$ & $\begin{array}{l}\text { Dificuldades anatômicas e complicações: alternativas e tratamento } \\
\text { Palestrante: Marcela da Cunha Sales (RS) } \\
\text { Comentador: Marcus Vinicius N. Santos (DF) }\end{array}$ \\
\hline 9h-9h20 & $\begin{array}{l}\text { Programa de Residência Médica em Cirurgia Cardiovascular: como tornar TAVI uma rotina? } \\
\text { Palestrante: Paulo Roberto L. Prates (RS) } \\
\text { Comentador: Rui M. S. Almeida (PR) }\end{array}$ \\
\hline 9h20-9h40 & $\begin{array}{l}\text { TAVI: o que o futuro nos reserva? } \\
\text { Palestrante: Diego Felipe Gaia dos Santos (SP) } \\
\text { Comentador: Rodrigo de Castro Bernardes (MG) }\end{array}$ \\
\hline 9h40 - 10h & Discussão \\
\hline $\mathbf{1 0 h}-\mathbf{1 0 h 4 5}$ & VISITA AOS EXPOSITORES E SESSÕES DE APRESENTAÇÃO DE PÔSTERES
\end{tabular}

\section{-AUDITÓRIO 4}

CURSO DE TEVAR

Tratamento Endovascular das doenças da aorta torácica

Coordenadores: Eduardo K. Saadi (RS), Christiano da Silveira de Barcellos (RS)

Secretário: Ricardo Langenneger (RS)

\begin{tabular}{|c|c|c|}
\hline \multirow{8}{*}{$10 h 45-12 h 45$} & 10h45-11h05 & $\begin{array}{l}\text { Materiais básicos necessários para procedimentos endovasculares da aorta (fios guias, } \\
\text { catéteres, introdutores etc) } \\
\text { Palestrante: Dr. José Honório A. Palma da Fonseca (SP) }\end{array}$ \\
\hline & $11 \mathrm{~h} 05-11 \mathrm{~h} 15$ & Discussão \\
\hline & 11h15-11h35 & $\begin{array}{l}\text { Passo a passo na correção endovascular dos aneurismas/dissecções da aorta torácica } \\
\text { descendente } \\
\text { Palestrante: Andrey José de O. Monteiro (RJ) }\end{array}$ \\
\hline & $11 \mathrm{~h} 35-11 \mathrm{~h} 45$ & Discussão \\
\hline & $11 \mathrm{~h} 45-12 \mathrm{~h} 05$ & $\begin{array}{l}\text { Indicações e resultados atuais do TEVAR em aneurismas da aorta torácica e dissecções } \\
\text { Palestrante: Diego Felipe Gaia dos Santos (SP) }\end{array}$ \\
\hline & $12 \mathrm{~h} 05-12 \mathrm{~h} 15$ & Discussão \\
\hline & $12 \mathrm{~h} 15-12 \mathrm{~h} 35$ & $\begin{array}{l}\text { Casos editados (com diferentes dispositivos) } \\
\text { Apresentador: Eduardo Keller Saadi (RS) }\end{array}$ \\
\hline & $12 \mathrm{~h} 35-12 \mathrm{~h} 45$ & Discussão \\
\hline
\end{tabular}




\section{-AUDITÓRI0 5}

\section{SIMPÓSIO DE FISIOTERAPIA EM CIRURGIA CARDIOVASCULAR}

HANDS-ON 1 - Ventilação mecânica no pós-operatório de cirurgia cardíaca

ESTAÇÃO 1 - Princípios de ventilação mecânica

Douglas Willian Bolzan Lima (SP)

Solange Guizilini (SP)

ESTAÇÃO 2 - Ventilação mecânica avançada - Estratégias no SDRA e ECMO

Marcus Vinicius Souza (RJ)

8h - 12h30 Vanessa Marques Ferreira Mendez (SP)

\section{ESTAÇÃO 3 - Ventilação não-invasiva}

Beatriz Robert Moreira (RJ)

Carla Cristiane Santos Soares (RJ)

ESTAÇÃO 4 - Ventilação mecânica em neonatologia/pediatria

Fellipe Allevato (RJ)

Vanessa Marques Ferreira Mendez (SP)

HANDS-ON 2 - Princípios de avaliação e prescrição de exercícios físicos na reabilitação cardíaca no pré e pós-operatório de cirurgia cardíaca

\section{ESTAÇÃO 1 - Avaliação cardiopulmonar}

Aparecida Maria Catai (SP)

$8 h-12 h 30 \quad$ Audrey Borghi-Silva (SP)

Michel Silva Reis (RJ)

ESTAÇÃO 2 - Princípios de prescrição de exercícios

Maurício Sant'Anna (RJ)

Paloma Hargraves (RJ)

\section{-AUDITÓRIO 6}

\section{SIMPÓSIO DE ENFERMAGEM EM CIRURGIA CARDIOVASCULAR}

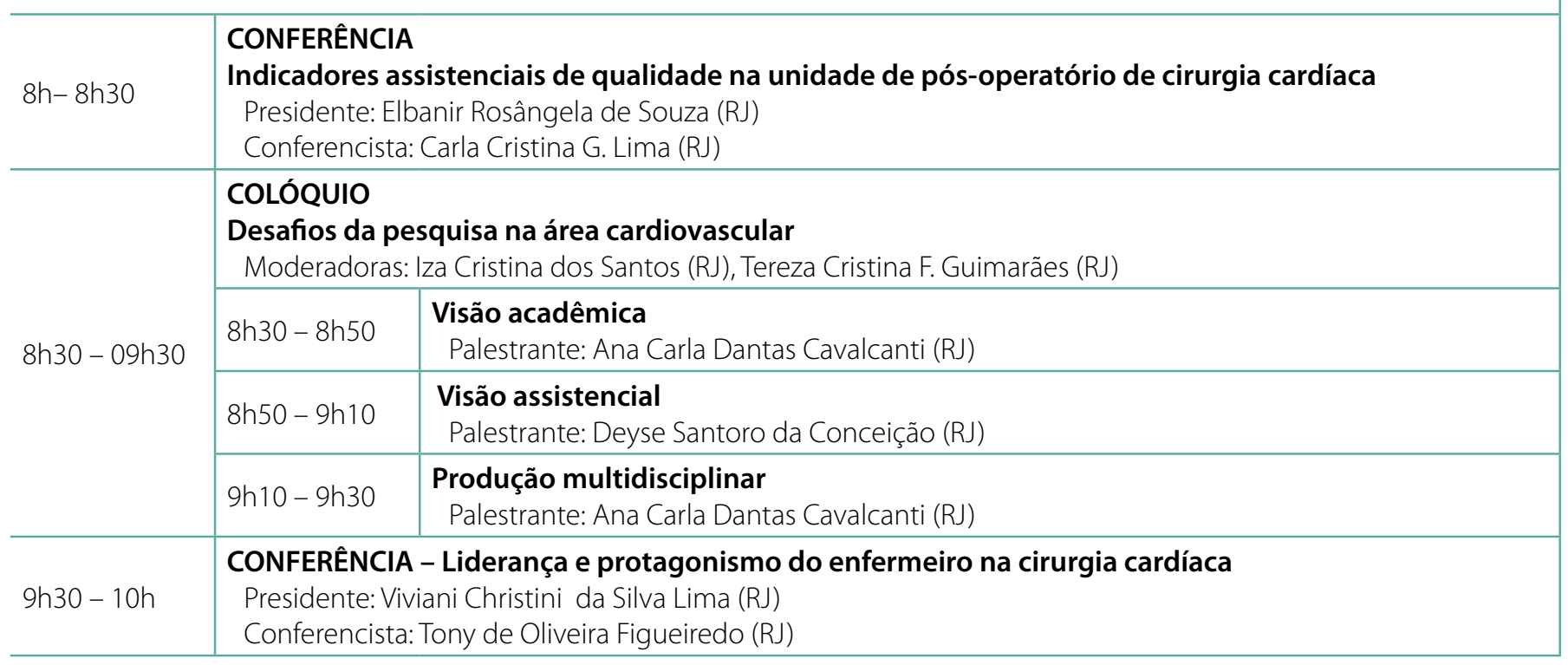




\begin{tabular}{|c|c|c|}
\hline $10 h-10 h 30$ & \multicolumn{2}{|c|}{ VISITA AOS EXPOSITORES E SESSÕES DE APRESENTAÇÃO DE PÔSTERES } \\
\hline \multirow{4}{*}{$10 h 30-11 \mathrm{~h} 15$} & \multicolumn{2}{|c|}{$\begin{array}{l}\text { MESA REDONDA - Como formar uma equipe de ultracomplexidade na cirurgia cardíaca? } \\
\text { Coordenadora: Vera Lúcia M. Pessoa (CE) }\end{array}$} \\
\hline & $10 h 30-10 h 50$ & $\begin{array}{l}\text { Na visão do gestor } \\
\text { Palestrante: Alexandre S. Colafranceschi (RJ) }\end{array}$ \\
\hline & $10 h 50-11 h 10$ & $\begin{array}{l}\text { Na visão assistencial } \\
\text { Palestrante: Tereza Cristina F. Guimarães (RJ) }\end{array}$ \\
\hline & $11 \mathrm{~h} 10-11 \mathrm{~h} 15$ & Discussão \\
\hline $11 \mathrm{~h} 30-12 \mathrm{~h}$ & \multicolumn{2}{|c|}{$\begin{array}{l}\text { CONFERÊNCIA - Modelos de gestão em uma unidade cardiointensiva } \\
\text { Presidente: Lourdes Alexandrina de C. Neves (RJ) } \\
\text { Conferencista: Luciana Cristina Lima Correia (RJ) }\end{array}$} \\
\hline
\end{tabular}

\section{- AUDITÓRIO 7}

\section{CONGRESSO BRASILEIRO DE CIRCULAÇÃO EXTRACOPÓREA DA SBCEC}

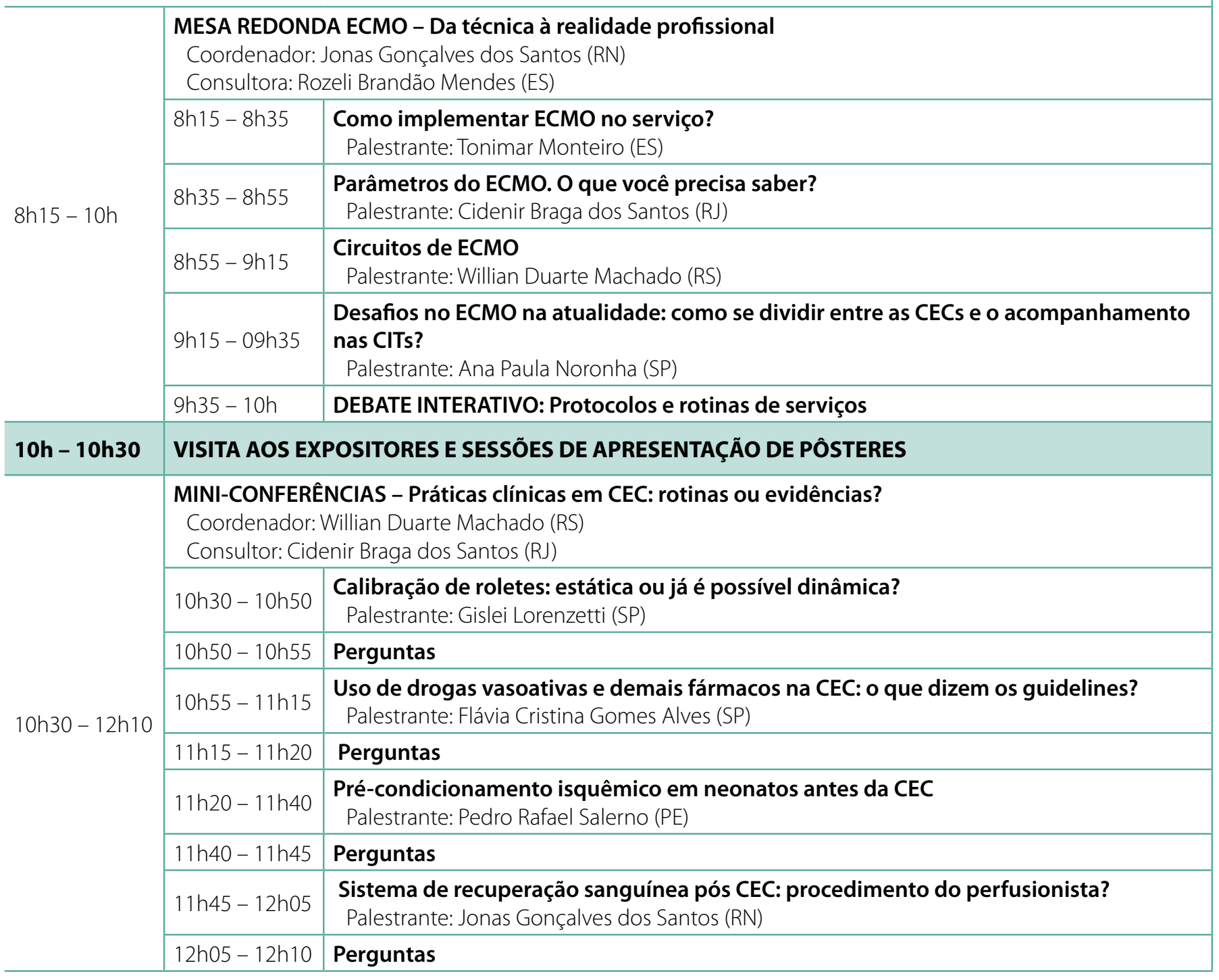


Entrega dos Títulos de Especialista em Perfusão, dos Prêmios do Congresso SBCEC e Foto Oficial 2017 Coordenadores: Sintya T. Chalegre (PE)

Élio Barreto Carvalho Filho (PI)

12h10 - $12 \mathrm{~h} 40$ Melhor Estudo Clínico: Prêmio Antonio Gilberto Martins

Melhor Relato de Caso: Prêmio Otoni Moreira Gomes

Melhor Trabalho Experimental: Prêmio Domingo Marcolino Braile

Melhor Revisão Sistemática: Prêmio Maria Helena Leal de Souza

14h-17h Prova do Título de Especialista em Perfusão

\section{- AUDITÓRI0 8}

\begin{tabular}{|l|l|l|}
\hline \multirow{2}{*}{$8 h-10 h$} & $\begin{array}{l}\text { CURSO DE ECOCARDIOGRAFIA INTRAOPERATÓRIA } \\
\text { Coordenadores: Mário Ricardo Amar (RJ) } \\
\text { Olívio A. Souza Neto (RJ) }\end{array}$ \\
\cline { 2 - 3 } $8 \mathrm{~h}-8 \mathrm{~h} 20$ & $\begin{array}{l}\text { Quantificação da válvula mitral no intraoperatório } \\
\text { Palestrante: Eduardo S. L. Botelho (RJ) }\end{array}$ \\
\cline { 2 - 3 } & $8 h 20-8 h 40$ & $\begin{array}{l}\text { Avaliação intraoperatória do VD } \\
\text { Palestrante: Eduardo S. L. Botelho (RJ) }\end{array}$ \\
\cline { 2 - 3 } & $8 h 40-9 h$ & $\begin{array}{l}\text { Reparo valvar aórtico em cirurgias da raiz aórtica } \\
\text { Palestrante: Alexandre Fernandes (RJ) }\end{array}$ \\
\cline { 2 - 3 } & $9 h-9 h 20$ & $\begin{array}{l}\text { Implante transcateter da válvula aórtica (TAVI) } \\
\text { Palestrante: Marcello Salgado Filho (RJ) }\end{array}$ \\
\cline { 2 - 3 } & $9 h 20-10 h$ & Discussão \\
\hline \multirow{2}{*}{$\mathbf{1 0 h}-\mathbf{1 0 h 4 5}$} & VISITA AOS EXPOSITORES E SESSÕES DE APRESENTAÇÃO DE PÔSTERES \\
\hline
\end{tabular}

\section{- AUDITÓRIO 8}

\begin{tabular}{c|l|l}
$10 h 45-12 \mathrm{~h} 45$ & $\begin{array}{l}\text { CURSO DE CIRURGIA DA HIPERTENSAO PULMONAR POR TROMBOEMBOLISMO CRONICO (HPTEC) } \\
\text { Coordenadores: Álvaro S. Albrecht (RS) } \\
\text { Gisela B. Meyer (RS) }\end{array}$ \\
\hline \multirow{1}{*}{$10 \mathrm{~h} 45-11 \mathrm{hh05}$} & $\begin{array}{l}\text { Da embolia pulmonar aguda (TEP) à HPTEC } \\
\text { Palestrante: Marcelo Luiz da S. Bandeira (RJ) } \\
\text { Objetivo: apresentar a epidemiologia e pato-fisiologia do HPTEC, responder aos seguintes } \\
\text { questionamentos: podemos prevenir a HPTEC após TEP agudo? }\end{array}$ \\
\hline $11 \mathrm{~h} 05-11 \mathrm{~h} 25$ & $\begin{array}{l}\text { Diagnóstico e imagem: onde a imagem ajuda? } \\
\text { Palestrante: Rosana S. Rodrigues (RJ)) } \\
\text { Objetivo: Apresentar o diagnóstico por imagem de HPTEC, responder aos seguintes } \\
\text { questionamentos: quais os diagnósticos diferenciais nos quais as imagens auxiliam? Como } \\
\text { definir pacientes candidatos à cirurgia? O que há de novo em método de imagem? }\end{array}$ \\
\hline \multirow{1}{*}{$11 \mathrm{~h} 25-11 \mathrm{~h} 45$} & $\begin{array}{l}\text { Endarterectomia pulmonar. O estado da arte } \\
\text { Pulmonary thromboendarterectomy. State of Art } \\
\text { Palestrante/Speaker: Michael Madani (USA) } \\
\text { Ojetivo: Apresentar as principais características da cirurgia de endarterectomia pulmonar, } \\
\text { como indicar, requisitos básicos e técnica cirúrgica - apresentar detalhes técnicos. }\end{array}$ \\
\hline
\end{tabular}




\begin{tabular}{l|l|l|}
\hline $11 \mathrm{~h} 45-12 \mathrm{~h} 05$ & $\begin{array}{l}\text { Manejo pré e trans operatório. O que a anestesia precisa saber? } \\
\text { Palestrante: Diogo Centenaro (RS) } \\
\text { Objetivo: apresentar as principais características da seleção e cuidados pré e trans } \\
\text { operatórios peculiares à cirurgia do HPTEC }\end{array}$ \\
\hline \multirow{2}{*}{$12 \mathrm{~h} 05-12 \mathrm{~h} 25$} & $\begin{array}{c}\text { Tratamento clínico e seleção de casos para cirurgia } \\
\text { Palestrante: Gisela M. Meyer (RS) } \\
\text { Objetivo: Apresentar as principais indicações para a opção pelo tratamento } \\
\text { medicamentoso, resultados dos últimos estudos e a prática do uso da terapia } \\
\text { medicamentosa através de casos clínicos. }\end{array}$ \\
\hline $12 \mathrm{~h} 25-12 \mathrm{~h} 45$ & $\begin{array}{l}\text { Endarterectomia pulmonar - até onde iremos? } \\
\text { Palestrante/Speaker: Michael Madani (EUA) } \\
\text { Objetivo: apresentar situações limítrofes da indicação cirúrgica - mitos e verdades. } \\
\text { Responder aos seguintes questionamentos: Há limite de idade para a cirurgia? Quais as } \\
\text { morbidades são contra-indicações absolutas? Qual é o limite distal de operabilidade? }\end{array}$ \\
\hline $12 \mathrm{~h} 45-12 \mathrm{~h} 55$ & \begin{tabular}{l} 
Perguntas e discussão \\
\hline
\end{tabular} \\
\hline
\end{tabular}

\section{- AUDITÓRIO 9}

\section{SIMPÓSIO DE DEFESA PROFISSIONAL}

Coordenadores: José Wanderley Neto (AL), Marcelo Matos Cascudo (RN)

Debatedores: Haroldo Adans Ferraz (RJ), Rodrigo Minati Barbosa (RJ)

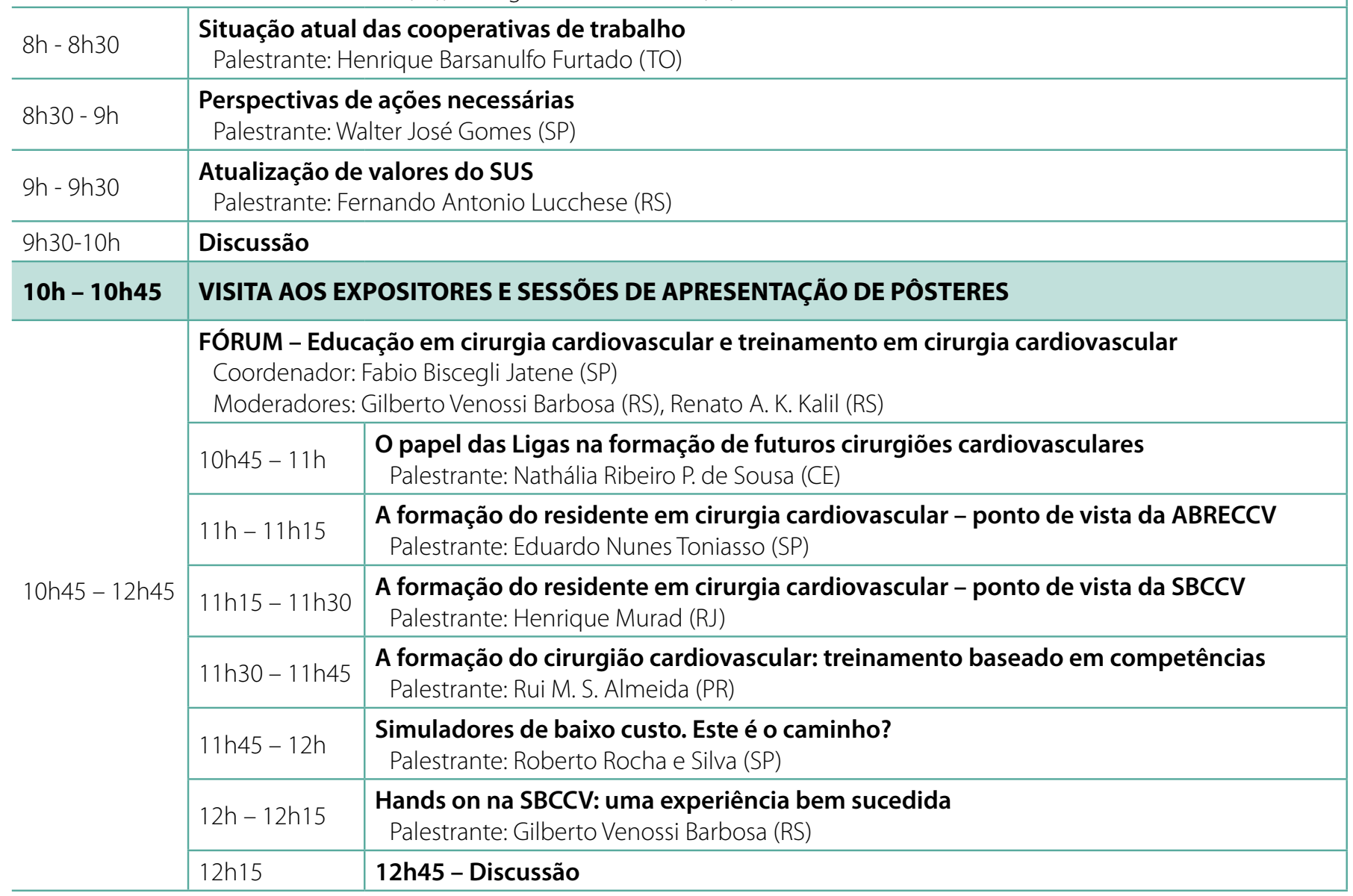




\begin{tabular}{|c|c|c|}
\hline & BLOCO 1A & BLOCO 1B \\
\hline $7 \mathrm{~h}$ & \multicolumn{2}{|c|}{ CREDENCIAMENTO } \\
\hline 9h10h30 & $\begin{array}{l}\text { MÓDULO I A } \\
\text { ANULOPLASTIA DA VALVA MITRAL COM ANÉIS } \\
\text { E RESSECÇÃO DA AURICULETA ESQUERDA } \\
\text { Apresentadora: Magaly Arrais dos Santos (SP) } \\
\text { Experts: Euclides Martins Tenório (PE), José Dario Frota Filho (RS), } \\
\text { José Oscar Reis Brito (RJ), Marco Antônio V. Guedes (BA) }\end{array}$ & $\begin{array}{l}\text { MÓDULO I B } \\
\text { ANULOPLASTIA DA VALVA MITRAL COM ANEL/ } \\
\text { BANDA MINIMAMENTE INVASIVA ATRAVÉS DE } \\
\text { ACESSO POR MINI TORACOTOMIA } \\
\text { Apresentador: Francisco Diniz A. da Costa (PR) } \\
\text { Experts: Carlos Manuel de A. Brandão (SP), Rodrigo M. Milani (PR), } \\
\text { Stêvan Krieger Martins(SP), Wanewman Lins G. de Andrade (BA) }\end{array}$ \\
\hline 10h/10h30 & \multicolumn{2}{|c|}{ INTERVALO E VISITAÇÃO EXPOSIÇÃO PARALELA } \\
\hline 10h30/12h & $\begin{array}{l}\text { MÓDULO II A } \\
\text { TRANSPLANTE CARDÍACO BICAVAL } \\
\text { Apresentador: Juan Alberto C. Mejia (CE) } \\
\text { Experts: Carlos Eduardo Pereira Dantas (RJ), Claudinei Collatusso } \\
\text { (PR), Cláudio Leo Gelape (MG), Jarbas J. Dinkhuysen (SP) }\end{array}$ & $\begin{array}{l}\text { MÓDULO II B } \\
\text { CIRURGIA DE BENTAL DE BONO } \\
\text { Apresentador: Ricardo Ribeiro Dias (SP) } \\
\text { Experts: Andrea Dumsch de A. Ferreira (PR), Marcela da Cunha } \\
\text { Sales (RS), Nelson Barg (RJ), Valdo José Carreira (RJ) }\end{array}$ \\
\hline $12 \mathrm{~h} / 13 \mathrm{~h}$ & \multicolumn{2}{|c|}{ INTERVALO E VISITAÇÃO EXPOSIÇÃO PARALELA/ ALMOÇO (LIVRE) } \\
\hline 13h/14h30 & $\begin{array}{l}\text { MÓDULO III A } \\
\text { TRATAMENTO DA FIBRILAÇÃO ATRIAL POR } \\
\text { ISOLAMENTO CIRÚRGICO DAS VEIAS PULMONARES } \\
\text { Apresentador: Renato Abdala Karam Kalil (RS) } \\
\text { Experts: Álvaro Albrecht (RS), Andrey José de O. Monteiro (RJ), } \\
\text { Flávio Donizete Gonçalves (MG), José Wanderley Neto (AL) }\end{array}$ & $\begin{array}{l}\text { MÓDULO III B } \\
\text { PLASTIA VÍDEO-ASSISTIDA DA VALVA MITRAL } \\
\text { Apresentador: Robinson Poffo (SP) } \\
\text { Experts: Carlos Manuel de A. Brandão (SP), Daniel Peres } \\
\text { Guimarães (SP), Jerônimo A. Fortunato Jr (PR), Júlio Faria Honório } \\
\text { (SP), Melchior Luiz Lima (ES), Olívio Alves Souza Neto (RS), Ricardo } \\
\text { Barros Corso (DF) }\end{array}$ \\
\hline 16h/16h30 & \multicolumn{2}{|c|}{ INTERVALO E VISITAÇÃO EXPOSIÇÃO PARALELA } \\
\hline 14h30/16h & $\begin{array}{l}\text { MÓDULO IV A } \\
\text { CRM COM AMBAS ARTÉRIAS MAMÁRIAS } \\
\text { Apresentador: Alexandre C. Hueb (SP) } \\
\text { Experts: Josalmir José M. do Amaral (RN), Márcio José Paes (SC), } \\
\text { Maurílio Onofre Deininger (PB), Rodrigo Segalote (RJ) }\end{array}$ & $\begin{array}{l}\text { MÓDULO IV B } \\
\text { ATRIOSSEPTOPLASTIA VÍDEO-ASSISTIDA } \\
\text { Apresentador: Ricardo Barros Corso (DF) } \\
\text { Experts: Daniel Peres Guimarães (SP), Jerônimo A. Fortunato Jr (PR), } \\
\text { Júlio Faria Honório (SP), Renato Bastos Pope (SC) }\end{array}$ \\
\hline$\underset{\text { PAR }}{\mathbf{E}}$ & $\mathbf{N}_{\text {WPANES }}$ & \\
\hline
\end{tabular}

\section{HANDS EXCLUSIVO PARAPERFUSIONISTAS W 20 DE ABRIL $\mid$ SEXTA-FEIRA | 8h/12:30 I AUDITÓRIO 7}

CIRCUITOS E MONTAGENS EM ECMO

\section{ARENA}

21 DE ABRIL $\mid$ SEXTA-FEIRA $\mid$ 14h/14h50 | AUDITÓRIO 9

HEMOSTASIA EM TERAPIAS DA COAGULAÇÃO:

INSIGHTS EM CIRURGIA CARDÍACA

Palestrante: Renato Arnoni (SP)

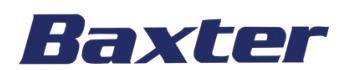




\section{DE ABRIL | QUINTA-FEIRA $12 \mathrm{~h} 30 / 14 \mathrm{~h}$}

\section{AUDITÓRIO 2 \\ ivaNova \\ Health innovation that matters}

ECMO - ABORDAGENS DE CANULAÇÃO - QUAL A MELHOR ESCOLHA?

Moderador: Fernando Antoniali (SP)

Convidados: Andrey Monteiro (RJ)

Beatriz Furlanetto (SP)

Fernando Atik (DF)

Luiz Fernando Caneo (SP)

Temas:

1. ECMO Respiratória: cânulas duplo lúmen e canulação dupla

2. ECMO cardíaca primária: opções de canulação para ECMO VA

3. ECMO pós cardiotomia: truques para boa canulação

4. ECMO Central X Periférica

5. Complicações relacionadas a canulação.

\section{AUDITÓRIO 5}

\section{Eiva:Nova}

PRÓTESE AÓRTICA SEM SUTURAS:

UMA MUDANÇA DE CONCEITO NA ERA TAVI

\section{DE ABRIL | SEXTA-FEIRA $12 \mathrm{~h} 30 / 14 \mathrm{~h}$}

\section{AUDITÓRIO 4}

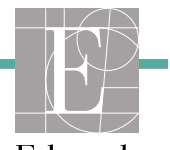

Edwards

ONE EDWARDS VALVE SOLUTION

- Uma discussão sobre Rapid Deployment, TAVI \& Plastia

Moderador: Henrique Murad (RJ)

- Video 3D do implante da válvula de rápida entrega: Intuity

Palestrante: Fabio Jatene (SP)

- O emprego dos anéis na plastia mitral e tricúspide

Palestrante: Francisco Costa (PR)

- Sapien 3 - Desenhando o futuro para TAVI

Palestrante: Alexandre SIciliano (RJ)

\section{AUDITÓRIO 7 \\ contatti medical}

IMPROVEMENTS OF INTRAOPERATIVE SAFETY AND CONVENIENCE BY HTK AND DEL NIDO METHODS

Prof. Claus Preusse (GER)

\section{AUDITÓRIO 8}

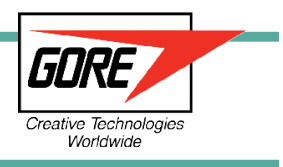

Presidente: Eduardo Keller Saadi (RS)

Moderadores: Eduardo Keller Saadi (RS), Honório Palma (SP), Lucas Barbieri (SP)

- Quando Indicar Tratamento Endovascular na Dissecção do Tipo B não complicada When to Indicate Endovascular Treatment in uncomplicated Type B Dissection

Palestrante: Eduardo Keller Saadi (RS)

- Desenho do Dispositivo Impacta os Resultados Clínicos em Dissecções Tipo B ?

Device Design Impacts Clinical Outcomes in Type B Dissections?

Palestrante: Sarah Leung

Variações da Dissecção Aórtica: Úlcera Penetrante e Hematoma Intramural Variations of Aortic Dissection: Penetrating Ulcer and Intramural Hematoma

Palestrante: Luciano Leitão (PR)

- Tratamento da dissecção tipo A residual Residual treatment in Type A dissection

Palestrante: Gustavo Judas (SP)

- O tratamento da dissecção tipo A pode ser menos paliativa Treatmente of type A dissection may be less palliative

Palestrante: Noedir Antônio G. Stolf (SP) 



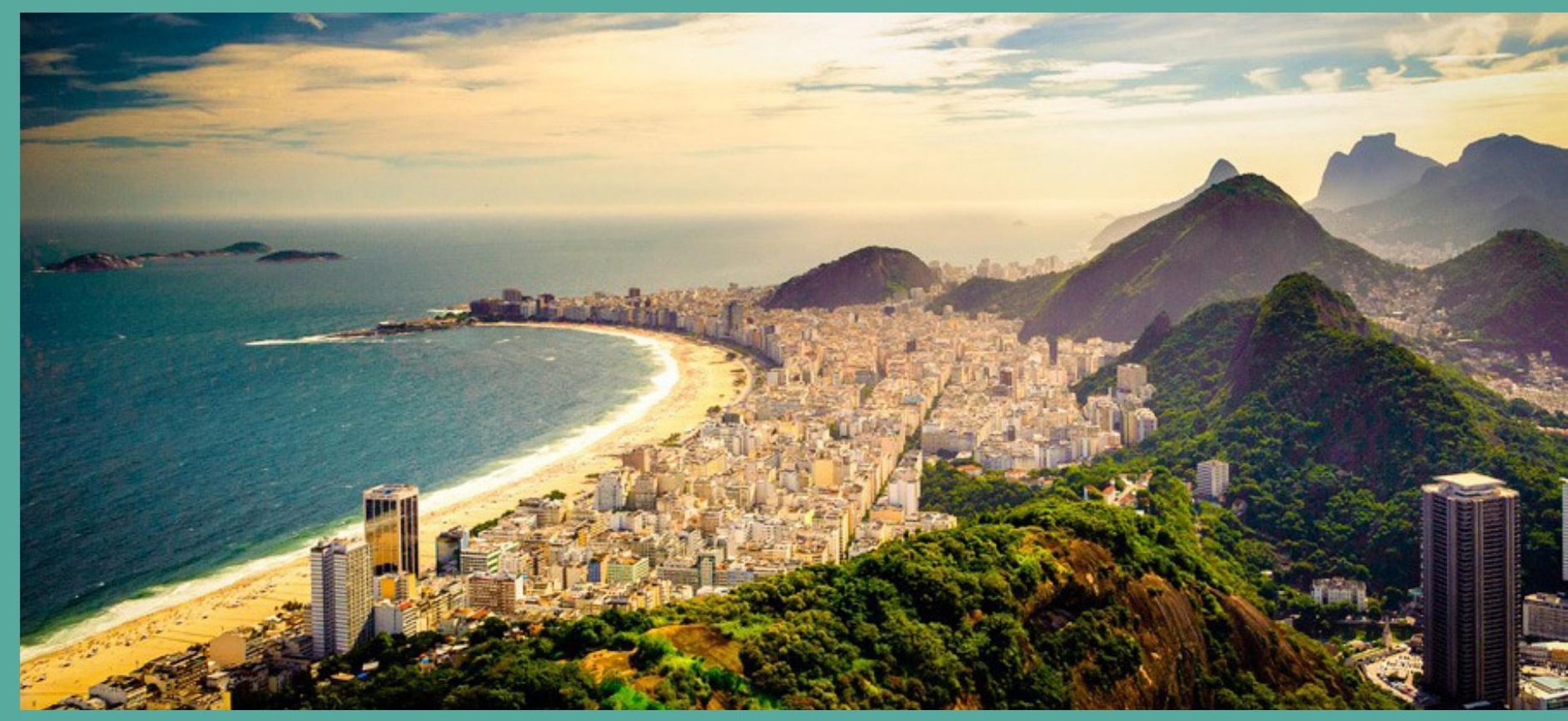

\section{Temas Livies \\ SBCCV}




\section{TL $01 \bullet 8 \mathrm{~h}-8 \mathrm{~h} 15$}

\section{Resultados tardios da reconstrução da via de saída do ventrículo direito com homoenxertos descelularizados em crianças menores de 12 anos}

Leonardo Mulinari, Cristiano Gustavo Hahn, Renato Torres, Eduardo Mendel Balbi Filho, Isabela Hermont, Rafael Torres, Allyson Calixto, Francisco Diniz Affonso da Costa

Introdução: Homoenxertos descelularizados (HD) são considerados uma alternativa promissora, entretanto seus resultados em crianças ainda não são conhecidos. O objetivo deste estudo é relatar nossa experiência de 10 anos com HD na reconstrução da via de saída do ventrículo direito (VSVD) em crianças.

Objetivo: O objetivo deste estudo é relatar nossa experiência de 10 anos com HD na reconstrução da VSVD em crianças.

Métodos: Entre 1996 e 2006, 59 pacientes com mediana de idade de 6,2 anos foram submetidos a reconstrução da VSVD com HD. O tempo de seguimento médio foi de 5,4 anos (0,1-10 anos) e foi completo em 94\% dos casos. Além da avaliação ecocardiográfica, 20 pacientes foram avaliados com angiotomografia. Disfunção estrutural foi considerada quando o gradiente máximo foi $\geq 40$ $\mathrm{mmHg}$ ou quando a insuficiência valvar era $\geq$ moderada.

Resultados: A sobrevida foi de $92 \%$ aos 10 anos e 90\% estiveram livres de reintervenção. O gradiente máximo imediato foi de 13 $\mathrm{mmHg}$ e de $23 \mathrm{mmHg}$ na evolução tardia. Quatro pacientes apresentaram gradiente $\geq 40 \mathrm{mmHg}$ e nove apresentaram insuficiência valvar $\geq$ moderada. Por método atuarial, 85,8\%,75,7\% e 64,9\% dos pacientes estavam livres de estenose, insuficiência ou ambos, respectivamente. A análise univariada demonstrou que idade, diâmetro do enxerto e hipertensão pulmonar foram fatores de risco para a disfunção estrutural. Pela angiotomografia, os enxertos demonstraram ausência ou mínima calcificação tardia.

Conclusão: Os HD tiveram ótimo desempenho hemodinâmico e baixa incidência de disfunção estrutural no grupo pediátrico. Uma característica marcante foi a ausência de calcificação tardia. Embora esses resultados ainda precisem ser confirmados em maior número de pacientes, os HD tiveram resultados superiores aos reportados com outros enxertos em pacientes pediátricos.

\section{TL $02 \bullet 8 \mathrm{~h} 15-8 \mathrm{~h} 30$}

\section{Transplante cardíaco em cardiopatias congênitas: resultados e desafios}

Guilherme Viotto Rodrigues da Silva, Luiz Fernando Caneo, Aída Luiza Ribeiro Turquetto, Leonardo Augusto Miana, Carla Tanamati, Elizio de Souza Rebouças, Maria Raquel Massoti, Janayna Thaina Rabelato, Estela Azeka, Marcelo Biscegli Jatene

Introdução: Estudos recentes demonstram um pior resultado no transplante cardíaco em cardiopatias congênitas (CC), especialmente em pacientes que foram submetidos à cirurgia de Fontan (FT), quando comparados aos portadores de miocardiopatia (MCP).

Objetivo: Avaliar os resultados do transplante cardíaco em CC e compará-los com outras causas não congênitas.

Métodos: No período de janeiro de 1992 a julho de 2016, 184 pacientes foram submetidos a transplante cardíaco pediátrico, sendo divididos em três grupos conforme a etiologia: 52 CC (28,3\%), 11 FT (6\%) e 121 (65,7\%) MCP. Resultados e fatores de risco para mortalidade foram estudados.

Resultados: A necessidade de suporte circulatório mecânico foi maior nos pacientes com MCP. A sobrevida em 30 dias foi de 78,4, 54,5 e 89,3\% para pacientes com CC, FT e MCP respectivamente. A sobrevida em um ano para pacientes com CC, FT e MCP foi de $66,2,54,5$ e $80,8 \%$, respectivamente (log-rank P 0,023). Análise multivariada revelou quatro fatores de risco significativos para mortalidade: tempo de isquemia fria (OR: 1,004, $\mathrm{P}<0,05)$, tempo de circulação extracorpórea (OR: 1,010, $\mathrm{P}<0,001)$, diagnóstico de cardiopatia congênita (OR: 1,961, P=0,006) e suporte circulatório mecânico como ponta para o transplante (OR: 4,725, $\mathrm{P}<0,001)$.

Conclusão: O transplante cardíaco para portadores de cardiopatias congênitas é uma opção aceitável e encorajadora nos pacientes em estágio final de insuficiência cardíaca ou falência da circulação de Fontan. Novas estratégias são necessárias para minimizar os riscos relacionados aos pacientes portadores de cardiopatias congênitas, principalmente aqueles em pós-operatório de cirurgia de Fontan. 


\section{APRESENTAÇÃO DE TEMAS LIVRES - SBCCV}

\section{TL $03 \cdot 8$ Bho-8h45}

\section{Avaliação do pós-condicionamento isquêmico e da atorvastatina na prevenção das lesões de reperfusão. Estudo experimental em ratos}

José Carlos Dorsa Vieira Pontes, Henrique Budib Dorsa Pontes, Euler De Azevedo Neto, Giovanna Serra Da Cruz Vendas, João Victor Cunha Miranda, Leticia Do Espírito Santos Dias, João Victor Durães Gomes Oliva, Murillo Henrique Martins De Almeida, Ian De Oliveira Chaves, Trícia Luna Sampaio, Carlos Henrique Marques Dos Santos, Doroty Mesquita Dourado

Introdução: A lesão de reperfusão pode levar a alterações estruturais e funcionais a nível sistêmico. Nos últimos 20 anos, vários estudos demonstraram que o pós condicionamento isquêmico apresenta eficácia na sua prevenção. Recentemente em alguns estudos as estatinas também demonstraram resultados promissores na proteção contra lesões de reperfusão.

Objetivo: Avaliar a capacidade do pós-condicionamento isquêmico e das estatinas em minimizar lesões de reperfusão à distância, em ratos submetidos à isquemia e reperfusão por clampeamento da artéria aorta abdominal.

Métodos: 41 ratos Wistar Norvégicos foram distribuídos em 5 grupos: Isquemia e reperfusão, Pós-condionamento Isquêmico, Póscondicionamento + estatina, estatina e Sham. Após a eutanásia, os pulmões, fígado, rim e intestino delgado foram submetidos a estudo histopatológico.

Resultados: A média do grau de lesão foi do grupo A: pulmão - 3,6; fígado - 3; intestino - 2; rim - 4. No grupo B: pulmão - 1,6; fígado - 1,5; intestino - 0,66; rim - 2,44. No grupo C: pulmão - 1,2; fígado - 1,2; intestino - 0; rim - 1,22; no grupo D a média no pulmão - 1,2; fígado - 1,2; intestino - 0; rim - 1,11. Os resultados obtidos foram submetidos a tratamento estatístico, aplicando-se o teste de Friedman, sendo estabelecido o nível de significância de $P<0,05$.

Conclusão: Os grupos submetidos ao PoClr, ao pré-tratamento com estatinas e aos dois métodos associados (grupos B, C e D respectivamente) demonstraram menor grau de lesão de isquemia e reperfusão remota em comparação ao grupo submetido a lesão de I/R sem método de proteção.

\section{TL $04 \cdot 8$ 8h45-9h}

\section{A plastia tricúspide associada à intervenção em valva mitral tem impacto?}

Vinicius Fleiria Pimenta, Octavio David Monterroza Humanez, Álvaro Jesus Torres Constante, Diego Gamarra Moreira, Jenny Rivas de Oliveira, Samira Kaissar Ghorayeb, Dorival Julio Della Togna, Magaly Arrais dos Santos, Renato Tambellini Arnoni, Luiz Carlos Bento de Souza

Introdução: Atualmente há evidências de benefício no reparo cirúrgico da valva tricúspide, substituição ou anuloplastia.

Objetivo: Demonstrar o benefício da intervenção na valva tricúspide em pacientes com insuficiência tricúspide moderada ou importante submetidos à cirurgia mitral.

Métodos: Estudo prospectivo de 259 pacientes com doença mitral associada à insuficiência tricúspide moderada a importante em centro especializado, no período de janeiro de 2008 a dezembro de 2015, com a análise de 259 pacientes, sendo 61 do sexo masculino (23,6\%) e 198 do sexo feminino (76,4\%), comparando-se dois grupos: Grupo 1 - 162 pacientes (62,1\%) com idade média 56,42 25,58 anos, que realizaram plastia tricúspide associada à cirurgia mitral, e Grupo 2 - 97 pacientes (37,9\%) com idade média 54,86 $\pm 22,14$, que realizaram cirurgia mitral isolada. Para testar diferenças entre os grupos utilizamos o teste t-Student no caso paramétrico, e na não normalidade utilizamos o teste não paramétrico (teste Mann-Whitney) nas variáveis quantitativas. Nas qualitativas, foi utilizado o teste exato de Fisher para verificar associação com o grupo. Foram considerados significativos $\mathrm{P}<0,05$.

Resultados: Os Grupos 1 e 2 foram pareados. Não houve diferença significativamente estatística entre os grupos na fração de ejeção e classe funcional. Houve melhora da hipertensão pulmonar (40,9\% vs. 21,3\%, P=0,024) e melhor competência da tricúspide $(59,1 \%$ vs. 53,6\%, P=0,019) no Grupo 1.

Conclusão: A intervenção cirúrgica na tricúspide em pacientes com insuficiência moderada a importante, associada à intervenção mitral, mostrou importante benefício. 


\section{TL $05 \cdot 9 h-9 h 15$}

\section{Trombocitopenia após valve-in-valve transcateter}

Renato Cesar de Souza, Leonardo Paim Nicolau da Costa, José Honório Palma da Fonseca, Joaquim Aprígio Nóbrega Batista, Guilherme Viotto Rodrigues da Silva, Lucas Lacerda Araújo, Fabio Biscegli Jatene

Introdução: O "valve-in-valve" (VIV) é uma promessa para o tratamento de pacientes com disfunção de prótese valvar biológica. A trombocitopenia é prevista em procedimentos transcateter e relatada com maior frequência em pacientes submetidos à troca valvar aórtica transcateter. A queda na contagem de plaquetas após procedimentos transcateter está associada a maior mortalidade.

Objetivo: Estudo retrospectivo que objetiva analisar a curva plaquetária dos pacientes submetidos a VIV no InCor-FMUSP e avaliar se há trombocitopenia após este procedimento, sua intensidade, se há diferença no comportamento desta curva entre VIV mitral e tricúspide e sua correlação com mortalidade.

Métodos: Realizada revisão de prontuários de todos os pacientes submetidos a VIV no InCor-FMUSP nos anos de 2015 e 2016. Dos 15 pacientes, 12 foram submetidos a procedimento na valva mitral e 3 na valva tricúspide. A curva plaquetária foi baseada em exames solicitados durante a internação. Os gráficos de médias plaquetárias foram montados até o 60 PO.

Resultados: Em média, os pacientes apresentaram trombocitopenia leve já no $1^{\circ} \mathrm{PO}$ e moderada no $2^{\circ} \mathrm{PO}$. Apenas três pacientes apresentaram trombocitopenia severa nos primeiros 6 dias de pós-operatório. O nadir da curva média foi atingido no 3० PO. Não houve diferença significativa entre as curvas mitral e tricúspide, bem como não houve correlação estatística com mortalidade.

Conclusão: A trombocitopenia é uma realidade no pós-operatório de procedimentos transcateter, e neste estudo não evidenciamos relação com mortalidade.

\section{TL $06 \bullet 9 h 15-9 h 30$}

\section{Tratamento cirúrgico da endocardite infecciosa ativa: preditores de mortalidade em 10 anos de seguimento}

Jenny Lourdes Rivas de Oliveira, Magaly Arrais Dos Santos, Luiz Carlos Bento de Souza, Renato Tambellini Arnoni, Auristela Isabel de Oliveira Ramos, Dorival Julio Della Togna, Samira Kaissar Ghorayeb, Roberto Tadeu Magro Kroll

Introdução: A endocardite infecciosa ativa está associada com elevadas morbidade e mortalidade; a cirurgia é indicada em condições de risco elevado, e os principais determinantes de mortalidade devem ser avaliados.

Objetivo: Identificar os preditores de mortalidade no tratamento cirúrgico da endocardite infecciosa ativa em 10 anos de seguimento, por meio de um estudo observacional prospectivo.

Métodos: Estudo de 88 pacientes diagnosticados com endocardite infecciosa (Duke), com tratamento cirúrgico de janeiro de 2005 a

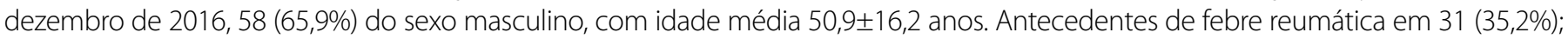
cirurgia cardíaca prévia com implante de prótese valvar em 48 (54,5\%), endocardite de prótese biológica em 45 (93,8\%) e 3 (6,3\%) metálica, 23 (47,9\%) prótese mitral, 20 (41,7\%) aórtica, 5 (10,4\%) próteses mitral e aórtica; e 40 (45,5\%) valva nativa, 19 (47,5\%) valva

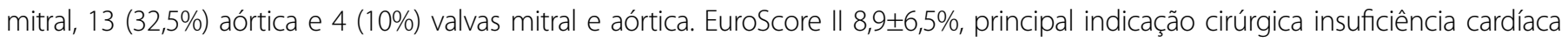
refratária em 38 (43,2\%). Implantadas 68 próteses biológicas (36 aórtica, 32 mitrais), 29 metálicas (12 aórtica, 17 mitrais) e 3 plastias mitrais. Reconstrução do anel aórtico por abscesso com retalho de pericárdio bovino ou tubo protético em 18 (20,5\%). Tempo cirúrgico médio

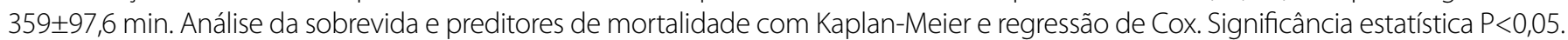

Resultados: Mortalidade global de 20,5\%,15 óbitos (17,05\%) em 30 dias. Principal causa: sepse em 13 (86,7\%); mortalidade tardia em 3 $(3,45 \%)$ por choque cardiogênico. Na análise univariada, os preditores de mortalidade foram hemocultura positiva com microrganismo típico em 48 (54,5\%) P=0,008, mais frequentes Streptococcus viridans 12 (25\%) e Staphylococcus epidermidis 10 (20,83\%); PCR ( $P=0,04 ; H R 1,03$ IC 95\%1,0-1,07); clearance de creatinina ( $P=0,005 ;$ HR0, 98 IC 95\% 0,96-0,99); tempo da cirurgia, a cada 5 minutos, aumento de 1,005 de chance de óbito ( $P=0,03$; HR1,005 IC 95\% 1,001-1,01), a partir de 435 minutos, a cada 5 minutos, aumento de 3,9 de chance de óbito ( $P=0,005$; HR 3,9 IC 95\% 1,51-10,10); idade ( $P=0,001$; HR1, 06 IC 95\% 1,03-1,09); e EuroScore II ( $P=0,003$; HR1, 09 IC 95\% 1,03-1,15).

Conclusão: Hemocultura positiva com microrganismo típico, PCR, clearance de creatinina, idade, EuroScore Il pré-operatórios e o tempo cirúrgico total foram os principais preditores de mortalidade, impactando significativamente na sobrevida em até 10 anos de seguimento. 


\section{APRESENTAÇÃO DE TEMAS LIVRES - SBCCV}

\section{TL $07 \bullet 9 h 30-9 h 45$}

\section{Valve-in-valve mitral sequencial (valve-in-valve-in-valve): o fim de reoperações? Expansão dos limites terapêuticos através de testes hidrodinâmicos in vitro}

Caio Cesar Cardoso, Diego Felipe Gaia dos Santos, José Honório de Almeida Palma da Fonseca, João Roberto Breda, Thiago Cavalcanti Vila Nova de Araújo, Ademir Massarico Braz, Domingo M. Braile, Guilherme Agreli, Celso Ávila, Fabiano André Pereira, Tatiane Lamarca Dias

Introdução: O implante de valva transcateter em bioprótese degenerada (valve-in-valve), em posição mitral, já é terapêutica progressivamente indicada e estabelecida para disfunção de bioprótese, especialmente por estudos demonstrando durabilidade satisfatória das valvas transcateter. Entretanto, com perspectiva futura de degeneração de valvas transcateter, o implante valve-invalve sequencial (valve-in-valve-in-valve) é terapêutica plausível. Estudou-se pouco a alocação de nova valva transcateter em outra previamente implantada, referente à redução de área valvar efetiva (EOA-cm²) e elevação de gradientes transvalvares ( $\triangle \mathrm{P}-\mathrm{mmHg}$ ).

Objetivo: Avaliação hidrodinâmica do implante valve-in-valve-in-valve mitral, delimitando limites terapêuticos.

Métodos: Com Pulse-Duplicator (ViVitro), seguiram-se especificações do FDA para débito cardíaco (5L/min), pressão arterial média (100 $\mathrm{mmHg}$ ) e variação de frequência cardíaca (FC: 70-120 bpm), calculando-se EOA e $\triangle \mathrm{P}$. Confeccionaram-se quatro conjuntos: 1- bioprótese $31 \mathrm{~mm}$ com valvas transcateter 30 mm, 28 mm, 26 mm, 24 mm, 22 mm; 2- bioprótese 29 mm com valvas 28 mm, 26 mm, 24 mm, 22 mm; 3- bioprótese $27 \mathrm{~mm}$ com valvas $26 \mathrm{~mm}, 24 \mathrm{~mm}, 22 \mathrm{~mm}$; 4- bioprótese $25 \mathrm{~mm}$ com valvas $24 \mathrm{~mm}, 22 \mathrm{~mm}$. A cada implante aferiram-se EOA e $\triangle$ P. Replicou-se cada conjunto 10 vezes, repetindo-se os testes em cada conjunto 3 vezes (para cada FC, totalizando 180 medidas).

Resultados: Conjunto 1: valva 30: $E O A=2,18(d p=0,018), \triangle P=3,41(d p=0,045)$; valva 28: $E O A=2,1(d p=0,02), \Delta P=3,6(d p=0,05)$; valva 26: $E O A=1,99(d p=0,01), \triangle P=3,71(d p=0,03)$; valva 24: $E O A=1,67(d p=0,01), \triangle P=5,04(d p=0,06)$; valva 22: $E O A=1,07(d p=0,03)$, $\triangle P=11,42(d p=0,67)$. Conjunto 2: valva 28: $E O A=2,18(d p=0,02), \Delta P=3,61(d p=0,071)$; valva $26: E O A=1,92(d p=0,02), \Delta P=3,71$ $(d p=0,03)$; valva 24: $E O A=1,49(d p=0,01), \triangle P=6,38(d p=0,08)$; valva 22: $E O A=1,1(d p ~ 0,1), \triangle P=11,06$ (dp=0,15). Conjunto 3: valva 26: $E O A=1,63(d p=0,013), \Delta P=5,95(d p=0,11)$; valva 24: $E O A=1,35(d p=0,01), \Delta P=6,98(d p=0,09) ;$ valva $22: E O A=0,85(d p=0,01), \Delta P=17,04$ $(d p=0,38)$. Conjunto 4: valva 24: $E O A=1,085(d p=0,013), \triangle P=11,17(d p=0,25)$; valva 22: $E O A=0,97(d p=0,01), \Delta P=13,46(d p=0,22)$.

Discussão: A utilização de múltiplas valvas transcateter, alternativamente a repetidas cirurgias de substituição valvar, é factível por testes hidrodinâmicos. A valva transcateter possui diâmetro interno e true-id amplos, permitindo tal prática. Independentemente da bioprótese e, consequentemente, da valva transcateter implantada, o implante de valva transcateter sequencialmente menor até 26 mm é possível.

Conclusão: $O$ implante transcateter sequencial valve-in-valve mitral é factível em testes hidrodinâmicos, respeitando-se limites (até valva $26 \mathrm{~mm}$ ) e particularizando-se uso da valva $24 \mathrm{~mm}$.

\section{TL 08 • 9h45-10h}

\section{Reconstrução da raiz da aorta: existe espaço para outra cirurgia além da operação de Tirone?}

Fabrício José de Souza Dinato, Ricardo Ribeiro Dias, José Augusto Duncan, Fábio Fernandes, Félix José Alvarez Ramires, Charles Mady, Fábio Biscegli Jatene

Introdução: A reconstrução da raiz da aorta (RRA) com o tubo valvulado ainda é a cirurgia mais realizada. Discutem-se também as vantagens e desvantagens da bioprótese em relação à prótese mecânica.

Objetivo: Analisar se as operações com o tubo valvulado são capazes de apresentar melhor resultado imediato e tardio que o obtido pela técnica de RRA com preservação da valva aórtica (PVA).

Métodos: No período de janeiro de 2002 a junho de 2016, 448 pacientes foram submetidos à RRA. Estes foram divididos em 3 grupos, de acordo com a técnica de reconstrução: 319 (71,2\%) foram submetidos ao conduto valvado mecânico; 49 (10,9\%) ao tubo valvado biológico e 80 (17,9\%) à técnica de PVA (reimplante). O tempo de seguimento máximo foi de 11 anos.

Resultados: A mortalidade hospitalar foi semelhante entre os grupos. Na análise multivariada observou-se que a idade, a insuficiência renal aguda pré-operatória, a história de cirurgia cardíaca prévia, a necessidade de procedimento associado e o diagnóstico de dissecção tiveram impacto na mortalidade, com o hazard ratio respectivamente de 1,05; 3,35; 2,2; 1,8 e 2,0. Com relação a sobrevida tardia, sobrevida livre de complicações hemorrágicas, tromboembólicas, endocardite e de reoperação, a RRA com PVA foi sempre superior $(\mathrm{P} \leq 0,001)$. Quando comparadas as próteses biológicas e mecânicas, não houve diferença em nenhuma das análises supracitadas.

Conclusão: A cirurgia de PVA deve ser a operação de escolha, sempre que possível, para as operações de RRA. 


\section{TL $09 \bullet 8 \mathrm{~h}-8 \mathrm{~h} 15$}

\section{Seleção de prótese aórtica transcateter por meio de software especializado é mais precisa que o Heart Team}

Álvaro Rösler, Jonathan Fraportti, Gabriel Constantin, Pedro Nectoux, Mauro Pontes, Sílvio César Cazella, Fernando Lucchese

Introdução: O interesse em softwares tem aumentado na área médica. Alguns deles já são utilizados inclusive para diagnóstico. Com a variabilidade das próteses transcateteres, a escolha mais indicada pode se tornar um processo complexo. Esse cenário revela um ambiente propício para a utilização de um software especializado.

Objetivo: Desenvolver um software para auxiliar na seleção da prótese aórtica transcateter mais apropriada.

Métodos: O sistema foi desenvolvido na plataforma Expert Sinta. As regras foram criadas conforme padrões anatômicos indicados pela empresa fabricante. Diâmetro, área e perímetro anulares, bem como os diâmetros da aorta ascendente e dos seios de Valsalva, foram considerados. Após realização de testes de acurácia do sistema, foi aplicado em uma coorte de 22 pacientes submetidos ao implante de CoreValve Medtronic ${ }^{\circledast}$. Posteriormente, as indicações do sistema foram comparadas com as decisões do Heart Team.

Resultados: Em 10 dos 22 pacientes, não houve concordância entre as indicações do sistema e do Heart Team. Os pacientes foram divididos em dois grupos (mesma indicação vs. indicação divergente). As características basais não apresentaram qualquer diferença significativa. Desfechos: mortalidade, AVC, IAM, FA, BAV, regurgitação e leak também não apresentaram diferenças. $O$ gradiente máximo PO foi superior no grupo divergente $-23,9 \mathrm{mmHg}$ vs. $11,9 \mathrm{mmHg}, \mathrm{P}=0,03$, e o gradiente médio apresentou tendência semelhante $-12,6 \mathrm{mmHg}$ vs. 7,7 $\mathrm{mmHg}, \mathrm{P}=0,12$.

Conclusão: $O$ uso do software gerou indicações fidedignas aos parâmetros do fabricante. A escolha do Heart Team pode ser influenciada por critérios subjetivos não considerados pela indústria. Pacientes com divergência na indicação apresentaram gradientes elevados pós-TAVI. Mesmo sem repercussão clínica, esses gradientes podem acarretar disfunção precoce da prótese.

\section{TL $10 \cdot 8$ h $15-8 h 30$}

\section{Resultados da nova prótese transcateter INOVARE Proseal: zero de vazamento perivalvar}

Diego Felipe Gaia dos Santos, Matheus Simonato, Carolina Baeta Neves Duarte Ferreira, João Roberto Breda, Caio Cesar Cardoso, Ademir Massarico Braz, Tiago Vilanova, Fabiano Andre Pereira, Celso Avila, Tatiane Lamarca, José Augusto Marcondes de Souza, Enio Buffolo, José Honório Palma, Luiz Fernando Kubrusly

Introdução: $O$ implante transcateter de válvula aórtica tem surgido como alternativa à troca convencional em pacientes selecionados. Apesar dos resultados, os dispositivos iniciais apresentam limitações, incluindo a presença de vazamento perivalvar residual, contribuindo para um incremento de mortalidade no seguimento tardio.

Objetivo: O objetivo deste estudo é reportar os resultados da nova prótese transcateter Braile Inovare Proseal com um novo mecanismo de redução de vazamento perivalvar.

Métodos: Doze pacientes de alto risco portadores de estenose aórtica grave foram submetidos ao implante de uma nova prótese transcateter dotada de cerdas em sua superfície externa, de maneira a criar um selo antivazamento perivalvar. O STS escore médio foi de 8,5\%. Todos apresentavam estenose valvar aórtica calcificada. O procedimento foi realizado em ambiente cirúrgico híbrido sob controle fluoroscópico e ecocardiográfico. As próteses foram implantadas por via apical sob estimulação ventricular de alta frequência. Controles ecocardiográficos e fluoroscópicos foram realizados.

Resultados: O implante foi possível em todos os casos. Não ocorreu mortalidade operatória, após 30 dias ou durante seguimento. O gradiente transvalvar médio foi reduzido de $44,2 \pm 13,4 \mathrm{mmHg}$ para 6,3 $22,4 \mathrm{mmHg}$. Em 30 dias, apenas 1 paciente apresentou insuficiência perivalvar classificada como discreta; os demais não apresentaram insuficiência. Em 6 meses os resultados ecocardiográficos estavam mantidos. O seguimento variou de 12 a 1 mês. Não ocorreram complicações VARC-2. Tempo máximo de permanência pós implante: 7 dias.

Conclusão: O implante transcateter utilizando a prótese transcateter Braile Inovare Proseal é seguro e capaz de promover redução significativa do vazamento perivalvar, contribuindo para a redução de mortalidade. 


\section{APRESENTAÇÃO DE TEMAS LIVRES - SBCCV}

\section{TL 11• 8h30-8h45}

\section{Implante transcateter "Valve-in-valve" mitral e concomitante TAVI transapical: relato de caso para ilustrar uma mudança de paradigma}

Leonardo Paim Nicolau da Costa, Renato Cesar Souza, Joaquim Aprigio, Lucas Lacerda Araújo, Elinthon Tavares Veronese, Carlos Manuel de Almeida Brandão, Pablo Maria Alberto Pomerantzeff, José Honório Palma da Fonseca, Fabio Biscegli Jatene

Introdução: Os procedimentos valvares transcateteres são uma realidade crescente dentro da especialidade da cirurgia cardiovascular. OTAVI, já bem estabelecido, abriu as portas para outros procedimentos, dentre eles o implante de próteses valve-invalve para o tratamento de biopróteses degeneradas, em pacientes com risco cirúrgico elevado ou proibitivo.

Objetivo: Apresentar o primeiro caso na literatura nacional que realizou, em um mesmo tempo cirúrgico, o implante transcateter por via transapical de uma prótese valve-in-valve em posição mitral, para o tratamento de insuficiência da prótese; e um TAVI para o tratamento da estenose aórtica importante.

Métodos: Trata-se de um paciente masculino, 72 anos, que deu entrada no PS por piora da classe funcional (NYHA IV), diagnosticado com dupla disfunção da prótese mitral com ruptura de folheto e estenose aórtica importante. Possuía 2 cirurgias cardíacas prévias (plástica mitral + RM 1993 e TVM + RM 1998), ponte Sf-DA pérvia, DAC triarterial, IRC não dialítica estágio Illa e FA crônica em uso de ACO. EuroSCORE II 14,77\%. Após discussão com Heart Team, foi optado pelo procedimento combinado transcateter.

Resultados: Procedimento realizado em sala híbrida, guiado por radioscopia e EcoTE, acesso transapical via minitoracotomia anterior esquerda, com implante de 2 próteses Inovare (Braile Biomédica) no 28 em posição aórtica e no 30 valve-in-valve mitral, sem intercorrências, com bom resultado clínico e ecocardiográfico. Paciente recebeu alta hospitalar no 190 PO após término de tratamento antibiótico por BCP.

Conclusão: Com o aumento da sobrevida populacional e consequente prevalência de doenças valvares, opções transcateter têm se mostrado excelentes alternativas, inclusive em procedimentos combinados.

\section{TL $12 \bullet 8 h 45-9 h$}

\section{Resultados tardios da plastia valvar mitral}

Francisco Diniz Affonso da Costa, Daniele de Fátima Fornazari Collatusso, Gustavo Luis dos Santos Martin, Kallynne Carolina Silva Parra, Mariana Cozer Botta, Eduardo Mendel Balbi Filho, Allysson Calixto, Vinicius Nesi Cavichioli

Introdução: A plastia mitral é o procedimento de escolha no tratamento da insuficiência mitral. Quando comparada com a troca valvar, está associada com menor mortalidade imediata e melhor sobrevida tardia.

Objetivo: Revisar os resultados clínicos e ecocardiográficos da plastia mitral em centro de referência com adequado controle ecocardiográfico.

Métodos: Entre 2004 e 2016 foram realizadas 403 plastias mitrais, pelo mesmo grupo cirúrgico. Destas, 108 foram realizadas em uma instituição privada, todas com avaliação ecocardiográfica pré, intra e pós-operatória imediata e tardia. Foram avaliadas mortalidade de 30 dias, sobrevida tardia, classe funcional e necessidade de reintervenções, além dos parâmetros ecocardiográficos

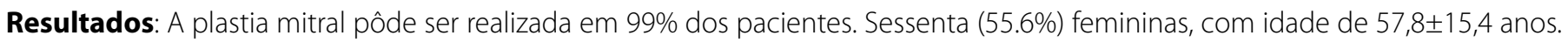
A etiologia mais prevalente foi degeneração mixomatosa/deficiência fibroelástica em 71 (65,7\%). Procedimentos concomitantes foram plastia tricúspide em 13 (12\%), ablação da fibrilação atrial em 14 (13\%) e revascularização do miocárdio em 8 (7,4\%). 0 seguimento clínico médio foi de $47 \pm 40$ (1-142) meses, completo em 94\% dos pacientes, e todos realizaram ecocardiograma tardio de controle. A mortalidade de 30 dias foi 2,8\%. Cinco pacientes foram a óbito na evolução, sendo 4 de causas não cardíacas e 1 de causa cardíaca, o que resultou em uma sobrevida tardia de 80,1\% (LC 95\%=59,8-90) aos 10 anos de evolução. Na evolução tardia, três pacientes apresentaram insuficiência mitral severa, e 2 deles foram submetidos à replastia. Pela curva de Kaplan-Meier, 93\% ( $L C$ 95\%=72,4-98,4) dos pacientes estavam livres de reoperações e 85,1\%(LC 95\%=56,4-94,2) estavam livres de insuficiência mitral severa aos 10 anos.

Conclusão: A plastia mitral, quando realizada em centro de referência, pode ser realizada com baixa mortalidade imediata, boa recuperação funcional e baixa sobrevida tardia, o que se aproxima da população normal pareada para sexo e idade. 


\section{TL 13• 9h-9h15}

\section{Pacientes com dissecção aguda da aorta tipo A apresentando sintomas neurológicos graves: devemos operá-los?}

Ademir Jr. Souto Jacob, Henrique Murad, Rubens Giambroni Filho, Rodrigo Coelho Segalote, André Prado Noronha, Marcio Henrique Cardoso, Edgar Vidotti

Introdução: Acidente vascular encefálico ocorre como uma complicação da dissecção aguda da aorta tipo A em menos de 20\% dos casos e é geralmente considerada uma contraindicação ao tratamento cirúrgico, em razão de seu mau prognóstico demostrado no estudo de Boileau et al.; por outro lado, o tratamento clínico envolve uma alta taxa de mortalidade. Apesar do alto risco envolvido, o tratamento cirúrgico é agora recomendado nestes casos.

Objetivo: Avaliar os resultados de um grupo de pacientes com dissecção aguda da aorta e acidente vascular encefálico submetidos ao tratamento cirúrgico (série de casos).

Métodos: Foram avaliados 5 pacientes com dissecção aguda tipo A com o envolvimento dos vasos do arco aórtico e acidente vascular encefálico submetidos ao tratamento cirúrgico. A equipe cirúrgica avaliou os pacientes desde sua admissão até a alta hospitalar e em visitas ambulatoriais posteriores.

Resultados: Não houve óbito e todos os pacientes submetidos à cirurgia de correção da dissecção obtiveram melhora total ou parcial dos sintomas neurológicos apresentados. Dos 3 pacientes que se encontravam em coma, todos saíram do estado comatoso; 2 ficaram completamente assintomáticos e 1 com sequela leve de afasia e hemiplegia.

Conclusão: Comprometimento neurológico agudo, incluindo o coma, na dissecção da aorta ascendente, não deve ser considerado uma contraindicação ao tratamento cirúrgico.

\section{TL $14 \cdot 9 h 15-9 h 30$}

\section{A ablação bipolar é superior à ablação unipolar no tratamento cirúrgico da fibrilação atrial}

Leonardo Secchin Canale, Cristiane Lamas, Bruno Azevedo, Erica de Abreu Macedo, Cristiane Lamas, Bruno Azevedo, Roberta de Lima Machado, Cintia Cristina Chaves Mattoso

Introdução: A fibrilação atrial é arritmia frequentemente encontrada em pacientes a serem submetidos a cirurgia valvar mitral. A ablação concomitante dos átrios é opção de tratamento para essa arritmia.

Objetivo: Avaliar a eficácia, em termos de conversão ao ritmo sinusal, da ablação combinada da fibrilação atrial em pacientes submetidos a cirurgia valvar mitral. Comparar as formas de energia unipolar e bipolar utilizadas na ablação.

Métodos: Por meio de acompanhamento ambulatorial com ECG e Holter 24 horas, os pacientes submetidos à cirurgia valvar e ablação foram avaliados para definir ritmo cardíaco e falha terapêutica. Por meio da análise univariada de características do préoperatório, foram investigados fatores de risco para permanência da arritmia.

Resultados: Noventa e nove pacientes, sendo 76\% mulheres, com idade média de 50,9 anos (DP: 11,2 anos) foram submetidos a cirurgia valvar mitral e ablação unipolar (25 pacientes) ou bipolar (74 pacientes) entre janeiro de 2008 e julho de 2012. A maioria dos pacientes (74\%) apresentava etiologia reumática para lesão mitral. A mortalidade cirúrgica foi de 7\%. Após 4 anos, $67 \%$ dos pacientes submetidos a ablação bipolar estavam em ritmo sinusal versus nenhum paciente no grupo unipolar $(P<0,001)$. Dentre as variáveis pré-operatórias investigadas, a presença de FA contínua (vs. paroxística) e o tempo de FA prévio à cirurgia foram marcadores de falha terapêutica.

Conclusão: A ablação atrial concomitante à cirurgia mitral realizada com energia bipolar oferece resultados superiores à energia monopolar após 4 anos (67 vs. 0\%). FA contínua e tempo de FA prévio à cirurgia são fatores de risco para falha terapêutica. 


\section{APRESENTAÇÃO DE TEMAS LIVRES - SBCCV}

\section{TL $15 \cdot 9 \mathrm{~h} 30-9 \mathrm{~h} 45$}

\section{Análise das resistências elétricas em eletrodos de marca-passo no período 2010-2016 em hospital de Belo Horizonte: um estudo de coorte não concorrente}

Isabela Tiveron Almeida, Paula lara de Sousa, Eduardo Augusto Victor Rocha, Wendell Alves Horta, Edson Viana Junior

Introdução: O uso de eletrodos eluídos em corticosteroide, iniciado na década de 1980, contribui para a redução da resistência elétrica crônica.

Objetivo: Comparar a resistência elétrica no perioperatório e após 6 meses do implante de marca-passo.

Métodos: 77 pacientes submetidos a implante de marca-passo em instituição única. As resistências elétricas foram comparadas no momento do implante e com seis meses de pós-operatório.

Resultados: A resistência elétrica atrial perioperatória foi de $575 \Omega$ e crônica de $361,25 \Omega$, com valor de $P<0,0001$. A resistência elétrica ventricular perioperatória foi de 776,25 $\Omega$ e crônica de $529 \Omega$, com $\mathrm{P}<0,0001$.

Discussão: $O$ implante de eletrodos de marca-passo desencadeia processos inflamatórios e formação de cápsulas fibrosas ao redor dos eletrodos, resultando em aumento da resistência elétrica. Os corticosteroides agem reduzindo esta inflamação local, contribuindo para a redução da resistência elétrica crônica, conforme constatado em nosso estudo.

Conclusão: Os eletrodos eluídos em corticosteroide apresentam redução da resistência elétrica ao longo do tempo.

\section{TL $16 \cdot 9 h 45-10 h$}

\section{Incidência de estenose carotídea eacidente vascular encefálico isquêmico em pacientes submetidos a cirurgia cardíaca não coronariana}

João Paulo Nadal, Mário Augusto Cray da Costa, Marcelo Derbli Schafranski

Introdução: $O$ acidente vascular encefálico é uma complicação que ocorre em 2\% das cirurgias cardíacas e permanece como uma das mais importantes causas de mortalidade e morbidade em pós-operatório.

Objetivo: Avaliar: a) a incidência de AVE em cirurgia não coronariana; b) a incidência de lesões de carótidas nesses pacientes; c) os fatores de risco associados com estenose carotídea; d) os fatores de risco associados à ocorrência de AVE nos pacientes submetidos à cirurgia cardíaca não coronariana.

Métodos: Foram avaliados os dados de 211 pacientes, que foram atendidos pelo serviço de cirurgia cardíaca entre 2009 e 2016 , na Santa Casa de Misericórdia de Ponta Grossa. Para a comparação de médias foi utilizado o teste T de Student, e para comparação de variáveis categóricas, o teste qui-quadrado bicaudado com correção de Yates e regressão logística. Para variáveis com valores menores que 5 foi utilizado, para comparação, o teste de Fisher.

Resultados: Após o ajuste, por regressão logística, permaneceram significativas apenas: estenose carotídea (OR: 6,25; P=0,014), hipertensão pulmonar moderada e severa (OR: 12,5); (OR: 8,95); DPOC (OR: 4,73; P=0,028) e arteriopatia extracardíaca (OR: 21,33; $P=0,0001)$.

Discussão: Os resultados aqui encontrados sugerem que as lesões carotídeas estão associadas ao AVE, porém não afirmam que são sua etiologia propriamente dita, deixando ainda controverso o real benefício do tratamento profilático das lesões carotídeas nos pacientes sem sintomas neurológicos.

Conclusão: A incidência de AVEi foi de 4,73\%, ao passo que a incidência de estenose carotídea igual ou acima de 50\% foi de 7,59\%. Os fatores de risco significativos para AVEi perioperatório em cirurgia cardíaca foram estenose carotídea igual ou maior que 50\% e hipertensão pulmonar moderada e severa. 


\section{TL 17・ $10 \mathrm{~h} 45-11 \mathrm{~h}$}

\section{Resultados em longo prazo da cirurgia de Jatene}

Camila Lino Martins Rodrigues da Silva, Daniel Alves Mascarenhas, Maira Cerqueira Weigel, Elaine Cristina Furquim, Rogério Souza, Patricia Figueiredo Elias, leda Biscegli Jatene, Marcelo Biscegli Jatene

Introdução: A transposição das grandes artérias (TGA) é a cardiopatia congênita cianogênica mais prevalente no período neonatal. Apresenta evolução totalmente desfavorável caso não seja corrigida cirurgicamente, sendo a cirurgia de Jatene a escolha para o tratamento.

Objetivo: Descrever características clinicas e funcionais dos pacientes submetidos à cirurgia de Jatene em seguimento ambulatorial de longo prazo.

Métodos: Estudo retrospectivo de pacientes submetidos à cirurgia de Jatene, em acompanhamento ambulatorial. As seguintes variáveis foram avaliadas: tipo de TGA, realização da manobra de Lecompte, desenvolvimento de arritmias, necessidade de marcapasso, presença de insuficiência cardíaca (IC), insuficiência ou estenose das grandes artérias e comunicação interventricular (CIV) residual.

Resultados: Foram incluídos 113 pacientes, 73 (64,6\%) do sexo masculino, com tempo médio de follow-up de 9,8土8,7 anos. A média da idade na cirurgia foi de 2,8 $\pm 8,4$ meses. Dos pacientes em que foi realizada a manobra de Lecompte, $22 \%$ evoluíram com estenose da neopulmonar em comparação com $47 \%$ dos que não foram submetidos à manobra $(P=0,033)$. Necessidade de implante de marca-passo definitivo ocorreu em 8,3\% dos pacientes, IC em 10,7\%, dilatação da neoaorta em 49,1\%, estenoses da neopulmonar em 30\% e da neoaorta em 10,1\% e CIV residual em 8,1\% dos pacientes.

Conclusão: No seguimento de longo prazo pós-cirurgia de Jatene para correção anatômica de TGA, a incidência de estenose da neopulmonar foi menor nos pacientes em que foi realizada manobra de Lecompte. Aproximadamente metade dos pacientes evoluiu com dilatação da neoaorta e uma pequena parcela com necessidade de implante de marca-passo definitivo, CIV residual ou insuficiência cardíaca.

\section{TL $18 \bullet 11 \mathrm{~h}-11 \mathrm{~h} 15$}

\section{Cirurgia cardíaca minimamente invasiva - experiência inicial no tratamento de lesões valvares}

Daniel Peres Guimarães, Olivio Souza Neto, Anderson Nascimento, Eduardo Parrilho, Giani Hirose, Thiago Ferreira, Rui M. S. Almeida

Introdução: A cirurgia minimamente invasiva tem se mostrado uma realidade em centros de excelência para o tratamento de lesões valvulares. Há ainda um grande debate no tocante a tempos de clampeamento aórtico, CEC, assistência ventilatória, internação em UTI e hospital.

Objetivo: Apresentar a experiência inicial no tratamento de lesões valvulares em um único serviço, por meio de técnicas minimamente invasivas e vídeo-assistidas.

Métodos: No período de agosto a novembro de 2016, analisaram-se, prospectivamente, dados de 12 pacientes, de pré, trans e pós-operatórios, operados por técnicas minimamente invasivas/vídeo-assistidas, com lesões valvulares.

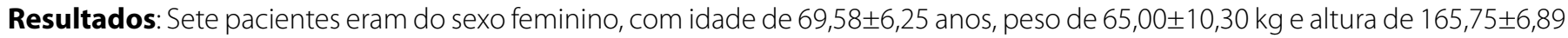
$\mathrm{cm}$. A classe funcional era II (58,33\%) e III no restante. O EuroScore logístico foi de 4,81 $\pm 6,28$. Cinco pacientes apresentavam IM, cinco EAo e dois, EM. Em 11 foram realizadas trocas por próteses biológicas (número médio 28,33 mitral e 22,60 aórtico) e uma plastia.

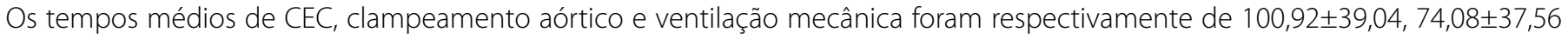

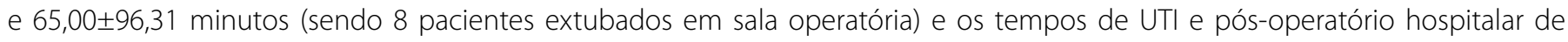

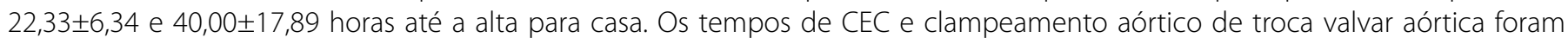
menores que os da mitral (121,50/60,40 e 93,17/35,40 min).

Conclusão: $O$ tratamento de lesões valvares por técnicas minimamente invasivas mostrou ser um método seguro e com boa evolução clínica, minimizando de forma importante os tempos de ventilação mecânica, internação em UTI e hospital. 


\section{APRESENTAÇÃO DE TEMAS LIVRES - SBCCV}

\section{TL 19・11h15-11h30}

\section{Uso de nanoemulsões lipídicas como veículos de paclitaxel e de metotrexato no tratamento da doença vascular do coração transplantado em coelhos}

Lucas Regatieri Barbieri, Domingos Lourenco Filho, Elaine R Tavares, Paulo S Guitierrez, Raul C. Maranhão, Noedir A. G. Stolf

Introdução: A doença vascular do coração transplantado consiste em um processo inflamatório proliferativo que compromete o sucesso a longo prazo do transplante cardíaco e não há prevenção ou tratamentos efetivos. Uma nanoemulsão lipídica (LDE) pode carregar agentes quimioterápicos na circulação e concentrá-los nos enxertos cardíacos dos coelhos.

Objetivo: Investigar os efeitos tóxico e redutor da doença do vascular do coração transplantado em coelhos.

Métodos: Vinte e oito coelhos alimentados com dieta com teor de 0,5\% de colesterol e submetidos a transplante cardíaco herotópico, tratados com ciclosporina e alocados em 4 grupos de 7 animais. Um grupo recebeu a associação de metotrexato e LDE endovenosa; o segundo grupo, paclitaxel e LDE; o terceiro, metotrexato e paclitaxel; e o grupo controle, solução salina intravenosa. Os animais foram sacrificados 6 semanas após o procedimento.

Resultados: O grupo paclitaxel associado ao LDE apresentou redução em 50\% de estenose em artérias coronárias. Já nos grupos que usaram metotrexato associado a LDE ou paclitaxel combinado com metotrexato e associado a LDE, houve redução em 18\% da estenose coronariana em relação ao grupo controle. Nos três grupos tratados, houve redução do infiltrado macrofágico.

Discussão: No grupo que recebeu metotrexato associado a LDE, a expressão gênica de fatores pró-inflamatórios foi reduzida drasticamente, enquanto a expressão de agentes anti-inflamatórios (p. ex., IL-10) aumentou.

Conclusão: Não houve toxicidade. A associação paclitaxel e LDE promoveu melhora importante na vasculopatia dos enxertos. As associações metotrexato e LDE e metotrexato mais palcitaxel e LDE reduziram a estenose de coronárias, porém sem significância estatística. Tais resultados podem servir de ponte para novos ensaios clínicos.

\section{TL $20 \bullet 11 \mathrm{~h} 30-11 \mathrm{~h} 45$}

\section{Prevalence and clinical outcomes of hyperglycemia in the perioperative period of cardiac surgery according to diabetes status}

Mauro Ricardo Nunes Pontes, Álvaro Rösler, Jonathan Fraportti, Gabriel Constantin, Pedro Nectoux, Isabella Garcia Paiva, Tainá Silva Carvalho, José Dario Frota Filho, Marcela da Cunha Sales, Eraldo Lúcio de Azevedo, Fernando Lucchese

Introduction: The impact and management of perioperative hyperglycemia in cardiac surgery is controversial.

Objective: Evaluate the prevalence and clinical impact of non-insulin-dependent DM and insulin-dependent DM in cardiac surgery patients.

Methods: Cohort study with 2020 patients submitted to CABG. Glucose was measured at $6 \mathrm{~h}$ intervals in the first day, and at $12 \mathrm{~h}$ intervals up to $48 \mathrm{hs}$ postoperatively. Outcomes included hospital length of stay (LOS), cardiopulmonary complications, major infections and death. Maximum PO glucose measurement was used to define glycemic control - good $<180 \mathrm{mg} / \mathrm{dL}$ or poor $>180$ $\mathrm{mg} / \mathrm{dL}$.

Results: 69.2\% male, 64 10 years. Non-DM: 1263 (62.5\%); DM: 757 (37.5\%). 499 (24.7\%) are NIDDM, and 258 patients (12.7\%) are IDDM. IDDM patients are younger, female, with higher risk scores, had more previous MI, CKD and hemodialysis. DM patients showed worst PO glucose control, especially in IDDM patients. DM in general and IDDM did not increased death and MACCE. DM and IDDM increased sternal infection by 54\% ( $P=0.005)$ and $110 \%(P<0.001)$, major infections $70 \%(P=0.035)$ and $100 \%(P<0.001)$, and length of stay (LOS) in 1 day ( $P<0.01$ both). In non-DM patients, poor PO glycemic control was associated with death and MACCE, which did not occur with NIDDM and IDDM patients. The post operatory glucose was an independent predictor of death after CABG. For each $10 \mathrm{mg} / \mathrm{dL}$ increase in PO glucose, death rate increase 9\%, OR 1.009, $\mathrm{P}<0.001$.

Conclusion: $37.5 \%$ of the patients submitted to CABG had DM. These patients had $70-100 \%$ more sternal and major infections and higher LOS. In non-DM patients, worst PO glucose control is associated with higher mortality and MACCE. 


\section{TL $21 \bullet 11 \mathrm{~h} 45-12 \mathrm{~h}$}

\section{Impacto da avaliação renal na morbidade e mortalidade após cirurgia cardíaca: creatinina sérica é suficiente?}

Camila Perez de Souza Arthur, Omar Asdrúbal Vilca Mejia, Diogo Ostemack, Marcelo Arruda Nakazone, Maxim Goncharov, Luiz Augusto Ferreira Lisboa, Luís Alberto Oliveira Dallan, Pablo M. A. Pomerantzeff, Fabio Biscegli Jatene, Grupo de Estudo REPLICCAR

Introdução: O nível de função renal basal antes da cirurgia cardíaca influencia no risco de morbidade e mortalidade pós-operatórias. Portanto, a avaliação adequada da função renal é prioritária.

Objetivo Avaliar se a creatinina sérica (CS) é suficiente ou se o clearance de creatinina (CC) é necessário para estratificar pacientes submetidos a cirurgia cardíaca.

Métodos: Foram estudados 3.285 pacientes incluídos de forma prospectiva, consecutiva e mandatória no REPLICCAR. Valores de CS, CC (Cockcroft-Gault), EuroScore II e de outras 60 variáveis pré-operatórias foram analisadas para morbidade e mortalidade hospitalares. O impacto das variáveis foi avaliado mediante testes de calibração e discriminação. Por meio da regressão logística múltipla foram criados modelos multivariados (MM) independentes com CS e com CC para predição de risco de morbimortalidade após cirurgia cardíaca.

Resultados: A CS esteve associada com morbidade e mortalidade, mas não calibrou adequadamente os grupos de risco. 0 CC, além de se associar com morbidade e mortalidade, calibrou satisfatoriamente. A CS fica descalibrada a partir de 1,35 mg/dL $(P<0,001)$ e subestima em duas vezes com valores $\geq 1,60 \mathrm{mg} / \mathrm{dL}$. A curva ROC revelou que o CC é superior à CS na discriminação de morbidade e mortalidade. No MM sem o CC, a CS foi preditora unicamente de morbidade; já no MM sem a CS, o CC foi preditor de mortalidade e o único preditor de morbidade.

Conclusão: O CC é superior à CS na estratificação de risco dos pacientes submetidos a cirurgia cardíaca. Ressaltamos que, para valores de CS $\geq 1,60$, o cálculo do CC é necessário no consentimento livre e esclarecido e no planejamento perioperatório.

\section{TL $22 \bullet 12 \mathrm{~h}-12 \mathrm{~h} 15$}

\section{Emprego da ultrassonografia com Doppler para mapeamento de veia safena na obtenção de enxertos para cirurgia de revascularização do miocárdio}

Fillipe Campos Lopes, Oscar Willian Bomfim, Diego Gamarra Moreira, Magaly Arrais dos Santos, Luiz Carlos Bento de Souza, Paulo Chaccur, Caio Bottini Cruz, Getulio Lubanco Filho, Jenny Rivas de Oliveira

Introdução: A veia safena magna é utilizada como enxerto em cirurgias de revascularização do miocárdio, e as complicações da dissecção podem ser minimizadas com o uso da ultrassonografia para mapeamento venoso e marcação do sítio de dissecção.

Objetivo: Analisar, por ensaio clínico, o emprego da ultrassonografia vascular para mapeamento da veia safena na cirurgia de revascularização do miocárdio para definir viabilidade e sítio da dissecção.

Métodos: Selecionamos para esse estudo prospectivo 151 pacientes consecutivos submetidos à cirurgia de revascularização do miocárdio com o uso da veia safena magna, divididos em dois grupos: Grupo 1- 84 pacientes com mapeamento e marcação da veia safena magna com ultrassonografia; Grupo 2- 67 pacientes com veia safena magna dissecada sem estudo prévio. Os grupos foram pareados, com seguimento no 10, $5^{\circ}$ e $30^{\circ}$ dia de pós-operatório. Os desfechos primários foram incisão da perna contralateral e complicações da ferida operatória em até 30 dias.

Resultados: Houve necessidade de abertura das duas pernas em 6 (3,9\%) pacientes do grupo 2 (RR 2,37, 1,96-2,87, IC 95\%, P 0,007). Em até 30 dias ocorreram complicações da ferida operatória em 33 (23,4\%) pacientes, 21 (13,9\%) no Grupo 2 e 12 (7,9\%) no Grupo 1 (RR 3,09, 1,37-6,94, IC 95\%, P 0,003). Em até 30 dias ocorreram 4 (2,8\%) óbitos, todos no Grupo 2 (RR 2,347, 1,94-2,84, IC 95\%, P 0,036).

Conclusão: O emprego da ultrassonografia vascular para mapeamento da veia safena magna em cirurgia de revascularização miocárdica identificou e avaliou adequadamente a veia safena, reduzindo significativamente as complicações cirúrgicas e incisões desnecessárias. O método não invasivo é de fácil aplicação e deveria ser utilizado de rotina. 


\section{APRESENTAÇÃO DE TEMAS LIVRES - SBCCV}

TL $23 \cdot 12 \mathrm{~h} 15-12 \mathrm{~h} 30$

\section{Biopróteses de liberação rápida: uma mudança de paradigma. Experiência do ICP}

Euclides Martins Tenorio, Fernando Moraes Neto, Cibelle Padilha, Igor C. Silva, Fabiana Oliveira, Carlos Roberto Moraes, Manuella Varejão, Igor Paiva

Introdução: A evolução natural da doença valvar aórtica leva a um comprometimento importante da função ventricular esquerda. O aumento de morbidade mortalidade nos portadores dessa doença leva a um desafio a mais para uma opção terapêutica cirúrgica de sucesso, com baixo índice de complicações e melhora dos resultados, o que se tem observado na utilização das biopróteses de liberação rápida.

Objetivo: Aplicação cirúrgica das biopróteses de liberação rápida na substituição da valva aórtica.

Métodos: No período de janeiro a outubro de 2016, no ICP-PE, foram operados 12 pacientes com doença valvar aórtica, nos quais foram implantadas biopróteses de liberação rápida. Dez destas biopróteses eram do modelo Perceval e duas Intuity. Seis pacientes eram do sexo masculino e seis, do sexo feminino. A idade variou de 49 a 81 anos, com média de 64 anos. Observou-se a morbidade e a mortalidade do grupo, bem como aspectos da técnica cirúrgica do implante.

Resultados: Técnica cirúrgica de fácil reprodutividade. Neste grupo houve 3 procedimentos combinados com cirurgia de revascularização do miocárdio (25\%). Como complicação principal foi observado BAVT em um caso (8,3\%). Não houve mortalidade relacionada diretamente à técnica cirúrgica, entretanto uma paciente (8,3\%) foi a óbito decorrente de sepse. O gradiente transprotético pós-operatório foi praticamente inexistente.

Conclusão: A utilização das biopróteses de liberação rápida mostrou-se uma técnica de fácil aplicabilidade e reprodutividade, diminuindo significativamente o tempo de clampeamento aórtico e, desta forma, contribuindo para uma diminuição dw morbidade e mortalidade no grupo de pacientes portadores de doença degenerativa da valva aórtica.

\section{TL $24 \cdot 12 \mathrm{~h} 30-12 \mathrm{~h} 45$}

\section{Hemotransfusão em cirurgia cardíaca e sua relação com desfechos adversos em pós-operatório precoce}

Ana Paula Tagliari, Anderson Castro de Souza, Lucas Molinari Veloso da Silveira, Marcelo Curcio Gib, Tanara Martins de Freitas, Cristiano Blaya Martins, Leandro Totti Cavazzola, Orlando Carlos Belmonte Wender

Introdução: Hemodiluição, sangramento transoperatório e utilização de circulação extracorpórea (CEC) são algumas das variáveis que mantêm as taxas de transfusão de hemoderivados em cirurgia cardíaca persistentemente elevadas.

Objetivo: Analisar a frequência de hemotransfusão em cirurgia cardíaca e o impacto desta na incidência de desfechos clínicos pós-operatórios.

Métodos: Coorte, prospectiva, unicêntrica, de maio de 2015 a outubro de 2016, em um hospital universitário. Dados foram analisados em SPSS 18.0, variáveis contínuas expressas como média e desvio padrão (DP) e categóricas como porcentagem. Testes X2, regressão logística uni e multivariada foram utilizados quando aplicados.

Resultados: Dos 199 pacientes avaliados 72 (36,2\%) necessitaram de hemotransfusão no trans (32,7\%) e/ou pós-operatório $(15,2 \%)$. Variáveis preditoras em análise univariada foram: valores pré-operatórios de tempo de tromboplastina parcial ativada (TTPa) $(P=0,002)$, índice internacional normatizado (INR) $(P=0,011)$, hematócrito $<28 \%(P<0,001)$, doença renal crônica (DRC) $(P=0,034)$, cirurgia de urgência ou emergência (U/E) $(P=0,002)$, tempos de $C E C(P<0,001)$ e isquemia $(P<0,001)$ e de sangramento transoperatório $(P<0,001)$. Em análise multivariada mantiveram-se preditores: TTPa (RR 1,1 IC 95\% 1,0-1,2; $P=0,031$ ) e sangramento transoperatório (RR 1,0 IC 95\% 1,0-1,01; $P<0,001$ ). Sexo masculino mostrou-se protetor (RR 0,35 IC 95\% 0,14-0,9; $P=0,025$ ). Necessidade de hemotransfusão associou-se à ventilação mecânica prolongada (> 24h) (2,4 vs. 28,6\%), broncopneumonia (2,4 vs. 18,6\%), IRA (6,3 vs. 28,6\%), DRC exacerbada (0,8 vs. 12,9\%) e óbito em pós-operatório precoce (1,6 vs. 18,6\%).

Conclusão: Sexo feminino, TTPa, cirurgia em caráter U/E e sangramento transoperatório mostraram-se preditores de necessidade de transfusão, a qual esteve associada a aumento no risco de eventos adversos pós-operatórios. 



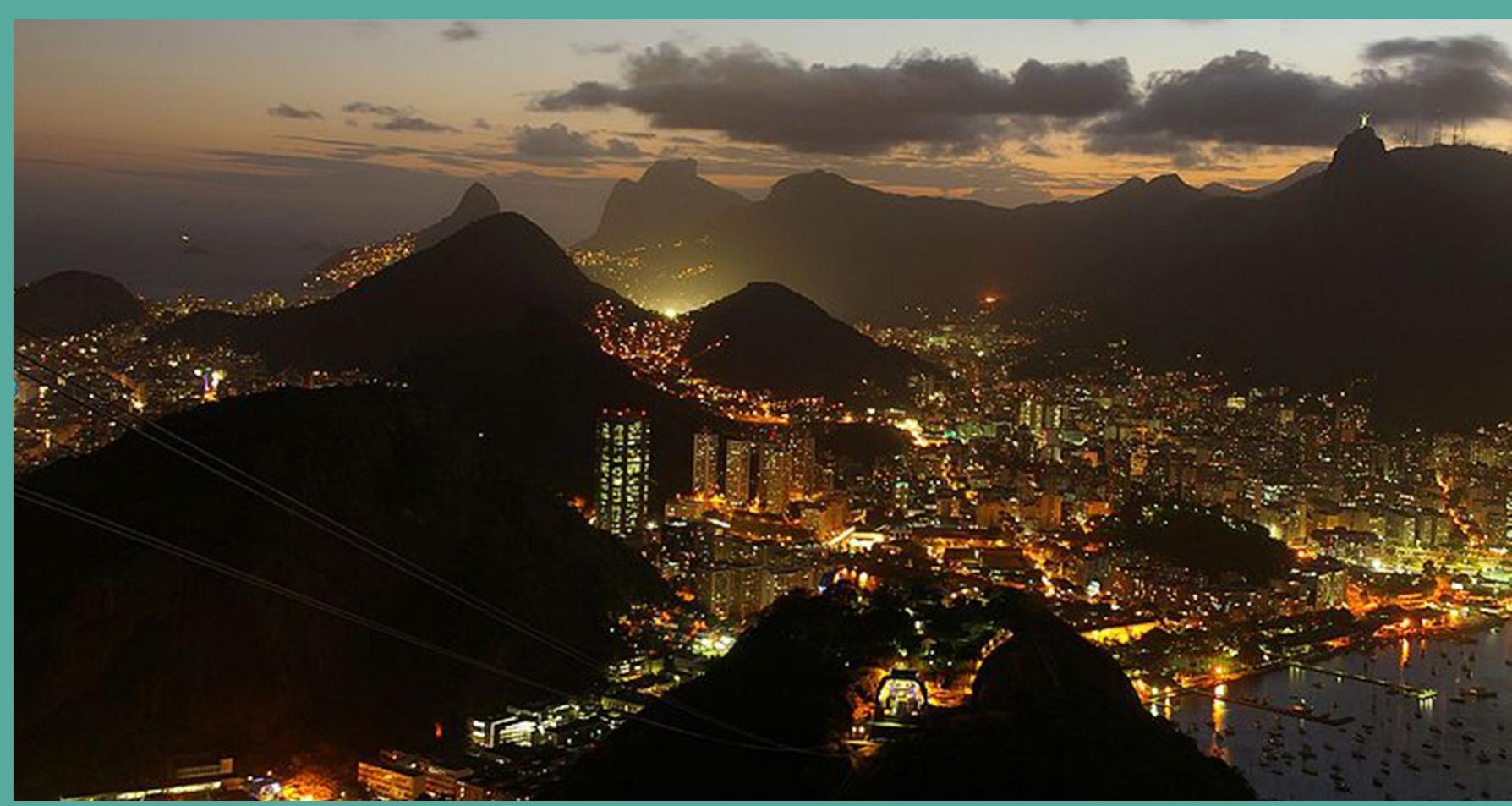

\section{Pôsteres SBCCV}




\title{
PO $01 \cdot 10 h 05-10 h 10$
}

\section{Simulação realística como método de ensino e treinamento de novas técnicas de implante de stent válvula cardíaca por cateter}

\author{
Miguel Angel Maluf, Tatiana Guimarães e Santos, Gabriela Santoro, Caio Sobrinho, Mauricio L. Vaz
}

Introdução: O implante de um stent válvula por cateter representa o mais significativo avanço na cirurgia das válvulas cardíacas. O domínio da técnica de implante requere treinamento do profissional, para minimizar as intercorrências e evitar a reversão de um procedimento, pouco invasivo para uma cirurgia convencional.

Objetivo: Utilizar a simulação realística como método de ensino e treinamento para abordagem e implante de stent válvula cardíaca por cateter.

Métodos: Vinte corações suínos foram utilizados para treinamento de 11 diferentes vias de abordagem e 55 implantes de stent válvula expansível de poliuretano. Os procedimentos realizados foram: 1- valva pulmonar: transventricular, transfemoral; 2- valva aórtica: transapical, transfemoral, transaórtica, transarterial (a. subclávia); 3-valva mitral: transapical, transfemoral, transatrial; 4- valva tricúspide: transfemoral, transatrial; 5-rResgate de próteses embolizadas. O score para classificados foi 0 a 50 pontos.

Resultados: A curva de aprendizado permitiu um significativo progresso da habilidade técnica, segurança e melhora do score, alcançando uma média de 45,5.

Discussão: Novas técnicas de abordagem em cirurgia cardiovascular requerem treinamento e preparo para sua execução naformação de especialistas. Motivação e oportunidades serão requisitos necessários para a habilitação profissional, independentemente do grau de formação.

Conclusão: A simulação realística é um método consagrado para o ensino e o treinamento de habilidade em cirurgia e permite o aprendizado de novas técnicas que serão aplicadas na cirurgia experimental ou na prática clínica, com o intuito de melhorar os resultados e minimizar as intercorrências.

\section{PO 02 - 10h10-10h15}

\section{Avaliação do remodelamento ventricular nas operações de reconstrução da raiz da aorta e preservação valvar}

Marcelo Werneck Salles De Carvalho, Ricardo Ribeiro Dias, Fabrício José de Souza Dinato, José Augusto Duncan, Fábio Fernandes, Félix José Alvarez Ramires, Charles Mady, Fábio Biscegli Jatene

Introdução: O remodelamento ventricular reverso (RVR) é fenômeno conhecido no tratamento cirúrgico da valvopatia aórtica.

Objetivo: Verificar se o grau de insuficiência aórtica (IAo) ou a classe funcional (CF) da insuficiência cardíaca interferem no RVR e na sobrevida dos pacientes submetidos à reconstrução da raiz da aorta (RRA) com preservação da valva aórtica (PVA).

Métodos: No período de maio de 2005 a junho de 2016 foram realizadas 80 RRA com PVA através da técnica do reimplante. Foram avaliados quanto ao grau de IAo (69\% IAo moderada/importante) e a CF (9\% CF III/IV). Para a análise do remodelamento ventricular esquerdo consideraram-se volumes e diâmetros (VDFVE, VSFVE, DDFVE, DSFVE), tamanho do átrio esquerdo (AE), índice de massa (IM) e fração de ejeção (FE).

Resultados: A mortalidade hospitalar e tardia foi semelhante nos dois grupos. Durante o tempo de seguimento de até 10,2 anos e seguimento ecocardiográfico de até 5,5 anos (85\% dos pacientes), observou-se que, para IAo moderada-importante, houve maior redução ventricular do diâmetro, volume, IM e tamanho do AE. Quando se analisaram os pacientes em CF III e IV comparados com CF I e II, e o subgrupo em que se realizou plastia da valva aórtica, observou-se o mesmo benefício de RVR, à exceção da redução do tamanho do $A E$.

Conclusão: $O$ RVR foi significativamente maior quando a IAo era de maior grau, porém também se observou significativo remodelamento reverso quando a IAo era menor que moderada. 


\section{PO 03 • 10h15-10h20}

\section{Stent válvula expansível depoliuretano. Resultado detestesfísicos, hidrodinâmicoseexperimentais}

Miguel Angel Maluf, Mariana Massei Gurgel, Rogério Orbetelli, Miko Obradovic, Christian Bader, Christoph Klaus, Evandro Drigo, Mauricio L. Vaz, Fernando Zanini, Jayme Ortiz, Ovandir Bazan

Introdução: Apresentamos um stent válvula expansível de poliuretano (PU), com design especial para pacientes pediátricos, que poderá ser uma opção as próteses biológicas, em que a calcificação ou incompatibilidade prótese/paciente (mismatch) provoca disfunção, encurtando sua durabilidade.

Objetivo: Avaliar os resultados dos testes físicos, hidrodinâmicos e experimentais do stent válvula expansível de poliuretano, com o intuito de, se aprovada, iniciar os ensaios clínicos.

Métodos: As próteses foram submetidas a testes universais, de amostras de PU, pré e pós-crimpagem: força versus deformação - scanner de varredura de superfície e propriedades mecânicas - teste hidrodinâmico. Utilizando fluxo pulsátil, foram estudados área valvar, gradiente de pressão e regurgitação, em condições hemodinâmicas fisiológicas e de estresse. Dez carneiros foram submetidos a implante de prótese por cateter, em posição pulmonar. Grupos: A: 22mm (7 casos); B: 18 mm (3 casos).

Resultados: I- testes in vitro: o PU mostrou ser uma estrutura termoplástica com alta deformação, resistente ao crimpamento e ao alongamento. O teste hidrodinâmico mostrou baixos gradientes, independentemente do diâmetro da prótese. II- testes in vivo: após 5 meses de implante, o estudo eco 3D mostrou: desempenho hemodinâmico satisfatório, discreto gradiente transvalvar e ausência de regurgitação e livre de calcificação dos folhetos de PU.

Discussão: As próteses biológicas em pacientes pediátricos têm apresentado disfunção e calcificação precoce, estimulado a procura de outros materiais.

Conclusão: O monitoramento dos resultados favoráveis atesta que o stent válvula de PU pode ser implantado em crianças $<5$ anos durante a cirurgia convencional e > 7 anos, mediante implante por cateter.

\section{PO $04 \cdot 10 \mathrm{~h} 20-10 \mathrm{~h} 25$}

\section{Análise do uso de enxerto de artéria torácica interna bilateral na cirurgia de revascularização do miocárdio como fator de risco para mediastinite}

Rodrigo Coelho Segalote, Bruno Zappa, Giovanna Ferraiuoli, Bernardo Rangel Tura, Alexandre Rouge, Diego Martins, Leonardo Marega, Mansour Chaer Alameddin, Renata Esteves, Bruno Marques

Introdução: $\mathrm{O}$ uso das artérias torácicas internas bilaterais (ATIB) como enxerto na cirurgia de revascularização do miocárdio (RVM) podem ter melhores resultados em relação a apenas uma artéria torácica interna (ATI). Porém, seu uso pode estar relacionado a uma maior incidência de mediastinite.

Objetivo: Analisar os resultados dos pacientes submetidos a cirurgia de RVM com ATIB em comparação à ATI única como fator de risco para mediastinite.

Métodos: Foram operados 2.134 pacientes de 2008 a 2016, sendo 1.491 (70,0\%) do sexo masculino e 642 (30,0\%) do sexo feminino; 726 (34,0\%) pacientes eram diabéticos. Em 125 (5,8\%) pacientes foram utilizadas as ATIB. Os pacientes foram divididos em dois grupos: grupo ATIB e grupo ATI. A análise estatística foi realizada utilizando os testes t de Student, qui-quadrado e regressão logística.

Resultados: A mortalidade operatória global foi de 4,68\%, sendo 1 (0,8\%) paciente do grupo ATIB. A taxa global de mediastinite foi de 4,3\%, 3,2\% no grupo ATIB e 4,3\% no grupo ATI, com P=0,6866. Quando fazemos a análise da mediastinite em separado da idade $(P=0,439)$, sexo $(P=0,1136)$, diabetes $(P=0,7879)$ e índice de massa corporal (IMC) $(P=0,00003)$, observamos que somente o IMC apresenta diferença com significância estatística para pacientes com IMC mais baixo e consequente menor risco de mediastinite

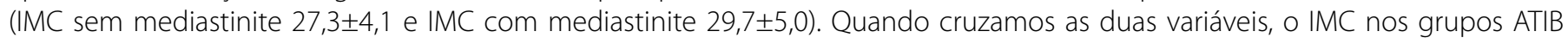
$(26,7 \pm 3,4)$ e ATI $(27,5 \pm 4,2)$, obtemos o $P=0,01955$.

Conclusão: O uso de duas ATI na CRM é seguro, com baixa taxa de mediastinite e resultados comparáveis a uma ATI quando a utilizamos em pacientes com IMC baixo, nesta amostra. 


\section{PO $05 \cdot 10 \mathrm{~h} 25-10 \mathrm{~h} 30$}

\section{Hemodynamic changes during displacement of the heart in the aorta no-touch off-pump coronary artery bypass surgery. A pilot study}

Alexandre Redondo De Carvalho, Walter José Gomes, Solange Guizilini, Gustavo M. Murai, Isis Begot, Isadora S. Rocco, Nelson A. Hossne Jr., Eduardo G. Chamlian, João Manoel T. Santos, Ricardo A. Macedo, Gustavo C. O. Lisboa, Alberto C. Nasciutti, Carlos Eduardo R. Santos, João Paulo M. Figueiredo

Introdução: Aorta no-touch off-pump coronary artery bypass surgery (OPCAB) has been the recommended technique for treatment of patients with high-risk for neurological damage or stroke. However, the displacement of the heart to achieve suitable exposure for graft construction elicits hemodynamic changes, potentially requiring conversion to on-pump.

Objetivo: The aim was to evaluate the sequential changes of hemodynamic parameters in patients underwent aorta no-touch OPCAB.

Métodos: Prospective study involving twenty-seven consecutive patients underwent aorta no-touch OPCAB. The FloTrac/PreSep/ Vigileo system (Edwards Lifesciences) was used to continuously record hemodynamic variables; heart rate (HR), mean arterial blood pressure (MABP), central venous pressure (CVP), continuous cardiac index (FCI), stroke volume (SV), stroke volume variation (VVS), and central venous oxygen saturation ( $\mathrm{ScvO} 2$ ). The parameters were assessed $5 \mathrm{~min}$ before, during and 5 min after each anastomosis (left anterior descending-LAD, posterior descending-PD, marginal-OM and diagonal-Dg). Postoperative lactate was also evaluated.

Resultados: The mean number of anastomoses was 2.7 per patient. No significant change in HR and MABP for all anastomoses, except during PD grafting $(-10.1 \pm 2.7 \mathrm{mmHg}, \mathrm{P}=0.03)$. The SV tended to decrease during the procedure in all territories, but with statistically significant drop only when grafting PD $(-13.6 \pm 5.1, \mathrm{P}=0.007)$. The effects on the $\mathrm{ScvO2}$ were variable, but no change above $10 \%$ was observed. There was significant decrease of ScvO2 only during the $\mathrm{OM}$ anastomoses $(-4.4 \pm 0.4, \mathrm{P}=0.02)$. A drop in CVP after PD manipulation was negatively correlated with postoperative lactate $(r=-0.82, P=0.001)$.

Conclusão: The heart displacement for performing aorta no-touch OPCAB is well tolerated, with transient and endurable hemodynamic variations.

\section{PO $06 \cdot 10 \mathrm{~h} 30-10 \mathrm{~h} 35$}

\section{Drenagem mediastinal posterior prolongada reduz a incidência de reoperação por derrame pericárdico após transplante cardíaco em adultos}

Samuel Padovani Steffen, Fábio Antônio Gaiotto, Domingos Dias Lourenço Filho, Ronaldo Honorato Barros dos Santos, Jorge Edwin Morocho Paredes, Fabiana Goulart Marcondes Braga, Luiz Fernando Bernal da Costa Seguro, Mônica Samuel Ávila, láscara Wosniak, Sandrigo Mangini, Pablo Maria Alberto Pomerantzeff, Fernando Bacal, Ludhmila Abrahão Hajjar, Roberto Kalil Filho, Fabio Biscegli Jatene

Introdução: A incidência de derrame pericárdico é frequente em pacientes que foram submetidos a transplante cardíaco. Estudos demonstram que até $20 \%$ dos pacientes transplantados podem desenvolver derrame pericárdico importante, com necessidade de drenagem por comprometimento hemodinâmico.

Objetivo: Avaliar se o uso adicional de um dreno mediastinal posterior reduz a incidência de derrame pericárdico com comprometimento hemodinâmico e necessidade de reoperação em pacientes adultos submetidos a transplante cardíaco ortotópico.

Métodos: Durante o período de janeiro de 2010 a outubro de 2016, 203 pacientes adultos foram transplantados no Instituto do Coração do Hospital de Clínicas da Faculdade de Medicina da Universidade de São Paulo (InCor-HC-FMUSP), sendo 68 pacientes antes da criação do núcleo de transplantes (2010 a 2013) e 135 pacientes após a criação do núcleo (2013 a 2016). Dentre os 203 pacientes, os primeiros 68 receberam 1 dreno mediastinal e 135 pacientes, 2 drenos mediastinais. O desenvolvimento de derrame pericárdico importante com necessidade de abordagem nos primeiros 30 dias após o transplante foi avaliado e comparado.

Resultados: Após 30 dias do transplante, 28 pacientes desenvolveram derrame pericárdico significativo com indicação de abordagem cirúrgica, sendo 16 pacientes no período anterior à criação do núcleo e 12 no período posterior, correspondendo a 23,5 e 8,8\% dos pacientes transplantados, respectivamente. Houve significância estatística.

Conclusão: $O$ uso de um dreno mediastinal posterior adicional reduziu de forma significativa a incidência de derrame perircárdico com necessidade de reabordagem cirúrgica. Este procedimento simples e seguro tornou-se rotina no núcleo de transplantes do Instituto do Coração. 


\section{PO 07 • 16h05-16h10}

\section{Revascularização miocárdica totalmente arterial da coronária esquerda: utilização da segunda artéria torácica interna para a parede lateral do ventrículo esquerdo}

Nelson Americo Hossne Junior, Eduardo Gregorio Chamlian, Thiago Cavalcanti Vila Nova de Araujo, Ademir Massarico Braz, Caio Cesar Cardoso, Celso Reis de Avila, Fabiano Andre Pereira, Tatiane Lamarca, Walter Jose Gomes

Introdução: Diversos estudos evidenciaram benefício significativo na sobrevida dos pacientes submetidos à cirurgia de revascularizacão miocárdica (RM), quando utilizadas as duas artérias torácicas internas (ATI), em comparação ao uso isolado.

Objetivo: Avaliar uma série consecutiva de pacientes submetidos à RM com a utilização das duas ATI, sendo a segunda ATI para a revascularização da parede lateral do ventrículo esquerdo (PLVE), descrevendo detalhes técnicos intraoperatórios e complicações hospitalares.

Métodos: Pacientes consecutivos submetidos à RM em um centro universitário, de 2013 a 2016, foram incluídos ( $n=142$ ). Os desfechos primários foram pormenorização da técnica operatória da segunda ATI para a PLVE, e incidência de complicações esternais. Desfechos secundários corresponderam às morbidades pós-operatórias.

Resultados: As duas ATI foram empregadas em 122 pacientes (85,92\%), com a segunda ATI para a PLVE em 74 pacientes (52,1\%). Destes, 75,7\% homens (56/74), idade média 60,8 $\pm 8,9$ anos. O número médio de enxertos por paciente foi 2,7 $\pm 0,6$. Na PLVE, a ATI esquerda in situ foi utilizada em 33,8\% dos pacientes, anastomosada para o ramo marginal da circunflexa em 22 pacientes e, para o ramo intermédio, em 3 pacientes. A ATI direita revascularizou a PLVE em 66,2\% dos casos, in situ retroaórtica em 49\% e, como enxerto composto, em Y, da ATIE, em 51\%. Todos pacientes foram operados sem circulação extracorpórea (CEC) e sem manipulacão aórtica. Apenas um paciente evoluiu com infecção superficial de ferida esternal. Não houve infarto do miocárdio, reoperação por sangramento ou óbito.

Conclusão: A utilização da segunda ATI para a revascularização da PLVE pode ser realizada sem CEC ou manipulação aórtica, com bons resultados.

\section{PO 08 • 16h10-16h15}

\section{Análise do fluxo sanguíneo nos enxertos compostos}

Heraldo Guedis Lobo Filho, José Glauco Lobo Filho, Matheus Duarte Pimentel, Bruno Gadelha Bezerra Silva, Camylla Santos de Souza, Marília Leitão Montenegro, Maria Cláudia de Azevedo Leitão, Francisco Vagnaldo Fechine

Introdução: Enxerto composto de artéria torácica interna esquerda (ATIE) e veia safena magna (VSM) na revascularização do sistema coronariano esquerdo (SCE) é uma técnica descrita na literatura desde a década de 1980.

Objetivo: Analisar dinâmica de fluxo sanguíneo neste tipo de enxerto composto, enfocando na adaptabilidade da ATIE e na influência da presença do segmento de VSM sobre o fluxo na ATIE.

Métodos: Vinte e três pacientes receberam enxerto composto em Y de ATIE e VSM, para revascularizar a artéria interventricular anterior (AIA) e outro ramo do SCE, respectivamente, sem circulação extracorpórea (CEC). Fluxo sanguíneo médio, bem como outros critérios de perviedade do enxerto, foram avaliados por fluxometria por tempo de trânsito (FMTT) nos segmentos proximal e distal da ATIE e no segmento de VSM. Mensurações foram realizadas em condição basal e após estresse farmacológico com dobutamina, bem como sem e com clampeamento temporário atraumático (CTA) dos segmentos distais do enxerto composto.

Resultados: Estresse farmacológico resultou em aumento do fluxo sanguíneo em todos os segmentos $(P<0,05)$. CTA do segmento de VSM não causou alteração significativa do fluxo sanguíneo médio no segmento distal da ATIE e vice-versa, tanto em repouso como sob estresse farmacológico.

Conclusão: Análise por FMTT do enxerto composto de ATIE e VSM para revascularização do SCE evidenciou dinâmica de fluxo sanguíneo com adaptabilidade fisiológica em todos os segmentos. A presença do segmento de VSM não alterou a dinâmica de fluxo no segmento distal da ATIE. 


\title{
PO $09_{16 h 15-16 h 20}$
}

\section{Mortalidade associada ao diagnóstico de endocardite infecciosa em um hospital universitário terciário: descrição de uma coorte retrospectiva}

\author{
Gabriela Vieira Steckert, Ana Paula Tagliari, Lucas Molinari Veloso da Silveira, Orlando Carlos Belmonte Wender
}

\begin{abstract}
Introdução: A endocardite infecciosa (El) é uma doença rara, porém com grande relevância, em razão de suas significativas
\end{abstract} morbidade e mortalidade.

Objetivo: Descrever as características epidemiológicas e a mortalidade de pacientes com El em um hospital universitário terciário de 2010 a 2015.

Métodos: Coorte retrospectiva de 81 pacientes diagnosticados com El pelos critérios modificados de Duke. Dados coletados por revisão de prontuários eletrônicos e apresentados como média \pm desvio padrão ou mediana. Mortalidade de acordo com tratamento analisada por teste qui-quadrado.

Resultados: Média de idade de 59,51 anos (15-86 anos), com 67,9\% do sexo masculino. Hipertensão arterial esteve presente em $59 \%$, cardiopatia prévia em 41\%, diabetes em 34\% e cirurgia cardíaca prévia em 29,6\%. Febre foi o sintoma inicial predominante (59\%) e a maioria dos casos era de valva nativa (80,2\%), sobretudo aórtica (58,8\%). Foram encontradas vegetações em 91,4\% dos ecocardiogramas. O germe mais frequentemente encontrado foi o Staphylococcus aureus (19,75\%). Cirurgia foi indicada em 41 casos (50,6\%), predominantemente por ICC descompensada (36,58\%). Óbitos foram significativamente mais prevalentes em pacientes manejados apenas com tratamento clínico quando comparados aos submetidos a tratamento cirúrgico (28-70\% vs. 14$34,14 \% ; P=0,003)$.

Conclusão: Na amostra analisada, pacientes manejados de modo conservador apresentaram maior mortalidade que os tratados cirurgicamente. A elevada taxa de mortalidade associada ao diagnóstico de El mostra a importância de prosseguirmos este trabalho a fim de entender melhor as características epidemiológicas destes pacientes, identificar fatores associados a uma pior evolução e estabelecer novas estratégias terapêuticas visando alterar esses resultados.

\section{PO $10 \cdot 16 \mathrm{~h} 20-16 \mathrm{~h} 25$}

\section{Implementação de um registro clínico prospectivo de cirurgia cardiovascular em adultos}

Bianca Milena Verboski, Sofia Giusti Alves, Kimberlie Gheller, Luis Eduardo Barbosa, Marcus Wachter, Pedro Bretanha, Leonardo Bridi, Renato Abdala Karam Kalil, Clarissa Garcia Rodrigues

Introdução: Registros clínicos são um banco de dados criado a partir da observação focada em uma condição clínica, procedimento, terapia ou população sem abordagens e poucos critérios de inclusão e exclusão.

Objetivo: Descrever a implementação do registro clínico prospectivo de cirurgia cardiovascular em adultos.

Métodos: O questionário do registro clínico foi desenvolvido em um software online de coleta de dados de forma sistematizada, seleção de variáveis com objetivo de integração de dados e definição de seguimento do paciente com avaliação de desfechos.

Resultados: São incluídos os pacientes adultos submetidos a cirurgia cardiovascular no Instituto de Cardiologia do Rio Grande do Sul-Brasil. Contempla: fluxo de trabalho e investigação clínica intra-hospitalar e extra-hospitalar; seleção de variáveis a serem coletadas; desenvolvimento de fichas clínicas eletrônicas; treinamento da equipe clínica para preenchimento e controle de coleta de dados. São características do registro: variáveis padronizadas nacional e internacionalmente (STS Adult Cardiac Database e Registro Brasileiro de Cirurgia Cardiovascular em Adultos); coleta de dados eletrônica (software RedCap); acesso remoto por interface web, gerenciamento central de dados e acessos, diferentes níveis de acesso ao banco de dados, rastreamento de alterações, calendário automático para seguimento, relatórios automáticos de qualidade. Períodos de coleta de dados: avaliação pré, trans e pós-operatório imediato; alta da UTI pós-operatória; alta hospitalar e 1,6 e 12 meses pós-alta.

Conclusão: O registro clínico prospectivo em cirurgia cardiovascular oportuniza a identificação de medidas de avaliação da qualidade assistencial e eficiência em custos. O acompanhamento deste paciente e a interoperabilidade com outros registros em cardiologia internacionais contribuirá para novas descobertas e avanços tecnológicos. 


\section{PO $11 \cdot 16 \mathrm{~h} 25-16 \mathrm{~h} 30$}

\section{Análise do limiar de estimulação e impedância de eletrodos atriais de marca-passos bicamerais comparando eletrodos convencionais com eletrodos que toleram a ressonância magnética: estudo de coorte}

Eduardo Augusto Victor Rocha, João Paulo Cardoso da Silva, Edson Viana Júnior, Paula lara de Souza, Isabela Tiveron Almeida, Wendell Alves Horta, Sérgio Caporali de Oliveira

Introdução: Cerca de 50 a 75\% dos pacientes com marca-passos apresentam alguma indicação para realização de ressonância magnética (RM) ao longo da vida. Não existem estudos sobre o desempenho dos eletrodos que podem ser submetidos à RM.

Objetivo: Avaliar a segurança e o desempenho destes eletrodos para RM comparando com os eletrodos convencionais.

Métodos: Setenta e sete pacientes foram submetidos a implante de marca-passo em instituição única, divididos em dois grupos: eletrodos convencionais e eletrodos de RM. Os limiares de estimulação e impedância foram comparados no momento do implante e com seis meses de pós-operatório.

Resultados: Os grupos foram comparáveis quanto ao gênero, idade e tempo de seguimento. O limiar agudo médio de estimulação foi menor no grupo RM 0,5 mV e de 0,9 mV no grupo convencional ( $P=0,027)$. No limiar crônico não houve diferença significativa, 0,5mV no grupo convencional e $0,6 \mathrm{mV}$ no grupo $\mathrm{RM}(\mathrm{P}=0<765)$. Não houve diferença entre os grupos no que se refere as resistências médias do perioperatório $(P=0,421)$ e crônica $(P=0,925)$. Ambos os grupos reduziram as resistências com seis meses de seguimento $(P<0,0001)$.

Discussão: A mudança da liga metálica não influencia negativamente a análise a longo prazo. Esta influencia parece ser positiva com a redução dos limiares agudos. A resistência elétrica se reduziu ao longo do tempo em ambos os grupos, o que pode ser explicado pela presença de corticosteroide eluído nos eletrodos.

Conclusão: Os eletrodos com novas ligas para RM apresentaram desempenho igual ou superior aos eletrodos convencionais. Houve redução das resistências elétricas tardias em ambos os grupos.

\section{PO $12 \cdot 16 \mathrm{~h} 30-16 \mathrm{~h} 35$}

\section{Avaliação do treinamento de residentes em simulador não biológico de baixíssimo custo}

Roberto Rocha E. Silva, Artur Lourenção Junior, Maxim Goncharov, Fabio Biscegli Jatene

Introdução: Foi desenvolvido um simulador não biológico de baixíssimo custo para treinamento de técnicas simples e complexas em cirurgia cardiovascular.

Objetivo: Avaliação prospectiva das habilidades dos residentes em exercícios básicos neste simulador.

Métodos: O simulador foi confeccionado com caixa "Tupperware" transparente. Foram avaliados exercícios básicos realizados com simuladores de aorta (bandeja de silicone para gelo), safena e coronária (bexiga de festa tipo espaguete). As notas de 1 a 5 foram baseadas na simetria da distância entre pontos e da distância destes pontos em relação à borda da estrutura. Foram obtidas médias dos resultados individuais dos testes de seis residentes e foi medido o coeficiente de correlação linear de Pearson para o efeito de repetições sucessivas do teste. Análises separadas foram feitas para os cinco exercícios estudados que foram realizados de forma sequencial: aortorrafia (6 repetições); botão aórtico (até 12 repetições); sutura em bolsa (até 12 repetições); anastomose coronariana proximal (até 24 repetições); anastomose coronariana distal (até 11 repetições). Foi adotado o valor de P<0,01 como limite de significância.

Resultados: Os seis residentes avaliados apresentaram melhora progressiva e significativa de suas notas $(P<0,01)$ para aortorrafia, botão aórtico, sutura em bolsa e anastomose coronária proximal. Não foi observado progresso no desempenho dos participantes em relação à anastomose coronariana distal $(P>0,2)$.

Discussão: Ao treinarem anastomose proximal previamente, os residentes podem ter iniciado o treinamento de anastomose distal com habilidades adequadas.

Conclusão: Exercícios no simulador baseado em tecidos não biológicos de baixíssimo custo promoveram progresso das habilidades dos residentes. 


\section{PO $13 \cdot 10 h 05-10 h 10$}

\section{Resultados de pacientes submetidos a implante transcutâneo da válvula aórtica (TAVI) no cenário de cuidados intensivos}

Simoni Chiarelli da Silva Pokorski, Luana Claudia Jacoby Silveira, Claudia Motta Mussi, Priscilla Ferreira Saldanha Luana Claudia Jacoby Silveira, Joseane Andrea Kollet Augustin, Dayanna Machado Pires Lemos, Orlando Carlos Belmonte Wender, Eduardo Keller Saadi, Eneida Rejane Rabelo da Silva

Introdução: O implante transcateter de válvula aórtica (TAVI) tem sido considerado o tratamento de maior efetividade para pacientes com alto risco cirúrgico. Como é uma técnica recentemente implementada, a demanda dos cuidados dos pacientes diante das complicações mais frequentes tem sido um desafio para a equipe de enfermagem.

Objetivo: Descrever o tempo de internação na unidade intensiva e as principais complicações no pós-operatório de TAVI.

Métodos: Estudo transversal que incluiu pacientes com estenose aórtica grave, de ambos os sexos, submetidos a TAVI no período de maio de 2013 a outubro de 2016 em hospital público universitário da região Sul.

Resultados: Foram incluídos 18 pacientes com média de idade $83,2 \pm 5,0$ anos, $61 \%$ do sexo feminino, com permanência média de 4,8 1,4 dias em leito de cuidados intensivos. Entre as complicações do pós-operatório prevaleceram congestão (66,6\%), uso de marca-passo temporário (55,5\%), delirium (50\%), hipotensão (38,9\%) bloqueio de ramo esquerdo (27,7\%), necessidade de marcapasso definitivo $(27,7 \%)$ e sangramento do sítio de punção $(27,7 \%)$.

Conclusão: Ao final de três anos prestando assistência de enfermagem aos pacientes submetidos a TAVI, concluiu-se que esse é um cenário de importante atuação multiprofissional, no qual um acurado exame clínico pelo enfermeiro pode ser capaz de identificar precocemente as principais complicações associadas a este procedimento e, dessa forma, implementar intervenções que possam ser efetivas para evitar, minimizar e controlar os eventos indesejáveis. O desenvolvimento de protocolos assistenciais pode ser uma ferramenta para corroborar este cenário.

\section{PO 14・ 10h10-10h15}

\section{Comunicação interventricular residual após reparo cirúrgico - prevalência, significância e evolução}

Acrisio Sales Valente, Gabriella Cristina Coelho Brito, Manuela Sobral Bentes de Melo, Carlos Henrique Maia Ferreira Alencar, Felipe Leite Simão, Klébia Castelo Branco, Roberto Augusto Carneiro de Mesquita Lobo

Introdução: Os defeitos do septo interventricular (CIV), em sua forma isolada ou associados a outras lesões, constituem as cardiopatias congênitas mais comuns e, na maioria das vezes, requerem tratamento cirúrgico. No entanto, a incidência de CIV residual não é desprezível, podendo chegar a 30\% e permanecendo motivo de questionamentos sobre sua relevância e conduta.

Objetivo: Avaliar a prevalência, importância clínica e evolução das CIVs residuais em pacientes submetidos à correção de CIV isolada, tetralogia de Fallot (T4F) e defeito do septo atrioventricular forma total (DSAVT).

Métodos: Estudo retrospectivo, observacional, longitudinal. Foram avaliados os prontuários de pacientes pediátricos (0-18 anos), submetidos a tratamento cirúrgico para correção de CIV, T4F e DSAVT no Hospital do Coração de Messejana no período de janeiro de 2011 a janeiro de 2015. Foram avaliadas a incidência de CIVs residuais, distribuição por sexo, peso e idade, assim como fechamento espontâneo e evolução clínica. Tratamento estatístico foi realizado para comparação entre grupos.

Resultados: Grupo CIV (108) - 48\% CIVs residuais, 79,1\% < 3 mm, 71,15\% < 10 kg. Em 1 ano, fechamento em 25\%. Grupo T4F (36) - 56\% CIVs residuais, 74,28\% < 3 mm, 54,8\% < 10 kg.Em 1 ano, 30,5\% fecharam espontaneamente. Grupo DSAVT (24) - 63\% CIVs residuais, 75\% < 3 mm. Em 1 ano, 29,1\% fecharam. Sem internações por insuficiência cardíaca, endocardite ou fechamento cirúrgico.

Conclusão: A incidência de CIVs residuais foi alta e sem repercussão clínica no estudo para o período avaliado. 


\section{APRESENTAÇÃO DE PÔSTERES - SBCCV}

\section{PO $15 \cdot 10 \mathrm{~h} 15-10 \mathrm{~h} 20$}

\section{Experiência inicial em cirurgia cardíaca vídeoassistida}

Gustavo Kikuta, João Carlos Jazbik, Joaquim Henrique de Souza Aguiar Coutinho, Ana Carolina Veltri Pacheco, Henrique Madureira da Rocha Coutinho, Bernardo Ferreira Americano do Brasil, Pedro Verbicário dos Santos Leite

Introdução: Com o desenvolvimento de novas tecnologias, a busca por cirurgias minimamente invasivas cresceu exponencialmente na última década, com resultados satisfatórios principalmente na qualidade de vida pós-operatória, sem comprometer o resultado cirúrgico.

Objetivo: Relatar nossa experiência inicial em cirurgia cardíaca videoassistida.

Métodos: Foram avaliados de forma retrospectiva 28 pacientes no período entre agosto de 2015 e novembro de 2016, sendo 22 casos de abordagem da valva mitral, 5 de comunicação interatrial e 1 de revascularização do miocárdio sem circulação extracorpórea (CEC). Adotamos como variáveis tempos de CEC, pinçamento de aorta, ventilação mecânica, internação hospitalar, débito do dreno e tempo de retorno às atividades habituais.

Resultados: Os tempos de CEC e pinçamento foram, respectivamente, $97 \pm 50$ e $78 \pm 52 \mathrm{~min}$. O débito do dreno foi de $468 \pm 182$ $\mathrm{mL}$. O tempo médio de ventilação mecânica foi de 7 horas. O tempo de internação foi de $5 \pm 3$ dias. Não identificamos infecção de ferida operatória. Em duas ocasiões foi necessária ampliação da toracotomia. Foi constatado 1 óbito em nossa casuística. O retorno às atividades cotidianas ocorreu em aproximadamente 20 dias após a alta hospitalar.

Conclusão: A esternotomia mediana continua sendo o padrão-ouro para abordagem das doenças estruturais do coração; contudo, surgiram opções menos invasivas e tão seguras quanto o acesso tradicional. Nossa experiência inicial evidenciou resultados comparáveis à literatura e, mesmo necessitando de uma maior curva de aprendizado, mostrou-se animadora, principalmente quanto à satisfação estética e recuperação pós-operatória.

\section{PO 16 • 10h20-10h25}

\section{Como predizer complicações em pacientes de alto risco submetidos a procedimentos cardiovasculares? - 0 projeto HiriSCORE}

Maxim Goncharov, Camila Arthur, Luiz Augusto Ferreira Lisboa, Alexander Bogachev-Prokofyev, Sheng-Show Hu, Eduardo Tamayo Gomez, Vadim Kotowicz, Fabiano Vianna, Fernando Atik, Marcelo Nakazone, Elina Vlasova, Pedro Gabriel M. de Barros E Silva, Rodrigo Segalote, Alexandre Hueb, Marcos Tiveron, Omar Asdrúbal Vilca Mejía, Fabio Biscegli Jatene

Introduction: Risk scores are inaccurate to prediction complication in high-risk patients undergoing cardiovascular procedures.

Objetivo: To evaluate the most accurate score to predict the risk of postoperative complications in patients considered high risk by traditional scores.

Methods: An international, multicenter, prospective study for identify high-risk patients in cardiovascular procedures: HiriSCORE Project. Patients with EuroSCOREII $>5$ and/or STS $>5$ are included. The prospective analysis of the 172 perioperative (from admission until 30 days after surgery) variables of patients undergoing cardiovascular procedures (CABG and/or heart valve surgery, ascending aortic procedures and valve surgery or/and $C A B G$, including mini access, hybrid and transcatheter procedures). We use the calibration (Hosmer Lemeshow) and discrimination (ROC curve) tests for assessment performance of EuroSCOREIl and STS.

Results: 208 patients were analyzed (24-90y) in 6 months. The mortality rate was 44 patients (27,3\%). The morbidity rate was 87 $(41,8 \%)$. EuroSCOREII and STS showed significant association with mortality ( $P=0,001 ; P=0,005$, respectively). So far, we can say that STS is better for predicting mortality (ROC: $0,75 \mathrm{vs}$. ROC: 0,66 ) and worse for morbidity (ROC: 0,59 vs. ROC: 0,62) in relation to EUrOSCOREII.

Conclusion: Although EuroSCOREIl and STS score underestimate observed mortality, until now both scores seem to move to be considered acceptable. Underestimate observed mortality is probably due to low number of patients, voluntary register and fear to be exposed. Exists a probability that other studies did not include some high-risk patients, who died during the treatment. We need to continue the study, aimed to risk assessment for the high-risk patients. 


\section{PO 17 - 10h25-10h30}

\section{Concentração de cefazolina no tecido adiposo do mediastino de pacientes submetidos a cirurgia cardíaca}

Rodrigo Mezzalira Tchaick, Fernando Augusto M. Santos Figueira, Kilma Coelho Paz, Michel Pompeu Barros Oliveira Sá, Alvaro Antônio Bandeira Ferraz, Fernando Ribeiro de Moraes Neto

Introdução: A antibioticoprofilaxia é uma das principais medidas de prevenção à infecção. A utilização de vários antibióticos indica que não existe um consenso sobre a eficácia e a segurança de cada um deles.

Objetivo: Avaliar a concentração de cefazolina no tecido adiposo do mediastino e correlacionar o tempo decorrido entre a infusão da cefazolina e a coleta da amostra no inicio e no fim da cirurgia.

Métodos: Coletadas amostras de 1 grama de tecido adiposo de pacientes operados no período de 2014 a 2015. A dosagem do antibiótico nas amostras foi realizada por cromatografia a líquido de alta eficiência por fase reversa.

Resultados: $n=19$. O tempo decorrido entre a infusão do antibiótico até a primeira coleta foi de 26,5 minutos, e o tempo da última coleta com relação à última dose feita foi de 180 minutos. A concentração de cefazolina aferida na primeira amostra foi de 6,01 $\mu \mathrm{g} /$ mL; já na amostra coletada ao final da cirurgia, a concentração média foi de $8,4 \mu \mathrm{g} / \mathrm{mL}$.

Conclusão: Durante todas as cirurgias analisadas, a concentração de cefazolina manteve-se dentro dos padrões de segurança estabelecidos por entidades internacionais, além de existir uma correlação entre o tempo decorrido entre a infusão e a coleta da amostra com a concentração de cefazolina no tecido.

\section{PO $18 \cdot 10$ h $30-10 h 35$}

\section{Anuloplastia tricúspide com anel em pacientes submetidos a cirurgia cardíaca em geral}

Leonardo Secchin Canale, Andrey José Monteiro, Bruno Miranda Marques, Natalia Amarante, Rafaella Cunha

Introdução: A plastia tricúspide para tratamento de insuficiência tricúspide funcional é um procedimento comumente associado em cirurgia cardíaca geral. O uso de anel tem se consolidado como preferência na anuloplastia.

Objetivo: Avaliar segurança e eficácia do anel rígido Star Labcor em plastia tricúspide em cirurgia cardíaca geral no tratamento de insuficiência tricúspide funcional.

Métodos: Quarenta pacientes operados entre 2014 e 2015 por diversas doenças básicas foram submetidos concomitantemente à plastia tricúspide com anel rígido Star Labcor. Ecocardiograma transtorácico foi realizado antes e após o procedimento com o objetivo de avaliar a insuficiência tricúspide. A idade dos pacientes variou de 23 a 69 anos, sendo 55\% mulheres. Procedimentos mitrais foram realizados em $87,5 \%$ dos pacientes. Setenta e cinco por cento dos pacientes tinham etiologia reumática $75 \%$.

Resultados: A mortalidade cirúrgica foi de 7.5\%. A necessidade de marca-passo no pós-operatório foi de 5\%. A insuficiência

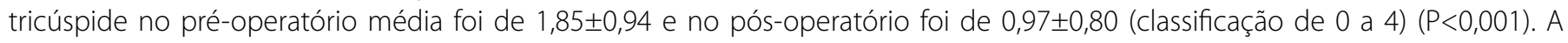
pressão sistólica de artéria pulmonar era de 58,5 mmHg no pré-operatório e de 43,6 mmHg no pós-operatório.

Discussão: A taxa de complicações associadas à plastia tricúspide foi baixa e a melhora da insuficiência tricúspide foi significativa.

Conclusão: O uso do anel rígido Star Labcor é seguro e eficaz no tratamento da insuficiência tricúspide funcional. 


\section{EXPOSIÇÃO DE PÔSTERES - SBCCV}

\section{PO 19}

\section{Experiência Inicial com implante transcateter valve-in-valve em biopróteses degeneradas no Instituto do Coração (InCor) do HC-FMUSP}

Renato Cesar de Souza, Leonardo Paim Nicolau da Costa, José Honório Palma da Fonseca, Joaquim Aprigio Nóbrega Batista, Guilherme Viotto Rodrigues da Silva, Lucas Lacerda Araújo, Carlos Manuel de Almeida Brandão, Pablo Maria Alberto Pomerantzef, Fabio Biscegli Jatene

Introdução: O número de próteses valvares biológicas implantadas tem aumentado nos últimos anos. Consequentemente, espera-se que mais pacientes sejam reoperados por disfunção dessas próteses. O "valve-in-valve" (ViV) transcateter é uma promessa para um futuro próximo na reabordagem dessas próteses disfuncionantes em pacientes de alto risco.

Objetivo: Relatar a experiência inicial com pacientes submetidos a valve-in-valve no InCor - HC-FMUSP no período de 2015 e 2016.

Métodos: Estudo retrospectivo, realizado por meio de revisão de prontuário de todos os pacientes submetidos a ViV no InCor HC-FMUSP nos anos de 2015 e 2016. Totalizaram 19 pacientes de alto risco (15 mitrais, 3 tricúspides e 1 aórtico), com disfunção de prótese biológica, discutidos em sessão clínica com heart team, em que se optou pelo procedimento transcateter.

Resultados: O procedimento proposto foi realizado com sucesso em todos os casos, sem necessidade de conversão para cirurgia convencional. Sangramento importante foi relatado em 2 pacientes, e 1 necessitou de reabordagem cirúrgica. Regurgitação paravalvar moderada ocorreu em apenas 1 paciente. Houve redução do gradiente médio mitral de $11 \mathrm{mmHg}$ para $7 \mathrm{mmHg}$, tricúspide de 10,6 mmHg para 3,7 $\mathrm{mmHg}$ e aórtico de $39 \mathrm{mmHg}$ para $24 \mathrm{mmHg}$. Insuficiência renal aguda ocorreu em 50\% dos pacientes. A mortalidade durante a internação foi de 15,7\%.

Conclusão: O valve-in-valve transcateter é viável e apresenta resultados aceitáveis para pacientes com disfunção de prótese biológica e de alto risco.

\section{PO 20}

Série de casos comparativos entre cirurgia cardíaca minimamente invasiva e a técnica tradicional para abordagem da valva mitral

Gustavo Kikuta, Joaquim Henrique de Souza Aguiar Coutinho, João Carlos Jazbik, Ana Carolina Veltri Pacheco, Henrique Madureira da Rocha Coutinho, Bernardo Ferreira Americano do Brasil, Pedro Verbicário dos Santos Leite

Introdução: Há anos, a abordagem para o tratamento de doenças cardíacas tem como padrão-ouro a esternotomia mediana. Com o advento de novas tecnologias e o aprimoramento dos instrumentais, acessos menos invasivos surgiram como opções em casos selecionados.

Objetivo: Corroborar a factibilidade desta nova proposta cirúrgica comparando-a com o método tradicional.

Métodos: Foram escolhidos como critérios de exclusão para a realização da cirurgia videoassistida o grau de calcificação do anel mitral, pressão sistólica em artéria pulmonar (PSAP), presença de doença cirúrgica concomitante, doença arterial periférica e cirurgia prévia em hemitórax direito. Para avaliação, foram adotados tempo de circulação extracorpórea (CEC) e pinçamento de aorta, débito do dreno, tempo de ventilação e internação.

Resultados: No G1 os tempos de CEC e pinçamento foram de $96 \pm 24$ e $77 \pm 23$ min, respectivamente, débito do dreno de $480 \pm 875$ $\mathrm{mL}$, tempo de ventilação de $7 \pm 7$ horas e período de internação $6 \pm 2$ dias. No G2 os tempos de CEC e pinçamento foram de $73 \pm 46 \mathrm{e}$ $58 \pm 34$, débito do dreno de $497 \pm 878 \mathrm{~mL}$, tempo de ventilação de $7 \pm 12$ horas e período de internação de $8 \pm 10$ dias. No G1 o retorno às atividades ocorreu após 21 dias da cirurgia, ao passo que no G2 ocorreu após 3 meses.

Conclusão: Apesar dos tempos de CEC e pinçamento aórtico maiores, constatamos que a cirurgia videoassitida é segura, principalmente ao observar a rápida recuperação pós-operatória. Em relação à satisfação estética, observaram-se índices superiores aos do método tradicional. 


\section{PO 21}

\section{Transplante cardíaco heterotópico: a experiência em Alagoas}

Marina Mendes Melo, Bruna Gomes de Castro, João Vitor Campos da Silva, Mariana Ramos Andion, Vanessa Késsia Silva Texeira de Moura, Rafaela da Hora Sales, José Leitão da Silva Neto, Peter Conde Vidal Junior, Francisco Siosney de Almeida Pinto, José Wanderley Neto

Introdução: O transplante heterotópico tem sido encarado como um procedimento de exceção, reservado a pacientes com hiperresistência pulmonar ou desproporção ponderal doador/receptor. Considera-se a possibilidade do uso de corações marginais e posterior realização de cirurgias adicionais no coração nativo, melhorando sua performance.

Objetivo: Demonstrar a experiência do serviço nesse procedimento por meio do relato dois casos em que houve intervenção no coração nativo durante o procedimento de transplante, sendo os primeiros relatos no Brasil.

Métodos: Estudo descritivo, observacional e transversal com base nos prontuários no período de setembro de 1999 a junho de 2011.

Resultados: Foram operados 5 pacientes do sexo masculino com idades entre 29 a 60 anos, portadores de ICC refratária e NYHA IV, 3 deles com miocardiopatia idiopática e 2 com miocardiopatia isquêmica, com peso de 70 a $125 \mathrm{~kg}$. Dois pacientes tinham cirurgias prévias, como aneurismectomia de ventrículo esquerdo (VE), CRM e troca de valva mitral. Os doadores eram mulheres com baixo peso, sendo 4 consideradas marginais. Foram realizadas cirurgias adicionais no coração de dois pacientes, aneurismectomia e redução de VE. No pós-operatorio, ocorreram 3 episódios de infecção, 1 AVE sem sequela e 1 trombo no VE do coração nativo sem consequências. Não houve óbitos no pós-operatorio imediato. Houve 4 óbitos durante seguimento, causados por neoplasias, obesidade mórbida e morte súbita em dois pacientes por falta de adesão ao acompanhamento.

Conclusão: O transplante cardíaco heterotópico é uma alternativa segura nas indicações clássicas, sendo possível ampliar a técnica em corações nativos e aumentando o número de transplantes realizados.

\section{PO 22}

\section{Uso da artéria torácica interna direita como opção na revascularização do miocárdio com múltiplos enxertos arteriais}

Samuel Soares Eduardo, Andreza Sérvula Pereira da Silva, Paulo Marcelo Barbosa Mesquita, Pedro Antonio Gomes Maciel

Introdução: A utilização de enxertos arteriais iniciou-se com a artéria torácica interna esquerda, que hoje é padrão-ouro nesse procedimento. A cirurgia de revascularização do miocárdio (RM) com duas artérias torácicas internas (ATI) como enxerto tem sido associada a melhor sobrevida em longo prazo e diminuição de eventos cardiovasculares.

Objetivo: Avaliar a aplicabilidade do uso das duas ATI na cirurgia de RM.

Métodos: Estudo analítico, retrospectivo. Coleta de dados dos prontuários de pacientes submetidos à cirurgia de RM em que houve uso da artéria torácica interna direita (ATID), no período de janeiro de 2013 a outubro de 2016 no serviço de referência em cirurgia cardíaca no interior do Ceará.

Resultados: Foram realizadas 271 cirurgias de RM isolada em que mais de uma coronária foi revascularizada. A idade média foi 63,3 anos. Em 114 (42,0\%), a ATID foi utilizada como opção além da ATIE. Em 62,6\% a ATID foi usada para o sistema esquerdo, em 31,7\% para o sistema direito, e em 5,7\% para ambos. Para o sistema esquerdo, a configuração mais utilizada foi a composição em "Y" em 77,9\% (60), in situ 14,2\% (11). Para o sistema direito, a composição em "I" foi mais comum, em 66,6\% (26) - com artéria radial 46,1\% ou com veia safena 53,9\%, in situ em 33,3\% (13). Todas ATI foram dissecadas de forma esqueletizada. Não houve complicações esternais nesses pacientes.

Conclusão: O uso de ambas as ATI é uma boa opção como estratégia de revascularização do miocárdio com enxertos arteriais múltiplos. A utilização da ATID não aumentou o número de complicações esternais. 


\section{EXPOSIÇÃO DE PÔSTERES - SBCCV}

\section{PO 23}

\section{Tratamento exclusivamente endovascular de aneurisma dissecante de aorta tipo A com envolvimento da crossa e aorta descendente via transapical e transfemoral com endoprótese nacional trifurcada (Braile Biomédica)}

Luiz Fernando Kubrusly, Fernando B. Kubrusly, Andressa S. Bertoldi, Caroline F. Santos, Emily J. Carvalho, Camila Marques, Carolina B. N. Duarte Ferreira, Diego Gaia, Jose Honório Palma

Introdução: Dissecções aórticas tipo A envolvendo crossa e aorta descendente têm técnicas cirúrgicas a céu aberto e híbridas com morbidade e mortalidade altas. O tratamento exclusivo endovascular apresenta-se como desafio pela revascularização dos vasos da base. Não existem relatos de tratamento exclusivo endovascular da dissecção tipo A com preservação dos vasos supraórticos.

Objetivo: Relatar o primeiro caso de tratamento exclusivamente endovascular de aneurisma dissecante tipo A envolvendo crossa e aorta descendente, via transapical e transfemoral com endoprótese nacional trifurcada para vasos supraórticos.

Métodos: Paciente, 65 anos, hipertensa grave, em 2015 apresentou úlcera e dissecção aórtica (tipo B) com falsa luz até mesentérica superior. Indicado tratamento endovascular, recusado pela paciente, sendo acompanhada clinicamente. Com seis meses de evolução desenvolveu dissecção retrógrada para crossa, subclávia e carótida esquerdas, tronco braquicefálico e aorta ascendente supracoronária. Foi confeccionada endoprótese nacional trifurcada, testada em modelo 3D de prototipagem rápida, implantada via transapical na aorta ascendente, crossa e início da descendente. A prótese constituía-se de um corpo principal e ramos de Viabanh . $\mathrm{Na}$ via transfemoral foi implantada endoprótese reta acoplada àquela trifurcada. Os ramos supraórticos foram revascularizados seletivamente com punções carotídeas bilaterais.

Resultados: Em 12 meses a paciente encontra-se assintomática, sem endoleaks ou sinais de isquemia ou oclusão dos ramos.

Conclusão: O tratamento exclusivamente endovascular da aorta ascendente, crossa e descendente, antes um paradigma, foi possível com a revascularização dos três vasos supra-aórticos, com prótese nacional customizada, trifurcada, com excelente resultado.

\section{PO 24}

\section{Reoperação de valva mitral por procedimento minimamente invasivo}

Jeronimo Antonio Fortunato Junior, Jerferson R. Sesca, Wilson Nkundumukisa

Introdução: Lesões das estruturas cardíacas durante liberação de aderências em reoperações estão associadas a aumento da morbidade e mortalidade.

Objetivo: Analisar os resultados pós-operatórios com o uso da cirurgia cardíaca minimamente invasiva (CCMI) em reoperação de valva mitral sem dissecções ou clampeio da aorta comparando com um grupo similar de CCMI de primeira intervenção.

Métodos: Vinte cirurgias de troca da valva mitral foram realizadas por mínimas incisões. Neste grupo,10 eram reoperações (G1) e foram comparados aos de primeira troca (G2). A distribuição demográfica foi semelhante em ambos os grupos, à exceção da idade mais elevada no grupo das reoperações $(40,1 \pm 3,6$ contra 49,8 $\pm 14,7$ anos [P=0,04]). Todos foram operados por minitoracotomia anterolateral direita e submetidos à canulação periférica através dos vasos femorais. Foi adotada hipotermia sistêmica com fibrilação ventricular.

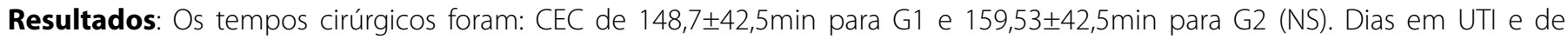

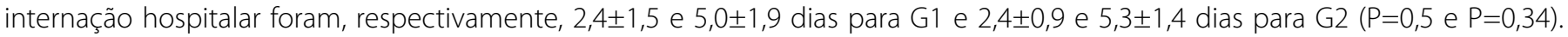

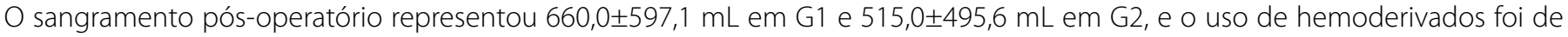

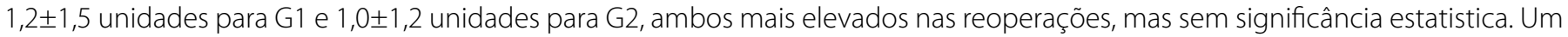
óbito inicial ocorreu em G1 e 1 óbito tardio ocorreu em cada grupo.Não houve reoperação tardia e a sobrevida após um período médio de cinco anos foi de 91,66\%.

Conclusão: Nesta análise, pudemos demonstrar que o uso de CCMI para reoperações da valva mitral não diferiu dos resultados encontrados para a primeira cirurgia com a mesma técnica, podendo ser usada com segurança e eficácia nestas condições. 


\title{
PO 25
}

\section{Fibrilação atrial nova em pós-operatório precoce de cirurgia cardiovascular: coorte prospectiva em um hospital universitário}

\author{
Ana Paula Tagliari, Adriano Nunes Kochi, Anderson Castro de Souza, Orlando Carlos Belmonte Wender
}

Introdução: Fibrilação atrial (FA) é a arritmia cardíaca mais prevalente e complicação de maior incidência no pós-operatório de cirurgia cardiovascular (PO-CCV), afetando até $60 \%$ dos pacientes submetidos à cirurgia valvar com CRM e se associando a maiores taxas de acidente vascular cerebral (AVC), tempo de hospitalização e mortalidade total.

Objetivo: Avaliar a incidência de FA no PO-CCV precoce (até a alta hospitalar) e a incidência do desfecho morte, AVC ou tempo prolongado (> 72 horas) de internação em unidade de terapia intensiva (UTI).

Métodos: Coorte prospectiva, unicêntrica, de maio de 2015 a junho de 2016. Variáveis contínuas expressas como média desvio padrão e comparadas com teste t de Student e categóricas como percentual e avaliadas com teste qui-quadrado ou exato de Fisher.

Resultados: Foram avaliados 158 pacientes, sendo 20 excluídos por FA prévia. Observaram-se 31 casos de FA nova (22,5\%), os quais apresentaram maior idade média $(60,1 \pm 12,9$ vs. $67,6 \pm 9,4 ; P=0,003)$. Sexo masculino (18,3\% vs. 30,8\%), CCV prévia (0 vs. 25,4\%), AVC

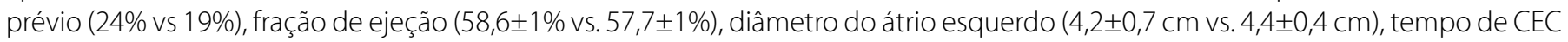
( $82 \pm 3 \mathrm{~min}$ vs. $88 \pm 3 \mathrm{~min})$ e isquemia $(64,1 \pm 38,8 \mathrm{~min}$ vs. $68,5 \pm 28,7 \mathrm{~min})$, tipo de CCV (CRM, valvar ou CRM+valvar) $(20,3 \%$ vs. $28,2 \%$ vs. 35,3\%) não diferiram. Quanto ao desfecho primário, FA nova aumentou significativamente a chance de óbito precoce, AVC ou internação em UTI (44,3\% vs. 74,2\%; $P=0,004)$.

Conclusão: Em coorte prospectiva de pacientes em PO-CCV, a incidência de FA esteve associada a maiores taxas de morte, AVC ou tempo prolongado de internação em UTI.

\section{PO 26}

\section{Experiência na extração de eletrodos endocárdicos com o uso de dilatador mecânico em um hospital público de referência}

Julianny Freitas Rafael, Rodrigo Minati Barbosa, Gustavo de Castro Lacerda

Introdução: O número de pacientes submetidos a implante de dispositivos de estimulação cardíaca artificial tem crescido de modo significativo, assim como as indicações de extração de eletrodos endocárdicos. A técnica de contratração transvenosa com o uso de dilatador mecânico facilita a extração desses eletrodos sem a necessidade de toracotomia.

Objetivo: Relatar a experiência de um centro de referência do Ministério da Saúde com a extração de eletrodos endocárdicos.

Métodos: Foram avaliados retrospectivamente todos os procedimentos de extração percutânea de eletrodos endocárdicos de janeiro de 2014 a julho de 2016. O sucesso do procedimento, assim como complicações maiores e menores, foi determinado de acordo com o preconizado na diretriz brasileira.

Resultados: Dezesseis pacientes foram submetidos à extração percutânea de 25 eletrodos endocárdicos, sendo 12 atriais, 7 ventriculares convencionais, 3 ventriculares com duplo coil de choque, e 3 do seio coronariano. O eletrodo mais antigo havia sido implantado há 22 anos, e o mais novo, há 1 ano. Em 81,2\% a extração foi por infecção e em 18,75\%, por disfunção do eletrodo. Sucesso radiológico completo foi obtido em $88 \%$ dos casos, parcial em $4 \%$, e houve falha na extração em $8 \%$. Sucesso clínico ocorreu em $87,5 \%$ e falha clínica, em 12,5\%. Complicações maiores ocorreram em 6,25\% e também em 6,25\% houve ocorrência de óbito. Complicações menores ocorreram em 18,75\%.

Conclusão: A extração de eletrodos endocárdicos, com longa duração do implante, principalmente como recurso terapêutico em pacientes infectados, em um centro de referência, vem sendo realizada de forma segura e com sucesso, utilizando dilatadores mecânicos. 


\section{EXPOSIÇÃO DE PÔSTERES - SBCCV}

\section{PO 27}

\section{Troca valvar aórtica e aneurismectomia em paciente com anemia falciforme: um relato de caso}

Lucas Molinari Veloso da Silveira, Ana Paula Tagliari, Ronaldo David da Costa, Cristiano Blaya Martins, Orlando Carlos Belmonte Wender

Introdução: Pacientes com anemia falciforme submetidos a cirurgias cardiovasculares com circulação extracorpórea (CEC) estão sujeitos a diversas complicações decorrentes deste procedimento.

Objetivo: Relatar caso de troca valvar aórtica com aneurismectomia em paciente com anemia falciforme.

Métodos: Paciente masculino, 30 anos, com anemia falciforme SS e diagnóstico ecocardiográfico prévio de insuficiência aórtica grave, apresentou-se com quadro de crise falcêmica e insuficiência cardíaca descompensada. Angiotomografia de aorta torácica demonstrou dilatação em aorta ascendente, medindo $48 \mathrm{~mm}$. No manejo pré-operatório, foi planejada exsanguinitransfusão no dia anterior à cirurgia visando hemoglobina S menor que 30\%. Transfusões sanguíneas foram realizadas diariamente, alcançando o valor preestabelecido de hemoglobina S, não necessitando, assim, da realização de exsanguinitransfusão.

Resultados: O paciente foi submetido a cirurgia de troca valvar aórtica e aneurismectomia de aorta ascendente. Durante a CEC, foi mantido em normotermia $\left(34^{\circ} \mathrm{C}\right)$. Ademais, foram evitadas hipóxia e desidratação durante o procedimento. Foram implantadas prótese biológica bovina $n^{\circ} 25$ e tubo de Dacron $n^{\circ} 26$ supracoronariano. No pós-operatório imediato, desenvolveu choque hipovolêmico, sendo realizada mediastinotomia exploradora. O ecocardiograma demonstrou fração de ejeção de $29 \%$ e prótese normofuncionante com gradientes de 15/9 mmHg. Apresentou boa recuperação, tendo alta no $18^{\circ}$ dia de pós-operatório. Em seguimento ambulatorial, demonstrou melhora sintomática significativa.

Conclusão: Acreditamos ser importante o relato deste caso, pois os pacientes com anemia falciforme estão sujeitos a maiores complicações após cirurgias cardiovasculares. Desta forma, necessitam de preparo pré-operatório diferenciado, sendo este caso um exemplo de tratamento bem-sucedido de condição complexa e pouco relatada na literatura.

\section{PO 28}

\section{Resultados imediatos do emprego de miniesternotomia para troca valvar aórtica}

Ricardo Riet Vargas Langenegger, Paulo Roberto Lunardi Prates, Lucas Krieger Martins, Sofia Giusti Alves, Paula Linck de Mello Nesralla, Alexsandra Balbinot, Alvaro Schmidt Albrecht, Rogerio de Souza Abrahão, Flavio Peixoto de Oliveira, Guaracy Teixeira Filho, João Ricardo Michielin Santanna, Paulo Roberto Prates, Ivo Abrahão Nesralla, Renato Abdala Karan Kalil

Introdução: Técnicas minimamente invasivas para troca valvar aórtica, como a miniesternotomia superior, são seguras. Entretanto, ainda existe espaço para descrever resultados atuais do nosso meio.

Objetivo: Descrever a experiência de um centro de referência no uso de miniesternotomia em J para troca valvar aórtica, por meio da análise de série de casos retrospectiva recente.

Métodos: Foram revisados prontuários de 2013 a 2016 de pacientes submetidos à troca valvar aórtica por miniesternotomia. Foram analisados dados nos períodos de internação pré e pós-operatória. Foram encontrados 42 pacientes (37 a 86 anos), predominantemente com estenose valvar $(90,48 \%)$. Comorbidades mais frequentes: fibrilação atrial=11,9\%; DBPOC= 11,9\%; tabagismo=35,71\%. Tempo de internação pré-operatório: 1 a 49 dias. Sintomáticos: 47\%. Possuíam disfunção ventricular esquerda: $61,9 \%$.

Resultados: Não houve dificuldades de abordagem valvar, nem sangramento transoperatório aumentado. Tempo médio de circulação extracorpórea 86 minutos e pinçamento aórtico 68 minutos. Principal complicação no pós-operatório: fibrilação atrial aguda (26,19\%). Houve uma reoperação por sangramento, um acidente vascular cerebral e um óbito (2,38\%). Mediana de tempo de internação total: 10 dias. Mediana de tempo da cirurgia até a alta hospitalar: 8 dias. Alguns pacientes tiveram o tempo de internação total fortemente influenciado por questões não cirúrgicas. Reconhecemos as limitações do estudo retrospectivo, porém consideramos estes dados úteis e estamos dando seguimento na comparação com a cirurgia convencional e implantes percutâneos na instituição.

Conclusão: A miniesternotomia para troca valvar aórtica mostrou-se uma técnica segura, mesmo em pacientes debilitados. Há necessidade de prosseguir com o estudo para comparação com as técnicas tradicional e percutânea de troca valvar aórtica. 


\section{PO 29}

\section{Oclusão percutânea da comunicação interatrial com uso de ecocardiograma intracardíaco}

Vitória Mikaelly da Silva Gomes, Debora Irene Barbosa, Gabriel Silvestre Minucci

Introdução: O cateterismo terapêutico da comunicação interatrial (CIA) tem obtido sucesso desde que foi descrito pela primeira vez por King e Mills em 1974. O uso do ecocardiograma transesofágico (ECO-TE) é a forma mais usada para guiar o fechamento percutâneo, mas o ecocardiograma intracardíaco (ECO-IC) tem surgido como uma nova alternativa na obtenção de boas imagens e garantia do sucesso terapêutico.

Objetivo: O objetivo deste estudo foi analisar a oclusão percutânea com uso do ECO-IC e seu impacto na CIA.

Métodos: Foi realizada uma revisão da literatura nas bases de dados da Biblioteca Virtual de Saúde, em novembro de 2016. Foram usados os descritores Percutaneous occlusion in the CIA e Percutaneous occlusion with echocardiogram in the CIA, sendo encontrados 16 artigos na plataforma MEDLINE.

Resultados: A técnica de oclusão percutânea é considerada tratamento de escolha na CIA. Nesse cenário, a ecocardiografia é usada na identificação dos candidatos para o procedimento e na monitorização do implante da prótese. O ECO-IC mostra-se superior ao ECO-TE por permitir a obtenção de imagens de melhor qualidade, melhor visualização da região posteroinferior do septo interatrial, exposição diminuída à radiação, além de não necessitar de anestesia geral e não exigir um operador específico para o procedimento. Apesar do risco de formação de fístulas arteriovenosas e sangramentos perivasculares, é realizado de forma simples e possui curva de aprendizagem curta.

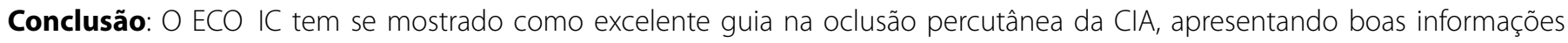
anatômicas e eliminando as desvantagens do ECO-TE.

\section{PO 30}

\section{Dissecção da aorta associada à doença arterial coronariana}

Guilherme Andrade Krawczun, Alexandre Noburu Murakami, Rogerio Toshio Teruya, Leonardo Peixoto Villar, Celso Otaviano Cordeiro, Francisco Gregori Jr.

Introdução: Permanece controversa a indicação de coronariografia em pacientes que serão submetidos à cirurgia cardíaca de dissecção da aorta. Foi feito um levantamento de 158 pacientes operados de dissecção da aorta nos últimos 10 anos, e a incidência de doença coronariana (DAC) não foi infrequente, incluindo casos críticos e triarteriais.

Objetivo: $O$ estudo sugere realizar cateterismo em casos de dissecção da aorta em decorrência da frequente doença coronariana associada.

Métodos: Os 158 casos incluídos neste levantamento foram os pacientes operados de 2006 até a atualidade de correção de dissecção da aorta.

Resultados: O desfecho principal deste estudo concluiu que, dos 158 pacientes operados, 26 (16,4\%) eram portadores de doença coronariana aterosclerótica que necessitou de revascularização de um ou mais vasos. A idade média dos pacientes que necessitaram de revascularização por DAC foi de 61 anos.Quanto ao número de vasos revascularizados, 10 pacientes necessitaram revascularizar 1 vaso (38,4\%), 6 pacientes necessitaram revascularizar dois vasos (23,2\%) e 10 pacientes necessitaram revascularizar 3 vasos (38,4\%). Dentre esses casos, os mais críticos foram um paciente portador de lesão de tronco e outro com lesão de $99 \%$ em descendente anterior.

Conclusão: Conclui-se que a revascularização do miocárdio não é infrequente em pacientes submetidos à correção de dissecção da aorta, portanto, consideramos justificável o estudo hemodinâmico prévio à cirurgia desses pacientes. 


\section{EXPOSIÇÃO DE PÔSTERES \\ - SBCCV}

\section{PO 31}

\section{Estudo dos resultados de implante valve-in-valve tricúspide transcateter}

Camylla Santos de Souza, Raphaella von Sohsten Calabria Lima, Caio Teixeira dos Santos, Ivan Lucas Picove Borges dos Anjos, Ana Carolina Ramos Queiroz, José Hiago de Freitas Damião, Lívia Motta Leitão, Rebeca Carlstom Santos, Daiana Vieira Gomes, Vitória Mikaelly da Silva Gomes, Igor Rodrigues da Silva, Lara Ferreira Ventura, Francisco Siosney Almeida Pinto, José Wanderley Neto, Bruno Miranda Marques, João David de Souza Neto, Diego Felipe Gaia dos Santos

Introdução: Lesões valvares tricúspides podem demandar substituição protética, preferencialmente com próteses biológicas, as quais, apesar dos bons resultados, apresentarão disfunção ao longo da evolução, necessitando de reoperação e elevando substancialmente os riscos. Neste contexto, a possibilidade de implante valve-in-valve (VIV) tem surgido como alternativa mais segura, reduzindo o uso de hemoderivados, tempo de internação e morbidade e mortalidade.

Objetivo: Analisar a experiência com VIV tricúspide transcateter.

Métodos: Revisão de literatura e de dados dos pacientes submetidos à técnica.

Resultados: Realiza-se VIV tricúspide em pacientes idosos ou mais jovens com alto risco cirúrgico, encontrando-se casos entre 8 a 74 anos. De 2010 a 2012, publicaram-se os primeiros 14 casos no mundo. No Brasil, de 2011 até agora, realizaram-se 5 VIVs tricúspides: 4 na Escola Paulista de Medicina (1 transatrial e 3 transjugulares) e 1 transjugular no Instituto Nacional de Cardiologia. A abordagem transatrial demonstra-se "confortável", com fácil passagem da prótese e alinhamento perfeito. A transjugular é preferida pela proximidade com a valva lesionada e por requerer apenas pequena incisão cervical, sem abordagem torácica, diminuindo complicações. Outra técnica é a transfemoral, mais desafiadora pela maior extensão percorrida pelo cateter e pela curva acentuada entre cava inferior e ventrículo direito. Na estenose tricúspide, reduz-se o gradiente médio de 10,8 para 3,6 mmHg, ao passo que na insuficiência há melhora completa. Sobre as próteses, existem a Melody, a Edwards SAPIEN e a Braile Inovare. Esta, disponível em tamanhos variados, favorece melhor escolha, evitando mismatch. Todas apresentam os mesmos riscos de embolização.

Conclusão: O VIV tricúspide é extremamente promissor, melhorando o prognóstico dos pacientes de alto risco.

\section{PO 32}

\section{Comparação entre a taxa de mortalidade e o perfil de óbitos por aneurisma e dissecção de aorta entre as regiões do Brasil}

Clara Aguiar Mendes, Ana Eloísa Melo Novaes, Henrique de Paula Bedaque, Júlio César Vieira de Sousa

Introdução: As síndromes aórticas agudas representam um acometimento potencialmente fatal que exige muito do sistema de saúde. Conhecer o perfil dos óbitos por essa síndrome pode nortear condutas mais efetivas.

Objetivo: $O$ estudo teve como objetivo comparar as taxas de mortalidade por síndromes aórticas e o perfil epidemiológico dos óbitos nas diferentes regiões do Brasil, por meio de um estudo transversal descritivo analítico.

Métodos: Compararam-se a taxa de mortalidade e o perfil de óbitos (sexo, faixa etária e cor/raça) decorrentes de síndromes aórticas entre as regiões do Brasil. Foram coletados dados no portal DATASUS e do IBGE.

Resultados: A taxa de mortalidade decorrente de síndromes aórticas cresceu em todo país, exceto no Sul (redução de 5,78\%). A região Norte apresentou o maior aumento (119\%) para o período estudado, seguida pelo Nordeste (114\%), Centro-Oeste (42\%) e Sudeste (34\%). Com relação ao perfil epidemiológico, a maioria dos óbitos ocorre no sexo masculino, predominando cor/raça branca no Centro-Oeste, Sul e Sudeste, e parda no Norte e Nordeste. Ademais, prevaleceu a faixa etária de 70 a 79 anos na maioria das regiões, com exceção do Centro-Oeste.

Conclusão: Os dados coletados demonstram que, apesar do desenvolvimento de sofisticados métodos de diagnóstico e tratamento, óbitos por síndromes aórticas continuam a aumentar no Brasil. Tal constatação alerta para a necessidade de diagnóstico precoce e intervenção clínico-cirúrgica do agravo, com o escopo de evitar mortes preveníveis. 


\section{PO 33}

\section{Ruptura traumática da aorta torácica em criança: correção cirúrgica com patch de pericardio bovino}

Luísa de Campos Guimarães e Figueiredo, João de Deus e Brito, Claudio Roberto Assumpção, Mauricio Lobo Nascimento, Eulália Thebit Pfeiffer, Lucas Moura Bezz Maciel

Introdução: As lesões traumáticas da aorta são raras na infância. Heckman e colaboradores realizaram o estudo com maior número de casos, demonstrando que estas lesões correspondem a 0,1\% do total de traumas em crianças. As lesões são mais frequentes na idade escolar e em traumas de alta energia.

Objetivo: Relatar um caso de uma criança de 2 anos com traumatismo craniano e lesão traumática da aorta torácica descendente decorrente de trauma de baixa energia, corrigidos cirurgicamente.

Métodos: Criança atendida na unidade de trauma após queda de escada. Em tomografias de crânio e abdome havia fratura de crânio e coleção hipodensa periaórtica medindo $47 \times 41 \times 35$ mm, localizada entre a $4^{\circ}$ e a 70 vértebra torácica, sugerindo aneurisma sacular de aorta, parcialmente trombosado. Após 30 dias da correção da fratura de crânio, o paciente foi submetido a toracotomia posterolateral esquerda no quarto espaço intercostal, encontrado um pseudoanerisma na aorta torácica descendente. Procedida a heparinização sistêmica, oclusão proximal e distal da aorta e abertura longitudinal da aorta, onde havia grande laceração, de natureza friável, que foi ressecada. Reconstruída a aorta longitudinalmente com patch de pericárdio bovino em sutura contínua.

Resultados: O fluxo sanguíneo foi restaurado após 27 minutos de clamp aórtico, confirmado pela pressão arterial distal normal. Pós-operatório sem intercorrências.

Conclusão: Criança com lesão aórtica traumática rara em pré-escolares e em traumas de baixa energia associada a traumatismo craniano, corrigidos cirurgicamente em dois estágios com sucesso. Após dois anos de evolução, encontra-se assintomática, sem nenhuma sequela.

\section{PO 34}

\section{Avaliação da performance e divulgação dos resultados agregando qualidade na cirurgia cardíaca}

Denise Louzada Ramos, Nilza Sandra Lasta, Viviam de Souza Ramirez, José Carlos Teixeira Garcia, Pedro Gabriel Melo de Barros e Silva, Mariana Yumi Okada, Valter Furlan

Introdução: Um dos grandes desafios na gestão hospitalar é a busca por um modelo de avaliação de desempenho das equipes médicas. Um modelo ideal deve preconizar o uso de padrões de comparação baseados em evidência, utilizando como referência as diretrizes ou metas estabelecidas.

Objetivo: Apresentar modelo de avaliação de desempenho de equipes de cirurgia cardíaca em hospital privado.

Métodos: Em 2011 elaborou-se um modelo de avaliação de desempenho das equipes cirúrgicas que se consolidou em 2012, formatado nos padrões da ética médica, baseado em diretrizes internacionais e atendendo às necessidades de qualidade da instituição. A avaliação foi dividida em três categorias de indicadores: resultados, processos e adesão a protocolos institucionais. As metas foram baseadas em indicadores internacionais e série histórica, e cada um recebeu uma pontuação de 10 a 50, totalizando 370 pontos, de acordo com a complexidade e a relevância. As equipes cirúrgicas foram notificadas e bonificadas trimestralmente, conforme o desempenho, sendo a apresentação individual e sigilosa.

Resultados: Em relação aos itens de desfecho clínico, houve diminuição dos casos de AVC, reinternação, hemotransfusão e taxa de infecção de ferida operatória. A mortalidade vem reduzindo ao longo dos anos, mas ainda não atingiu a meta estabelecida.

Conclusão: O modelo de avaliação da performance das equipes de cirurgia cardíaca demonstrou ser uma ferramenta na melhoria dos resultados e da assistência prestada, para elaborar estratégias de melhorias com envolvimento da equipe cirúrgica e dos gestores da instituição. 


\section{EXPOSIÇÃO DE PÔSTERES - SBCCV}

\section{PO 35}

\section{Redução do uso de hemoderivados em pacientes submetidos à cirurgia de revascularização do miocádio (CRM) isolada}

Denise Louzada Ramos, Nilza Sandra Lasta, Mariana Yumi Okada, Viviam de Souza Ramirez, Camila Gabrilaistis Cardoso, Pedro Gabriel Melo Barros e Silva, Valter Furlan

Introdução: A utilização de circulação extracorpórea (CEC) pode aumentar o sangramento pós-operatório (PO), contribuindo e tornando-se um dos principais fatores para a necessidade de hemoderivados. A utilização de hemoderivados relaciona-se com ocorrência de reação transfusional, infecções, aumento de morbidade e mortalidade PO, risco de imunossupressão e aumento do custo de internação hospitalar.

Objetivo: Demonstrar que uma política de redução de transfusão é efetiva, não influenciando negativamente nos desfechos clínicos.

Métodos: Estudo prospectivo, em que foram incluídos pacientes de CRM isolada entre janeiro de 2010 e outubro de 2016. Realizouse o levantamento da utilização de hemoderivados por meio da análise de relatórios do banco de sangue e prontuário médico dos pacientes. Foram comparados anualmente o número de transfusões e os desfechos: internação na UTI, internação hospitalar (Hosp), reinternação e óbito. As medidas de redução de transfusão que se iniciaram em janeiro de 2011 foram: interrupção por 5-7 dias da utilização de clopidogrel; utilização profilática de ácido épsilon-aminocaproico; adoção de novas técnicas intraoperatórias na conservação sanguínea; política institucional de incentivo à redução de transfusão. Compararam-se os períodos de préimplantação e implantação do protocolo institucional (2010 e 2011) e o do protocolo consolidado (2012 a 2016).

Resultados: Foram operados 2.028 pacientes (RMI). Houve redução do número de hemotransfusões, média de permanência na UTI, tempo médio de permanência em PO, readmissão hospitalar < 30 dias e mortalidade em 30 dias.

Conclusão: A implantação e consolidação de um protocolo institucional específico que visa ao uso criterioso de hemoderivados associado ao uso de épsilon em pacientes submetidos a cirurgia cardíaca e ações junto às equipes cirúrgica e clínica podem refletir na redução expressiva de utilização hemoderivados no PO de CRM isolada, sem interferir negativamente nos desfechos clínicos.

\section{PO 36}

\section{Valvuloplastia mitral em criança. Um desafio à população nordestina}

Euclides Martins Tenorio, Manuella Muniz, Halyna Melo, Cristina Ventura, Tereza Pinheiro, Thalita Sampaio, Marcos Gomes, Fernando Moraes Neto, Carlos Moraes

Introdução: A doença valvar mitral em crianças continua um grande desafio em nosso meio, especialmente porque a febre reumática na região ainda é um importante problema de saúde pública. Mais desafiador ainda é o fato de que um grande número dessas crianças necessita de tratamento cirúrgico nessa fase da vida.

Objetivo: Avaliar os resultados em um grupo de 232 crianças submetidas a cirurgia da valva mitral, entre 2000 e 2016, no Instituto do Coração de Pernambuco e no Instituto de Medicina Integral Professor Fernando Figueira (IMIP).

Métodos: Cento e vinte e oito (59,5\%) eram do sexo masculino e 104 (44,9\%) do sexo feminino, com idades entre 3 meses e 16 anos (média de 11 anos). Esses pacientes foram divididos em 2 grupos: grupo I, inclui 138 crianças submetidas à plastia da valva mitral e o grupo II 94, em que foi realizada troca valvar.

Resultados: A mortalidade operatória global foi de 3,8\%, sendo maior no grupo II (6 pacientes ou 6,3\%). Ao longo do período estudado, foi observado que o índice de reoperações também foi maior no grupo II (37,2\%) em comparação ao grupo I (18\%).

Conclusão: A análise da mortalidade e do índice de reoperações tem nos conduzido a fazer um esforço cada vez maior na busca pela utilização de técnicas de preservação da valva. 


\section{PO 37}

\section{Perfil dos doadores de coração do Instituto Nacional de Cardiologia}

Tamires Luciana do Nascimento Pena, Ligia Neres Matos, Viviani Christini da Silva Lima, Filipe Reis de Oliveira, Tereza Cristina Felippe Guimarães, Jacqueline Sampaio dos Santos Miranda, Bruno Miranda Marques, Vinicius Assunção Pratas Sobral, Alexandre Siciliano Colafranchesci

Introdução: O Rio de Janeiro registrando um número crescente de transplantes cardíacos. A experiência dos especialistas na seleção de doadores é fundamental para a decisão de aceitar ou não o enxerto ofertado, e há ferramentas que auxiliam no julgamento e na aceitabilidade de um doador de qualidade.

Objetivo: Descrever o perfil dos doadores de coração a partir da avaliação do Heart Donor Score (HDS).

Métodos: Estudo retrospectivo no período de 2012 a 2016. Os dados foram coletados pelo banco de dados do serviço de IC/ TX cardíaco. O HDS é aplicado em registro de transplante europeu para a avaliação de risco de doadores de coração. Este escore é utilizado na avaliação do doador e o classifica em baixo risco com pontuação menor ou igual a 16 e alto risco com pontuação maior ou igual a 17 pontos. As variáveis analisadas foram: sexo, período de internação, causa mortis, fração de ejeção (FE), sódio, uso de noradrenalina, parada cardíaca e história sorológica do doador. A análise foi descritiva em forma de frequência em percentual.

Resultados: O HDS foi aplicado em 44 doadores. O sexo prevalente foi o masculino (75\%). O período de internação variou entre 3 e 7 dias (43,1\%), sendo 36,4\%<3 dias. Trauma foi a causa mortis de maior frequência (77,3\%). A FE > 55\% foi de 79,5\% dos casos e $31,8 \%$ apresentavam nível sérico do sódio $\geq 160 \mathrm{mEq} / \mathrm{L}$. A dose de noradrenalina entre 0,1 e 0,8 mcg/kg/min foi de 38,7\% e 38,6\% sem uso. Parada cardíaca foi registrada em 9,1\% dos doadores e 6,8\% apresentaram histórico sorológico comprometido. Foram classificados como baixo risco 56,8\% e com alto risco $43,2 \%$ dos doadores avaliados.

Conclusão: O escore classificou os doadores entre baixo e alto risco, permitindo um cuidado direcionado no pós-operatório de transplante.

\section{PO 38}

\section{Transplantação cardíaca no Brasil: relevância histórica}

Dandhara Martins Rebello, Lívia Liberata Barbosa Bandeira, Carolina Orioli de Paula da Silva, Ivan Lucas Picone Borges dos Anjos, Caio Teixeira dos Santos, Thais Lemos de Souza Macedo, Ivana Picone Borges de Aragao, Antonio Rodrigues Braga Neto, Eucir Rabello

Introdução: O transplante cardíaco evoluiu muito nos últimos 30 anos. Segundo dados da International Society of Heart and Lung Transplantations, ocorreram nesse período mais de 30 mil procedimentos pelo mundo. O Brasil faz parte dessa história desde 25 de maio de 1968, 6 meses após o primeiro procedimento realizado pelo Dr. Barnards na África. A equipe do Dr. Euryclides Zerbini também se destacou ao realizar o primeiro transplante brasileiro, o 170 no mundo, embora sua equipe já se preparasse para tal feito desde antes, como demonstram trabalhos datados em 1965. A repercussão do trabalho de Zerbini foi extremamente favorável à cirurgia cardíaca brasileira, trazendo prestígio social à especialidade e beneficiando serviços em todo Brasil. O impacto do procedimento no país também gerou destaque na América Latina, tornando o Brasil referência no transplante cardíaco e guiando condutas incorporadas no mundo todo.

Objetivo: Evidenciar a importância histórica destes transplantes, utilizados até os dias atuais.

Métodos: Revisão sistemática da literatura sobre história e relevância da transplantação cardíaca no Brasil nos bancos de dados PUBMED, SCIELO e LILACS.

Resultados: Diante da atual dificuldade de realização do procedimento no Brasil pela grande demanda, os transplantes tiveram influência em caráter qualitativo, aumentando a sobrevida de pacientes com ICC em 50\%, e material, incentivando a criação do moderno centro de referência cardíaca internacional de SP, o Incor.

Conclusão: A transplantação cardíaca é um procedimento que vem sendo utilizado há anos, provando ser de grande êxito e tendo sido aprimorada e estudada no país, buscando melhor qualidade de vida e menos riscos cirúrgicos nos transplantes. 


\title{
EXPOSIÇÃO DE PÔSTERES - SBCCV
}

\section{PO 39}

\section{Correlação entre temperatura do paciente e sangramento no pós-operatório de cirurgia cardíaca com circulação extracorpórea}

\author{
Alessandra Rosalia Regla de Souza, Ari Tadeu dos Santos Lírio, Diana Fernanda Parra Vallejo
}

Introdução: O número de cirurgias cardíacas vem aumentando ao longo dos anos em virtude do aumento da longevidade da população mundial. O sangramento pós-correção cirúrgica com circulação extracorpórea (CEC) continua sendo uma das maiores intempéries no pós-operatório e um dos fatores desencadeantes deste fenômeno pode ser a baixa temperatura do paciente no momento da chegada na unidade de terapia intensiva (UTI).

Objetivo: Avaliar a correlação entre a temperatura de chegada na hora da admissão do paciente na UTI e a quantidade de sangramento nas primeiras 24 horas dos pacientes submetidos a cirurgias cardíacas com utilização de CEC.

Métodos: Trata de um estudo piloto retrospectivo, com 100 pacientes oriundos das cirurgias cardíacas realizadas no primeiro semestre de 2016 em nosso centro. Foi utilizado para análise estatística o teste de Mann-Whitney. A amostra se constituiu em 69\% de homens e 31\% de mulheres, com média de idade de 61 anos. Das cirurgias realizadas, 55\% foram revascularização do miocárdio, $24 \%$ foram trocas valvares e $21 \%$ foram outros procedimentos.

Resultados: Foi utilizada como ponto de corte a temperatura de $35,5^{\circ} \mathrm{C}$. No grupo com temperatura abaixo deste ponto 0 sangramento foi de $494 \mathrm{~mL}$, com desvio padrão de 274,7. No outro grupo, a média de sangramento foi de $505 \mathrm{~mL}$, com desvio padrão de 238,6. A análise mostrou que não houve significância estatística quanto ao sangramento no pós-operatório $(P>0,005)$.

Conclusão: Evidenciou-se que a temperatura abaixo de $35,5^{\circ} \mathrm{C}$ medida na chegada na UTI não aumenta o volume de sangramento dos pacientes submetidos a cirurgias cardiovasculares com a utilização de CEC.

\section{PO 40}

\section{Complicações abdominais no pós-operatório de cirurgia cardíaca com circulação extracorpórea}

\author{
Natasha Amin Santos Ruszczyk, Ana Letícia Varonilia Sousa
}

Introdução: As complicações abdominais no pós-operatório de cirurgia cardíaca com circulação extracorpórea são raras, com incidência de 1,2\%; porém, relacionam-se a uma mortalidade de 33\%. Para reduzir sua incidência, é necessário reconhecer os fatores de risco e aprimorar o controle hemodinâmico. Os fatores de riscos são: idade avançada, fração de ejeção reduzida, apoio inotrópico ou mecânico perioperatório, transfusões sanguíneas, arritmias, reoperações e cirurgia de emergência. A principal morbidade é a hemorragia gastrointestinal, seguida da isquemia mesentérica, pancreatite e colicistite, entre outros.

Objetivo: Explanar a respeito das complicações abdominais decorrentes do uso da circulação extracorpórea.

Métodos: Revisão bibliográfica que utilizou artigos selecionados nas bases de dados Lilacs, BVS, Scielo e livros, entre o período de 2000 a 2016.

Resultados: Para evitar tais alterações é importante maximizar o fluxo esplâncnico com a administração pré-operatória de fluidos $(1,5 \mathrm{~mL} / \mathrm{kg} / \mathrm{h}$ de cristaloides e até $600 \mathrm{~mL}$ de hetamido a 6\%), inibidores de fosfodiesterase (milrinona), descontaminação intestinal seletiva (uso pré-operatório por 3 dias de polimixina oral, tobranicina ou anfoterecina) e evitar o uso de vasopressina. Acrescenta-se, ainda, que a condução da circulação extracorpórea é essencial utilizando fluxos elevados, baixo volume de prime, manutenção do hematócrito acima de $25 \%$ e minimização de microêmbolos.

Conclusão: Conclui-se, com este trabalho, que a hipoperfusão associada a microembolia e/ou a instabilidade hemodinâmica pode afetar o trato gastrointestinal. Portanto, é imprescindível identificar os fatores de risco e manter a estabilidade hemodinâmica do paciente. 
SESSÃO

21 de abril • sexta-feira
SALA DE PÔSTERES

10h - $10 \mathrm{~h} 45$
EXPOSIÇÃO DE PÔSTERES

- SBCCV

\section{PO 41}

\section{Relação entre o perfil antropométrico e a morbidade e mortalidade na cirurgia de revascularização do miocárdio}

Helio Milani Pegado, Larissa Matos Rodrigues de Brito, Edilson Portela França Junior, Murilo Macedo Macedo, Claudio Ribeiro da Cunha, Fernando Antibas Atik, Larissa Matos Rodrigues de Brito

Introdução: O perfil antropométrico está associado com doenças crônicas e/ou mortalidade de certos procedimentos cirúrgicos em populações distintas da nossa.

Objetivo: O objetivo é avaliar, em pacientes submetidos a revascularização do miocárdio, se: 1) o perfil antropométrico influencia na escolha de enxertos, 2) o perfil antropométrico está associado a maior mortalidade e infecções em 30 dias.

Métodos: No período de janeiro de 2009 e junho de 2014, 1.787 pacientes consecutivos foram submetidos a revascularização do miocárdio isolada. O sexo masculino predominou em $67,1 \%$ e a idade média foi de $61,7 \pm 9,8$ anos. O índice de massa corporal foi calculado para todos os pacientes e adotou-se a classificação da Organização Mundial da Saúde, de baixo peso (IMC < 18,5), peso normal $(18,5-24,9)$, sobrepeso (> 25), pré-obeso $(25-29,9)$ e obeso $(>30)$.

Resultados: $O$ peso e altura médios foram $72,6 \mathrm{~kg} \pm 13,2$ e $162 \mathrm{~cm} \pm 9$, respectivamente. A maioria dos pacientes se enquadrou como sobrepeso $(45,1 \%)$, peso normal $(29,6 \%)$, obeso $(24,4 \%)$ e baixo peso $(0,9 \%)$. O perfil antropométrico não influenciou o número de enxertos utilizados (média $3 \pm 0,9$ por paciente, $\mathrm{P}=0,48$ ), nem as taxas de utilização de uma artéria torácica interna (média $98,7 \%, P=0,31$ ) ou duas (média 11,8\%, $P=0,2$ ). Não houve relação entre o perfil antropométrico e a mortalidade em 30 dias (global $2,6 \%, P=0,21)$. O grupo de obesos apresentou maior ocorrência de infecção superficial de ferida operatória $(12,1 \%, P=0,01)$ e uma tendência a mais mediastinite $(5 \%, P=0,06)$.

Discussão: Em nossa população observamos que os pacientes obesos e com sobrepeso apresentaram maior incidência de infecção superficial e profunda, sem impacto no desfecho mortalidade.

Conclusão: Não houve influência do perfil antropométrico na escolha dos enxertos. 


\section{EXPOSIÇÃO DE PÔSTERES - SBCCV}

\section{PO 42}

\section{Caracterização do perfil clínico e incidência de infecção entre pacientes submetidos a implante de dispositivo cardíaco eletrônico em um serviço de referência do Sul do Brasil}

Maria Antonieta Moraes, Jessica de Cassia M. de Almeida, Marco Antonio de Goes Victor, Tiago Luiz L. Leiria, João Ricardo Michelin Santana, Roberto T. Sant'anna

Introdução: O uso dos dispositivos cardíacos eletrônicos (DCE) aperfeiçoados tem sido uma alternativa de sucesso para salvar vidas e melhorar a qualidade de vida de pacientes com distúrbios arrítmicos.

Objetivo: Caracterizar e verificar a incidência de infecção no sitio cirúrgico de pacientes submetidos à cirurgia de implante de DCE em um serviço de referência do Rio Grande do Sul.

Métodos: Estudo de coorte retrospectiva conduzido com pacientes adultos, com idade $\geq 18$ anos, de ambos os sexos, submetidos a implante de marca-passo (MP), cardiodesfibrilador (CDI) e ressincronização cardíaca (TRC), de forma eletiva ou de urgência, de julho de 2014 a outubro de 2015. Foram analisadas as variáveis clínicas, demográficas e relacionadas aos dispositivos, além das complicações em até 30 dias após implante, extraídas de um banco de dados preexistente. Utilizou-se o teste qui-quadrado.

Resultados: Entre os 764 pacientes analisados, houve predomínio de implante de MP (76,4\%). A maioria era do sexo masculino

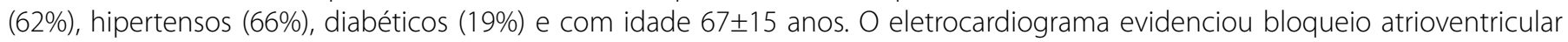
avançado em $53 \%$ e doença estrutural ausente em $64 \%$ dos pacientes. O tempo de internação foi de cinco (1-92) dias. Complicações em 30 dias ocorreram em 5,7\%: infecções sistêmicas não relacionadas ao DCE (0,5\%), infecções na incisão cirúrgica $(0,4 \%)$ e um óbito (0,2\%) por choque séptico. A hipertensão arterial sistêmica (HAS) teve associação com as complicações.

Conclusão: Verificou-se que os pacientes masculinos e sexagenários com baixa incidência de complicações em curto prazo são os mais beneficiados com os DCE nesta instituição do Sul do Brasil.

\section{PO 43}

\section{Mitral valve repair in Barlow disease}

Walter José Gomes, Alexandre R. Carvalho, Marcelo G. Silas, Nelson A. Hossne, Eduardo G. Chamlian

Introduction: Mitral valve (MV) repair is the gold standard surgical treatment for severe degenerative mitral regurgitation. Barlow syndrome is the most advanced and complex form in the spectrum of degenerative MV disease, remaining a surgical challenge owe to the extent of tissue involvement. With our growing experience in degenerative mitral valve repair, an expansion was applied for repairing Barlow disease.

Objective: To report our early experience and medium-term outcomes with MV repair in Barlow disease.

Methods: From October 2015 to June 2016, eight patients with severe MV regurgitation and anatomical features of Barlow disease (5 men and 3 women; mean age $61 \pm 8$ years) underwent MV repair, using a combination of techniques. Patients were characterized by severely enlarged MV annulus, multisegment prolapse involving both leaflets, excessive leaflet tissue, and wide regurgitant jet. Two patients had concomitant procedures (bypass grafting 1 and septal myectomy 1). Preoperatively, 1 patient was in NYHA class III, 4 in III/N and 3 in IV.

Results: The repair technique was feasible in all patients and involved leaflet resection and/or folding, chordal shortening, and complete rigid ring implantation. The most frequent prolapsed segments were P2 and A2. In a mean 5.5 \pm 3.6 months follow-up, all patients are in NYHA class I and serial echocardiogram revealed no patient with regurgitation grade > mild (four trivial e four mild).

Conclusion: The medium-term outcomes of patients with Barlow disease submitted to MV repair is satisfactory and lend support to the continued application of the technique. 


\section{PO 44}

\section{Substituição valvar aórtica na doença de Behçet - relato de caso e revisão da literatura}

Ademir Massarico Braz, Fabiano André Pereira, Thiago Vila Nova Cavalcanti de Araujo, Caio Cesar Cardoso, Celso Reis de Ávila, Tatiane Lamarca Dias, Nelson A. Hossne Jr., Eduardo G. Chamlian, Walter José Gomes

Introdução: A doença de Behçet (DB) é uma doença inflamatória crônica de origem autoimune que causa vasculite sistêmica e fragilidade tecidual. Nos casos em que aortite está presente, pode haver acometimento da valva aórtica, que geralmente se manifesta como insuficiência valvar. A substituição valvar aórtica na DB é associada com grave risco em virtude da alta incidências de deiscência de prótese (entre 40-78\%), por causa da fragilidade do ânulo aórtico induzida pela inflamação. Para reduzir esse risco, várias técnicas têm sido propostas, porem não há grandes séries de casos nesta condição.

Objetivo: Relatamos a aplicação da técnica descrita por Kotsuka para fixação da prótese no ânulo aórtico em um paciente com DB.

Métodos: Paciente masculino, 34 anos, com diagnóstico prévio de DB, foi admitido na unidade de emergência por quadro de edema agudo de pulmão. O ecocardiograma e a angiotomografia aórtica revelaram insuficiência valvar aórtica importante, dilatação e espessamento da parede da aorta ascendente. Apresentava também uveíte e lesões pustulosas em membros inferiores; o teste da patergia resultou positivo.

Resultados: Submetido a troca valvar aórtica com implante de prótese mecânica St. Jude 25, utilizando a técnica descrita por Kotsuka. Consiste no implante intravalvular da prótese, suturando a prótese valvar no ânulo aórtico ensanduichada entre os folhetos, com os pontos com pledgets passados em dupla laçada entre os folhetos e o anel aórtico inflamado. Atualmente encontra-se em acompanhamento no ambulatório especializado.

Conclusão: A substituição valvar aórtica na DB pela técnica empregada foi eficaz para evitar morbidade e mortalidade inerentemente associadas, e está recomendada para futuras intervenções em situações semelhantes.

\section{P0 45}

\section{Preoperative intra-aortic balloon counterpulsation in coronary artery bypass graft patients with severe left ventricular dysfunction}

Andrea Cristina Oliveira Freitas, Danilo Bortolotto Gurian, Wladmir Faustino Saporito, Leandro Neves Machado, Louise Horiuti, Adilson Casemiro Pires

Introduction: The intra-aortic balloon pumping (IABP) is the most used ventricular mechanical assist device. In recent years, preoperative use in patients with severe ventricular dysfunction presents itself as a great benefic strategy to the postoperative recovery.

Objective: To evaluate the IABP post-operative benefit in patients with severe ventricular dysfunction.

Methods: From January 2011 to March 2016, 125 patients underwent to a coronary artery bypass graft (CABG) with cardiopulmonary bypass and preoperative IABP in Hospital Estadual Mario Covas. The inclusion criteria were the presence of severe ischemic cardiomyopathy with left ventricular ejection fraction (LVEF) less than or equal to 40\%, estimated by Doppler echocardiography using the Simpson method. The preoperative LVEF was 30.25 $\pm 8.53 \%$, and the diastolic diameter of the left ventricle (LVDD) was $67.75 \pm 16.37 \mathrm{~mm}$. IABP was installed approximately 15 hours before the surgery.

Resultados: The patients required the IABP for $2.4 \pm 1.58$ days, and vasoactive drugs, $4.8 \pm 2.12$ days. We performed $3.2 \pm 1.9$ grafts per patient and the total length of stay was $07 \pm 5.52$ days. Cardiopulmonary bypass time was $67 \pm 10.95$ minutes and anoxia time, $46.4 \pm 10.06$ minutes. Twelve patients (9.6\%) had pneumonia and four (3.2\%), atrial fibrillation. We observed a LVDD reduction to $63 \pm 16.26(P=0.068)$ and LVEF enhancement to $36.50 \pm 16.86(P=0.144)$. The data were analyzed statistically according to the Wilcoxon test. There were no deaths.

Conclusion: The initial experience with the preoperative IABP in patients with severe left ventricular dysfunction suggests great benefit in postoperative recovery with improvement of LVEF and reduction of LVDD. 


\section{EXPOSIÇÃO DE PÔSTERES - SBCCV}

\section{PO 46}

\section{Minitoracotomia na abordagem cirúrgica da valva aórtica}

Henrique Madureira da Rocha Coutinho, Joaquim Henrique de Souza Aguiar Coutinho, João Carlos Jazbik, Ana Carolina Veltri Pacheco, Gustavo Kikuta, Bernardo Ferreira Americano do Brasil

Introdução: Durante os últimos quarenta anos a esternotomia mediana foi o acesso padrão-ouro para a abordagem cirúrgica de grande parte das doenças cardíacas. O avanço tecnológico na última década motivou a busca por cirurgias menos invasivas, que tem aumentado em popularidade em virtude de resultados estéticos satisfatórios e melhor recuperação pós-operatória.

Objetivo: Relatar nossa experiência inicial no uso da minitoracotomia para a cirurgia da valva aórtica.

Métodos: Foram estudados de forma retrospectiva 33 pacientes no período compreendido entre agosto de 2015 e setembro de 2016 que foram submetidos, em sua maioria, à miniesternotomia e em apenas um caso à minitoracotomia direita no $3^{\circ}$ espaço intercostal para troca valvar aórtica. Foram avaliadas as características pré-operatórias e todos os pacientes foram submetidos à mesma rotina de exames.

Resultados: Os tempos de CEC e pinçamento aórtico foram, respectivamente, $85 \pm 35$ min e $67 \pm 17$ min. A média de perda sanguínea pelo dreno mediastinal foi $320 \pm 330 \mathrm{~mL}$. O tempo médio de ventilação mecânica no pós-operatório foi 5,0 $\pm 3,0$ horas. 0

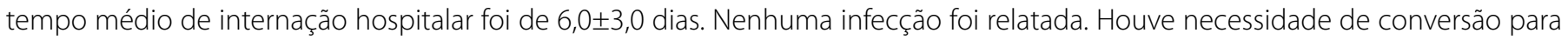
esternotomia completa em 2 casos. Dois pacientes foram a óbito em nossa casuística. A média de retorno às atividades habituais foi em torno de 21 dias após alta hospitalar.

Conclusão: A esternotomia mediana completa ainda se mantém como principal via de acesso ao coração mas acredita-se que, com o ganho de experiência das equipes cirúrgicas, a minitoracotomia ganhará cada vez mais espaço e esta técnica passará a ser realizada apenas em casos selecionados, como em cirurgias múltiplas.

\section{PO 47}

\section{Ponte miocárdica e sua correlação com a formação de placas ateroscleróticas}

Cicero Felipe Paes de Araujo Costa, Alfredo Aurélio Marinho Rosa Filho, Igor Leão Gomes Leahy, lago Moura Aguiar, Júlio César Claudino dos Santos, Lyles Regina Machado Falcão

Introdução: A ponte miocárdica (PM) é anomalia congênita das artérias coronárias, em que feixes de miocárdio envolvem um segmento de artéria coronária epicárdica, levando à compressão de um segmento desta durante a sístole ventricular. Constitui importante diagnóstico diferencial da doença arterial coronariana e pode representar fator de risco para o desenvolvimento de aterosclerose coronariana e infarto do miocárdio.

Objetivo: Correlacionar a ocorrência da ponte miocárdica à maior incidência de placas ateroscleróticas.

Métodos: Propôs-se comparar os dados com a literatura, para elucidar a associação da PM com a aterosclerose. Para isso, foi analisada uma amostra de 23 corações cadavéricos, por meio da injeção de solução salina concentrada a 0,9\% nas artérias coronárias direita e esquerda, após a lavagem abundante dos vasos e através de uma sonda de poliuretano, suturada com fio de algodão à artéria aorta, conectada a uma seringa de $50 \mathrm{~mL}$.

Resultados: A perturbação do fluxo de sangue intracoronariano, associado a elevado estresse mural proximal à PM, pode precipitar aterosclerose coronariana por lesão. Observou-se, de acordo com a literatura, uma maior proporção de acometimento aterosclerótico proximal à PM. Além disso, observam-se sinais de disfunção endotelial no segmento da PM, estimulando o vasoespasmo coronariano e a agregação plaquetária transitória, podendo levar à trombose no segmento proximal ou sob a PM.

Conclusão: A incidência de síndrome coronariana aguda aumenta nos pacientes quando a aterosclerose é sobreposta à PM. Implante de stent ou intervenção cirúrgica deve ser considerado em pacientes com os sintomas refratários ao tratamento e cujo estreitamento sistólico do segmento coronariano é significativo. 


\section{PO 48}

\section{Protocolo de demarcação de safena com utilização de ultrassom (US) para reduzir taxa de infecção em safenectomia em pacientes submetidos à cirurgia de revascularização miocárdica (CRM)}

Denise Louzada Ramos, Anna Silva Machado, Sheila Figueiredo Okada de Souza, Nilza Sandra Lasta, Paulo Henrique Herdeiro, Viviam de Souza Ramirez, Mariana Yumi Okada, Pedro Gabriel Melo de Barros e Silva, Valter Furlan

Introdução: A cirurgia de revascularização miocárdica (CRM) é uma alternativa terapêutica comum na doença coronariana grave, porém pode evoluir com complicações, especialmente infecções de sítio cirúrgico.

Objetivo: Em um esforço para reduzir a taxa de infecção em safenectomia após CRM isolada, foi desenvolvido e implementado um protocolo institucional de demarcação de safena por ultrassom em sala cirúrgica em pacientes submetidos à CRM isolada.

Métodos: Foram analisadas 1.312 CRM isoladas, de 2012 a outubro de 2016, em hospital privado brasileiro especializado em cardiologia que instituiu um protocolo de cirurgia cardíaca e participa do banco de dados da Sociedade de Cirurgiões Torácicos (STS), em que um dos itens de grande importância é redução da taxa de infecção de sítio cirúrgico. Em 2015, com o objetivo de diminuir as taxas de infecção em safenectomia, foi elaborado um protocolo de demarcação de safena por US em sala operatória pela equipe cirúrgica. No presente estudo foram criados dois grupos Grupo A - pré-instituição do US para demarcação de safena, englobando as cirurgias de 2012 a 2014, e grupo B - pós-implantação do protocolo com pacientes submetidos à CRM isolada de 2015 a outubro de 2016.

Resultados: A taxa de infecção de safena no pós-operatório foi de 7,4 (grupo A) para 2,7 (grupo B).

Conclusão: Após a implementação de mudanças na técnica de demarcação de safena e acompanhamento pós-operatório dos pacientes submetidos à CRM isolada, houve redução linear na taxa de infecção de safenectomia.

\section{PO 49}

\section{Divertículo congênito do ventrículo esquerdo como causa de tamponamento cardíaco}

Luiz Antonio Castilho Teno, Fabian Cecchi Teno Castilho, Oswaldo Teno Castilho, Thiago Rodrigues Tonello, Flavio Luis Gambi Cavallari, Daniela Sica de Araujo

Introdução: DivertículocCongênito do VE é uma condição bastante rara no adulto e as primeiras descrições se devem a óbitos por ruptura do ventrículo observadas em autópsias. Em adultos, essa malformação representa 0,4\% das autópsias em mortes de causa cardíaca e são achados casuais em 0,26\% dos pacientes submetidos a cateterismo cardíaco.

Objetivo: Relatamos o caso de uma paciente com diverticulo congênito de VE que evoluiu com tamponamento cardíaco.

Métodos: Paciente do sexo feminino, 42 anos, em tratamento para insuficiência cardíaca e investigada com ecocardiograma transtorácico. Após o exame a paciente apresentou mal súbito com síncope e foi novamente submetida a avaliação ecocardiográfica, sendo constatada a presença de derrame pericárdico moderado com restrição diastólica e tamponamento cardíaco, que não estava presente no exame anteriormente realizado. A paciente foi encaminhada para tratamento cirúrgico.

Resultados: A correção cirúrgica foi realizada com circulação extracorpórea e a paciente evoluiu bem no pós-operatório, sem necessidade de drogas vasoativas, assintomática e em boas condições.

Discussão: O divertículo congênito do VE geralmente é diagnosticado no exame rotineiro de ecocardiograma transtorácico e os diagnósticos diferenciais incluem aneurisma verdadeiro e falso aneurisma (pseudoaneurisma) do VE. Na maioria das vezes permanecem assintomáticos, porém alguns pacientes podem apresentar arritmias supraventriculares ou ventriculares, tamponamento, ruptura cardíaca e mesmo morte súbita cardíaca. Os casos de sobrevida a essas complicações são raros.

Conclusão: Sugerimos que o diverticulo congênito do VE deva ser corrigido cirurgicamente com técnicas de ressecção e sutura de baixo risco e ótimo resultado. 


\section{EXPOSIÇÃO DE PÔSTERES - SBCCV}

\section{PO 50}

\section{Precedentes relevantes da técnica cirúrgica bicaval para transplantes cardíacos ortotópicos}

Lívia Liberata Barbosa Bandeira, Carolina de Paula Orioli da Silva, Ivan Lucas Picone Borges dos Anjos, Caio Teixeira dos Santos, Dandhara Martins Rebello, Thais Lemos de Souza Macêdo, Ivana Picone Borges de Aragão, Eucir Rabello, Antonio Rodrigues Braga Neto, Renan da Rocha Soares, Patricia Rangel Sobral Dantas

Introdução: A alta taxa de mortalidade relacionada ao transplante cardíaco foi a precursora para o desenvolvimento de novas técnicas que possibilitaram o aprimoramento do procedimento ao redor do mundo; dentre elas, a técnica bicaval para transplantes cardíacos ortotópicos, que vem sendo utilizada há 30 anos com eficácia em razão da facilidade técnica e do menor índice de complicação pós-operatória.

Objetivo: Evidenciar a importância histórica da técnica cirúrgica bicaval para transplantes cardíacos ortotópicos com seus precedentes resultados.

Métodos: Foram utilizados banco de dados como PubMed, SBC e SciELO.

Resultados: De acordo com a literatura, foi realizado um estudo com 29 pacientes em grau funcional IV da New York Heart Association e em uso de medicação em dose maximizada, com pouca resposta à terapêutica clínica e cuja expectativa de vida era inferior a 12 meses, dos quais 22 foram operados utilizando-se a técnica ortotópica clássica, 6 pela bicaval e 1 por técnica heterotópica, e todos foram submetidos a esquema com corticosteroides, biópsias endomiocárdicas e ecocardiogramas periódicos. Os resultados evidenciaram uma mortalidade de 13,79\% em 30 dias, e tais pacientes haviam sido operados pela técnica clássica; os 6 pacientes operados pela técnica bicaval permaneceram vivos por um período de 12 a 15 meses após a cirurgia.

Conclusão: $O$ transplante cardíaco pela técnica bicaval categorizada como simples provou ser de êxito em virtude da diminuição das cavidades atriais, com baixa incidência da necessidade do uso de marca-passo após a cirurgia e menor ocorrência de regurgitação atrioventricular.

\section{PO 51}

\section{Cirurgia cardíaca por mini-incisão com circulação extracorpórea. Resultados iniciais de 106 casos consecutivos operados em nosso serviço}

Ricardo Barros Corso, Isaac Azevedo Silva, Helmgton José Brito de Souza, Glauco Kalil da Silva Pina, Marcus Vinicius Nascimento dos Santos

Objetivo: Descrever nossa experiência inicial e consecutiva de 106 pacientes operados com técnicas mini-invasivas e os resultados imediatos observados.

Métodos: São descritos 106 pacientes consecutivos submetidos à cirurgia cardíaca por mini-incisão em nosso serviço, operados entre outubro de 2010 e novembro de 2016. Utilizou-se a miniesternotomia superior em J ou a minitoracotomia direita. A videoassistência foi utilizada para as operações da valva mitral. Os vasos femorais foram canulados para a instalação da circulação extracorpórea (CEC) na maioria dos pacientes. Idade avançada, hipertensão arterial pulmonar acima de $60 \mathrm{mmHg}$ e necessidade de revascularização miocárdica concomitante foram nossos critérios de exclusão. Cinquenta e cinco pacientes (51,9\%) eram do sexo masculino, média de idade de 53,5 anos. Euroscore II médio foi de 1,93\%. Realizaram-se: 40 trocas de valva aórtica, 27 trocas da valva mitral, 20 plastias da valva mitral, 9 correções de comunicação interatrial, 4 operações de Bentall de Bonno, 3 correções de comunicação interventricular, 2 correções de aneurisma de aorta ascendente e troca aórtica, 1 correção de pseudoaneurisma de ventrículo esquerdo. Um paciente necessitou de conversão para estenotomia mediana.

Resultados: Não houve óbito operatório. Houve 5 (4,7\%) óbitos hospitalares.

Conclusão: As operações cardíacas por mini-incisão implicam treinamento específico com as novas técnicas operatórias, necessidade de instrumentais, insumos e de equipamentos dedicados e uma nova curva de aprendizado ao cirurgião cardiovascular. As técnicas por mini-incisão possuem vantagens na abreviação da recuperação pós-operatória e no resultado cosmético para os pacientes. 


\section{PO 52}

\section{Reparo valvar mitral: análise a longo prazo dos resultados clinicos e ecocardiográficos \\ Rodrigo Coelho Segalote, Clara Weksler, Laila Lubiana Maciel, Lara Borges Ribeiro, Andre Luis de Albuquerque Magioli, Leandro Passarelli Barbosa, Laura Rangel Duncan, Thamires de Almeida Marcon, Bruno Marques}

Introdução: A preservação da valva mitral tem se mostrado superior à cirurgia de troca mitral no paciente não isquêmico.

Objetivo: Apresentar os resultados da cirurgia de reparo mitral no paciente não isquêmico no Instituto Nacional de Cardiologia (INC), por meio da análise dos resultados imediatos e da avaliação ecocardiográfica imediata e a longo prazo.

Métodos: Estudo observacional, com 161 pacientes operados de 2008 a 2016, para reparo mitral isolado ou combinado (cirurgia valvar, revascularização miocárdica, correção de arritmias e cardiopatias congênitas), em casos de doença valvar mitral degenerativa, reumática e congênita, insuficiente e/ou estenótica. Foram analisadas a mortalidade operatória, as características da doença valvar mitral, os tipos de técnicas empregadas e os resultados ecocardiográficos imediatos e tardios. O tempo de seguimento médio foi de $42,1 \pm 28,5$ meses.

Resultados: Foram operados 161 pacientes (76 feminino/85 masculino) com idade média de 50,5+/-17,3 anos (94 degenerativos, 25 reumáticos, 16 congênitos e 7 funcionais). Sessenta e seis sofreram reparo mitral isolado, 83 foram cirurgias combinadas e 13, cirurgias de cardiopatias congênitas. A mortalidade operatória foi de 8 pacientes, sendo 1 no reparo mitral isolado. Cinco pacientes necessitaram de troca valvar. A avaliação ecocardiográfica imediata mostrou insuficiência discreta/leve em 90,9\%; leve/moderada em 2,2\%; moderada em 5,6\%; grave em 1,1\%. A avaliação tardia mostrou insuficiência discreta/leve em 79,1\%; leve/moderada em 6,9\%; moderada em 9,7\%; moderada/grave em 4,1\% dos pacientes. Três pacientes foram reoperados tardiamente, 1 para re-reparo e 2 para troca valvar.

Conclusão: O reparo mitral no paciente não isquêmico no INC apresenta resultados clínicos e ecocardiográficos satisfatórios imediatos e a longo prazo.

\section{PO 53}

\section{Protocolo de alta precoce em pacientes submetidos à cirurgia de revascularização do miocárdio (CRM)}

Denise Louzada Ramos, Nilza Sandra Lasta, Viviam de Souza Ramirez, Mariana Yumi Okada, Camila Gabrilaitis Cardoso, Antonio Claudio do Amaral Baruzzi, Gianni Manzo, José Carlos Teixeira Garcia, Pedro Gabriel Melo Barros e Silva, Valter Furlan

Introdução: A preocupação com o tempo de internação do paciente em pós-operatório de cirurgia cardíaca tem sido crescente, tanto pelas complicações como pela tentativa na redução do custo global dos procedimentos.

Objetivo: Avaliar a segurança de um protocolo de alta precoce em pacientes submetidos à CRM isolada.

Métodos: Foram incluídos pacientes submetidos à CMR isolada entre janeiro de 2012 e outubro de 2016, sendo 2012 foi o período pré-protocolo, 2013 o período de implementação e 2014 a 2016 o período do protocolo consolidado. Foram divididos dois grupos e comparados: A - período pré-protocolo e implementação e B - pós-consolidação do protocolo. Analisaram-se: tempo médio de intubação, alta da UTI no 10 PO, alta hospitalar no 40 PO, tempos médios de internação em UTI e hospitalar, reinternação em UTI e hospitalar, mortalidade. O protocolo consiste na avaliação do nível de sedação, aumento da frequência de avaliação fisioterapêutica para desmame precoce e reuniões com as equipes para divulgação dos resultados.

Resultados: Um total de 1.372 pacientes foram submetidos à CRM isolada no período analisado.

Conclusão: Observou-se que, na consolidação do protocolo de alta precoce, obteve-se redução no tempo de intubação orotraqueal, aumento nas taxas de alta de UTI no $1^{\circ}$ PO e hospitalar no 40 PO, culminando na redução nos tempos de permanência pósoperatória. Apesar da redução da permanência, não houve aumento nas taxas de reinternação e taxa de mortalidade, sugerindo que para pacientes selecionados, após avaliação criteriosa, esta prática pode ser utilizada rotineiramente. 


\section{EXPOSIÇÃO DE PÔSTERES - SBCCV}

\section{PO 54}

\section{Protocolo padronizado para reduzir mediastinite após cirurgia cardíaca: uma iniciativa de melhoria de qualidade de um hospital privado brasileiro}

Denise Louzada Ramos, Anna Silva Machado, Sheila Figueiredo Okada de Souza, Nilza Sandra Lasta, Paulo Henrique Herdeiro, Mariana Yumi Okada, Pedro Gabriel Melo de Barros e Silva, Valter Furlan

Introdução: A mediastinite é uma grave complicação infecciosa do pós-operatório (PO) de cirurgia cardíaca, implicando desfechos desfavoráveis ao paciente.

Objetivo: Reduzir casos de mediastinite em pacientes submetidos à cirurgia de revascularização do miocárdio (CRM) após consolidação de protocolo institucional.

Métodos: Desde 2011, um hospital cardiológico participa do banco de dados da Sociedade de Cirurgiões Torácicos (STS). Os primeiros relatos evidenciaram maior número de casos de mediastinite em comparação com hospitais americanos. Foi desenvolvido protocolo para reduzir incidência de mediastinite. O protocolo foi aplicado aos pacientes submetidos à CRM a partir de março de 2012 e consistiu em abordagem pré e pós-operatória. Na avaliação pré-operatória é entregue um kit com clorexidina 2\% para banho, mupirocina $20 \mathrm{mg} / \mathrm{g}$ para uso tópico e clorexidina 0,12\% para a higiene oral e o paciente instruído a realizar estas medidas, durante 5 dias antes da cirurgia. Foi adicionado um antimicrobiano glicopéptido na profilaxia em casos específicos. Também foi implementado um rigoroso controle de glicemia perioperatória. Na consulta de acompanhamento após a cirurgia foi realizado diagnóstico precoce de infecção.

Resultados: A ocorrência de mediastinite pré-protocolo (de janeiro de 2011 a março de 2012) foi de 2,10\% (9/429). Durante o período de execução ocorreram 2 casos em 195 cirurgias (1,03\%) e após consolidação do protocolo (outubro de 2012 até outubro de 2016), a taxa foi de 0,09\% (1/1075 CRM).

Conclusão: Após a implementação de mudanças práticas baseadas em evidências, uma redução linear na taxa de mediastinite foi documentada e sustentada.

\section{PO 55}

\section{Dominância arterial coronariana aplicada ao estudo do infarto agudo do miocárdio}

Alfredo Aurélio Marinho Rosa Filho, Igor Leão Gomes Leahy, Lyles Regina Machado Falcão, Julio Cesar Claudino dos Santos, lago Moura Aguiar, Cicero Felipe Paes de Araújo Costa

Introdução: O conhecimento da dominância arterial coronariana em pacientes com infarto agudo do miocárdio é um recurso anatômico importante que possibilita ao profissional discriminar sobre a melhor terapêutica nesse tipo de cardiopatia isquêmica.

Objetivo: Verificar a incidência da dominância coronariana e o número de ramos que as artérias coronárias emitem para o ventrículo oposto em peças cadavéricas humanas existentes no Laboratório de Anatomia da Universidade Estadual de Ciências da Saúde de Alagoas (UNCISAL).

Métodos: Foram utilizados 23 corações íntegros, excluindo-se aqueles com lesões, cicatrizes ou dissecados. Após a lavagem abundante dos vasos para remoção de sangue, coágulos ou trombos, foram injetados, com uma seringa, $50 \mathrm{~mL}$ de solução salina em uma sonda de poliuretano suturada com fio de algodão à artéria aorta. Concluída a instilação, foram observados os ramos intumescidos com a solução salina e realizada a contagem dos ramos principais e segmentares para a determinação da dominância coronariana.

Resultados: Pôde-se verificar que a dominância direita ocorreu em 17 peças, aproximadamente 74\%, das quais 67\% apresentaram 1 ramo para o ventrículo esquerdo e 33\% apresentaram 2 ramos, enquanto a dominância esquerda esteve presente em 4 peças, aproximadamente $17 \%$, das quais $74 \%$ apresentaram 2 ramos para o ventrículo direito, $11 \%$ apresentaram 1 ramo, $11 \%$ apresentaram 3 ramos, 5\% apresentaram 4 ramos e a codominância foi observada em 2 peças, aproximadamente 9\%.

Conclusão: O mais prevalente foi a dominância coronariana direita com a emissão de 1 ramo para o ventrículo esquerdo. 


\section{PO 56}

\section{Análise da topografia das pontes miocárdicas em cadáveres aplicada ao diagnóstico de doença arterial coronariana}

lago Moura Aguiar, Alfredo Aurélio Marinho Rosa Filho, Cicero Felipe Paes de Araujo Costa, Igor Leão Gomes Leahy, Lyles Regina Machado Falcão, Júlio César Claudino dos Santos

Introdução: A ponte miocárdica (PM) é uma anomalia congênita das artérias coronárias em que alguns feixes de miocárdio cruzam ou envolvem determinada parte da artéria coronária que se dispõe no epicárdio. Ela constitui um dos principais diagnósticos de doença arterial coronariana, podendo mostrar-se como angina pectoris, isquemia miocárdica e, menos comum, infarto agudo do miocárdio (IAM) ou morte súbita.

Objetivo: Por meio do estudo em peças cadavéricas, observar o número de ramos arteriais mais acometidos na anomalia estudada e avaliar quais dos ramos existentes são mais frequentes.

Métodos: Propôs-se comparar os dados com a literatura atual, a fim de elucidar o perfil clínico e o prognóstico de pacientes com ponte miocárdica. Para isso, foi analisada uma amostra de 23 corações cadavéricos, por meio da injeção de solução salina concentrada a 0,9\% nas artérias coronárias direita e esquerda, após a lavagem abundante dos vasos e através de uma sonda de poliuretano, suturada com fio de algodão à artéria aorta, conectada a uma seringa de $50 \mathrm{~mL}$.

Resultados: Terminado o procedimento, constatou-se o fenômeno da PM em 75\% dos corações, onde o ramo mais acometido foi o interventricular esquerdo, seguido pelo interventricular direito. Todos os corações apresentaram o acometimento de somente uma artéria coronária, sendo a artéria interventricular esquerda acometida em 73,3\% dos casos, prevalecendo em seu terço médio.

Conclusão: Diante de sua importância, faz-se necessário o maior conhecimento anatômico dessa doença, permitindo um melhor diagnóstico.

\section{PO 57}

\section{Aneurisma de artéria coronária com fístula complexa para artéria pulmonar}

Jeú Delmondes de Carvalho Junior, Cristiano Berardo, Diogo Ferraz, Fernando Augusto Figueira, Rodrigo Tchaik, Edmilson Cardoso, João Paulo Segundo, Felipe Ribeiro Walter

Introdução: Aneurisma gigante da artéria coronária esquerda com trajeto fistuloso complexo é extremamente incomum e raro. Não encontramos na literatura relatos de fístula aneurismática do tronco da coronária esquerda para a artéria pulmonar.

Objetivo: Relatar caso de dor torácica típica associada à dispneia, em paciente com aneurisma de artéria coronária com trajeto fistuloso para a artéria pulmonar.

Métodos: O diagnóstico foi realizado após estratificação para doença arterial coronariana. O cateterismo evidenciou imagem de shunt esquerda-direita do tronco da coronária esquerda para a artéria pulmonar. O diagnóstico foi complementado com uma angiotomografia.

Discussão: A abordagem cirúrgica foi escolhida em razão da complexidade e multiplicidade do trajeto fistuloso. Nesse caso, não houve acometimento do leito nativo da artéria coronária, sendo desnecessário realizar qualquer tipo de revascularização.

Resultados: Foi realizada identificação da extremidade distal do trajeto fistuloso após abertura e inspeção da artéria pulmonar. Realizada sutura do óstio distal da fístula pela face interna da artéria pulmonar, ressecção do trajeto fistuloso e fechamento proximal com clipe metálico e sutura. A paciente evoluiu assintomática, sem complicações no pós-operatório.

Conclusão: Aneurismas de artérias coronárias são achados eventuais e extremamente raros, geralmente relacionados a vasculites, como doença de Kawasaki. Aneurismas gigantes, com trajeto fistuloso e idiopáticos, são ainda mais incomuns e complexos, exigindo tratamento individualizado, objetivando exclusão da fístula mantendo perfusão miocárdica. O tratamento pode ser cirúrgico ou percutâneo com embolização, a depender da anatomia coronariana e sua relação com o aneurisma e a fístula. 


\section{EXPOSIÇÃO DE PÔSTERES - SBCCV}

\section{P0 58}

\section{Perfil clínico e cirúrgico e resultado de um ambulatório de feridas operatórias em pacientes submetidos a cirurgia cardiovascular}

Maria Antonieta Moraes, Elizabete Rosane Palharini Yoneda Kahl, Renata da Costa Brião, Luciane Raminelli da Silveira, Jéssica de Cássia Marques de Almeida, Pauline Eloíse Mariani

Introdução: Infecções de sítio cirúrgico (ISC) pós-operatórias de cirurgia cardíaca são complicações comuns na prática clínica. A literatura aponta elevada incidência de infecções em incisões esternais e mediastinites causadas por diferentes fatores preditores.

Objetivo: Verificar o perfil clínico, cirúrgico e terapêutico de um ambulatório de tratamento de feridas operatórias em pacientes submetidos a cirurgia cardiovascular.

Métodos: Estudo de coorte histórica conduzido com pacientes submetidos a cirurgia cardiovascular e acompanhados em um ambulatório de feridas, entre abril de 2015 a abril de 2016. Coleta de dados realizada a partir dos prontuários. Buscou-se identificar as variáveis clínicas, cirúrgicas, microrganismos predominantes, antibioticoterapia prescrita, produtos utilizados nos curativos e tempo de acompanhamento ambulatorial. Os desfechos avaliados foram desenvolvimento de ISC e mediastinite.

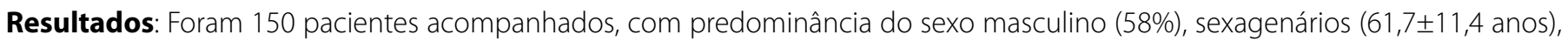
hipertensos (75\%), diabéticos (44,7\%). Cirurgias prevalentes: revascularização do miocárdio (79,3\%) e valvar (17,3\%). Necessidade de reinternações (25,3\%) e reintervenções cirúrgicas (26,7\%). ISC: safenectomia (17\%) e torácicas (16\%). Mediastinite (8\%) e ISC (29,3\%), sendo incisionais (17\%), profundas (7\%) e cavidade/órgão (6\%). Microrganismos predominantes: Serratia sp (6,7\%) e KPC (4,7\%). Antibióticos prevalentes: cefepima (52,3\%), piperacilina+tazocin (36,4\%), meropenem (34,1\%). Associação entre ISC e diabetes mellitus $(P=0,000)$. Produtos mais utilizados para os curativos: ácidos graxos $(80 \%)$ e alginato de cálcio (19,3\%). Tempo médio de acompanhamento ambulatorial: $35 \pm 71$ dias.

Conclusão: Pacientes do sexo masculino, sexagenários, hipertensos, diabéticos e revascularizados constituem a população deste ambulatório de feridas. O acompanhamento especializado e a realização de curativos com produtos específicos na ferida operatória propiciam uma cicatrização eficaz.

\section{PO 59}

\section{Experiências e estratégias de um serviço que adotou a revascularização do miocárdio com enxertos arteriais}

Andreza Sérvula Pereira da Silva, Samuel Soares Eduardo, Paulo Marcelo Barbosa Mesquita

Introdução: A utilização de enxertos arteriais iniciou-se com o uso da artéria torácica interna esquerda (ATIE). Há evidências de que o acréscimo de outro enxerto arterial ao tratamento pode diminuir a morbidade e a mortalidade a longo prazo. Como opções para segundo enxerto arterial, podemos citar a artéria torácica interna direita (ATID) e artéria radial.

Objetivo: Avaliar a aplicabilidade dos enxertos arteriais múltiplos.

Métodos: Estudo retrospectivo. Consiste na coleta de dados de prontuários dos pacientes submetidos à cirurgia de revascularização do miocárdio, no período de janeiro de 2013 a outubro de 2016 no serviço de referência em cirurgia cardíaca no interior do Ceará. Os enxertos arteriais utilizados foram ATIE, ATID e artéria radial.

Resultados: Nos 332 pacientes, 800 coronárias revascularizadas, utilizando-se ATIE em 100\% dos casos, ATID em 114 deles e artéria radial em 35. Considerando-se apenas as cirurgias com dois ou mais vasos revascularizados, contabilizamos 271 pacientes, 739 anastomoses, com média de 2,7 coronárias por cirurgia. A ATID foi a segunda opção em 114 casos e a radial, em 35. Em 102 das cirurgias, foi utilizado exclusivamente enxerto arterial. A configuração de enxerto composto em Y foi utilizada em 29,5\%, em I em $8,5 \%$ e o sequencial, em 24,3\%.

Conclusão: O uso das artérias torácicas internas bilaterais e da artéria radial, nas suas variadas configurações, é uma importante estratégia. Esses artifícios devem ser utilizados visando aumentar o arsenal para revascularizar as coronárias com enxertos arteriais. 


\section{PO 60}

\section{Correção robô-assistida da persistência da veia cava superior esquerda drenando em átrio esquerdo}

Carlos Eduardo Tossuniam, Paola Keese Montanhesi, Allison Parilla Toschi, Gustavo Foronda, Diná Mie Hatanaka, Gabriel Franzin Rusca, Alexandre Teruya, Cesar Nomura, Robinson Poffo

Introdução: A persistência da veia cava superior esquerda (VCSE) é a alteração mais comum do retorno venoso sistêmico do coração. Resulta da falha na involução da veia cardinal anterior esquerda. Sua incidência varia de 0,3 a 0,5\% na população normal e 4,3 a 5\% nos pacientes com cardiopatia congênita. A veia inominada está ausente em $65 \%$ dos casos. A VCSE persistente drena em 80 a 90\% dos casos no átrio direito pelo seio coronariano e no restante dos casos, no átrio esquerdo.

Objetivo: Descrever novo método para correção cirúrgica da persistência da VCSE.

Métodos: Paciente do sexo feminino, 32 anos, com dispneia aos mínimos esforços. Exame físico mostrava grande hepatomegalia, estase de veias jugulares e discreto edema de membros inferiores. O ecocardiograma demonstrava VCSE conectada ao AE com fluxo E-D; VCS direita presente; dilatação importante do AD e VD, com AE e VE normais; hiperfluxo pulmonar importante e função biventricular preservada. A angioTC acrescentou drenagem da veia hemiázigos na VCSE e presença de veia inominada A cirurgia foi realizada com intubação seletiva; trocarte $12 \mathrm{~mm}$ introduzido no 30 espaço intercostal esquerdo para a câmera; 3 trocartes $8 \mathrm{~mm}$ foram utilizados para os demais instrumentos. Circulação extracorpórea foi instituída por canulação dos vasos femorais. VCSE e veia hemiázigos foram ligadas com suturas de PTFE.

Resultados: Sem complicações durante o procedimento, sem necessidade de toracotomia, excelente recuperação pós-operatória, excelente resultado estético. Alta no 40 PO.

Conclusão: A correção robô-assistida da persistência da VCSE parece ser um método seguro e eficaz.

\section{PO 61}

\section{Correção endovascular de pseudoaneurisma de aorta ascendente após cirurgia carecia prévia, por via transapical}

Lucas Regatieri Barbieri, Oscar J. M. Medranos, Thais A. P. P. Couto, Eliane Cruz, Gilmar G. Santos, Victor Hadad, Noedir A. G. Stolf

Introdução: Uma complicação comum que tem sido relatada após cirurgias cardiovasculares é o pseudoaneurisma da aorta (PSAs). A segurança e eficácia das terapêuticas endovasculares para esta condição ainda são controversas. No entanto, a cirurgia de PSAs está associada com altas mortalidade e morbidade. O tratamento percutâneo tem sido descrito com alternativa.

Métodos: Paciente de 67 anos, sexo feminino, com uma disfunção de bioprótese aórtica. Foi reoperada, com implantação de nova bioprótese. A paciente evoluiu sem complicações. Readmitida com sangramento através de incisão esternal (pot). A AngioTC revelou um pseudoaneurisma da aorta ascendente distal. Reoperada com hipotermia e parada circulatória, por técnica convencional aberta. No 70 PO, a paciente apresentou evento similarconfirmado por ANgioTC. Optou-se por correção endovascular. Endoprótese Gore-CTAG foi introduzida através do ápice do ventrículo esquerdo e foi posicionada com o auxílio de angiografia e ecocardiograma transesofágico.

Resultados: A angiografia de controle mostrou excelente resultado. A paciente teve uma recuperação pós-operatória excelente e recebeu alta hospitalar no 70 dia. Um mês depois, a AngioTC revelou oclusão completa do pseudoaneurisma.

Conclusão: A incidência de PSA aórtico tem sido relatada para ser tão baixa quanto 0,5\%. Entretanto, maiores incidências foram relatadas em pacientes após cirurgia cardíaca ou aórtica. Em até $60 \%$ das cirurgias aórticas, os PSAs aórticos ocorrem ao nível da linha de sutura após a cirurgia. Procedimentos de raiz aórtica, como o de Bentall, são os mais comumente associados, representando 55\% dos casos. O primeiro relato de um método percutâneo para o fechamento de um PSA ascendente foi em 2005. 


\section{EXPOSIÇÃO DE PÔSTERES - SBCCV}

\section{P0 62}

\section{Aneurisma do seio de Valsalva com fístula aorta-átrio direito: relato de um caso raro com importante repercussão hemodinâmica}

Vanessa Kessia Silva Teixeira de Moura, João Victor Campos da Silva, Bruna Gomes de Castro, Marina Mendes Melo, Gustavo Maffra Monteiro, Vitória Mikaelly da Silva Gomes, Vera Nascimento Gomes Victória, Josinaldo Santos da Costa, Kamilla Peixoto Bandeira, Igor Medeiros Sena, Peter Conde Vidal Júnior, Laio Caju Wanderley, Rafaela da Hora Sales, Francisco Siosney Almeida Pinto, José Wanderley Neto

Introdução: Aneurisma do seio de Valsalva (ASV), doença rara, possui etiologia sobretudo congênita, relacionada com fraqueza na junção da camada média da aorta e ânulo-aórtica. Os sintomas aparecem na fase adulta, expressos por dor torácica, dispneia aos esforços e palpitações. O ASV pode fistular ou romper-se para a parede ou cavidades cardíacas. Entre suas complicações está a fístula com o pericárdio, resultando em tamponamento cardíaco.

Objetivo: Relatar caso de paciente com aneurisma do seio de Valsalva com fístula aorta-átrio direito e importante repercussão hemodinâmica.

Métodos: Estudo descritivo, observacional, de relato de caso de aneurisma do seio de Valsalva com fístula aorta-átrio direito.

Resultados: Paciente, sexo masculino, 20 anos, com dispneia e palpitações pouco frequentes, NYHA ll. Encaminhado a Santa Casa de Misericórdia de Maceió com ecocardiograma revelando aneurisma de seio de Valsalva com fistulização e drenagem para interior do átrio direito, dilatação importante de ventrículo esquerdo e moderada de aurícula direita. Cineangiocoronariografia mostrou fluxo contínuo do seio de Valsalva não coronariano para o átrio direito, confirmando presença de fístula. A correção foi feita por meio do fechamento da fístula com fio Prolene 4-0 e pericárdio bovino.

Conclusão: Com amplo espectro clínico, apresenta-se normalmente roto e sintomático, facilitando o diagnóstico. O fechamento da fístula aorta-átrio direito é a melhor opção para maioria dos casos. O uso do enxerto de pericárdio bovino é recomendado visando evitar tensão do tecido remanescente do seio de Valsalva e deformações do anel aórtico, diminuindo recorrência de fístula e incidência de insuficiência aórtica, mais frequentes no fechamento direto.

\section{PO 63}

Impacto do uso de fio de poliglactina impregnado com triclosan na prevenção de infecção de ferida de safenectomia em cirurgia de revascularização do miocárdio: ensaio clínico prospectivo, duplo-cego e randomizado

Paulo Samuel Santos Filho, Bernardo Rangel Tura, Marisa Santos, Alexandre Siciliano Colafranceschi, Andréa Nunes de Souza Pragana, Marcio Lassance, Mateus Swarosky, Marcelo Goulart Correia, Heloisa Helena Simões, Ana Paula Malta S. Santos, Rafael Quaresma Garrido, Márcia Regina Vasques, Kátia Marie Sena, Paulo Eugênio S. Figueiredo, Rafael Louzeiro, Marcia Bellotti, José Oscar Brito Reis, Bruno Miranda Marques

Introdução: As infecções de sítio cirúrgico (ISC) apresentam significativas morbidade e mortalidade. As ISC das feridas de safenectomias, nas eevascularizações do miocárdio (RM), são de 2 a 20\% em diferentes estudos, e aumentam o tempo de internação e os custos hospitalares.

Objetivo: A incidência de infecção nas feridas de safenectomia nas RM foi de 10\% no ano de 2009 e nosso objetivo é testar a intervenção para reduzi-la.

Métodos: De fevereiro de 2011 a junho de 2014, realizou-se este ensaio clínico, duplo-cego e randomizado, operando 508 pacientes exclusivamente de RM, com ou sem circulação extracorpórea (CEC). Usamos o fio de poliglactina comum (PC) em 257 pacientes controle e impregnado com triclosan (PT) em 251 pacientes intervenção, nas sínteses das feridas das safenectomias dos operados no INC.Como hipótese, sugerimos que o uso de suturas impregnadas por triclosan reduziriam a incidência de ISC das safenectomias. OTriclosan é uma substância antibacteriana que reduz o crescimento de bactérias, por inibição da síntese dos ácidos graxos. Os pacientes tiveram avaliações em torno do sétimo, décimo-quinto e trigésimo dia após a cirurgia. Nestes dias eles tiveram suas suturas examinadas, avaliadas para dor e temperatura e fotografadas e, foram realizados exames laboratoriais.

Resultados: O desfecho primário mostrou 13 infecções (5,3\%) nas feridas suturadas com PT e 20 infecções (7,9\%) nas suturadas com PC ( $P=0,294$ Dos 508 pacientes, 204 eram diabéticos sendo que 50,6\% usaram PC e 49,4\%, PT. Tiveram dor nas feridas 47 dos 257 pacientes suturados com PC (18,22\%) e, 25 dos 251 suturados com PT (9,92\%)-Valor de $P=0,00759$. A temperatura estava aumentada nas feridas de 14 dos 257 pacientes suturados com PC (5,43\%) e, de 4 dos 251 suturados com PT (1,59\%). - Valor de P=0,0282.

Conclusão: $O$ fechamento de pernas e coxas em pacientes operados de RM com suturas de poliglactina impregnadas por triclosan reduz a incidência de ISC das feridas de safenectomias, apesar de não haver significância estatística. Houve redução na dor e na hipertermia das feridas. 


\section{PO 64}

\section{Resultado de transplante cardíaco pediátrico em paciente menor de 1 ano}

Camylla Santos de Souza, Bruna Gomes de Castro, Matheus Henrique Seixas dos Santos, Maria Carolina Nogueira Trompieri, Bianca Alves de Miranda, Caroline Sbardellotto Cagliari, Patrícia Fraga Paiva, Mateus Francelino Silva, Thamires Politano De Sant'Anna Alves, Matheus Jorge Pires Viana, Alice Albuquerque Figueiredo, Guilherme Nobre Cavalcanti Lucas, Victória Queiroz Ramos, João David de Souza Neto, Daniel Francisco de Mendonça Trompieri, Juan Alberto Cosquillo Mejia, Valdester Cavalcante Pinto Júnior

Introdução: Nas cardiopatias congênitas (CC), o transplante cardíaco pediátrico é considerado quando a expectativa de sobrevida é inferior a dois anos, quando estão esgotadas as terapêuticas conservadoras, e quando existe uma qualidade de vida inaceitável. Entre as causas de CC que preconizam transplante, está a citomegalovirose (CMV), infecção congênita mais comum (0,2 a 3\% dos nascimentos). Apesar disso, a realização do procedimento é naturalmente rara, pelo número pequeno de doadores e pelas particularidades anatômicas.

Objetivo: Estudar caso de transplante cardíaco em paciente menor de 1 ano com CMV congênita.

Métodos: Análise do prontuário de paciente no Hospital de Messejana (HM), em Fortaleza, e revisão bibliográfica nas bases de dados Pubmed, Medline e SciELO.

Resultados: A.V.R.S., sexo feminino, 3 meses de idade, nascida pré-termo, com síndrome hipoplásica do coração esquerdo, aorta de $2,8 \mathrm{~mm}$ e insuficiência tricúspide importante. Sorologia positiva para CMV e negativa para toxoplasmose. Apresentou Klebsiella à hemocultura, tratada com cefepime. Foi submetida a transplante cardíaco ortotópico no HM, com doador também positivo para CMV. No pós-operatório imediato apresentava-se em grave estado geral, anasarcada, intubada em ventilação mecânica, com tempo de enchimento capilar <2", sedada e curarizada. Evoluiu com melhora do quadro clínico. Ao nono dia pós-operatório, apresentou, ao ecocardiograma, leve regurgitação tricuspídea, PSAP $35 \mathrm{mmHg}$, função sistólica biventricular preservada, fração de ejeção de 73\%, ausência de derrame pericárdico e de ectopias. Segue internada, estável, evoluindo com melhora clínica.

Conclusão: O transplante cardíaco pediátrico possibilita maior sobrevivência e melhora da qualidade de vida em portadores de CC, sendo fundamental superar as adversidades envolvidas na realização do procedimento.

\section{PO 65}

\section{Nano e microtopografias direcionais influenciam opostamente a diferenciação de células-tronco derivadas de tecido adiposo em células musculares lisas: novas perspectivas para a otimização de biomateriais para a engenharia de tecidos vasculares}

Gabriel Romero Liguori, Guilherme Garcia Barros, Qihui Zhou, Philipp Till Kuhn, Patrick Van Rijn, Martin Conrad Harmsen, Luiz Felipe Pinho Moreira

Introdução: A engenharia de tecidos consiste em uma promissora alternativa para o desenvolvimento de enxertos vasculares. Topografias direcionais em biomateriais influenciam a adesão, o alinhamento e a diferenciação das células tronco. Até o presente momento, pouco é sabido sobre como topografias lineares poderiam ser exploradas para direcionar o comportamento celular visando otimizar biomateriais para gerar vasos sanguíneos por meio da engenharia de tecidos.

Objetivo: Investigar a resposta de células-tronco derivadas de tecido adiposo (ASCs) quando colocadas em gradientes de diferentes tamanhos de topografia direcional, variando unidirecionalmente o comprimento da onda ( $\lambda$ ) e a amplitude (a), desde uma escala nanométrica até micrométrica.

Métodos: Foram fabricadas amostras de polidimetilsiloxano (PDMS) com topografias nanométricas e micrométricas em gradientes lineares de direção $(\lambda=361 \mathrm{~nm}-10 \mu \mathrm{m} ; \mathrm{a}=16 \mathrm{~nm}-2 \mu \mathrm{m})$. ASCs foram cultivadas nas amostras de PDMS, fabricadas conforme os padrões anteriormente definidos e estimuladas com $1 \mathrm{ng} / \mathrm{mL}$ de TGF- $\beta 1$ para induzir sua diferenciação em SMC. Após 7 dias, a diferenciação foi avaliada utilizando-se marcação para SM22-a em microscópio de imunofluorescência.

Resultados: Adesão, proliferação e alinhamento de ASCs ocorreram preferencialmente nas porções micrométricas do gradiente topográfico. Observou-se, também, que escalas nanométricas inibiram a diferenciação de ASCs em SMC quando estimuladas com TGF- $\beta 1$ (One-way ANOVA, $\mathrm{P}<0,001$ ).

Conclusão: Os presentes achados indicam meios para bloquear ou induzir a diferenciação de ASCs em SMC, respectivamente pela utilização de topografias direcionais nano e micrométricas, bem como as melhores configurações de topografia para a alcançar camadas celulares alinhadas para a fabricação de enxertos vasculares por meio da engenharia de tecidos. 


\section{EXPOSIÇÃO DE PÔSTERES - SBCCV}

\section{PO 66}

\section{Banda anômala de ventrículo direito com passado de PCA e importante repercussão hemodinâmica: relato de caso}

Vitória Mikaelly da Silva Gomes, Gustavo Maffra Monteiro, Alana Francisca Machado Melo, Alanderson da Costa Moreira dos Santos, Marina Mendes Melo, Igor Medeiros Sena, Josinaldo Santos da Costa, Vanessa Késsia Silva Teixeira de Moura, Bruna Gomes de Castro, João Victor Campos da Silva, Christian José David Cabanillas Fernández, José da Silva Leitão Neto, José Kleberth Tenório Filho, Francisco Siosney Almeida Pinto, José Wanderley Neto

Introdução: A banda anômala de ventrículo direito é uma malformação na qual um feixe muscular anômalo cruza o ventrículo direito e o divide em uma parte proximal e outra distal, criando um significativo gradiente de pressão. Segundo CROTI (2008), 80 a $90 \%$ dos casos estão associados à CIV, sendo a estenose valvar pulmonar o segundo achado mais frequente. Apresenta incidência de 0,5 a $2 \%$ e é mais comum no sexo masculino.

Objetivo: Descrever um relato de caso de banda anômala de ventrículo direito em paciente com importante repercussão hemodinâmica.

Métodos: Estudo descritivo, observacional, transversal, de relato de caso de paciente com banda anômala de ventrículo direito.

Resultados: Paciente de 8 anos de idade, sexo feminino, procurou atendimento ambulatorial com queixa de dispneia que piorava aos esforços e estava associada à edema nos MMII. Possuía um passado cirúrgico de correção de PCA e ao exame físico apresentou sopro sistólico em borda esternal esquerda alta. O eletrocardiograma evidenciou sobrecarga de ventrículo direito. O ecocardiograma mostrou o ventrículo direito com hipertrofia importante, banda anômala intraventricular e VSVD com obstrução dinâmica, valva pulmonar espessada e comunicação interventricular ampla, além de insuficiência valvar mitral e tricúspide discretas. A radiografia de tórax evidenciou cardiomegalia à custa de átrio direito e ventrículo direito. A paciente foi submetida a tratamento cirúrgico, com correção dos defeitos simultâneos e desobstrução entre a via de entrada e saída com reconstrução do ventrículo direito, seguindo com boa evolução.

Conclusão: A correção cirúrgica apresenta bom prognóstico e visa evitar hipertrofia ventricular nos assintomáticos e piora hemodinâmica nos sintomáticos.

\section{PO 67}

\section{O prejuízo da função respiratória em pacientes submetidos a cirurgia cardíaca e sua relação com a dor e outros fatores}

Thiago Henrique Silva, Luiz Alberto de Melo, Luís Augusto Franzão Vasconcelos, Vivaldo Logrado Júnior, Ricardo Rodrigues Goulart, Italo Borges Martins

Introdução: A prevalência da dor referente à esternotomia é causa de redução na função pulmonar de pacientes conforme citado por vários autores. É importante entender como esta dor atua e quais outros fatores estão associados para que possamos garantir melhor recuperação pós-operatória.

Objetivo: Este trabalho tem como objetivo pesquisar as fontes que correlacionam a dor e o prejuízo da função respiratória, investigando também como outros fatores ligados à cirurgia influenciam a dor pós-cirurgia e, consequentemente, a respiração do paciente.

Métodos: Revisão bibliográfica, descritiva, qualitativa, que teve como fonte de pesquisa uma filtragem nos sites de busca Scientific Eletronic Library Online (SciELO), MDCONSULT e livros da biblioteca da Universidade Federal do Tocantins (UFT).

Resultados: Giacomazzi mediram o volume inspiratório máximo e a dor subjetivamente em uma escala de 0 a 10, conforme indicação do paciente. Neste caso, foi obtida uma razão com desvio relativamente baixo ( $r=0,277$ e $P=0,04)$. Este estudo confirma a relação específica entre dor e prejuízo da função respiratória. Números mais discrepantes da redução da função pulmonar foram encontrados em outros trabalhos que também apresentaram circulação extracorpórea no procedimento cirúrgico, confirmando uma relação com essa abordagem cirúrgica.

Conclusão: Fica claro que a dor é fator importante no prejuízo da função respiratória, formando a respiração superficial, e que o método e a abordagem cirúrgica influenciam nessa ocorrência. Não foi encontrado nenhum valor que recomendasse a modificação de qualquer abordagem cirúrgica. Nenhum trabalho observou recuperação plena da função respiratória antes do 5 dia pós-operatório. 


\section{PO 68}

\section{Influência dos hábitos tabágicos sobre desfechos operatórios em cirurgia cardiovascular}

Lucas Molinari Veloso da Silveira, Ana Paula Tagliari, Orlando Carlos Belmonte Wender

Introdução: Jones et al. demonstraram existência de maior risco de complicações pulmonares pós-operatórias em pacientes tabagistas comparados aos não tabagistas. Todavia, a maioria dos estudos negligência tabagistas em abstinência, população bastante representativa na cirurgia cardiovascular.

Objetivo: Avaliar a incidência de complicações operatórias imediatas (até a alta hospitalar) de cirurgia cardiovascular, estratificando os pacientes conforme o hábito tabágico.

Métodos: Análise retrospectiva de uma coorte de pacientes submetidos a cirurgia cardiovascular em hospital terciário de Porto Alegre (RS) entre maio de 2015 e agosto de 2016. Os pacientes foram estratificados conforme o hábito tabágico. As análises estatísticas foram realizadas utilizando o software SPSS 22.0, e um valor de $\mathrm{P}<0,05$ foi considerado significativo.

Resultados: Foram incluídos 203 pacientes com média de idade de 61,46 anos e 62,4\% do sexo masculino. Com relação ao hábito tabágico, 16,2\% eram tabagistas ativos, 40,1\% tabagistas em abstinência e 43,7\% não tabagistas. Ao analisarmos os desfechos óbito, infarto agudo do miocárdio e acidente vascular cerebral, não foi constatada diferença estatisticamente significativa entre os grupos. Em relação às complicações pulmonares no pós-operatório, foram observadas pequenas diferenças na incidência de broncopneumonia, incidência de insuficiência respiratória aguda e tempo de ventilação mecânica. Os pacientes não tabagistas apresentaram melhores resultados, porém, sem significância estatística.

Conclusão: Estes resultados não demonstraram diferença significativa em desfechos pós-operatórios a depender do hábito tabágico dos pacientes. Acreditamos que a continuidade e o aumento do tamanho da amostra deste estudo auxiliarão a responder mais robustamente sobre a influência do tabagismo, mesmo quando já cessado, nos desfechos pós-operatórios.

\section{PO 69}

\section{Internamentos por cardiopatia congênita no estado de alagoas entre janeiro de 2010 e dezembro de 2015}

João Victor Campos da Silva, José Wanderley Neto, Francisco Siosney Almeida Pinto, Christian José David Cabanillas Fernández, José da Silva Leitão Neto, José Kleberth Tenório Filho, Marina Mendes Melo, Bruna Gomes de Castro, Alana Francisca Machado Melo, Alexia Carneiro de Almeida, Igor Medeiros Sena, Leandro Castelo Alves, Tullazy Cavalcante Torres, Mariana Ramos Andion, Klaus Anton Tyrrasch

Introdução: As cardiopatias congênitas são as malformações graves mais frequentes, com alta mortalidade no $1^{\circ}$ ano de vida. Possuem incidência entre 4 e 19/1.000 nascidos vivos. No Brasil, em 2008, corresponderam a aproximadamente 19\% da mortalidade em menores de um ano, perfazendo a segunda principal causa de óbito nessa faixa etária.

Objetivo: Caracterizar os internamentos por cardiopatia congênita no estado de Alagoas durante o período de janeiro de 2010 a dezembro de 2015.

Métodos: Trata-se de um estudo ecológico, realizado a partir de dados obtidos do sistema TabNET do Datasus. As variáveis estudadas foram sexo, idade, permanência, custo e taxa de mortalidade.

Resultados: Durante o período estudado houve um total de 94.687 internações por cardiopatia congênita no Brasil, sendo 1.039 no estado de Alagoas, com taxa de mortalidade de 7,99\%, representando um custo total de $\mathrm{R} \$ 8.794 .183,80$. O sexo feminino foi responsável por 548 (52,74\%) das internações, com taxa de mortalidade de 6,57\%, sendo a faixa etária mais acometida os menores de um ano (15,32\%). A taxa de mortalidade no sexo masculino foi de 9,57\%, com faixa etária mais acometida sendo os menores de um ano (16,70\%). Entre os anos de 2013 e 2014 observou-se uma queda de 19,23\% no número de internamentos, mantendo-se essa taxa de decrescimento em comparação com o ano de 2015.

Conclusão: Ainda que haja um decréscimo no número de internações, é necessário que se tenha uma maior abrangência do serviço de pré-natal e do rastreio precoce dessas doenças, ambos concorrendo para prevenção e diagnóstico precoces. 


\section{EXPOSIÇÃO DE PÔSTERES - SBCCV}

\section{PO 70}

\section{Fístula aorta-átrio direito: relato de um caso raro}

Henrique Madureira da Rocha Coutinho, Joaquim Henrique de Souza Aguiar Coutinho, João Carlos Jazbik, Ana Carolina Veltri Pacheco, Gustavo Kikuta, Bernardo Ferreira Americano do Brasil, Pedro Verbicário dos Santos Leite

Introdução: Fístulas entre a aorta e o átrio direito são uma rara condição clínica, normalmente oriundas de processo infeccioso ou traumático (procedimento cirúrgico, dissecção de aorta, entre outros), além de malformação congênita. As fístulas aortocamerais são malformações vasculares extremamente raras, sendo sua apresentação clínica, história natural e opções de tratamento ainda pouco conhecidos. Essas comunicações podem ser originárias de qualquer seio de Valsalva, sendo extremamente raro quando surge do seio não coronariano, como no caso a ser descrito.

Objetivo: Relatar uma doença pouco comum na literatura, mas de importante relevância clínica.

Métodos: Foi estudado o caso de um paciente de 40 anos, sexo masculino, natural do Ceará, estivador, que apresenta há 1 ano quadro de dispneia progressiva, edema de membros inferiores, turgência jugular patológica, hepatomegalia, ausculta de sopro diastólico mais evidente em região paraesternal direita. Ecocardiograma apresentou comunicação entre aorta (seio não coronariano) e o átrio direito com shunt importante E-D, aumento das quatro câmaras, disfunção de grave de VD e disfunção moderada de VE. Valvas cardíacas sem alterações.

Resultados: Paciente submetido à correção cirúrgica com resolução completa do shunt intercavitário, confirmado por ecocardiograma e melhora expressiva de sua condição clínica, recebendo alta hospitalar em 72 horas.

Conclusão: Fístulas entre a aorta e o átrio direito são raras e de difícil diagnóstico e não há uma definição na literatura atual quanto ao melhor tratamento a ser realizado. É de extrema importância o conhecimento desta condição, pois a suspeição clínica é fundamental em seu diagnóstico.

\section{PO 71}

\section{Implante de prótese metálica aórtica invertida na estrutura da prótese mitral biológica degenerada por meio de técnica minimamente invasiva}

Jeronimo Antonio Fortunato Junior, Wilson Nkundumukiza, Jeferson R. Sesca

Introdução: A retirada cirúrgica de prótese biológica mitral degenerada está associada a várias complicações perioperatorias graves, incluindo disjunção atrioventricular, ruptura de parede livre de ventrículo esquerdo, lesão de artéria circunflexa e escape paravalvar tardio. Alexander Geha, em 1996, descreveu uma técnica de implante de valva aórtica invertida sobre a estrutura da prótese biológica após retirada somente de seus folhetos sem explante completo.

Objetivo: Relatar um caso em que foi utilizada a técnica descrita por Alexandre Geha e demonstrar seu resultado.

Métodos: Paciente do sexo masculino, de 50 anos, com prótese biológica mitral implantada 15 anos antes, com degeneração grave e necessidade de retroca valvar. Foi realizada cirurgia cardíaca minimamente invasiva através de incisão transmamilar de $5 \mathrm{~cm}$ em $4^{\circ}$ espaço intercostal direito, com canulação femoral e fibrilação hipotérmica sem clampeamento aórtico. Não foram realizadas dissecções das estruturas cardíacas e o acesso ao átrio esquerdo foi por via transpericárdica.

Resultados: Foi implantada, com sucesso, uma prótese aórtica número 25 (St. Jude), de maneira invertida sobre o anel da estrutura da prótese biológica remanescente após excisão de seus folhetos.O tempo de circulação extracorpórea foi de 130 minutos. 0 sangramento pos operatório foi de $300 \mathrm{~mL}$, sem necessidade de reposição de hemoderivados. O paciente permaneceu 2 dias na UTI e 3 dias no hospital. É acompanhado há 1 ano sem intercorrências; o ecocardiograma demostrou prótese adequadamente posicionada sem refluxo paravalvar, classe funcional I (NYHA) e regime de anticoagulação oral.

Conclusão: Neste relato, pudemos confirmar que a técnica descrita é factível e pode também ser usada com cirurgia cardíaca minimamente invasiva, reduzindo mais ainda as dissecções cirúrgicas necessárias na esternotomia mediana e as comorbidades relacionadas. 


\section{PO 72}

\section{Correction of coarctation and ascending aortic aneurysm by extra-anatomical shunt combined with atrioseptoplasty: case report}

Mariana Pimentel Pastor, Marcio Pimentel Fernandes, Marcelo José Ferreira Soares, Cristiane de Carvalho Singulane, Diego Novelli

Introduction: Aortic coarctation (AoCo) usually is corrected in childhood; however, many patients reach adulthood without diagnosis. The correction in adulthood can be difficult because it is associated with comorbidities such as atrial septal defects (ASD) and the presence of bicuspid valve.

Objective: Report a rare case in our hospital.

Methods: Analysis and data collection by computerized patient records review.

Results: Case report: A 42-year-old woman with history of hypertension sought treatment and underwent tests where identified aortic aneurysm, AoCo and ASD, being electively underwent corrective heart surgery.

Discusion: Congenital AoCo is a malformation that can lead to premature death, so the correction is made in the first years of life of the patients. When diagnosis is late, it is not always possible to perform the resection of the coarctation area, due to the anatomical local conditions, the caliber side and difficulty in mobilization. The disclosure of this disease is the correction needs occur early.

Conclusion: Surgical treatment of AoCo in adults with extracorporeal circulatory support, aneurysm repair and ASD closure presents itself as an effective option in cases requiring greater aortic clamping.

\section{PO 73}

\section{Avaliação da analgesia realizada em pós-operatório de cirurgia cardíaca}

Bernardo Ferreira Americano do Brasil, Joaquim Henrique de S. A. Coutinho, Gustavo Kikuta, Henrique Madureira da Rocha Coutinho, Pedro Verbicário, Joao Carlos Jazbik, Ana Carolina Veltri Pacheco, Carla Coutinho

Introdução: O quadro de dor é um assunto frequentemente discutido no pós-operatório de cirurgia cardíaca. Vimos demonstrar o quão simples pode ser feito o controle do quadro álgico e desmitificar que a cirurgia cardíaca acarreta um quadro de sofrimento exacerbado ao paciente operado.

Objetivo: Elucidar os níveis de dor em pós-operatório de cirurgia cardíaca, comparando diferentes tipos de analgesia do $1^{\circ}$ ao $3^{\circ}$ dia de pós-operatório. Comparar diversificadas vias de acesso por videocirurgia assistida ou toracotomias tradicionais e analisar o que tais abordagens refletem nos pacientes.

Métodos: Estudo prospectivo, comparativo, com 75 pacientes submetidos a cirurgia cardíaca em um hospital universitário, no qual nos baseamos em uma escala de dor numérica e visual, dividindo em diferentes níveis de dor pós-operatória denominados grupos A (1-3), B (4-6) e C (6-10).

Resultados: Vimos que o maior percentual de pacientes (76\%) se encontra em nível A de dor apenas com analgesia branda. Não obstante, os pacientes submetidos à videocirurgia assistida em um primeiro momento apresentaram níveis álgicos maiores, nível A de dor (50\%), porém, nos dias subsequentes, mostram redução mais abrupta na analgesia comparando as toracotomias tradicionais.

Conclusão: Diante de nosso trabalho, conseguimos desmitificar que a cirurgia cardíaca propicia um pós-operatório com maior sofrimento ao paciente e difícil manuseio. Verificamos que é factível manter os pacientes apenas com analgésicos não opioides o pós-operatório imediato. O opiode se apresentou como opção terapêutica em 48\% dos pacientes em $1^{\circ}$ dia de pós-operatório e 15\% no $2^{\circ}$ dia. 


\title{
EXPOSIÇÃO DE PÔSTERES - SBCCV
}

\section{PO 74}

\section{Dissecção espontânea de artéria coronária: revisão de dois casos em centros de referência}

\author{
Vitor Prochnow, Vinícius Felipe Czapla, Emma Salazar, Rogério Abrahão, Álvaro Schmidt Albrecht
}

\begin{abstract}
Introdução: Dissecção espontânea de artéria coronária representa uma causa infrequente de síndrome coronariana aguda, com
\end{abstract} altos índices de mortalidade e terapêutica ideal ainda não estabelecida.

Objetivo: Apresentar dois casos manejados cirurgicamente com sucesso.

Métodos: Caso 1: E.M.M., sexo masculino, 48 anos, previamente hígido, sem antecedentes familiares de doença cardiovascular. Iniciou quadro de tontura e dor retroesternal após esforço. ECG e enzimas cardíacas diagnosticaram infarto agudo do miocárdio (IAM). Cateterismo e ecocardiograma transtorácico (ETT) mostraram dissecção do segmento proximal e médio da artéria descendente anterior (DA). Realizou-se cirurgia de revascularização miocárdica (CRM) com interposição da artéria mamária para DA e safena para a artéria diagonal; sem intercorrências. Caso 2: M.B.K., sexo feminino, 54 anos, previamente hígida. História familiar de IAM. Apresentou dor súbita retroesternal. ECG: IAM em parede inferior, mais acometimento de ventrículo direito. Cineangiocoronariografia: dissecção coronariana direita em todo seu segmento. Inicialmente, foi eleito tratamento conservador. Após 5 dias, desenvolveu derrame pericárdico com suspeita de ruptura ventricular. Foi submetida à cirurgia de urgência. Realizouse correção ventricular com cobertura extensa de pericárdio bovino em sutura contínua de prolene 4-0 e pontos separados para reforço. Não houve CRM.

Resultados: Caso 1: Cineangiocoronariografia demonstrou aspecto favorável, com paciente estável, sem recorrência de angina ou eventos. Caso 2: Paciente se manteve estável durante o pós-operatório imediato e tardio, sem recorrência de eventos.

Conclusão: A CRM representa o tratamento de escolha na presença de instabilidade hemodinâmica, dissecção de múltiplos vasos, tronco coronariano esquerdo e falha da terapia conservadora. Tem demonstrado ótimos resultados a curto e longo prazos.

\section{PO 75}

\section{Treatment of a pedunculated pseudoaneurysm in the ascending aorta with penetrating aortic ulcer associated: case report}

Mariana Pimentel Pastor, Marcio Pimentel Fernandes, Marcelo Jose Ferreira Soares, Cristiane de Carvalho Singulane, Diego Novelli

Introduction: The presence of pseudoaneurysm associated with penetrating aortic ulcer in the ascending portion is a rare case and poorly reported. It is interesting the disclosure of cases of patients suffering from these injuries. The echocardiography and angiotomography evaluations enable the correct early diagnosis and thus treatment in a timely manner. This study reports the treatment of a pedunculated pseudoaneurysm with penetrating aortic ulcer (PAU) associated with the ascending aorta in a 60 years old patient with chest pain and systemic arterial hypertension.

Objective: Report a rare case in our hospital.

Methods: Analysis and data collection by computerized patient records review.

Results: Case report: In the diagnostic investigation, pedunculated pseudoaneurysm in the ascending aorta with penetrating aortic ulcer associated was found, treated by surgery via median sternotomy with cardiopulmonary bypass. The procedure was performed successfully and the patient progressed steadily after surgery, being in outpatient treatment, asymptomatic.

Discussion: The penetrating ulcer in the ascending aorta should be treated as aortic dissection type A due to the large desiccant potential that it presents. Thus, accurate diagnosis and early intervention minimizes the risks and potential complications related to morbidity and mortality of this disease.

Conclusion: The aortic diseases are responsible for high cardiovascular morbidity and mortality rates and it remain a challenge for clinical cardiologists, interventionists and cardiovascular surgeons. PAU with saccular pseudoaneurysm treatment and its complications must respect the same train of thought, deserving thus recognition, fast diagnosis and suitable treatment ensuring expectancy and quality of life for the patient. 


\section{PO 76}

\section{Ducto arterioso persistente: relato de caso}

Igor Leão Gomes Leahy, lago Moura Aguiar, Cicero Felipe Paes de Araujo Costa, Alfredo Aurélio Marinho Rosa Filho, Júlio César Claudino dos Santos, Lyles Regina Machado Falcão, Maria Eduarda Prudente Kunzler Alves

Introdução: $O$ ducto arterioso é um vaso que comunica a artéria pulmonar com a aorta no feto. O fechamento funcional desse ducto no recém-nascido a termo ocorre com 12 a 15 horas de vida, e o permanente, com 5 a 7 dias, alcançando, em alguns casos, até o $21^{\circ}$ dia. Porém, pode persistir no decorrer da vida. A persistência sintomática é definida pela presença do sopro cardíaco, pela taquicardia, pelo precórdio hiperdinâmico e pelo aumento da amplitude de pulso.

Objetivo: O objetivo deste estudo é relatar, após a injeção de solução salina, a persistência do ducto arterioso em um coração cadavérico humano e sua correlação com a clínica do paciente a fim de utilizá-lo como diagnóstico diferencial para outras modalidades de insuficiência cardíaca congestiva.

Métodos: Trata-se de um estudo com injeção de solução salina no terço médio da aorta ascendente por intermédio de uma sonda de poliuretano suturada à aorta com fio de algodão conectada a uma seringa de $50 \mathrm{~mL}$.

Resultados: Após análise, verificou-se que, quando se fazia a injeção de solução salina, esta desembocava no terço cranial do tronco pulmonar, confirmando a comunicação. Os indivíduos que apresentam a forma clássica da doença (shunt esquerda-direita) manifestarão sinais clínicos de insuficiência cardíaca congestiva esquerda e os indivíduos que possuem shunt funcional direitaesquerda apresentarão hipertrofia ventricular direita e policitemia.

Conclusão: O diagnóstico definitivo é obtido por ultrassom e angiografia. As terapias utilizadas para oclusão do ducto incluem a ligadura cirúrgica por toracotomia e a oclusão percutânea via artéria ou veia femoral.

\section{PO 77}

\section{Comunicação interventricular após revascularização do miocárdio}

\section{Andrea Cristina Oliveira Freitas, Wladmir Faustino Saporito, Nicolle Martin, Adilson Casemiro Pires}

Introdução: Comunicação interventricular (CIV) é uma complicação mecânica do infarto agudo do miocárdio (IAM). Ocorre pelo cisalhamento entre área necrosada e viável, causando uma falha no septo interventricular. A reperfusão precoce teve impacto significativo na redução da incidência da CIV nos últimos anos.

Objetivo: Relatar a ocorrência de CIV após reperfusão cirúrgica, complicação rara da revascularização do miocárdio.

Métodos: Paciente do sexo feminino, 58 anos, hipertensa, procurou a emergência em IAM inferior Killip I. Optou-se pelo tratamento clínico exclusivo. Após 8 dias, evoluiu com angina pós-infarto, sendo conduzida para tratamento cirúrgico de urgência.

Resultados: A paciente foi submetida à revascularização do miocárdio com circulação extracorpórea. Evoluiu sem intercorrências até o terceiro dia, quando apresentou parada cardíaca e foi submetida à reanimação por 32 minutos. Retornou em ritmo sinusal, porém hemodinamicamente instável, e surgimento de sopro sisto-diastólico em toda a borda esternal esquerda. Ecocardiograma à beira do leito identificou ruptura do septo interventricular, ausente em exame pré-operatório. Por apresentar sinais clínicos de encefalopatia anóxica grave, e após constatação de lesão neurológica irreversível, a cirurgia de urgência foi contraindicada. A paciente evoluiu a óbito dois dias depois.

Discussão: A CIV é uma complicação mecânica grave do IAM, que deve ser suspeitada na presença de sopro cardíaco novo. Ocorre entre $06^{\circ}$ e o $10^{\circ}$ dia pós-infarto e acomete de 1 a 3\% dos pacientes. Pode ocorrer após trombólise química ou, raramente, após angioplastia.

Conclusão: Na literatura consultada, não há nenhum outro relato de ocorrência de CIV em pós-operatório recente de revascularização do miocárdio. 


\section{EXPOSIÇÃO DE PÔSTERES - SBCCV}

\section{PO 78}

\section{O impacto das novas tecnologias diagnósticas no tratamento das doenças cardiovasculares - relato de caso}

Nelson Americo Hossne Junior, Eduardo Gregorio Chamlian, Renato Azevedo Junior, Roberto Caldeira Cury, Mauricio Rodrigues Jordão, Jairo Alves Pinheiro Junior, Fernando Henrique Carlos de Souza, Jussara Bianchi Castelli, Walter José Gomes

Introdução: A sarcoidose é uma doença crônica, sem etiologia definida, caracterizada pela presença de granulomas não caseosos que podem afetar vários órgãos ou tecidos. Em pacientes com comprometimento cardíaco, os granulomas sarcoides geralmente são encontrados no miocárdio ou no endocárdio. A doença geralmente se apresenta como uma síndrome de insuficiência cardíaca com distúrbios de condução, associada ou não a arritmias ventriculares. A pericardite constritiva é raramente descrita em pacientes com sarcoidose.

Objetivo: Relatamos um caso de uma mulher de 50 anos com queixa de dispneia progressiva há cerca de 6 meses, associada a dor torácica de causa desconhecida.

Métodos: Relato de caso.

Resultados: A ressonância nuclear magnética de coração com uso de gadolínio mostrou realce tardio envolvendo todo o pericárdio, difusamente mais acentuado em região apical biventricular, compatível com pericardite constritiva. O ecocardiograma transtorácico revelou um pericárdio espessado com sinais de fisiologia constritiva. A paciente foi submetida a uma ressecção anterior extensa, interfrênica, do pericárdio, com melhora significativa dos sintomas. A análise histopatológica do pericárdio confirmou uma pericardite fibrinosa com a presença de alguns granulomas de padrão sarcoide. Após 6 dias a paciente recebeu alta hospitalar em boas condições clínicas, em uso de betabloqueador, inibidor da enzima de conversão da angiotensina, associado a prednisona, 40 mg ao dia.

Discussão: Em revisão de literatura encontramos apenas 3 relatos de caso da associação sarcoidose e pericardite constritiva.

Conclusão: $O$ auxílio de exames avançados de imagem foi fundamental para o diagnóstico precoce, assim como a realização do tratamento adequado.

\section{PO 79}

\section{Caracterização das extubações acidentais ocorridas em pacientes em pós-operatório imediato na unidade coronariana do Hospital Calixto Midlej Filho, Itabuna, Bahia}

Larissa Cavalcante Nunes, Milena Vasconcellos Silva, Décio Cardoso da Silva Junior, Luiz Henrique Gualberto Junior, Kitiana Carvalho Pacheco, Laila Vasconcelos Galvão, Camila de Morais Masini, Amilton Santos Silva, Stefanie Santos Marinho Bonina, Denis Albuquerque Silva Dias, Juliana Souza Lago

Introdução: As extubações acidentais no pós-operatório de cirurgia cardiovascular caracterizam um evento adverso do cuidado e comprometem o estado clínico do paciente, ao retardar a recuperação e elevar os riscos de hipoxemia, atelectasia, pneumonia associada à ventilação mecânica (PAV) e instalibilidade hemodinâmica; além de representar maior custo hospitalar e tempo de ventilação mecânica, quando associadas a quadros de reintubação.

Objetivo: Caracterizar e quantificar as extubações acidentais ocorridas na unidade coronariana (UCO), em 2015, entre os pacientes em pós-operatório imediato de cirurgia cardíaca.

Métodos:Trata-se de um estudo retrospectivo, realizado pela análise dos impressos de notificação de eventos adversos, preenchidos pelos enfermeiros da UCO, no ano de 2015. Os dados foram armazenados em um banco de dados no software SPSS versão 19 , no qual foram realizados os cálculos de estatística descritiva.

Resultados: Foram identificadas 5 notificações de extubação acidental, todas ocorridas em indivíduos do sexo masculino. Entre as causas registradas, 2 (40\%) eventos ocorreram durante o manuseio do paciente pela equipe multidisciplinar, como banho e realização de radiografia de tórax; 1 (20\%) caso se deu pela baixa pressão no cuff da cânula endotraqueal, com migração acidental do tubo para a boca e os 2 (40\%) últimos registros foram consequência da tração acidental pelo paciente por agitação psicomotora. Somente dois pacientes necessitaram de reintubação; os demais (60\%) já apresentavam critérios de extubação assistida sem indicação de assistência ventilatória.

Conclusão: As notificações de eventos adversos facilitam a identificação dos indicadores da qualidade da assistência prestada e devem ser vistos como meios de melhora e qualificação da equipe multidisciplinar. 


\section{PO 80}

\section{Revascularização do miocárdio com enxerto bilateral de artérias torácicas internas via minitoracotomia esquerda sem o auxílio da circulação extracorpórea. Primeiro relato de caso realizado no Brasil}

Ricardo Barros Corso, Isaac Azevedo Silva, Helmgton José Brito de Souza, Glauco Kalil da Silva Pina, Marcus Vinicius Nascimento dos Santos

Introdução: A revascularização do miocárdio (RM) via minitoracotomia esquerda (MTE) tem sido preconizada por alguns centros com o objetivo de reduzir o trauma cirúrgico. O uso rotineiro de duplo enxerto de ATI dissecado sob visão direta na RM via MTE foi mais recentemente descrito.

Objetivo: Descrever um primeiro caso operado em nosso serviço de RM SEM CEC via MTE com o uso de duplo enxerto de ATI.

Métodos: Paciente do sexo feminino, 36 anos, diabética tipo I. Investigação pré-operatória para cirurgia ortopédica revelou isquemia moderada. Cateterismo cardíaco revelou oclusão de circunflexa de pequena importância, lesão proximal de 80\% em descendente anterior (DA) e de $90 \%$ em origem de grande ramo diagonal (DGI). Em outubro de 2016 realizou-se RM via MTE. Utilizou-se o afastador ThoraTrack ${ }^{\circledR}$. Outro afastador foi posicionado em região subxifóidea. Fez-se a liberação do mediastino anterior e abertura da pleura direita. Para a dissecção das ATIs utilizou-se dissector em gancho (Ultracision). Utilizou-se um segmento de $10 \mathrm{~cm}$ da ATID para a confecção de enxerto composto em Y artificial. Fez-se anastomose distal da ATIE para a a. DA e da ATID para a a. DGI com o auxílio do estabilizador cardíaco (Octopus ${ }^{\oplus}$ ) e de shunt intracoronariano (Clearview $\left.{ }^{\circledast}\right)$.

Resultados: Como complicação pós-operatória houve piora transitória da disfunção renal e necessidade de redrenagem percutânea de derrame pleural.

Conclusão: A RM via MTE SEM CEC com o uso de ambas as ATIs foi reprodutível em nosso serviço e pode ser uma alternativa em casos selecionados. Este é o primeiro caso no Brasil operado com essa técnica de nosso conhecimento.

\section{PO 81}

\section{Dados epidemiológicos dos pacientes com dupla disfunção valvar submetidos à correção cirúrgica na Santa Casa de Misericórdia de Maceió}

Kamilla Peixoto Bandeira, Vera Nascimento Gomes Victoria, Karine Nascimento Chaves, Tullazy Cavalcante Torres, Vitória Mikaelly da Silva Gomes, Alana Francisca Machado Melo, Bruna Gomes de Castro, Marina Mendes Melo, João Victor Campos da Silva, Alexia Carneiro de Almeida, Bianca Brenda Xavier Costa, Alanderson da Costa Moreira dos Santos, Peter Conde Vidal Júnior, Rafaela da Hora Sales, Laio Cajú Wanderley, Francisco Siosney Almeida Pinto, José Wanderley Neto

Introdução: A dupla lesão valvar corresponde à existência de estenose associada à insuficiência em uma mesma valva. Geralmente há predomínio de uma das lesões, mas a gravidade de ambas pode ser semelhante, o que é chamado de dupla lesão balanceada.

Objetivo: Avaliar aspectos clínicos e epidemiológicos dos pacientes com dupla lesão valvar submetidos à correção cirúrgica.

Métodos: Foram avaliados, retrospectivamente, 121 pacientes portadores de dupla lesão valvar, tratados cirurgicamente no Serviço de Cirurgia Cardiovascular da Santa Casa de Misericórdia de Maceió entre janeiro de 2013 e setembro de 2016. Foram analisadas as variáveis sexo, idade, Euroscore e correção cirúrgica.

Resultados: Dos 121 pacientes submetidos à correção cirúrgica de dupla lesão valvar, 66 (54,5\%) eram do sexo feminino e 55 $(45,5 \%)$ do sexo masculino. Os pacientes possuíam entre 17 e 86 anos de idade, e o Euroscore variou de 0,63\% a 12,5\%. Nos pacientes que possuíam apenas dupla lesão mitral (DLM), 5 (8,9\%) apresentaram predomínio de estenose mitral, 0 (0\%) predomínio de insuficiência mitral e 51 (91,1\%) DLM balanceada, totalizando 56 pacientes (46,3\%). Dos pacientes que apresentaram apenas dupla lesão aórtica (DLAo), 13 (26,5\%) possuíam predomínio de estenose aórtica, 2 (4,1\%) predomínio de insuficiência aórtica e 34 $(69,4 \%)$ DLAo balanceada, totalizando 49 pacientes (40,5\%). Dezesseis pacientes (13,2\%) apresentaram simultaneamente DLM e DLAo. Como tratamento cirúrgico, todos os pacientes receberam implante valvar.

Conclusão: Verificou-se que a DLM é a mais frequente e que ocorre predominância das duplas lesões valvares no sexo feminino. $O$ implante valvar foi o único tratamento cirúrgico realizado. 


\title{
EXPOSIÇÃO DE PÔSTERES - SBCCV
}

\section{PO 82}

\section{Transfixação cardíaca por projétil de arma de pressão}

\author{
Guilherme Krahl, Vinícius Felipe Czapla
}

Introdução: Ferimentos por arma de fogo com acometimento torácico são traumas comuns; entretanto, ferimentos por acidentes com armas de pressão raramente encontram-se associados a lesões complexas.

Objetivo: Relatar um caso e manejo cirúrgico de um trauma cardíaco por arma de pressão.

Métodos: G.V.V., 14 anos, sexo masculino, foi atingido por disparo de uma espingarda de pressão em hemitórax esquerdo, sem orifício de saída. Atendido na cidade de origem, recebeu suporte hemodinâmico com solução cristaloide. À tomografia computadorizada de tórax, evidenciou-se grande derrame pericárdico e projétil alojado no mediastino médio. Paciente foi encaminhado para o Hospital São Vicente de Paulo de Passo Fundo para tratamento cirúrgico, apresentando taquicardia, sonolência, turgência jugular e abafamento de bulhas. Realizou-se esternotomia mediana e pericardiotomia que revelou grande quantidade de sangue e coágulos. Identificado o orifício de entrada na parede anterior do ventrículo direito, foi realizada sutura com polipropileno 4.0 ancorada em pericárdio. Sob circulação extracorpórea e atriotomia esquerda foi retirado o projétil alojado junto à parede posterior. A atriotomia direita, por sua vez, revelou perfuração do folheto anterior da tricúspide que foi corrigida por sutura simples. Não houve observância do pertuito de passagem do projétil entre as câmaras.

Resultados: O paciente apresentou evolução satisfatória, recebendo alta hospitalar no 70 dia, sem sequelas. O ecocardiograma pós-operatório não evidenciou alterações anatômicas residuais.

Conclusão: Quando atendido em um centro terciário, a cirurgia permite retirada do projétil, identificação de danos nas estruturas intracardíacas e diminuição da necessidade de tratamento complementar em um segundo instante, reduzindo morbidade e mortalidade.

\section{PO 83}

\section{Comparativo do perfil dos pacientes submetidos a cirurgia cardíaca no primeiro (2013) e no terceiro (2015) ano de uma unidade coronariana no sul da Bahia}

Larissa Cavalcante Nunes, Aritana Pereira Ramos, Décio Cardoso da Silva Junior, Kitiana Carvalho Pacheco, Milena Cristina Vasconcellos Silva, Rafael Vinícius Santos Cruz, Solon Ramos Santos Cruz, Jan Reinel De Castro

Introdução: Segundo a OMS, as doenças cardiovasculares são responsáveis por mais da metade dos óbitos mundiais (WHO, 2005). As terapêuticas cirurgias cardiovasculares proporcionam melhora na sobrevida e na qualidade de vida do indivíduo.

Objetivo: O estudo avaliou o perfil dos pacientes submetidos à cirurgia de revascularização do miocárdio e troca valvar, realizados no HCMFo, nos anos de 2013 e 2015, internados na unidade coronariana.

Métodos: Trata-se de um estudo retrospectivo, realizado pela análise dos registros dos pacientes submetidos às cirurgias cardíacas. Os dados foram avaliados por cálculos de estatística descritiva, no software SPSS versão 19.

Resultados: Considerando a revascularização do miocárdio, em 2013 ocorreram 131 procedimentos, comparado a 107 em 2015, uma redução de 8,16\%. Em ambas as condições predominaram sexo masculino, com 66,4\% em 2013 e 70,09\% em 2015, assim como os internamentos no SUS, com 80,9\% e 78,50\%, respectivamente; a mortalidade reduziu de 3,8\% para 2,8\% entre os anos analisados. Quanto à troca valvar, no primeiro ano foram feitos 61 procedimentos e 49 no terceiro ano, uma redução de $19,67 \%$. Mas, em 2013, houve predomínio do sexo feminino (59\%), enquanto em 2015 mais homens foram operados (53,06\%); manteve-se predomínio de internamentos no SUS, 95,1\% em 2013 e 91,18\% em 2015. A taxa de óbito nesta cirurgia reduziu-se de 1,6\% em 2013 para 0\% em 2015.

Conclusão: Observou-se redução na mortalidade quando comparado o primeiro e o terceiro ano de cirurgias, com predominância de internamentos via SUS, e maior número de operados do sexo masculino, excetuando-se as trocas valvares realizadas em 2013. 


\section{PO 84}

\section{Cirurgia de revascularização miocárdica em paciente com hipercolesterolemia familiar: relato de caso e revisão da literatura}

Thiago Vila Nova, Caio Cesar Cardoso, Ademir Massarico Braz, Celso Reis Ávila, Fabiano André Pereira, Tatiane Lamarca Dias, Eduardo Gregório Chamlian, Nelson Americo Hossne Junior, Walter José Gomes

Introdução: A hipercolesterolemia familiar (HF) é uma doença genética do metabolismo das lipoproteínas que apresenta risco aumentado de doença arterial coronariana (DAC) prematura e reduz a expectativa de vida em seus portadores. Caracteristicamente, pacientes com HF na forma homozigótica apresentam DAC com grave comprometimento das artérias coronárias e intensa calcificação da aorta ascendente, em razão do aumento plasmático de marcadores inflamatórios relacionados à aterosclerose. Com a expansão do diagnóstico dos casos de HF, um maior número de pacientes será avaliado para terapia de revascularização miocárdica (RM).

Objetivo: Relatar condução e escolha de tratamento de revascularização miocárdica em paciente com HF e DAC grave sintomática.

Métodos: Paciente do sexo feminino, 42 anos, com acometimento aterosclerótico grave de todas as artérias coronárias e disfunção ventricular esquerda, considerada para RM, cirúrgica ou percutânea. A opção cirúrgica foi escolhida pelas características da HF, com múltiplas placas instáveis e atividade inflamatória aumentada, e o envolvimento anatômico avançado das artérias coronárias e aorta ascendente.

Resultados: A paciente foi submetida à cirurgia de RM sem circulação extracorpórea e sem manipulação da aorta (técnica anaórtica), com emprego de enxertos arteriais (artérias torácicas internas). Apresentou evolução pós-operatória satisfatória, recebendo alta hospitalar no $4^{\circ}$ dia pós-operatório.

Conclusão: Em pacientes com HF e DAC severa sintomática, o tratamento de RM cirúrgica pode representar a melhor opção pelas características descritas da doença. A técnica anaórtica com enxertos arteriais deve ser fortemente considerada em virtude da redução do risco de dano neurológico e da melhor patência dos enxertos em longo-prazo.

\section{PO 85}

\section{Quando operar aneurisma do seio de Valsalva da valva aortica}

Leonardo Peixoto Lopes Vilar, Francisco Gregori Júnior, Alexandre Noburo Murakami, Rogerio Toshio Teruya, Guilherme Andrade Krawczun, João Ricardo Buono

Introdução: Operar um ASV, seus riscos e benefícios, com exames mais precisos e diagnóstico precoce das moléstias cardíacas.

Objetivo: O ASV é uma doença rara. ASV rotos sintomáticos; com dispneia, edema de MMII com ICC; ECO, CATE, TC, RM, sendo um aliado tanto no diagnóstico precoce da doença, quanto na prevenção de complicações e permite um planejamento cirúrgico mais preciso.

Métodos: R.D.S.F., 45 anos, sexo feminino, branca, brasileira; ASV cúspide não coronariana vaórtica + CIA do Tipo os cirurgia: fechamento do óstio do aneurisma junto ao seio de Valsalva com patch de pericardio bovino+CIA+ressecção do prolapso aneurismático.

Resultados: Sintomas: defeitos cardíacos associados, como dispneia ou ICC, defeito anatômico $>70 \mathrm{~mm}$ e risco de complicação iminente, como rotura, congênita pela CIA. O tratamento cirúrgico precoce do ASV é a escolha em pacientes sintomáticos e em caso de ruptura do aneurisma. O risco operatório é baixo e o resultado a longo prazo é bom. TVAo quando há insuficiência aórtica. RM também pode ser usadas em casos de ASV associados à compressão coronariana.

Conclusão: O AVS é uma doença rara, com CIA, no caso, não coronariana de diâmetro grande. Realizou-se do diagnóstico precoce, e pelo risco de rotura, foi optado pela imediata intervenção cirúrgica, com técnica mais comum de fechamento com placa de pericárdio bovino, correção de ClA associada e ressecção completa do pedículo, para evitar recidivas. Com os diversos recursos técnicos atuais, esses são os melhores caminhos para atingir maior taxa de sobrevivência e melhor qualidade de vida para os pacientes, neste caso com boa recuperação pós-operatória e boa evolução clínica, com seis anos de seguimento, mantendo fração de ejeção normal. 


\section{EXPOSIÇÃO DE PÔSTERES - SBCCV}

\section{PO 86}

\section{Sindrome de Takotsubo em pós-operatório imediato de cirurgia cardíaca}

Pedro Verbicário dos Santos Leite, João Carlos Jazbik, Joaquim Henrique de Souza Aguiar Coutinho, Ana Carolina Veltri Pacheco, Henrique Madureira da Rocha Coutinho, Gustavo Kikuta, Bernardo Ferreira Americano do Brasil, Carla Coutinho, Matheus Brum

Introdução: A cardiomiopatia de Takotsubo caracteriza-se por disfunção ventricular apical aguda e reversível. É uma forma de atordoamento miocárdico com mecanismos diferentes dos observados durante episódios transitórios de isquemia das doenças coronarianas. Ocorre predominantemente em mulheres no período pós-menopausa após exposição a estresse repentino.

Objetivo: O objetivo deste estudo é demonstrar um acometimento raro desta síndrome em uma paciente jovem após cirurgia cardíaca, podendo ser um diagnóstico diferencial em casos equivalentes.

Métodos: Paciente de 26 anos, com quadro de dispneia há 6 meses com piora progressiva. Teste ergométrico normal, ecocardiograma com função ventricular preservada e valva mitral apresentando calcificação reumática, com refluxo severo, sem hipertensão pulmonar. Submetida à troca valvar mitral no dia 13/10. Utilizada cardioplegia Del Nido modificada anterógrada.

Resultados: Encaminhada ao CTI estável hemodinamicamente. No dia 14/10, observa-se instabilidade hemodinâmica, refratária à reposição volêmica, aumento de enzimas cardíacas e alterações eletrocardiográficas. Realizado ecocardiograma transtorácico que mostrou disfunção sistólica anterosseptal, com balonamento apical e hipercinesia basal, diante do que se iniciou terapia com balão intra-aórtico. Em 19/10, apresentava-se assintomática e com ecocardiograma evidenciando total recuperação da função ventricular. Cineangiocoronáriografia sem lesões coronarianas. Recebeu alta hospitalar no dia 25/10.

Conclusão: A síndrome de Takotsubo constitui uma forma de insuficiência cardíaca, reversível e classicamente precipitada por aflição emocional, mais comum em mulheres idosas. Neste caso evidenciamos o acometimento de uma mulher jovem, que sofreu estresse miocárdico devido à cirurgia cardíaca e uso de norepinefrina no pós operatório, com desfecho favorável. A suspeição diagnóstica é fundamental para o adequado manejo terapêutico desta condição clínica.

\section{PO 87}

\section{Formação de psudoaneurisma em aneurisma prévio do ventrículo esquerdo: um relato de caso}

Samuel Soares Eduardo, Paulo Marcelo Barbosa Mesquita, Andreza Sérvula, Alessandra Ribeiro de Albuquerque, Laíza Santos Leite Ribeiro

Introdução: A rotura do ventrículo esquerdo ocorre em até 4\% dos IAM. Raramente a rotura é contida, originando uma cavidade delineada por tecido fibroso, denominada pseudoaneurisma. Diferente do aneurisma verdadeiro, os falsos aneurismas apresentam grande risco de rotura e necessitam de correção cirúrgica urgente.

Objetivo: Relatar o caso de um pseudoaneurisma com ruptura da parede do ventrículo esquerdo.

Métodos: Estudo observacional descritivo, para descrever o caso de um paciente admitido no Hospital do Coração do Cariri. Realizado por meio de consulta ao prontuário hospitalar, imagens dos métodos diagnósticos e da cirurgia, e revisão da literatura.

Resultados: Homem de 73 anos, admitido em nosso serviço após consulta ambulatorial por dispneia aos esforços em junho de 2016. Histórico de infarto 1 ano antes, com eco antigo mostrando aneurisma de VE. Apresentava ECO recente mostrando pseudoaneurisma de VE e FE de 15\%. Foi admitido para preparação cirúrgica; CATE mostrou oclusão total proximal da DA. Seguiu para abordagem cirúrgica no dia seguinte. A circulação extracorpórea foi instalada por vasos femorais. Encontramos um grande aneurisma anterior de VE, com perfuração e formação de pseudo aneurisma com trombos. Optamos pela exclusão do aneurisma e correção geométrica da parede ventricular com patch de pericárdio bovino. A evolução foi favorável, recebendo alta no $8^{\circ}$ dia pós-operatório.

Conclusão: Embora muito grave, a ruptura do VE pode não ser fatal, evoluindo com pseudoaneurisma. A cirurgia deve ser realizada mesmo com disfunção grave do VE e é capaz de alterar de forma satisfatória os sintomas e a sobrevida desses pacientes. 


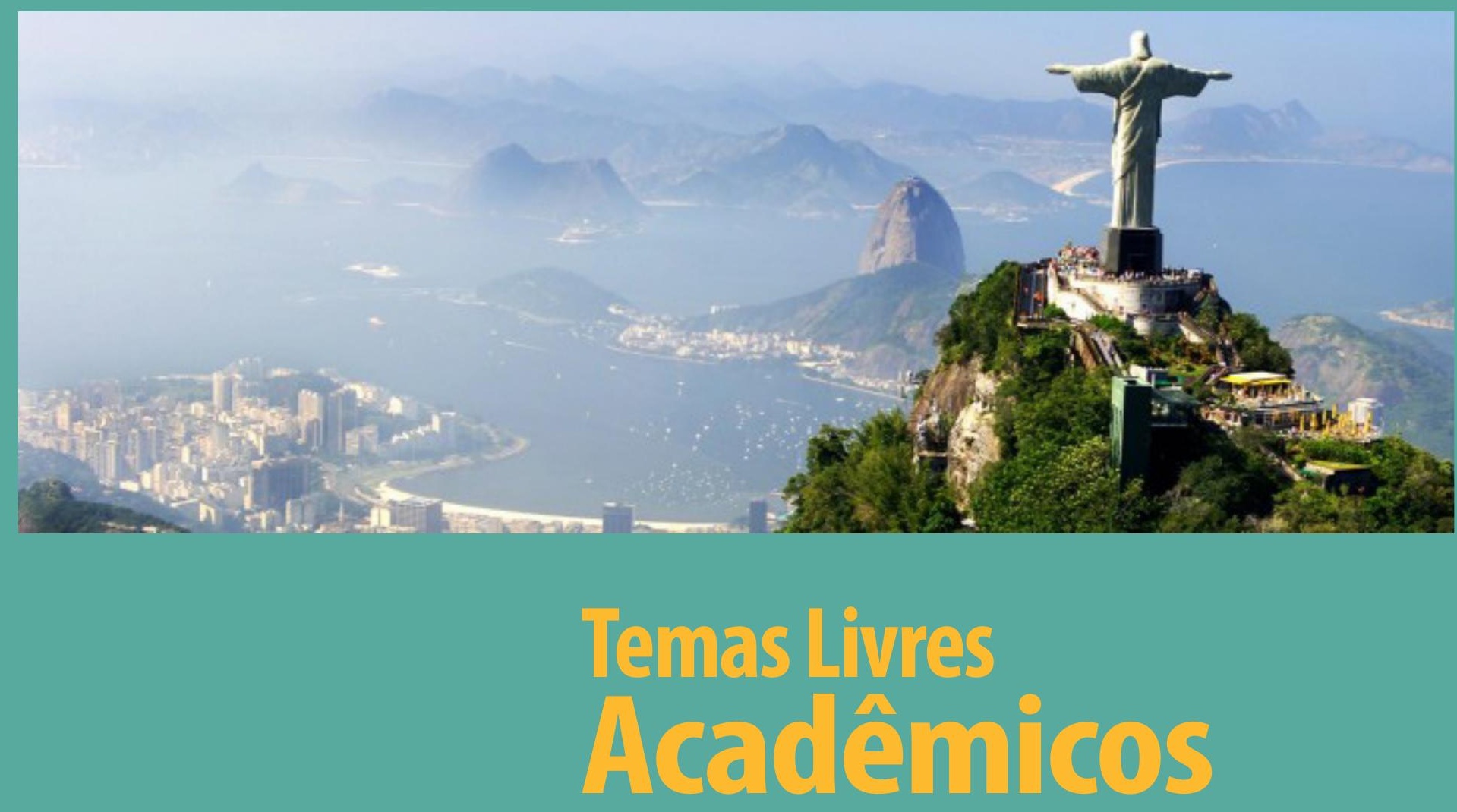


\section{TL $01 \bullet 8$ 8h45-9h}

\section{Impacto da hipertensão pulmonar pré-operatória nos desfechos de pacientes submetidos a cirurgia cardiovascular}

Armindo Jreige Júnior, Larissa Lucas Schloicka, Murilo Teixeira Macedo, Claudio Ribeiro da Cunha, Fernando Antibas Atik

Introdução: Pacientes submetidos a cirurgia cardiovascular com hipertensão pulmonar (HP) preexistente apresentam maior risco de complicações pós-operatórias.

Objetivo: Estudar, em pacientes submetidos a cirurgia cardiovascular, a frequência e o impacto da HP pré-operatória nos resultados imediatos do tratamento cirúrgico.

Métodos: Entre janeiro de 2006 e junho de 2014, 2.615 pacientes adultos com doenças cardíacas de indicação cirúrgica foram selecionados com base na presença de uma avaliação ecocardiográfica pré-operatória. A HP foi definida pela presença de pressão sistólica do ventrículo direito acima de $60 \mathrm{mmHg}$ pela equação de Bernoulli. A relação entre HP e desfechos de morbidade e mortalidade foi determinada por regressão logística.

Resultados: A HP foi identificada em 221 pacientes (8,45\%), principalmente nos submetidos a cirurgias valvares (22,6\%), sobre valva mitral $(25,7 \%)$ e reoperações (30,3\%). Os pacientes com HP eram mais jovens, predominantemente mulheres, em estado crítico no préoperatório (8,6\% contra 3,6\%, P=0,002), operações de emergência (6,6\% contra 3,2\%, $\mathrm{P}=0,02)$, graus mais avançados de classe funcional $(\mathrm{P}<0,0001)$, maior proporção de fibrilação atrial (41,7\% contra 10,7\%, $\mathrm{P}<0,0001)$ e maior pontuação do EuroSCORE $(7,1$ contra 2,8, $\mathrm{P}<0,0001)$. No pós-operatório, evoluíram com maior mortalidade $(P=0,01)$, maior taxa de insuficiência renal aguda $(P=0,004)$ e permanência mais longa em terapia intensiva e hospitalar ( $\mathrm{P}<0,001)$. Entretanto, a HP não foi fator independente de mortalidade na análise multivariada.

Conclusão: HP no pré-operatório de cirurgia cardiovascular é relativamente frequente, especialmente em reoperações valvares mitrais, estando associada a fatores comumente presentes em valvopatas crônicos de longa data. Apesar de não ser um fator independente de mortalidade, é uma variável que gera maior risco no pós-operatório, pela maior taxa de complicações.

\section{TL $02 \bullet 9 h-9 h 15$}

\section{Utilização de bomba centrífuga conectada à cânula implantável de longa duração como suporte circulatório pediátrico: desenvolvendo soluções economicamente viáveis}

Rafael Ceconi, Luiz Fernando Caneo, Aida Luiza Ribeiro Turquetto, Idagene A. Cestari, Helena T. T. Oyama, Ismar N. Cestari, Carla Tanamati, Juliano Gomes Penha, Leonardo Augusto Miana, Yarla Alves dos Santos, Marcelo Biscegli Jatene

Introdução: O transplante cardíaco pediátrico apresenta resultados favoráveis na insuficiência cardíaca avançada. O tempo de espera para o transplante, nas crianças com menos de $10 \mathrm{~kg}$, mostra-se superior a 300 dias em nosso país, 2 a 3 vezes superior à Europa e aos Estados Unidos. Dispositivos para o suporte de longo prazo são uma necessidade, porém seu custo é bastante elevado. Muitas dessas crianças apresentam-se em nossos hospitais em condições críticas (INTERMACS 1 ou 2), com prognóstico bastante reservado para implante direto desses dispositivos. O uso de bomba centrífuga conectada a uma cânula implantável de longa duração parece uma combinação atrativa, tanto em termos de segurança quanto em economia hospitalar.

Objetivo: Apresentar uma série de casos em que foi implantada uma cânula desenvolvida em nosso serviço, conectada a uma bomba centrífuga, como ponte para transplante, para recuperação ou para dispositivo de longa duração.

Métodos: Estudo retrospectivo de seis casos que utilizaram uma bomba centrífuga conectada a cânula de longa duração implantada cirurgicamente como resgate no choque cardiogênico, empregadas como ponte para recuperação, ponte para transplante ou ponte para ponte.

Resultados: O implante das cânulas mostrou-se tecnicamente factível, possibilitou adequada descompressão do ventrículo esquerdo, diminuiu a formação de trombos comparado às cânulas convencionais e garantiu grande mobilidade ao paciente.

Conclusão: A combinação de uma bomba centrífuga com a cânula implantável de longa duração permitiu adequado suporte circulatório mecânico ao paciente crítico. Foi facilmente explantada nos casos de recuperação da função cardíaca. Nos casos em que havia um dispositivo paracorpóreo disponível, a transição foi bastante simples. 


\section{APRESENTAÇÃO DE TEMAS LIVRES - Acadêmicos}

\section{TL $03 \cdot 9 h 15-9 h 30$}

\section{Impacto do desempenho técnico-cirúrgico nos resultados imediatos da cirurgia cardiovascular pediátrica: uma análise preliminar}

Yarla Alves dos Santos, Rafael Ceconi, Luiz Fernando Caneo, Aida Luiza Ribeiro Turquetto, Carla Tanamati, Leonardo Augusto Miana, Juliano Gomes Penha, Marcelo Biscegli Jatene

Introdução: A qualidade em cirurgia cardíaca pediátrica tem sido avaliada por meio dos resultados cirúrgicos obtidos, considerandose apenas fatores de risco e de complexidade para os diversos tipos de procedimentos. Entretanto, um resultado não favorável pode ocorrer em decorrência de uma técnica cirúrgica inadequada, uma falha no cuidado pós-operatório ou uma monitorização não otimizada, entre outros. Para medir o desempenho técnico-cirúrgico foi desenvolvida uma metodologia (Technical Performance Score - TPS) que avalia diferentes habilidades necessárias na correção cirúrgica de determinadas doenças.

Objetivo: O objetivo desse estudo foi avaliar o desempenho técnico-cirúrgico utilizando o TPS, e o impacto desse resultado no tempo de internação pós-operatória e na mortalidade imediata.

Métodos: Análise retrospectiva de 224 pacientes operados, com diagnóstico de comunicação interventricular (CIV). As cirurgias foram realizadas por um mesmo grupo cirúrgico, em um único hospital, e o desempenho cirúrgico foi classificado em ótimo, adequado e inadequado, por meio do TPS. O tempo de internação pós-operatória e a mortalidade hospitalar foram analisados em relação ao desempenho cirúrgico observado.

Resultados: Não houve diferença significativa entre os cirurgiões ( $P=0,053)$. Não ocorreu nenhum óbito na amostra estudada. $O$ desempenho técnico cirúrgico somente influenciou o tempo de internação no grupo de pacientes que necessitou de implante de marca-passo definitivo por bloqueio atrioventricular $(\mathrm{P}=0,008)$.

Discussão: A dissecção aguda da aorta (DAA) representa uma emergência cardiológica, com evolução rápida e fulminante. A mortalidade é alta, podendo atingir, antes da admissão, até 21,4 e 75\% nas primeiras 48 horas. Para que a intervenção seja adequada, é necessário um diagnóstico preciso e imediato.

Conclusão: O TPS permitiu uma análise bastante adequada do desempenho cirúrgico no tratamento da CIV. Não houve relação do desempenho técnico-cirúrgico com a mortalidade imediata. Pacientes que necessitaram de implante de marca-passo definitivo permaneceram internados por mais tempo.

\section{TL $04 \cdot 9 h 30-9 h 45$}

\section{Vasoplegia no pós-operatório imediato de cirurgia de revascularização do miocárdio: fatores preditivos e evolução}

Gustavo Farias Porciuncula, Débora Klein Ferreira, João Carlos Vieira da Costa Guaragna, Vera Elizabeth Closs, Luciano Cabral Albuquerque, Mário Wiehe, Luiz Carlos Bodanese, Rubens Lorentz de Araújo, Marco Antônio Goldani, João Batista Petracco, Daniela Santos Bandeira, Thiago Motta Netto, Andrio Coletto Bozzetto

Introdução: A síndrome vasoplégica (SV) é uma complicação grave no pós-operatório imediato (POI) de cirurgia cardíaca com CEC, com incidência entre 5 e 25\%, e que aumenta morbidade e mortalidade pós-operatórias, justificando o conhecimento de seus fatores de risco e da sua evolução.

Objetivo: Avaliar fatores preditivos e desfechos de SV no POI de cirurgia de revascularização miocárdica (CRM), por meio de um estudo de coorte prospectivo.

Métodos: Foram avaliados pacientes submetidos à CRM em hospital de Porto Alegre/RS, entre dezembro de 2004 e julho de 2015. A análise descritiva foi realizada por medidas de frequência, tendência central e dispersão. A associação entre variáveis categóricas foi realizada por meio do teste Qui-quadrado de Pearson e do Teste Exato de Fischer, e a comparação entre médias das medidas contínuas, pelo teste T de Student. A análise multivariada ocorreu por regressão logística binária. As análises foram realizadas considerando-se o nível de confiança de 95\% ( $a=5 \%)$. 
Resultados: Foram avaliados 3.897 pacientes submetidos à CRM, com média de idade de 61,3土9,9 anos (intervalo de 25 a 91 anos), a maioria do sexo masculino (67,5\%). Duzentos e onze pacientes (5,4\%) desenvolveram vasoplegia, com mortalidade de 13,7\%. Na análise multivariada, os fatores preditivos obesidade, betabloqueador e tempo de pinçamento $\geq 60$ min, e os desfechos sepse, ventilação mecânica e tempo de UTI prolongados, associaram-se à vasoplegia.

Conclusão: Em nossa análise, obesidade, uso de betabloqueador e tempo de pinçamento aórtico $\geq 60$ min associaram-se à maior ocorrência de SV. Pacientes com SV apresentaram pior curso pós-operatório, com sepse, ventilação mecânica e tempo de internação prolongados, o que acentuou morbidade e mortalidade.

\section{TL $05 \bullet 9$ 9h45-10h}

\section{Estudo sobre o coração univentricular e suas opções de tratamento}

Camylla Santos de Souza, Caroline Sbardellotto Cagliari, Bianca Alves de Miranda, Patrícia Fraga Paiva, Marília Abirachid Rezende, Leo Christyan Alves de Lima, Lívia Liberata Barbosa Bandeira, Thamires Politano de Sant'Anna Alves, Rodrigo Almeida Fontenele, Priscila Ferreira de Lima e Souza, Maurício Guagnini Boldo, João David de Souza Neto, Francisco Siosney Almeida Pinto, José Wanderley Neto

Introdução: No coração univentricular, ambos os átrios estão ligados a uma só câmara, seja pela ausência de uma das valvas atrioventriculares, seja pela presença das duas com drenagem para o mesmo ventrículo, o qual possui tamanho normal, sendo o outro hipoplásico.

Objetivo: Analisar tratamentos disponíveis para corações univentriculares, condição complexa cujo manejo desafia a medicina atual.

Métodos: Revisão sistemática de 13 artigos na plataforma MEDLINE.

Resultados: Aproximadamente 1\% dos recém-nascidos vivos são acometidos por malformações congênitas cardíacas, dentre as quais está o coração univentricular, com 25 casos descritos na literatura. $O$ tratamento cirúrgico ameniza a cianose induzida pela mistura do sangue venoso e arterial e a sobrecarga das câmaras, o que pode levar a dilatação, hipertrofia e insuficiência cardíacas. Na primeira fase do tratamento, logo após o nascimento, a cirurgia de Norwood/Sano, variação do procedimento de Blalock-Taussig, cria um shunt artéria pulmonar-aorta. Quatro meses depois, é realizada a fase de Glenn, criando uma ligação entre a veia cava superior e a artéria pulmonar, a fim de constituir um fluxo sanguíneo apropriado para os pulmões. Na terceira fase, de Fontan, liga-se a veia cava inferior à artéria pulmonar, para que o fluxo venoso adentre diretamente à artéria pulmonar e o coração apresente duas câmaras funcionais permanentemente. O transplante cardíaco permanece alternativa satisfatória; entretanto, apresenta dificuldades em relação às particularidades anatômicas de doadores neonatos.

Conclusão: O coração univentricular é condição rara, que compromete a sobrevivência dos recém-nascidos acometidos, sendo importante uma maior pesquisa de melhorias no tratamento cirúrgico de tal complicação. 


\title{
APRESENTAÇÃO DE TEMAS LIVRES - Acadêmicos
}

\section{TL $06 \bullet 14 \mathrm{~h} 30-14 \mathrm{~h} 45$}

\section{Quais as variáveis preditoras de mortalidade em reoperações valvares mitrais? Vinte anos de experiência de um único cirurgião}

\author{
Daniel Tomasi Keppen Sequeira de Almeida, Filipe Tomasi Keppen Sequeira de Almeida, Rui M. S. Almeida
}

Introdução: Dentre as doenças cardíacas, as doenças valvares mitrais são das mais prevalentes. Sem um substituto valvar ideal, os pacientes têm de ser submetidos a reoperações ao longo da vida.

Objetivo: Os autores avaliam retrospectivamente uma série consecutiva de reoperações da valva mitral e identificam as variáveis, estatisticamente significativas, preditoras de mortalidade, por análise multivariada.

Métodos: Foram obtidos dados, de fevereiro de 1993 a novembro de 2016, de 105 pacientes, operados por um único cirurgião, e submetidos a reoperação valvar mitral. Os dados demográficos incluíram dados clínicos e ecocardiograficos pré-operatórios, variáveis trans e pós-operatórias, sendo todos analisados por meio de análise multivariada, para prever os que podem interferir na mortalidade hospitalar. Os pacientes foram divididos, de acordo com o EuroScore logística, em três grupos (0-4,9\%, 5-9,9\% e 10\% e acima).

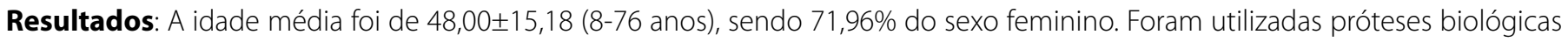
em 70,09\%, mecânicas em 23,36\% e realizado reparo em 6,54\%. Procedimentos associados foram realizados em 9,35\%, sendo a substituição da valva aórtica concomitante realizada em 50\% e a revascularização do miocárdio em 30\%. O apêndice atrial esquerdo foi fechado em todos os casos em que a fibrilação atrial era o ritmo de base (56,07\%). O EuroScore logístico médio foi

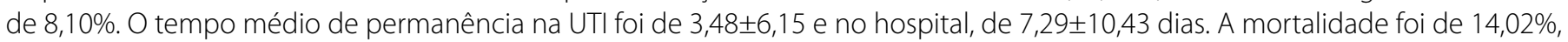
principalmente no grupo de alto EuroScore, e com mais de uma reoperação.

Conclusão: A análise estatística identificou idade, classe funcional e hipertensão pulmonar primária como preditores préoperatórios para mortalidade.

\section{TL $07 \bullet 14 h 45-15 h$}

\section{Endocardite infecciosa em cirurgias valvares - avaliação dos critérios ecocardiográficos e clínicos como preditores de mortalidade em uma serie de casos}

Igor Arantes de Oliveira Góes, Gustavo Adolfo Kuriyama Massari, Cristina de Sylos, Leonardo Moraes de Albuquerque, Mário Issa, Thierry Araújo, Romulo Bonini

Introdução: Apesar dos avanços no diagnóstico, na terapêutica e nas técnicas cirúrgicas, a incidência da endocardite infecciosa (El) não regrediu nos últimos 30 anos, com alta mortalidade intra-hospitalar (10-26\%).

Objetivo: Analisar uma série de casos diagnosticados como El e operados em hospital terciário, comparando critérios clínicos e ecocardiográficos com a literatura mundial.

Métodos: Estudo retrospectivo observacional, com análise de prontuários dos pacientes operados no serviço diagnosticados como El no período entre janeiro de 2015 e outubro de 2016.

Resultados: Das 271 cirurgias cardíacas realizadas nesse período, 57 tinham origem valvar, sendo 8 por El (14\%). Houve prevalência do gênero masculino (62,5\%), com idade média de 41 anos. Setenta e cinco por cento das valvas afetadas eram nativas, sendo a aórtica a mais acometida (62,5\%). A média de tempo sintomas-diagnóstico foi de 3,66 dias, sendo febre e dispneia os sintomas mais comuns (37,5\%). Ao ecocardiograma, 62,6\% dos pacientes apresentavam vegetações maiores que 10 mm, e disfunção valvar importante em $75 \%$ dos casos, predominando insuficiência. Todos os casos utilizaram associação de antibióticos, em que a gentamicina esteve presente em metade deles. A mortalidade intra-hospitalar na amostra ocorreu em 3 pacientes, um com disfunção ventricular, outro com sepse e outro com AVC extenso e sepse.

Conclusão: Observamos alta incidência de El, com elevada mortalidade não relacionada ao procedimento cirúrgico em si, mas a complicações neurológicas, disfunção ventricular e sepse. Ressalta-se a necessidade de intervenção precoce com a intenção de reduzir complicações como dilatação, disfunção ventricular e embolias. 


\section{TL $08 \bullet 15 h-15 h 15$}

\section{Influência da impressão 3D na Cirurgia cardiovascular}

Caio Teixeira dos Santos, Camylla Santos de Souza, Thais Lemos de Souza Macêdo, Bianca Alves de Miranda, Matheus Henrique Seixas dos Santos, Jonathan Augusto Venceslau Lima, Matheus Jorge Pires Viana, Alice Albuquerque Figueiredo, Guilherme Nobre Cavalcanti Lucas, Victória Queiroz Ramos, Igor Rodrigues da Silva, Lara Ferreira Ventura, Ana Carolina Vieira

Introdução: A impressão 3D conquistou um espaço relevante na cirurgia cardiovascular, por possibilitar intervenção em casos de alto risco e/ou inoperáveis, bem como treinamento e ensino de médicos, residentes e estudantes.

Objetivo: Apresentar a influência e os benefícios da impressão 3D na cirurgia cardíaca.

Métodos: Revisão sistemática da literatura, utilizando-se as bases de dados NCBI, BVS e MEDLINE.

Resultados: A impressão 3D tem elevado a eficiência de cirurgias cardiovasculares e da descoberta de diagnósticos. Por possibilitar um planejamento minucioso das etapas operatórias, proporciona maior segurança para o paciente, diminuindo a morbidade e mortalidade, o tempo das cirurgias e o risco de complicações. Alguns pré-procedimentos cardíacos já estão utilizando impressões 3D para auxiliar no estudo de casos, como tumores cardíacos, válvula aórtica bicúspide, tetralogia de Fallot, aneurismas ventriculares ou casos que serão submetidos a transplantes de coração univentricular, implantes valvares aórticos transcateter, substituições de válvulas mitrais, entre outros. No Brasil, a ANVISA ainda não libera o uso cirúrgico de implantes personalizados e o emprego de tecnologias 3D necessita de uma autorização especial. O Centro de Tecnologia da Informação Renato Archer (CTI) - Campinas, é a entidade brasileira que presta esse serviço aos hospitais nacionais. Desde 2000, o CTI produziu cerca de 3.385 peças para cirurgias e, nos últimos três anos (2015), o CTI tem auxiliado em aproximadamente 500 cirurgias.

Conclusão: $\mathrm{O}$ avanço da impressão 3D em cirurgias de difícil visualização qualifica os treinamentos práticos de médicos e residentes, possibilitando melhores prognóstico e intervenção. 


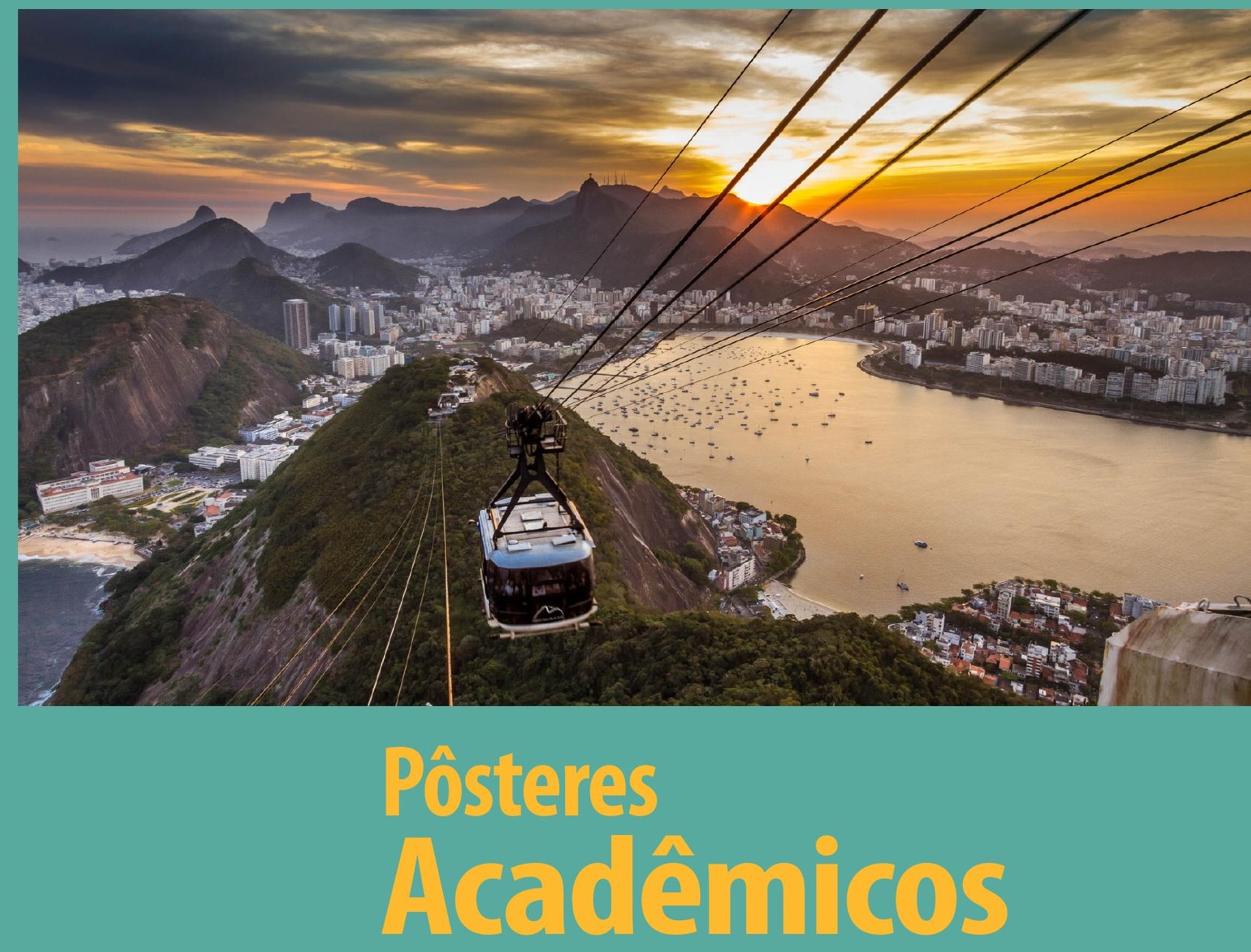


SESSÃO I

21 de abril • sexta-feira
SALA DE PÔSTERES

$10 \mathrm{~h}-10 \mathrm{~h} 45$
EXPOSIÇÃO DE PÔSTERES

- Acadêmicos

PO 01

\section{Análise dos resultados pós-cirúrgicos de inovações em procedimentos percutâneos de trocas valvares: TAVI e MitraClip}

Camylla Santos de Souza, Patrícia Fraga Paiva, Ana Carolina Ramos Queiroz, Sarah Araujo Loss, Luana Menezes Agostinho, Bianca de Negri Souza, Osmar Mendes Peixoto Filho, Victor Valente Lopes, Marlon Moreira Nery, Raphaella von Sohsten Calabria Lima, Marília Abirachid Rezende, Kaio Cezar Ferreira, André Rainho, Ana Letícia Luchiari Ferrari, Thais Lemos de Souza Macêdo, João David de Souza Neto, Sérgio Francisco dos Santos Júnior

Introdução: Em virtude do maior risco de complicações da cirurgia valvar convencional em pacientes idosos e de alto risco operatório, as técnicas percutâneas vêm se tornando tratamento indicado neste grupo, melhorando a sobrevida.

Objetivo: Avaliar dois dos procedimentos percutâneos mais utilizados para tratar as duas valvulopatias mais comuns: implante de valva aórtica transcateter (TAVI, para estenose aórtica) e MitraClip ${ }^{\circledR}$ (para insuficiência mitral).

Métodos: Análise de dados de pacientes e revisão de 54 artigos na MEDLINE.

Resultados: Desde 2008, mais de 2.000 TAVIs foram realizados por cerca de 100 médicos em 126 hospitais no Brasil, que abriga 7 centros de treinamento. A mortalidade foi inferior à da troca valvar convencional (3,4\% vs. 6,5\%), e os índices de AVC são menores no pós-operatório e após 1 ano de TAVI (8,8\% vs. 12,6\% na cirurgia aberta). A sobrevida também em 1 ano é superior (85,8\%). A experiência do cirurgião conta na escolha da abordagem (se femoral, transapical, subclávia, transaórtica, transaxilar ou transcaval), atentando para complicações como lesão vascular, vazamentos paravalvulares e necessidade de marca-passo. O MitraClip obteve 99,6\% de sucesso no estudo ACCESS-EU, com sobrevida de 81,8\%. As complicações e a permanência hospitalar com o MitraClip ${ }^{\circledR a ̃ o}$ menores que na cirurgia convencional. Houve melhora completa da insuficiência mitral em quase 80\%, com NYHA passando para classe I/II.

Conclusão: O TAVI e o MitraClip ${ }^{\oplus}$ são técnicas em franca evolução, em virtude de sua alta eficácia, menos complicações e rápida recuperação.

\section{PO 02}

\section{Assistência circulatória mecânica em pacientes com choque cardiogênico refratário, sem história prévia de disfunção miocárdica. Experiência inicial de um serviço}

Diogo Assis Souza, Felipe Bruno Santos da Cunha, Henrique Louzan Machado, Rafael Ramos Amaral, Leonardo Jadyr Silva Rodrigues Alves, Thiago do Amaral Cavalcante, Felipe Duarte Moraes, Ricardo Barros Corso, Isaac Azevedo Silva, Marcus Vinicius Nascimento Silva, Glauco Pina, Helmgton J. B. Souza

Introdução: O choque cardiogênico é a forma mais severa de falência cardíaca aguda. A assistência circulatória mecânica (ACM) vem conquistando espaço no tratamento da IC.

Objetivo: Apresentar a experiência inicial do Programa de Assistência Circulatória Mecânica, em pacientes diagnosticados com choque cardiogênico refratário, sem evidência de disfunção miocárdica prévia.

Métodos: Pacientes submetidos a ACM entre agosto de 20114 e fevereiro de 2016, com choque cardiogênico refratário (classificados como INTERMACS 1) e sem história prévia de disfunção miocárdica. Trata-se de estudo de série de casos. Os dados numéricos estão expressos em média, desvio padrão e valor mínimo/máximo.

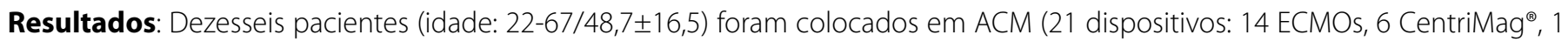
Impella ${ }^{\circledast}$ ). Destes, 10 foram diagnosticados com choque cardiogênico refratário, sendo 7 sem história prévia de disfunção miocárdica

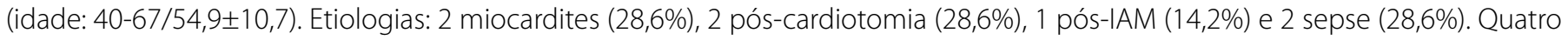
foram tratados com ECMO, 1 com CentriMag ${ }^{\oplus}$ e 1 com Impella ${ }^{\oplus}$. Um paciente foi assistido com ECMO, evoluindo para CentriMag ${ }^{\oplus}$. O tempo de assistência variou entre 12 horas e 9 dias (5,4 43$)$. Cinco pacientes recuperaram a função ventricular (71,4\%). Ocorreram 2 óbitos durante a assistência (28,6\%), um por hemorragia e outro por sepse.

Conclusão: Na série apresentada, a assistência circulatória mostrou-se efetiva no tratamento de pacientes com choque cardiogênico, possibilitando a recuperação da função miocárdica. 


\title{
EXPOSIÇÃO DE PÔSTERES \\ - Acadêmicos
}

\section{PO 03}

\section{Manejo cirúrgico de crianças portadoras de ventrículo direito com dupla câmara: experiência de Alagoas}

Lucas Roberto da Silva Barbosa, Kamila Maria Quixadá Lira, Rany de Andrade Amaral, Isabela Gomes Alves Munhoz, Thais Eigler Lima, Eduarda Cavalcante Santana, Laio Caju Wanderley, José Wanderley Neto, José da Silva Leitão Neto

\begin{abstract}
Introdução: O ventrículo direito com dupla câmara (VDDC) se caracteriza por bandas musculares anômalas hipertróficas que dividem a cavidade ventricular em uma porção proximal (alta pressão) e outra distal (baixa pressão). Os exames pré-operatórios ocasionalmente não demonstram a condição real do paciente, levando a achados cirúrgicos.
\end{abstract}

Objetivo: Descrever a experiência em um hospital de referência do estado de Alagoas, as condutas adotadas para o diagnóstico e resolução da condição, e discutir sobre as dificuldades do diagnóstico.

Métodos: Foram analisados, retrospectivamente, os registros de todos os pacientes diagnosticados e submetidos à correção de VDDC.

Resultados: A faixa etária variou de 4 a 15 anos, predominando o sexo masculino. Foram submetidos à ventriculoseptoplastia e ressecção de banda anômala em ventrículo direito e bandas secundárias. Apresentaram comunicação interventricular (CIV) com dimensões de até $1,5 \mathrm{~cm}$, corrigidas com sutura direta ou pericárdio bovino. Um dos pacientes, ao final da circulação extracorpórea (CEC), apresentou bloqueio atrioventricular intermitente, sendo tratado com marca-passo. Outro apresentou forame oval patente ao ecocardiograma, e em cirurgia foi constatado forame oval fechado.

Discussão: A incidência da condição varia de 0,5\% a 2\% e em $80 \%$ a $90 \%$ dos casos está associada com CIV, porém, no presente estudo, a associação foi de 100\%. Outro aspecto relevante foi o achado cirúrgico em um paciente que no exame pré-operatório não havia sido diagnosticado com VDDC.

Conclusão: Ainda que VDDC seja uma condição rara e pouco abordada na literatura, as intervenções cirúrgicas adotadas em seu tratamento se mostraram eficazes e de sucesso. Deve-se ainda atentar para as possíveis variações no diagnóstico em razão dos sintomas inespecíficos.

\section{PO 04}

\section{Suporte circulatório mecânico (SCM) em pacientes com SARA - relatos de série de casos}

Rafael Ramos Amaral, Thiago do Amaral Cavalcante, Diogo Assis Souza, Henrique Louzan Machado, Leonardo Jadyr Silva Rodrigues Alves, Felipe Bruno Santos da Cunha, Felipe Duarte Moraes, Helmgton J. B. Souza

Introdução: O uso de suporte respiratório extracorpóreo reduz a mortalidade em até 89\% dos pacientes com insuficiência respiratória refratária secundária à síndrome do desconforto respiratório agudo (SARA).

Objetivo: Relatar a evolução de três pacientes, submetidos à SCM-ECMO WV, com canulação periférica, para tratamento de Insuficiência respiratória refratária, secundária à SARA.

Métodos: Caso 1: RMA, masculino, 33 anos, previamente hígido, usuário de drogas, admitido após tentativa de autoextermínio com insuficiência respiratória. Evoluiu, ITR e SARA, não responsivo às manobras de recrutamento e terapia de prona (pH: 7,2/ pO2: 93 mmHg/pCO2: 105 mmHg). Indicado SCM com ECMO-V, permanecendo em assistência por 8 dias, com recuperação do parênquima pulmonar. Caso 2: TVR, 37 anos, diagnosticado com leptospirose e evoluindo com insuficiência respiratória refratária (SARA) e disfunção múltipla de órgãos (pH: 7,2/pO2: 75/pCO2: 105). Colocado em ECMO-VV, evoluiu com recuperação da função respiratória, sendo retirado de assistência após 5 dias.

Resultados: Caso 3: RMM, 22 anos, com diagnóstico de linfoma de Hodgkin e passado de transplante de medula, quimioterapia e radioterapia. Cursou com insuficiência respiratória e SARA refratária (pH: 7,15; pO2: 68; pCO2: 58), apesar de parâmetros ventilatórios máximos (FiO2: 100\%; PEEP: 14), instabilidade hemodinâmica e falência de múltiplos órgãos. Colocado em SCM (ECMO-W) por seis dias, apresentando recuperação da função respiratória, com desmame e explante do dispositivo.

Conclusão: Nos casos apresentados, o uso de SCM-ECMO foi eficaz no tratamento de insuficiência respiratória refratária, secundária à SARA, possibilitando recuperação orgânica e sobrevida dos pacientes. 
SESSÃO II

21 de abril • sexta-feira
SALA DE PÔSTERES

16h-16h45
EXPOSIÇÃO DE PÔSTERES

- Acadêmicos

\section{PO 05}

\section{Suporte circulatório mecânico em pacientes com sepse - série de casos}

Henrique Louzan Machado, Thiago do Amaral Cavalcante, Leonardo Jadyr Silva Rodrigues Alves, Diogo Assis Souza, Rafael Ramos Amaral, Felipe Bruno Santos da Cunha, Felipe Duarte Moraes, Helmgton José Brito de Souza

Introdução: Suporte circulatório mecânico (SCM) tem se mostrado eficaz no tratamento de doenças respiratórias e cardiovasculares graves, quando as estratégias para manter o funcionamento orgânico se mostram insuficientes. O uso de oxigenação por membrana extracorpórea (ECMO) para tratamento de choque cardiogênico secundário à depressão miocárdica por sepse ainda é controverso.

Objetivo: Relatar o caso de dois pacientes, sem cardiopatia prévia, colocados em SCM com ECMO para tratamento de choque cardiogênico secundário a sepse.

Métodos: Caso 1:VMQ, feminino, 42 anos, diagnosticada com tumor abdominal recidivado, submetida a exérese e evoluindo com abcesso retrogástrico, drenado cirurgicamente. Extubada no PO, evoluiu com insuficiência respiratória, rebaixamento sensorial, hipotensão, acidose metabólica importante (pH: 7,0/HCO3: 9,3) - INTERMACS 1. Eco transtorácico mostrou disfunção biventricular severa (FE: XX\%), sendo indicada ECMO-VA. Houve reversão plena da acidose e progressiva redução de aminas. Após X dias, paciente apresentou recuperação total da disfunção miocárdica, sendo possíveis o desmame e o explante do dispositivo.

Resultados: Caso 2: ACFF, feminino, 40 anos, com gestação gemelar, evoluindo com abortamento séptico e histerectomia de emergência. Evoluiu com choque, acidose refratária e disfunção orgânica múltipla. Eco mostrou disfunção ventricular esquerda (FE: 18\%). Colocada em ECMO-VA, evoluiu com melhora hemodinâmica, desmame de aminas e correção da acidose. Após o sexto dia de assistência, apresentou recuperação da função ventricular, sendo possíveis o desmame e o explante do dispositivo.

Conclusão: Nos casos apresentados, o SCM se mostrou eficaz no tratamento da depressão miocárdica após sepse grave.

\section{PO 06}

\section{Novas tecnologias na cirurgia de troca de válvula aórtica}

Thaís Lemos de Souza Macêdo, Caio Teixeira dos Santos, José Hiago de Freitas Damião, Lívia Motta Leitão, Camylla Santos de Souza, Bianca Alves de Miranda, Marlon Moreira Nery, Matheus Henrique Seixas dos Santos, Jonathan Augusto Venceslau Lima, Thamires Politano de Sant'Anna Alves, Daniele Rosa Fraga, Ana Luiza Brum Reis Soares, Ana Claudia Ferreira Neves, Osmar Mendes Peixoto Filho, Bianca de Negri Souza, Igor Caio Alfena Arakaki, Leo Christyan Alves de Lima

Introdução: Apesar de evidências favoráveis à abordagem convencional em cirurgias de válvula aórtica, as novas tecnologias estão contribuindo para o desfecho final. A intervenção por implante transcateter de válvula aórtica (TAVI) e o procedimento da impressão em três dimensões (3D) são ferramentas úteis para cirurgiões cardiovasculares.

Objetivo: Identificar o estado da arte sobre tecnologia na cirurgia aórtica por meio de revisão da literatura.

Métodos: Análise dos recentes artigos de revisão no banco de dados NCBI, com os termos "3D printing", "cardiovascular surgery" e "TAVI". Incluídos somente artigos de cirurgia aórtica.

Resultados: Em pacientes idosos que não teriam condições hemodinâmicas para serem submetidas a uma toracotomia, o TAVI pode ser um método mais adequado. A tecnologia 3D mais utilizada e barata é Fused Deposition Modeling; a impressão dura 3-4 horas para réplicas moles e 5-7 horas para as rígidas. Para criação do objeto, é necessário um modelo virtual a partir de imagens volumétricas em 3D de alta qualidade. A impressão, com estruturas anatômicas fidedignas aos pacientes, pode superar as limitações dos exames de imagens, por permitir uma compreensão da anatomia tridimensional em casos complexos e raros, essencial para o planejamento perioperatório e a tomada de decisão.

Conclusão: O TAVI é alternativa terapêutica em pacientes de alto risco e outras comorbidades; já a tecnologia 3D oferece mais informações que os métodos gráficos convencionais além das réplicas customizadas individuais. As novas tecnologias melhoraram a eficácia terapêutica e o prognóstico e redução de complicações pós-operatórias. 


\section{EXPOSIÇÃO DE PÔSTERES - Acadêmicos}

\section{PO 07}

\section{Ruptura aguda da parede ventricular esquerda: um relato de caso}

Vinicius Vasconcelos do Lago, Miriã Almeida dos Santos, Alexandre José dos Santos Calasans, Sérgio Rodrigues Maranha

Introdução: Soares et al. ressaltam que a ruptura de parede livre de ventrículo esquerdo (RPLVE) é uma complicação que ocorre em $4 \%$ dos pacientes após infarto agudo do miocárdio (IAM). A ruptura aguda é caracterizada pela recorrência súbita de precordialgia, dissociação eletromecânica, choque e morte por sangramento no saco pericárdico e, nessa concepção, os autores ressaltam para a abordagem precoce.

Objetivo: O objetivo deste estudo é apresentar um relato de caso descritivo da condição apresentada pelo paciente, a ruptura aguda de parede ventricular esquerda.

Métodos: As bases de dados consultadas foram PUBMED, SCIELO, LILACS, MEDLINE e SCIENCEDIRECT.

Resultados: A RPLVE é a terceira complicação mais frequente do IAM. Apresenta-se com manifestações de tamponamento cardíaco e choque cardiogênico, associados a dor torácica intensa e hipotensão arterial sistêmica. O sinal clínico mais fidedigno de RPLVE é a dissociação eletromecânica. O mais importante exame complementar é o ecocardiograma. Representa uma emergência cirúrgica, especialmente quando é aguda; com frequência, o diagnóstico não é estabelecido em tempo suficientemente hábil para salvamento. As técnicas clássicas têm sido substituídas pela correção com retalho epicárdico bovino e utilização de cola biológica.

Conclusão: Concluímos que a rotura de parede livre é a mais grave complicação do IAM e as manifestações clínicas apresentadas pelo paciente são variadas, dependendo sobretudo do local da lesão. Estudos mostraram que a RPLVE aguda costuma ter elevado índice de mortalidade, independentemente da precocidade do diagnóstico. O tratamento é cirúrgico e a intervenção tem de ser rápida e precisa. 



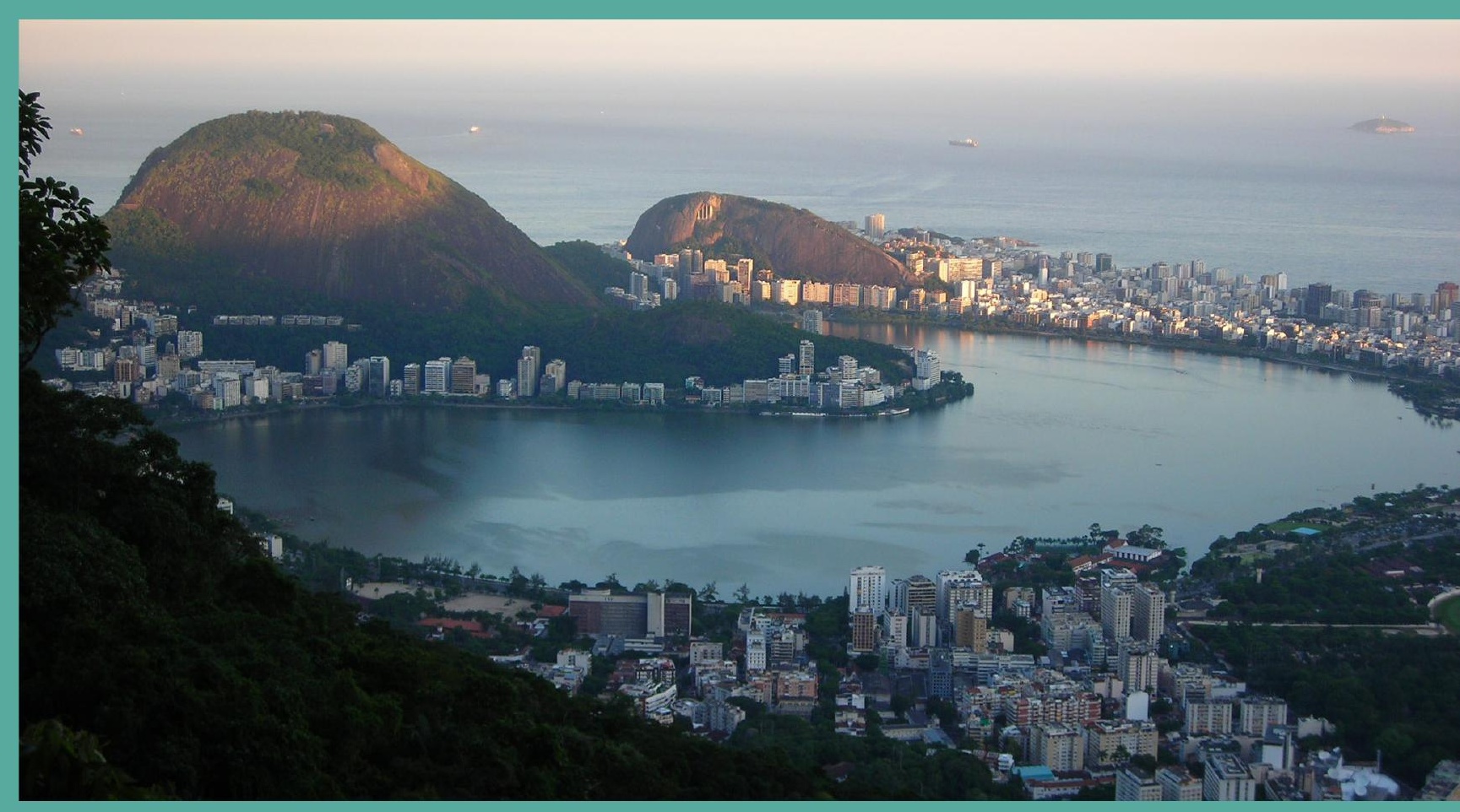

\section{Temas Livies}

Pediatria 


\section{Reintervenções durante o curso de ECMO em pacientes pediátricos e portadores de cardiopatias congênitas}

Leonardo Augusto Miana, Leandro Batisti de Faria, Santiago Raul Arrieta, Germana Coimbra, Aida Turquetto, Luiz Fernando Caneo, Carla Tanamati, Juliano Gomes Penha, Maria Aparecida Batistão, Vanessa A. Guimarães Borges, Filomena Regina B. Galas, Marcelo Biscegli Jatene

Introdução: A oxigenação por membrana extracorpórea (ECMO) é uma importante ferramenta de suporte circulatório mecânico para pacientes que evoluem com falência cardiopulmonar. Frequentemente, reintervenções diagnósticas e/ou terapêuticas são necessárias durante o curso da ECMO.

Objetivo: Descrever os resultados das reintervenções cirúrgicas ou por hemodinâmica realizadas durante suporte por ECMO em pacientes pediátricos e/ou portadores de cardiopatias congênitas.

Métodos: Estudo retrospectivo entre janeiro de 2012 e outubro de 2015 com 78 pacientes pediátricos e portadores de cardiopatias congênitas submetidos a suporte com ECMO. Dezessete pacientes $(21,8 \%)$ necessitaram de reintervenções. Foram estudados os procedimentos necessários e o impacto desses procedimentos no resultado de desmame da assistência e alta hospitalar.

Resultados: Dos 17 procedimentos, sete $(41,2 \%)$ foram intervenções hemodinâmicas. Duas angioplastias em artérias pulmonares, 1 stent em aorta, 1 atriosseptostomia, 1 dilatação de seio coronariano, 1 angioplastia coronariana e 1 embolização de sequestro. Houve uma lesão da artéria pulmonar após angioplastia com stent corrigida. Dez intervenções cirúrgicas (58,8\%) foram realizadas, sendo 2 para trombectomias, 3 para reposicionamento/inserção de cânulas e 5 abordagens cirúrgicas em lesões residuais (1 bandagem pulmonar, 1 Glenn, 1 retroca mitral e 2 correções de CIV residual e plastia tricúspide). O tempo de assistência foi 262 181,2 horas nos pacientes que necessitaram de reintervenção, 11 (64,7\%) puderam ser decanulados (off-ECMO) e 5 (29,4\%) obtiveram alta hospitalar, resultados estatisticamente semelhantes aos do grupo sem intervenção ( $P>0,05)$.

Conclusão: Reintervenções em ECMO, incluindo cateterismo cardíaco, são procedimentos que podem ser realizados com segurança em pacientes sob suporte circulatório com ECMO, permitindo o diagnóstico de lesões residuais e também podendo ser utilizadas como intervenção terapêutica.

\section{TL $02 \bullet 14 h 15-14 h 30$}

\section{O uso da mini-CEC em reoperações de tetralogia de Fallot minimiza a perda sanguínea quando comparada com o circuito de CEC convencional}

Leonardo Augusto Miana, Luiz Fernando Caneo, Alexandre Andrade, Maria Raquel Massoti, Guilherme Viotto, Elísio Rebouças, Aida Turquetto, Carla Tanamati, Juliano Gomes Penha, Karina Moreira, Sandra Regina Ormenesi, Cristiane Aquino, Filomena R. B. G. Galas, Marcelo Biscegli Jatene

Introdução: O circuito fechado de perfusão minimizado (Mini-CEC) tem sido demonstrado com sucesso nos procedimentos de revascularização do miocárdio. Este circuito tem a vantagem de minimizar o contato do sangue com superfícies não endotelizadas, aumentando a preservação dos elementos sanguíneos.

Objetivo: Avaliar a aplicabilidade e a segurança da mini-CEC em cirurgias para troca da válvula pulmonar (TVP) em reoperação de tetralogia de Fallot.

Métodos: Estudo retrospectivo. Quinze pacientes foram submetidos a TVP no período de janeiro de 2013 a dezembro de 2014. Cinco pacientes usaram o circuito de mini-CEC e os demais utilizaram CEC convencional. Todos os pacientes foram operados em normotermia e sem anóxia miocárdica. As características demográficas foram semelhantes nos dois grupos. Foram comparados os valores de hematócrito no pré-operatório, durante a CEC, após a CEC e no pós-operatório.

Resultados: Os pacientes do grupo mini-CEC apresentaram menos diminuição do hematócrito durante a operação $(47,8,47,40,5$, 43,0, 42,5 e 45,3\%) em comparação com os pacientes do grupo CEC convencional (45,1,42,4,34,4, 31,5, 33,5 e 34,2\%; P=0,004). Apenas 2 pacientes receberam transfusão sanguínea, ambos do grupo CEC convencional. Não houve óbito hospitalar.

Conclusão: Neste estudo preliminar, o circuito de mini-CEC demonstrou melhor preservação dos níveis de hematócrito comparado à CEC convencional. 


\section{APRESENTAÇÃO DE TEMAS LIVRES - Pediatria}

\section{TL $03 \bullet 14 \mathrm{~h} 30-14 \mathrm{~h} 45$}

\section{Resultados da conversão de operação de Fontan-Kreutzer (FK) para cavopulmonar total com tubo extracardíaco (CPT) por falência da circulação univentricular}

Gabriel Carmona Fernandes, Guilherme Viotto Rodrigues da Silva, Luiz Fernando Caneo, Carla Tanamati, Aída Luiza Ribeiro Turquetto, Marcelo Biscegli Jatene

Introdução: A FK, em que se realizava a conexão direta do átrio direito à artéria pulmonar, foi muito realizada no início dessa experiência, porém evolui a longo prazo com comorbidades importantes, como arritmias e enteropatia perdedora de proteína (EPP), causando piora na classe funcional (CF) desses pacientes. Nestes casos, a conversão para CPT pode ser um tratamento adequado para estas arritmias.

Objetivo: Avaliar os resultados da conversão da FK para a CPT.

Métodos: Trabalho retrospectivo de um único centro, em que foram analisados pacientes que realizaram a conversão de FK para CPT no período de 1995 a 2014 por meio da análise de prontuário.

Resultados: Foi realizado um total de 420 operações do tipo Fontan no período acompanhado; destas, 18 foram Fontan-Kreutzer e 10 foram convertidos para CPT. Houve 2 óbitos. A sobrevida atuarial de 5 e 10 anos foi de 80 e 75\%, respectivamente. Em 9 casos foi indicada por arritmia de difícil controle e 1 por enteropatia perdedora de proteína. Antes da conversão, 3 encontravam-se em CF I e 7 em CF II ou superior. Após a conversão, 80\% evoluíram com melhora da CF ( $P<0,05)$. Houve resolução de 57\% das arritmias. O tempo médio de internação na UTI foi de 13 dias, e hospitalar de 37 dias. Atualmente 1 paciente encontra-se em avaliação para transplante cardíaco.

Conclusão: Esta conversão cirúrgica é um procedimento complexo, com mortalidade e morbidade elevadas e tempo de internação prolongado; portanto, necessita ser realizada em hospitais terciários experientes. Apesar de uma amostra pequena, observa-se melhora na CF e resolução satisfatória das arritmias.

\section{TL $04 \cdot 14 \mathrm{~h} 45-15 \mathrm{~h}$}

\section{Tratamento cirúrgico da anomalia de Ebstein: resultados em longo prazo}

Guilherme Viotto Rodrigues da Silva, Leonardo Augusto Miana, Luiz Fernando Caneo, Aída Luiza Ribeiro Turquetto, Carla Tanamati, Juliano Gomes Penha, Elizio de Souza Robouças, Maria Raquel Massoti, Marcelo Biscegli Jatene

Introdução: A anomalia de Ebstein (EA) é uma cardiopatia congênita cianogênica rara, caracterizada por variáveis graus de inserção anômala dos folhetos septal e posterior da valva tricúspide em direção ao ápice do ventrículo direito (VD). Os objetivos do tratamento cirúrgico são a reconstrução do aparato valvar e a correção da insuficiência tricúspide (IT) e das malformações associadas.

Objetivo: O objetivo deste estudo foi avaliar os desfechos em longo prazo de pacientes submetidos à correção cirúrgica da EA.

Métodos: Estudo retrospectivo, que incluiu 62 pacientes submetidos à correção cirúrgica de EA. Foram avaliados os resultados em longo prazo: sobrevida, necessidade de reoperações, graus de regurgitação tricúspide (IT) e disfunção ventricular pós-operatória.

Resultados: Foi realizada plastia valvar isolada em 46 pacientes (12 casos - cirurgia do cone). A média de idade na cirurgia foi de $20,5 \pm 14,9$ anos. O tempo médio de seguimento foi de 8,27 $\pm 4,97$ anos. A mortalidade em 30 dias foi 8,3\% e a sobrevida em 1 ano e 10 anos foi de 93,3\% para ambos. Onze (17,7\%) pacientes necessitaram de reoperação, em um tempo médio de 4,5 $\pm 4,2$ anos após a primeira cirurgia. Quando se comparou presença de IT importante pós-operatória e o tipo de cirurgia realizada, pacientes submetidos à cirurgia do cone apresentaram menor incidência de IT importante quando comparados aos submetidos à plastia $\operatorname{valvar}(P=0,011)$.

Conclusão: Resultados em longo prazo no tratamento cirúrgico da anomalia de Ebstein demonstram sobrevida aceitável e baixa necessidade de reintervenções, e pacientes submetidos à reconstrução cônica da valva tricúspide apresentaram menor incidência de IT importante pós-operatória. 


\section{TL $05 \cdot 14 h-14 h 15$}

\section{Cirurgia cardíaca minimamente invasiva para tratamento da cardiopatia congênita no adulto}

Jerônimo Antonio Fortunato Junior, Jeferson R. Sesca, Wilson Nkundumukiza

Introdução: A cirurgia cardíaca minimamente invasiva (CCMI) é alternativa consagrada para tratamento das cardiopatias congênitas. Curto período de internação, baixos custos e retorno precoce às atividades habituais estão bem documentados.

Objetivo: Demonstrar a experiência de nosso serviço com cirurgia cardíaca minimamente invasiva em cardiopatia congênita do adulto.

Métodos: No período de março de 2007 a novembro de 2016, 60 adultos portadores de cardiopatia congênita (CC), 36 (60\%) do

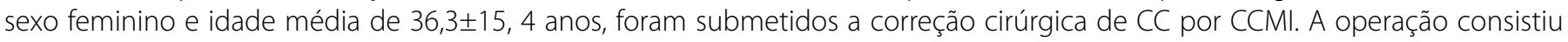
em minitoracotomia no $4^{\circ}$ espaço intercostal direito ou esquerdo de 3 a $5 \mathrm{~cm}$, através de acesso inframamário ou perimamilar. Utilizaram-se circulação extracorpórea (CEC) periférica e clampeio transtorácico. Foram tratados 39 casos de comunicação interatrial; destes, 4 de tipo seio venoso, 6 casos de comunicação interventricular perimembranosa, 3 casos de estenose aórtica congênita, 5 casos de fístula coronário-pulmonar, 2 casos de persistência do canal arterial e 5 casos de membrana subaórtica.

Resultados: A correção primária foi possível em todos os casos. Ecocardiograma transesofágico transoperatório confirmou a

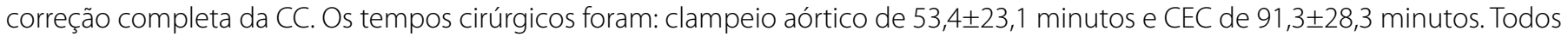
os pacientes foram extubados ainda na sala cirúrgica, permaneceram 1,5 $\pm 0,7$ dias na UTI e receberam alta hospitalar com média de 2,8 1 1,1 dias de pós-operatório, sem intercorrências ou óbitos. Um paciente foi a óbito 3 anos após o procedimento e uma paciente foi reoperada. Após período médio de 5 anos, 98,3\% dos pacientes sobreviveram.

Conclusão: A correção cirúrgica de CC em adultos por meio de CCMI é possível e cursa com excelentes resultados, como curta permanência hospitalar e ótimo efeito estético. A técnica minimamente invasiva é segura e rotina em nosso serviço.

\section{TL $06 \bullet 14 \mathrm{~h} 15-14 \mathrm{~h} 30$}

\section{Abordagem híbrida em um único estágio de pacientes portadores de tetralogia de Fallot e atresia pulmonar com comunicação interventricular e colaterais sistêmico-pulmonares}

Leonardo Augusto Miana, Daniel Peres Guimarães, Maria Raquel Massoti, Luiz Fernando Caneo, Guilherme Viotto, Carla Tanamati, Juliano Gomes Penha, Aida L. Turquetto, Elisio Rebouças, Germana Coimbra, José Honório Palma, Filomena Regina B. G. Gallas, Raul Arrieta, Marcelo Biscegli Jatene

Introdução: A sala híbrida de cirurgia tornou-se uma realidade nas cardiopatias congênitas. Na tetralogia de Fallot (T4F) e atresia pulmonar com comunicação interventricular (AP+CIV), muitas vezes são necessários vários procedimentos cirúrgicos e por cateter até estabelecer a conexão do ventrículo direito (VD) com as artérias pulmonares (APs).

Objetivo: Avaliar a experiência inicial da abordagem em sala híbrida, em um único estágio, de pacientes com diagnóstico de T4F e AP+CIV portadores de colaterais sistêmico-pulmonares (CSP).

Métodos: Estudo retrospectivo de 9 pacientes operados no período de 1 ano. Idade mediana de 10 meses) e peso de $7 \mathrm{~kg}$. Procedimentos em sala cirúrgica híbrida com realização de embolização de CSP pré-circulação extracorpórea (CEC) e/ou angiografia pós-CEC, com ou sem implante de stents no pós-operatório.

Resultados: Em 7 pacientes foi realizada a embolização de CSP com sucesso no período pré-CEC (77,8\%). Todos os pacientes foram submetidos a implante de tubo VD-APS. Em 2 casos (22,2\%) associou-se unifocalização de CSP às APS centrais. O tempo mediano de CEC foi de 165 minutos e de anóxia de 81 minutos. Angiografia pulmonar na saída de CEC foi realizada em 5 casos (55,5\%), com necessidade de implante de stent nas APS em 3 deles (66,6\%). Dois pacientes evoluíram para óbito em 30 dias (22,2\%).

Conclusão: A embolização de CSP pré-CEC e a realização de angiografia na saída de CEC em sala cirúrgica híbrida é factível e permite encurtar o tempo cirúrgico, evitar parada circulatória total, avaliar o resultado cirúrgico no intraoperatório e corrigir eventuais lesões residuais. 


\section{APRESENTAÇÃO DE TEMAS LIVRES - Pediatria}

TL $07 \bullet 14 \mathrm{~h} 30-14 \mathrm{~h} 45$

\section{Análise de efetividade a curto e médio prazo da neovalva aórtica na operação de Ross em pacientes pediátricos}

Camila Roginski Guetter, Cilomar Martins de Oliveira Filho, Cristiano Gustavo Hahn, Fabio Binhara Navarro, Gustavo Klug Pimentel, Gustavo Tedeschi dos Santos, Vinicius Woitowicz, Alexandre Gadelha dos Santos, Francisco Diniz Affonso da Costa, Nelson Itiro Miyague, Leonardo Andrade Mulinari

Introdução: Tem-se mostrado que a melhor indicação para a cirurgia de Ross é a população pediátrica, principalmente em razã do crescimento somático desses pacientes.

Objetivo: Avaliar resultados a curto e médio prazo do autoenxerto pulmonar em via de saída do ventrículo esquerdo na operação de Ross em pacientes pediátricos.

Métodos: Foram revisados retrospectivamente pacientes pediátricos submetidos à cirurgia de Ross entre 2005 e 2016. Todos os pacientes foram incluídos no estudo, excluindo-se neonatos. Avaliaram-se características demográicas e operatórias, funcionamento do autoenxerto, reoperações, mortalidade precoce e tardia.

Resultados: Trinta e cinco pacientes foram avaliados. A idade na cirurgia foi de 9,65 $\pm 4,59$ anos, sendo 21 pacientes (60\%) do sexo masculino. A indicação cirúrgica consistiu em dupla lesão em 21 pacientes (60\%), insuficiência em 6 (17,14\%) e estenose em 6 $(17,14 \%)$, e 3 (8,57\%) portavam valva aórtica bicúspide. A técnica utilizada foi substituição total da raiz aórtica em todos os casos, exceto um. Homoenxerto foi utilizado para a reconstrução da neovalva pulmonar. A técnica de Konno foi associada em 8 pacientes, bem como correção de comunicação interventricular em 2 e plastia mitral em 1 paciente. O tempo de seguimento médio foi $5,90 \pm 2,68$ anos. Não houve dilatação do autoenxerto ou necessidade de reoperação. Não houve mortalidade precoce ou tardia. Dois pacientes evoluíram tardiamente com disfunção valvar importante, sendo um deles com estenose e outro com dupla lesão severa, ambos sem indicação de reintervenção no momento.

Conclusão: A operação de Ross é uma excelente alternativa cirúrgica para tratamento de valvopatias aórticas na população pediátrica, mantendo bom desempenho valvar a curto e médio prazo, sem mortalidade.

\section{TL $08 \bullet 4 h 45-15 h$}

\section{Fatores de risco para troca valvar pulmonar em pacientes no pós-operatório tardio de tetralogia de Fallot}

Maria Raquel Massoti, Luiz Fernando Caneo, Aida Turquetto, Juliano Gomes Penha, Carla Tanamati, Leonardo Augusto Miana, Marcelo Biscegli Jatene

Introdução: Apesar de a insuficiência pulmonar ser tolerada no pós-operatório de tetralogia de Fallot (ToF), muitos pacientes necessitam ser reoperados para troca da valva pulmonar (TVP). A sobrecarga volumétrica imposta ao ventrículo direito pode resultar em dilatação e disfunção pregressiva.

Objetivo: Avaliar pacientes submetidos a troca tardia da valva pulmonar após correção de tetralogia de Fallot, bem como fatores de risco para mortalidade e tempo de internação hospitalar.

Métodos: Avaliação retrospectiva de 86 pacientes submetidos a TVP entre julho de 2010 e dezembro de 2015, dos quais foram analisados parâmetros perioperatórios como tempo de circulação extracorpórea (CEC), volumes ventriculares, fração de ejeção, tempo de internação, mortalidade, entre outros. As análises descritivas e de regressão logística foram realizadas utilizando SPSS19.

Resultados: O volume diastólico final do ventrículo direito (VDFVDI) médio pré-operatório foi de 151,3 mL/m2 e a fração de ejeção do ventrículo direito (FEVD) foi de 40,21\%. Oitenta e um pacientes foram submetidos ao implante de bioprótese e 5 de conduto de homoenxerto. Ocorreram 3 óbitos hospitalares. Não houve correlação entre VDFVDI e FEVD com a mortalidade hospitalar. Maiores VDFVDI, tempo de pinçamento da aorta e circulação extracorpórea apresentaram maior tempo de estadia na unidade de terapia intensiva. Com tempo de seguimento médio de 2,5 anos, 70\% dos pacientes estavam em classe l e 30\%, em classe II.

Conclusão: Não houve correlação entre VDFVDI, FEVD, FEVE e mortalidade hospitalar. Observamos menor tempo de internação na UTI nos pacientes com menor tempo de CEC e naqueles em que os procedimentos foram realizados sem pinçamento aórtico. A troca da valva pulmonar pode ser realizada com baixa mortalidade, mesmo na presença de grandes volumes do ventrículo direito. 



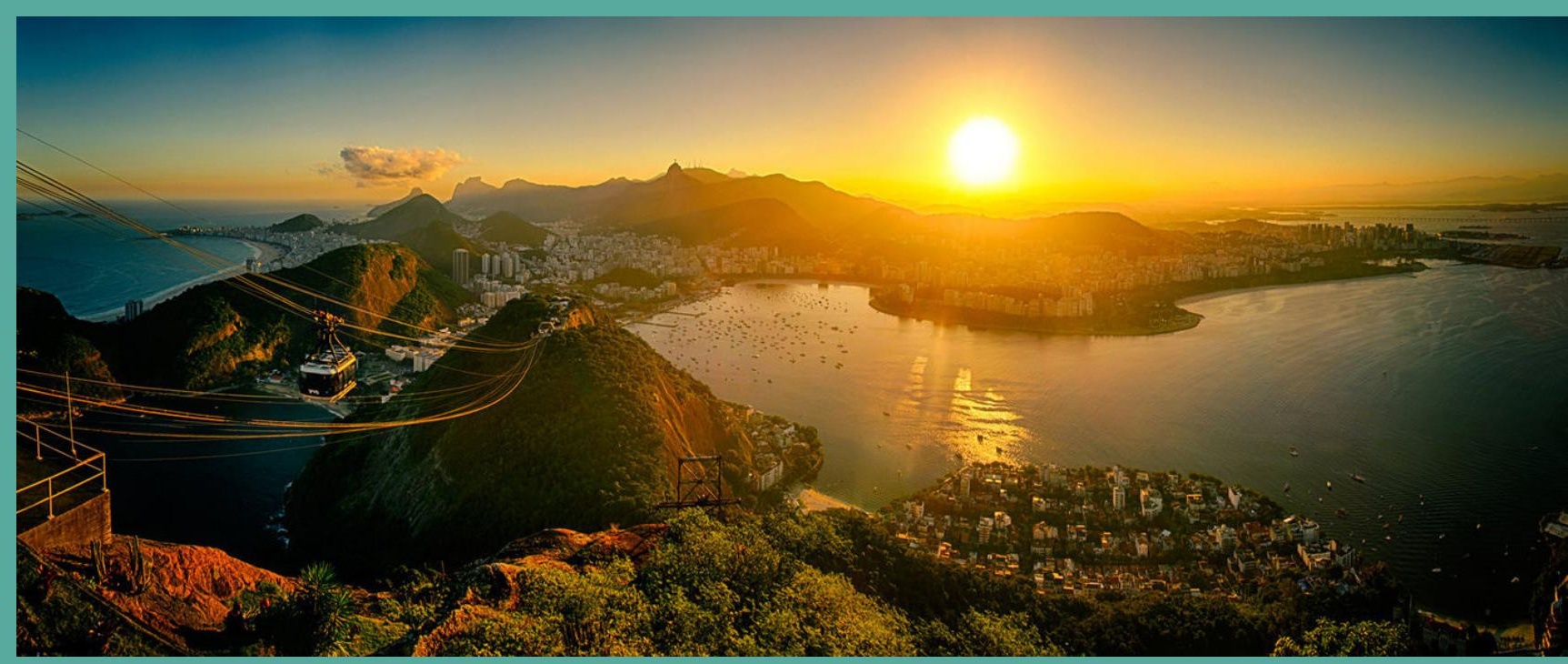

\section{Temas Livies}

Fisioterapia 


\section{TL $01 \cdot 8 \mathrm{~h} 30-8 \mathrm{~h} 40$}

\section{O produto da distância caminhada pelo peso corporal versus distância percorrida na avaliação da cinética do consumo de oxigênio em pacientes coronariopatas}

Isadora Salvador Rocco, Hayanne O. Pauletti, Marcela Viceconte, Bruna C. Matos-Garcia, Natasha O. Marcondi, Rita Simone L. Moreira, Ross Arena, Walter J. Gomes, Solange Guizilini

Introdução: Recentes estudos propuseram o produto da distância caminhada pelo peso corporal (DxP) como um novo método para acessar a capacidade funcional pelo teste de caminhada de 6 minutos (TC6M), inicialmente proposto para pacientes com doença pulmonar obstrutiva crônica.

Objetivo: Comparar o DxP versus distância percorrida no TC6M durante a avaliação da cinética do consumo de oxigênio (VO2) em coronariopatas.

Métodos: Foram avaliados 41 coronariopatas (22 com fração de ejeção de ventrículo esquerdo [FEVE] preservada e 19 com FEVE reduzida). A cinética do VO2 foi obtida durante o TC6M acoplado a analisador de gases portátil e a resposta foi modelada usando análise de regressão não linear monoexponencial. O tempo de resposta médio (wMRT) foi corrigido pela diferença do VO2 em estado estável e em repouso. O DxP foi calculado pelo produto da distância $(\mathrm{km})$ pelo peso corporal $(\mathrm{kg})$.

Resultados: A média da distância percorrida foi $441 \pm 67 \mathrm{~m}$ e do DxP foi $30,9 \pm 6,7 \mathrm{~km}$. kg-1. O DxP foi fortemente correlacionado ao VO2ss e a WMRT; já a distância se correlacionou apenas moderadamente com essas variáveis. Houve correlação positiva forte entre FEVE e DxP $(r=0,72 ; P<0,0001)$ e apenas positiva moderada entre FEVE e distância $(r=0,55 ; P=0,0011)$. O DxP foi superior à distância em identificar a diferença entre grupos, estratificados por FEVE, demonstrado por tamanho de efeito de $d=1,30$ versus $d=0,82$, respectivamente.

Conclusão: O DxP mostrou-se superior à distância percorrida em acessar a capacidade funcional submáxima obtida pela cinética do consumo de oxigênio. O DxP parece mimetizar o trabalho durante a caminhada, sugerindo ser melhor parâmetro para avaliar pacientes coronariopatas, especialmente na ausência da avaliação das variáveis cardiopulmonares.

\section{TL $02 \bullet 8 h 40-8 h 50$}

\section{Novas perspectivas para avaliação da resistência muscular respiratória baseado no limiar de carga pressórica: comparação entre dois protocolos de obtenção do PthMax}

Guilherme de Souza Areias, Luan Rodrigues Santiago da Silva, Michel Silva Reis

Introdução: Os protocolos de avaliação de resistência muscular inspiratória têm sido aplicados com limitada precisão para estratificação da real carga de fadiga do indivíduo. São necessários novos protocolos que permitam medidas mais fidedignas.

Objetivo: Avaliar, por meio de um estudo prospectivo, transversal, cego e aleatorizado, a utilidade do protocolo do tipo rampa para mensuração do limiar de carga pressórico sustentado durante um teste incremental (PthMax).

Métodos: Os indivíduos foram submetidos a três avaliações: i) pressão inspiratória máxima (PImáx); ii) protocolo de degrau, que estratifica o indivíduo em plataformas percentuais de 10\%, variando de 60 a 90\% da PImáx; iii) o protocolo de rampa, que consiste em aumentos progressivos de 2\% da PImáx, variando de $3 \mathrm{cmH} 2 \mathrm{O}$ (carga mínima) até 100\% (PImáx). Análise estatística: teste t-pareado e correlação de Pearson com $\mathrm{P}<0,05$. Bland-Altman para concordância intermétodos.

Resultados: Noventa e dois indivíduos foram submetidos aos protocolos de degrau e rampa e separados as variáveis masculinas e femininas, respectivamente: i) percentual de fadiga $(68,04 \pm 9,10$ vs. 66,53 $\pm 8,30)$ e $(67,86 \pm 13,29$ vs. 64,73 $\pm 17,81)$, não apresentando

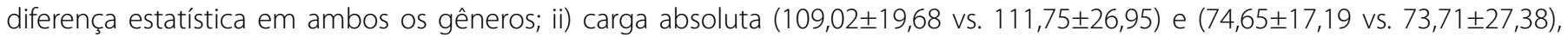
também sem apresentar diferença estatística em ambos os gêneros; iii) número de incursões apresentou diferença estatística $(23,51 \pm 13,66$ vs. $34,70 \pm 6,76)$ e $(23,73 \pm 14,14$ vs. $33,18 \pm 8,89)$. Os testes de Pearson demonstraram forte correlação na variável Carga Absoluta (Pthmáx) nos protocolos, sendo $\mathrm{P}=0,78$ para homens e $\mathrm{P}=0,82$ para mulheres.

Conclusão: O teste incremental do tipo rampa se mostrou tão útil para avaliar a RMI quanto o teste incremental em degraus. 


\title{
APRESENTAÇÃO DE TEMAS LIVRES - Fisioterapia
}

\section{TL $03 \bullet 8$ 8h50-9h}

Similaridade entre os valores de consumo de oxigênio obtidos pela fórmula preditiva e medidos pelo teste de exercício cardiopulmonar na potência do limiar de anaerobiose e no pico do exercício em homens jovens, adultos e portadores de insuficiência cardíaca

\author{
Rafael Santiago Floriano, Alexandre Fenley, Michel Silva Reis
}

\begin{abstract}
Introdução: O teste de exercício cardiopulmonar (TECP) é o padrão-ouro não invasivo de avaliação global da integridade dos ajustes fisiológicos no organismo humano durante a execução do exercício físico. Alternativamente, a avaliação da capacidade funcional e aeróbia tem sido executada por meio de fórmulas de predição com correção para as características antropométricas e carga de trabalho executada. No entanto, esse método de avaliação pode ter diferenças significativas, quando utilizado em uma população de idosos aparentemente saudáveis e em idosos portadores de insuficiência cardíaca (IC) crônica.
\end{abstract}

Objetivo: Avaliar se há similaridade entre os valores de VO2 em homens jovens e idosos aparentemente saudáveis e portadores de IC, obtidos pela fórmula de predição e os medidos pelo TECP na potência do limiar de anaerobiose (LA) e no pico do exercício.

Métodos: Cinquenta e quatro homens foram submetidos ao TECP em cicloergômetro para determinação do LA. Posteriormente, foi realizada a estimativa da carga da potência do LA por meio de fórmula de predição. Os valores do VO2 obtido e da carga estimada foram comparados.

Resultados: Embora não tenha sido observada diferença estatística entre o consumo de oxigênio na potência do LA e no pico do exercício, obtidos pela fórmula preditiva quando comparado ao TECP nos jovens; os idosos saudáveis e os portadores de IC mostraram valores superestimados.

Conclusão: A fórmula preditiva aplicada se revelou adequada para os indivíduos jovens. No entanto, superestimou os idosos e os pacientes com IC crônica.

\section{TL $04 \bullet 9 h-9 h 10$}

\section{Percentual de queda da distância no teste de caminhada de 6 minutos como marcador prognóstico após cirurgia de revascularização do miocárdio}

Hayanne Osiro Pauletti, Isadora S. Rocco, Marcela Viceconte, Bruna C. Matos-Garcia, Natasha O. Marcondi, Rita Simone L. Moreira, Nelson A. Hosse Jr, Ross Arena, Walter J. Gomes, Solange Guizilini

Introdução: O prejuízo na capacidade funcional no pós-operatório (PO) precoce de cirurgia de revascularização do miocárdio (CRM) é inevitável. Porém, uma queda na distância percorrida no teste de caminhada de 6 minutos (DTC6M) entre o pré e o pósoperatório que seja clinicamente relevante não está bem estabelecida.

Objetivo: Investigar o percentual de queda na DTC6M no PO precoce e sua habilidade como marcador prognóstico de resultados clínicos a médio prazo no PO de CRM.

Métodos: Estudo de coorte prospectivo. Foram avaliados 54 pacientes submetidos à CRM eletiva. A queda na DTC6M foi obtida pela diferença percentual do TC6M no pré-operatório e no quinto dia de PO (POD5). A força muscular respiratória foi obtida no préoperatório e no POD5. Seguimento de 3 meses após CRM foi realizado para avaliar desfechos clínicos.

Resultados: Houve queda significativa na DTC6M no POD5 em relação ao pré $(33,1 \pm 16,5 \%, P<0,0001)$. Correção negativa moderada foi observada entre a porcentagem de queda na DTC6M com a pressão inspiratória máxima $(r=-0,66, P<0,001)$ e com a pressão expiratória máxima $(r=-0,61, P<0,001)$ no POD5. Houve correlação positiva moderada entre porcentagem de queda na DTC6M e tempo de ventilação mecânica $(r=0,60, P<0,01)$. Os preditores independentes da porcentagem de queda na DTC6M foram fração de ejeção de ventrículo esquerdo, uso de circulação extracorpórea e tempo de internação na unidade de terapia intensiva. Análise ROC revelou que o melhor valor de corte da porcentagem de queda na DTC6M para predizer resultados clínicos em 3 meses foi 34,6\% (AUC: 0,82; sensibilidade: 78,95\%; especificidade: 76.19\%; $P=0,0001$ ).

Conclusão: Queda percentual da DTC6M maior que 34,6\% no PO precoce em relação ao pré-operatório pode ser considerada um marcador prognóstico de resultados clínicos a médio prazo após CRM. 


\title{
TL 05 • 9h10-9h20
}

\section{Limitação ao fluxo expiratório em pacientes com doença arterial coronariana durante exercício de moderada e alta intensidade}

Viviane Castello Simões, Marlus Karsten, Vinicius Minatel, Rodrigo Polaquini Simões, Ester Silva, Nayara Yamada Tamburús, Ross Arena, Audrey Borghi-Silva, Aparecida Maria Catai

\begin{abstract}
Introdução: Estudo prévio realizado em nosso laboratório mostrou que há limitação ao fluxo expiratório (LFE) em pacientes com infarto do miocárdio (IM) durante a moderada e alta intensidade do exercício aeróbio; porém, não foi objetivo desse estudo avaliar a LFE na doença arterial coronariana (DAC) estável.
\end{abstract}

Objetivo: Testar a hipótese de que o IM é responsável pela LFE no exercício de moderada intensidade e que, pacientes com DAC estável (sem IM) somente apresentem LFE no exercício de alta intensidade.

Métodos: Quarenta e um homens (40-65 anos) foram alocados em quatro grupos: 1) DAC estável (GDAC) ( $n=9)$, 2) IM recente (GIMR) ( $n=8), 3)$ IM tardio (GIMT) $(n=12)$ e, 4) grupo controle saudável (GC) $(n=12)$. Dois testes de exercício cardiopulmonar em carga constante foram realizados em ordem crescente de intensidade; nos últimos três minutos de cada teste foram obtidas alças fluxo-volume corrente durante o exercício, sendo essas posicionadas dentro da máxima alça fluxo-volume obtida na situação de repouso prévia ao exercício, possibilitando assim avaliar a presença de LFE.

Resultados: LFE não esteve presente em nenhum sujeito do GC. Entretanto, LFE ocorreu em 3 dos 9 sujeitos do GDAC, 4 dos 8 sujeitos do GIMR e 7 dos 12 sujeitos do GIMT, mas somente o GIMR e o GIMT apresentaram diferença significativa em relação ao GC. No exercício de alta intensidade todos os grupos estudados apresentaram LFE e nenhuma diferença significativa foi observada entre eles: 7 dos 12 sujeitos do GC, 8 dos 9 sujeitos do GDAC, 7 dos 8 sujeitos do GIMR e 11 dos 12 sujeitos do GIMT. Quando as intensidades foram comparadas entre si, o GC e o GDAC apresentaram maior número significativo de indivíduos com LFE na alta intensidade em relação à moderada intensidade do exercício. Em relação ao grau de LFE na moderada intensidade do exercício, somente o GIMR e o GIMT apresentaram valores significativamente maiores em relação ao GC. Similarmente, durante a alta intensidade do exercício, esses maiores valores do grau de LFE também foram observados, porém somente no GIMT comparado ao GC. Quando ambas as intensidades foram comparadas, todos os grupos estudados apresentaram um aumento significativo do grau de LFE na alta intensidade em relação à moderada intensidade do exercício.

Conclusão: LFE esteve presente nos dois grupos com IM em ambas intensidade do exercício; já o GDAC e o grupo saudável apresentaram LFE somente na alta intensidade do exercício. Desta forma, a presença de LFE observada na moderada intensidade do exercício nos grupos com IM pode estar relacionada às consequências da isquemia e não à DAC.

\section{TL $06 \bullet 9 h 20-9 h 30$}

\section{Valor adicional do limiar anaeróbio em um modelo de predição de morte geral em uma coorte urbana de pacientes com cardiopatia chagásica}

Roberto Ribeiro da Silva, Emilia Matos do Nascimento, Basílio de Bragança Pereira, Michel Silva Reis, Roberto Coury Pedrosa

Introdução: O limiar anaeróbico (LA) é reconhecido como medida objetiva que reflete variações no metabolismo dos músculos esqueléticos no exercício. Seu valor prognóstico nas cardiopatias não chagásicas está bem estabelecido. Entretanto, a avaliação de risco de morte em cardiopatas chagásicos está relativamente estabelecida pelo escore de Rassi. Porém, o valor adicional que o LA pode trazer ao escore ainda não foi estudado.

Objetivo: Avaliar se o LA apresenta efeito adicional ao escore de Rassi em cardiopatas chagásicos.

Métodos: Estudo prospectivo de coorte dinâmica com análise retrospectiva de prontuários, sendo analisados 150 prontuários de pacientes. Foram selecionados para a coorte 45 prontuários de pacientes que realizaram teste cardiopulmonar de exercício (TCPE) entre 1996 e 1997, sendo acompanhados até setembro de 2015. Análise dos dados, para detectar associação entre variáveis estudadas, foi realizada por meio de um modelo de regressão logística (Curvas ROC). Foi utilizado software R versão 2.13.

Resultados: Oito pacientes (17,78\%) morreram até setembro de 2015, sendo 7 (87,5\% dos óbitos) por causas cardiovasculares, dos quais 4 (57,14\%) eram de alto risco pelo escore. Com escore de Rassi como variável independente, sendo óbito o desfecho, obtivemos área sob a curva $(A \cup C)=0,711$, com R2=0,214. Com $L A$ como variável independente, verificamos $A U C=0,706$, com $R 2=0,078$. Definindo escore de Rassi mais o LA como variáveis independentes, foi obtida $A \cup C=0,800$ e $R 2=0,263$. Instituindo como variáveis independentes o escore de Rassi, LA e a interação entre essas variáveis, achamos AUC=0,797 e R2=0,267.

Conclusão: Quando a variável LA é incluída na regressão logística, ela aumenta em $5 \%$ a explicação $\left(R^{2}\right)$ à estimativa de morte. 


\section{APRESENTAÇÃO DE TEMAS LIVRES - Fisioterapia}

\section{TL $07 \bullet 14 h-14 h 10$}

\section{Comparação da influência da ventilação mecânica não invasiva na perfusão tecidual após cirurgia de revascularização do miocárdio com versus sem CEC}

Natasha de Oliveira Marcondi, Isadora Salvador Rocco, Hayanne Oshiro Pauletti, Isis Begot Valente, Thatiana Cristina Alves Peixoto, Natalia Ribeiro dos Anjos, Rita Simone Lopes Moreira, Walter José Gomes, Solange Guizilini

Introdução: Disfunção orgânica e hipóxia tecidual são complicações frequentes no pós-operatório de cirurgia de revascularização do miocárdio. A ventilação mecânica não invasiva (VMNI) tem mostrado um papel importante na melhora da contratilidade cardíaca pelos efeitos da pressão positiva.

Objetivo: Comparar a influência da ventilação mecânica não invasiva na perfusão tecidual nos pacientes submetidos a cirurgia de revascularização do miocárdio com versus sem circulação extracorpórea (CEC).

Métodos: Foram incluídos 100 pacientes no pós-operatório de cirurgia de revascularização do miocárdio eletiva e divididos em dois grupos: com e sem CEC e foram submetidos à VMNI após extubação. Os pacientes foram submetidos a quatro coletas de sangue arterial e venoso, na admissão, após extubação, durante e após a VMNI, para mensuração de lactato arterial e saturação venosa central de oxigênio (ScVO2).

Resultados: Em ambos os grupos, a ScVO2 diminui após a extubação comparada à admissão. Os pacientes com CEC apresentaram maior aumento da ScVO2 durante e após 20 minutos de VMNI comparados ao grupo sem CEC $(P<0,05)$. O lactato arterial se mostrou significantemente mais alto nos pacientes com CEC em todos os momentos. Durante e após 20 minutos da VMNI, ambos grupos apresentaram queda significativa do lactato, entretanto, o grupo com CEC apresentou maior queda $(P<0,002)$.

Conclusão: A VMNI determinou melhora aguda na perfusão tecidual nos pacientes submetidos à cirurgia de revascularização do miocárdio, especialmente no grupo com CEC.

\section{TL $08 \bullet 14 h 10-14 h 20$}

\section{Ventilação oscilatória no exercício físico é capaz de refletir gravidade de pacientes com insuficiência cardíaca crônica}

Hugo Valverde Reis, Priscila Sperandio, Adriano Barroco, Clynton Lourenço Correa, José Alberto Neder, Audrey Borghi-Silva, Michel Silva Reis

Introdução: A insuficiência cardíaca (IC) crônica é caracterizada pela clássica disfunção ventricular esquerda com o surgimento da disfunção muscular, impactando na qualidade de vida do paciente. Alguns parâmetros podem refletir gravidade na IC crônica, e muitos deles são obtidos pelo teste de esforço cardiopulmonar (TECP), assim como a presença da oscilação ventilatória durante o exercício físico (OVE).

Objetivo: Caracterizar a presença da oscilação ventilatória e correlacioná-la com outras variáveis funcionais.

Métodos: Quarenta e seis homens com IC crônica foram recrutados para a realização do TECP máximo e correlacionou-se a resposta destas variáveis com outras variáveis clínicas.

Resultados: A OVE foi observada em 16 dos 24 pacientes que realizaram o TECP, assim como o VENCO2 slope > 34 e VO2 pico $<14 \mathrm{~mL} / \mathrm{kg} / \mathrm{min}$ em 14 e 10 pacientes, respectivamente. Não houve diferença nas variáveis clínicas e do TECP dos pacientes que apresentaram OVE quando comparados aos que não apresentaram. Não houve diferença nas variáveis do TECP e variáveis clínicas quando comparados os pacientes que apresentaram OVE junto a valores de VENCO2 slope $>34$ aos pacientes que apresentaram somente uma dessas respostas.

Conclusão: Este estudo mostrou uma incidência de pacientes com OVE e valores baixos de VO2 pico e valores elevados de VE/ VCO2 slope, mas eles não mostraram diferença nas outras variáveis com valor prognóstico, assim como nenhuma influência da presença da OVE nos outros parâmetros do TECP nessa população, sugerindo que essa variável do TECP possa ser um marcador independente de gravidade nos pacientes com IC crônica. 


\section{TL $09 \cdot 14 \mathrm{~h} 20-14 \mathrm{~h} 30$}

\section{Comparação da força muscular respiratória no pré e pós-operatório de cirurgia cardiopediátrica}

Larissa de Holanda Lessa, Evelin Aparecida Batista de Oliveira, Glauber Schettino da Silva, Jéssyca Lane Fausto Lira, Juliana dos Santos Oliveira, Jussara Guimarães da Rocha Lima, Karolyne Soares Barbosa Granja, Lara dos Santos Camilo, Clara Regina Batista Hora, Lumara Pecllysya Santos Lima, Marília Gameleira Bonfim Padilha, Michelle Santa Rita Palmeira, Tânia Mayla Rezende de Gusmão, Adriana Santos Cunha Calado, Ana Carolina do Nascimento Calles, José Wanderley Neto, Ricardo César Cavalcanti

Introdução: A força muscular respiratória é avaliada a partir da pressão respiratória máxima que é gerada após inspiração (PImáx) e expiração (PEmáx) completas. Como consequência, os principais sintomas apresentados pelas crianças portadoras de cardiopatias congênitas são dispneia e fadiga.

Objetivo: Comparar a força muscular respiratória no pré e pós-operatório de cirurgia cardiopediátrica.

Métodos: Trata-se de um estudo de corte transversal e prospectivo. As crianças foram avaliadas no pré-operatório e no segundo e terceiro dias de pós-operatório. Para determinar a PImáx e a PEmáx das crianças foi realizada manovacumetria, seguindo o protocolo de Black e Hyatt. Os valores foram expressos em cmH2O e determinados por meio de um manovacuômetro analógico. Para analisar os valores preditos, foram adotadas as equações de Neder. A análise estatística descritiva foi realizada com média, desvio padrão e porcentagem e a análise analítica com teste ANOVA, considerando $\mathrm{P}<0,05$.

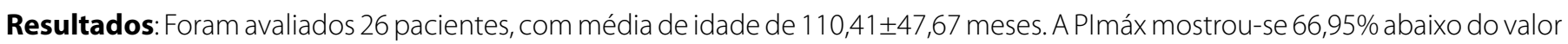
predito, houve uma queda significativa entre o pré-operatório $(-76,92 \pm 39,37 \mathrm{cmH} 2 \mathrm{O})$ e o $2^{\circ} \mathrm{PO}(-72,30 \pm 35,36 \mathrm{cmH} 2 \mathrm{O})(\mathrm{P}<0,001)$ e um ganho significativo de força muscular inspiratória no $3^{\circ} \mathrm{PO}(-80,38 \pm 34,58 \mathrm{cmH} 2 \mathrm{O})(\mathrm{P}<0,001)$. Enquanto a PEmáx mostrou-se $69,98 \%$ abaixo do valor predito, no pré-operatório $(56,04 \pm 16,87 \mathrm{cmH} 2 \mathrm{O}), 2^{\circ} \mathrm{PO}(56,04 \pm 23,17 \mathrm{cmH} 2 \mathrm{O})$ e $3^{\circ} \mathrm{PO}(56,66 \pm 34,25 \mathrm{cmH} 2 \mathrm{O})$ não apresentaram diferença significativa $(P=0,995)$.

Conclusão: Todas as médias de PImáx e PEmáx alcançadas estão abaixo dos valores de referência normais.

\section{TL $10 \cdot 14 \mathrm{~h} 30-14 \mathrm{~h} 40$}

\section{Comparação da força muscular respiratória e periférica no pré e pós-operatório de cirurgia cardíaca}

Karolyne Soares Barbosa Granja, Thayse Campos de Menezes, Juliana Emanuelle Santos Luz Barros, Larissa de Holanda Lessa, Bruna Rodrigues Moraes, Evelin Aparecida Batista de Oliveira, Glauber Schettino da Silva, Jéssyca Lane Fausto Lira, Lumara Pecllysya Santos Lima, Marília Gameleira Bonfim Padilha, Michelle Santa Rita Palmeira, Jussara Guimarães da Rocha Lima, Lara dos Santos Camilo, Tânia Mayla Rezende de Gusmão, Ana Carolina do Nascimento Calles, José Wanderley Neto, Ricardo César Cavalcanti

Introdução: Grande parte dos pacientes submetidos a cirurgia cardíaca (CC) apresentam episódio de debilidade muscular no préoperatório, o qual se acentua após o procedimento cirúrgico.

Objetivo: Avaliar pacientes submetidos a CC com comparação da força muscular respiratória, força muscular periférica e dor no pré e pós-operatório.

Métodos: Estudo transversal e prospectivo. Foi realizada a mensuração da força muscular respiratória (FMR) por meio da manovacuometria, da força muscular periférica pelo do escore do Medical Research Council (MRC) e da dor avaliada pela escala visual analógica (EVA), no pré, $3^{\circ}$ e $6^{\circ}$ dias de pós-operatório (PO). A análise estatística foi feita por meio das variáveis contínuas, apresentadas como média e desvio padrão. A normalidade foi testada por meio do teste de Lilliefors. A comparação entre média foi realizada por meio da ANOVA.

Resultados: Foram avaliados 46 pacientes, com idade média de 60,5+9,2 anos, com predomínio do sexo masculino (63\%). A pressão inspiratória máxima (PImáx) apresentou queda de 21,1\% em relação ao $3^{\circ} \mathrm{PO}(\mathrm{P}<0,01)$ e um retorno aos valores iniciais no $6^{\circ} \mathrm{PO}$, enquanto a pressão expiratória máxima (PEmáx) no pré-operatório se mostrou 18\% abaixo do previsto, além de um decréscimo de 29,4\% entre o pré-operatório e o 30 PO ( $P<0,01)$. Verificou-se, em todos os tempos avaliados, que houve predomínio de dor leve.

Conclusão: Os achados deste estudo confirmam a correlação entre FMR e FMP, e confirmam que a redução dos valores da FMR e da FMP no $\mathrm{PO}$ não se reverte completamente aos níveis pré-operatórios até o $6^{\circ}$ dia de $\mathrm{PO}$. 


\section{APRESENTAÇÃO DE TEMAS LIVRES - Fisioterapia}

\section{TL $11 \bullet 14 \mathrm{~h} 40-14 \mathrm{~h} 50$}

\section{Associação entre a força muscular periférica e respiratória com a performance no teste Timed Up and Go em idosos internados em enfermarias de cirurgia vascular}

Mauricio de Sant' Anna Junior, Jéssica Nunes Ribeiro, Mariana Alexandre dos Santos, Christiano Barbosa, Juliana Verdini de Carvalho Pinheiro, Ana Clara Felix Xavier, Mariana Fonseca Vaz, Thais Vasconcelos de Mello, Leonardo Fonseca, Ana Paula Novelo, Vitor Savino, Daniele Muniz, Luciana Moisés Camilo, Ricardo Gaudio

Introdução: A hospitalização é considerada fator de risco para perda da função muscular periférica e respiratória. OTimed Up and Go (TUG) foi proposto para avaliar equilíbrio e risco de queda em condições de transferência.

Objetivo: Correlacionar a força muscular periférica e respiratória com a performance no TUG em idosos internados na enfermaria de cirurgia vascular (ECV) de dois hospitais do município do Rio de Janeiro.

Métodos: Estudo transversal realizado nas ECV do Hospital Federal dos Servidores do Estado (HFSE) e Hospital Universitário Pedro Ernesto (HUPE). Foram excluídos pacientes com: instabilidade hemodinâmica; aneurisma de aorta; delirium; dispneia classe funcional IV; drenos; infusão venosa; dor (EVA $\geq 8$ pontos; doença osteomuscular limitante; trombose venosa profunda; déficit cognitivo e amputações recentes. Foram mensuradas: pressão inspiratória (PImáx) e expiratória (PEmáx) máximas e força de preensão manual (FPM). OTUG foi realizado utilizando cadeira padronizada e corredor demarcado. As variáveis foram expressas como média \pm desvio padrão, para comparação entre valores previstos e obtidos utilizou-se o teste t e para correlação foi utilizado o teste de Pearson; para ambos foi adotada a significância $\mathrm{P}<0,05$.

Resultados: Foram recrutados 72 idosos; 22 foram excluídos. Permaneceram 50 idosos (63\% H; 37\% M). Quanto a PIMáx, PEmáx e FPM, foram observadas diferenças significativas. Houve associação entre a FPP e a PEmáx com o TUG e não foi observada correlação com a PImáx.

Conclusão: Houve associação entre a força muscular periférica e respiratória com a performance no TUG para os idosos internados na ECV do HFSE e do HUPE.

\section{TL $12 \cdot 14 \mathrm{~h} 50-15 \mathrm{~h}$}

\section{Modulação autonômica da frequência cardíaca de pacientes com doença arterial coronariana inseridos em um programa de reabilitação cardiovascular}

Glauco César da Conceição Canella, Márcia Maria Faganello, Jéssica Guimarães Al-Lage, Robison José Quitério

Introdução: A doença arterial coronariana (DAC) é mais comumente devida à obstrução das artérias coronárias por uma placa ateromatosa, e as alterações no sistema nervoso autonômico estão relacionadas ao grau de oclusão da artéria coronária, resultando em um aumento da modulação simpática.

Objetivo: Investigar a modulação simpática e parassimpática da frequência cardíaca em pacientes com doença arterial coronariana inseridos em um programa de reabilitação.

Métodos: CEP: 1.779.423/2016, a amostra foi composta por 23 pacientes, sendo 6 mulheres e 17 homens, com idade de 59,3 $\pm 10,4$ anos, acometidos com DAC. Foram registrados os intervalos RR (iRR) em repouso durante 5 minutos, através de um sistema de eletrocardiografia (WinCardio, Micromed, Brasil), selecionando 256 pontos mais estáveis e calculados os índices de variabilidade da frequência cardíaca (VFC) (Kubios 2.2). Para análise da modulação autonômica foram calculados os índices no domínio do tempo representativos da modulação parassimpática (RMSSD; pNN50 e HF), simpática (SDNN e LF) e plot de Poincaré. Foram adotados os valores normativos entre o $5^{\circ}$ e o $95^{\circ}$ percentil, propostos por Samitto e Böckelmann (2016).

Resultados: Amostra: 23 pacientes, idade 59 10,4 anos, IMC: $27,9 \pm 4$ kg/m2. Índices da variabilidade da frequência cardíaca em pacientes com DAC e valores normativos propostos por Samitto e Böckelmann (2016).

Conclusão: Diante disto, podemos concluir que não houve alteração na modulação simpática e parassimpática da frequência cardíaca, confirmando a preservação desse ramo neural nos pacientes acometidos com doença arterial coronariana. 



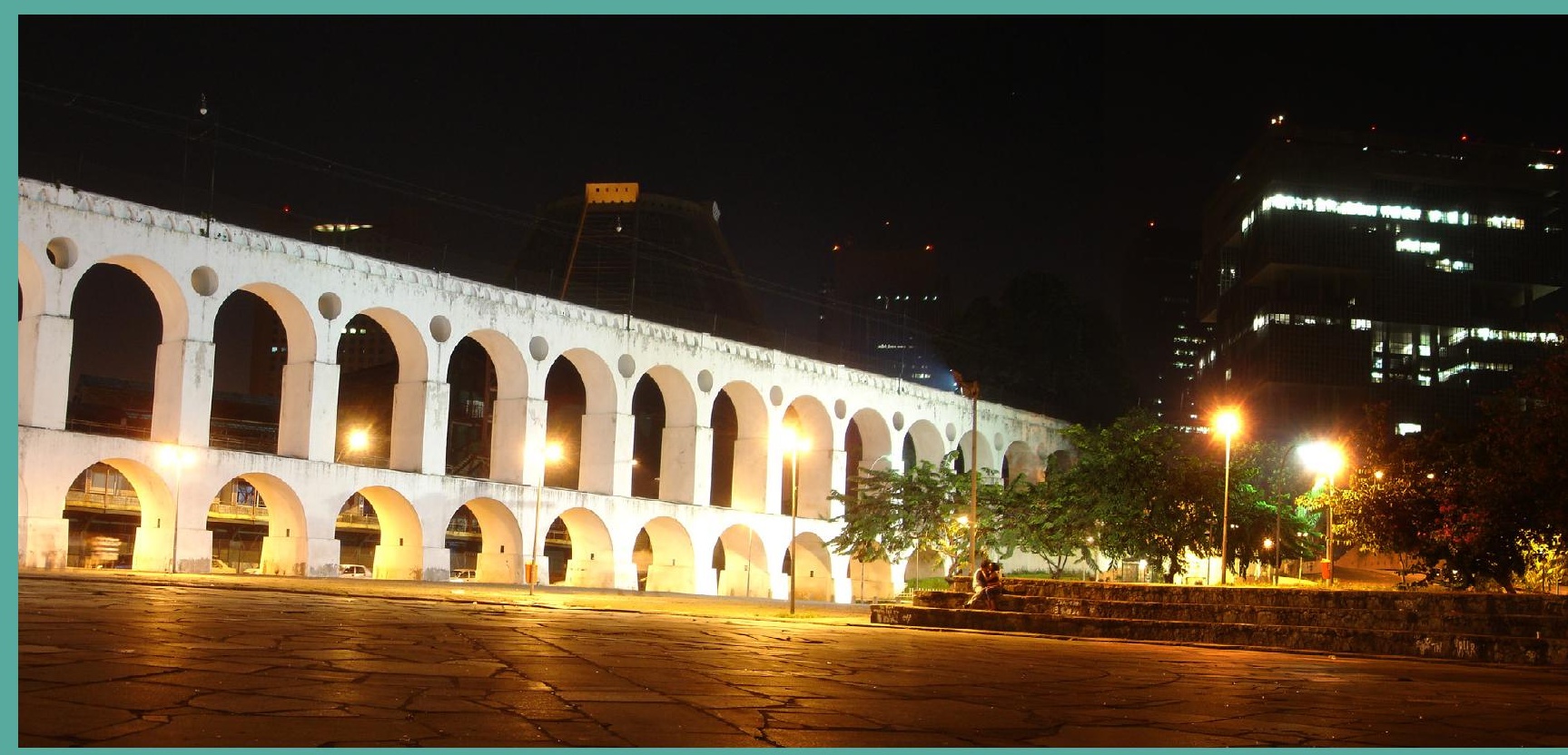

\section{Pôsteres}

Fisioterapia 


\section{PO 01}

\section{Pacientes com cirrose hepática apresentam menor capacidade cardiopulmonar quando comparados com indivíduos saudáveis}

Igor Nasser Cardoso, Ingrid Dias, Anderson Dias, Renata Perez, Luciana Malosá, Humberto Miranda, Michel Silva Reis

Introdução: Cirrose hepática é o estágio final de agressões crônicas, resultando em uma distorção parenquimal e disfunção hepática. A doença pode levar os pacientes a uma debilidade, resultando em um hábito de vida sedentário e diminuindo a capacidade funcional desses indivíduos.

Objetivo: Avaliar e comparar a capacidade cardiopulmonar de portadores de cirrose hepática com indivíduos saudáveis.

Métodos: Foram selecionados 20 indivíduos sedentários, entre 50 e 70 anos, sendo 10 portadores de cirrose e 10 saudáveis. Os participantes foram submetidos a um teste cardiopulmonar incremental no cicloergômetro (acréscimo de 10 watts por minuto). Como critério para interrupção do teste, foram consideradas respostas inadequadas da pressão arterial, alteração eletrocardiográfica

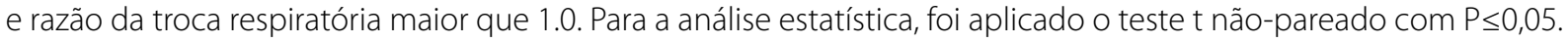

Resultados: Os valores de troca respiratória em repouso foram mais elevados nos pacientes com cirrose quando comparado ao grupo controle, indicando um estresse nesses indivíduos, mesmo sem intervenção do exercício. Os valores de VENO2 e VENCO2 foram menores nos cirróticos no limiar anaeróbico, assim como no pico do exercício, mostrando uma capacidade cardiopulmonar reduzida. Além disso, os hepatopatas tiveram uma resistência ao teste, uma vez que o tempo de tolerância foi menor que os saudáveis.

Conclusão: Os pacientes cirróticos apresentam menor tolerância a fadiga e baixa capacidade cardiopulmonar comparado com indivíduos saudáveis.

\section{PO 02}

\section{Repercussões ventilatórias durante o deslocamento cardíaco para a realização durante cirurgia de revascularização do miocárdio com técnica aorta no-touch. Um estudo piloto}

Isadora Salvador Rocco, Solange Guizilini, Alexandre R. Carvalho, Gustavo M. Murai, Isis Begot, Nelson A. Hossne Jr, Eduardo G. Chamlian, Walter J. Gomes

Introdução: A cirurgia de revascularização do miocárdio com técnica aorta no-touch (NT-OPCAB) tem sido recomendada para o tratamento de pacientes com alto risco perioperatório de lesão neurológica. No entanto, o deslocamento do coração para obter exposição adequada para a construção dos enxertos pode induzir alterações hemodinâmicas. Entretanto, as alterações ventilatórias ainda requerem investigação.

Objetivo: Avaliar as alterações sequenciais ventilatórias durante o deslocamento do coração em pacientes submetidos a NTOPCAB.

Métodos: Coorte prospectivo envolvendo 27 pacientes submetidos a NT-OPCAB. A perfusão tecidual foi avaliada continuamente pela saturação venosa central de oxigênio (ScvO2) através do sistema FloTrac/PreSep/VigileoT (Edwards Lifesciences). Os pacientes foram ventilados em PCV para garantir inicialmente $6 \mathrm{ml} / \mathrm{Kg}$ peso predito, PEEP=5, FiO2 para $\mathrm{SaO} 2>90 \%$. Como desfecho foram analisados alterações de volumes, FiO2 e saturação arterial de oxigênio durante os deslocamentos do coração. Os parâmetros foram avaliados 5 min antes, durante e 5 min após cada anastomose (descendente anterior, descendente posterior, marginal e diagonal). Concentrações de lactato arterial foram obtidas no pós-operatório imediato (POI).

Resultados: O número de anastomoses variou de 2 a 4 (média=2,7) por paciente. Não houve alterações significantes do volume, $\mathrm{FiO} 2$ e saturação arterial de oxigênio durante os deslocamentos do coração nas diferentes anastomoses $(P>0,05)$. Além disso, nenhuma associação foi observada entre os parâmetros ventilatórios e concentrações de lactato arterial no POI.

Conclusão: $O$ deslocamento cardíaco para a realização de NT-OPCAB não influenciou os paramentos ventilatórios. As concentrações de lactato no POI parecem estar relacionadas a outras alterações que não as variações ventilatórias. Assim, a NT-OPCAB não cursa com repercussões ventilatórias que possam alterar perfusão tecidual. 


\title{
EXPOSIÇÃO DE PÔSTERES \\ - Fisioterapia
}

\section{PO 03}

\section{Comparação entre dois instrumentos de avaliação da pressão inspiratória máxima}

\author{
Luan Rodrigues Santiago da Silva, Guilherme de Souza Areias, Michel Silva Reis
}

Introdução: Desde que surgiu a avaliação da força muscular inspiratória pela pressão inspiratória máxima (PIMáx), várias manobras e dispositivos foram utilizados para tentar estratificar seus valores de forma cada vez mais fisiológica e precisa.

Objetivo: Validar por meio de um estudo prospectivo, transversal, cego e aleatorizado, a semelhança de valores de PIMáx obtidos pelo dispositivo Power Breathe K5 em comparação ao manovacuômetro digital MVD 300.

Métodos: Foram triados indivíduos jovens e saudáveis que foram submetidos a duas avaliações de PIMáx, em ambos os aparelhos de forma aleatorizada. A PIMax foi determinada com a execução da manobra de Miller (volume residual até capacidade pulmonar total) para ambos dispositivos. Foi realizado um período de familiarização com os dispositivos e a manobra de forma a minimizar vieses de aprendizado. Análise Estatística: Teste t-pareado para comparação dos valores de PIMáx nos aparelhos (valores média e dp). Foi realizado também, o teste de comparação de Pearson, com $\mathrm{P}<0,05$. Aplicada analise de concordância de Bland-Altman.

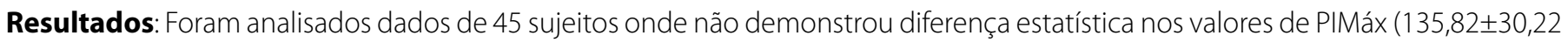
vs. 127,71 29,59$)$. Dados evidenciados pela análise de Pearson demonstram correlação forte e significativa para os valores absolutos de PIMàx em ambos os dispositivos com r=0,73. A análise da concordância demonstra que os valores de PIMáx mostraram uma distribuição simétrica ao redor da linha média.

Conclusão: O aparelho Power Breathe apresenta valores de PIMáx similares aos valores obtidos pelo Manovacuometro Digital, portanto, pode ser utilizado para aferição da força muscular inspiratória.

\section{PO 04}

\section{Validade de fórmulas preditivas da capacidade funcional em atletas de futebol feminino}

\section{Alexandre Fenley, Rafael Floriano, Michel Silva Reis}

Introdução: O teste cardiopulmonar (TCP) é o padrão ouro para avaliação da capacidade funcional. No entanto, a sua aplicabilidade é limitada por se tratar de um teste de alto custo. A literatura nos apresenta diversas fórmulas para predição de consumo de oxigênio (VO2) para avaliação funcional.

Objetivo: Comparar uma fórmula de predição de VO2 e do equivalente metabólico (MET) com o VO2 e o MET obtidos no TCP durante o limiar de anaerobiose e no pico do exercício.

Métodos: Foram avaliadas 18 jogadoras de futebol profissional (idade $26,07 \pm 4,35$ ) do time de futebol feminino da Marinha do Brasil. As voluntárias recrutadas passaram pelas seguintes etapas: a) Avaliação clínica; e, b) TCP máximo ou sintoma-limitado. OTCP foi executado em esteira ergométrica por meio do protocolo de Bruce. O VO2 ((0.2 x velocidade) + (0.9 x velocidade x inclinação) + 3.5 - velocidade em mph e inclinação \%.) e equivalente metabólico (MET = (6x FCindex) -5), foram estimados a partir de variáveis individuais de cada voluntária e comparadas com o VO2 e MET obtidos no limiar de anaerobiose (LA) e no pico do TCP. Para análise estatística foram aplicados testes de normalidade, homogeneidade e teste t pareado onde $\mathrm{P}<0,05$.

Resultados: O VO2 estimado pela fórmula no pico do exercício e no LA não mostrou diferença significativa. No entanto, o equivalente metabólico estimado se revelou subestimado ao MET obtido $(\mathrm{P}<0,05)$.

Conclusão: A fórmula do VO2 predita foi adequada para avaliar a capacidade funcional da população estudada. No entanto a fórmula do MET subestimou as atletas. 
SESSÃO I

21 de abril • sexta-feira
SALA DE PÔSTERES

10h - $10 \mathrm{~h} 45$
EXPOSIÇÃO DE PÔSTERES

- Fisioterapia

\section{PO 05}

\section{Comparação da força muscular periférica no pré e pós operatório de cirurgia cardíaca pediátrica}

Joyce Annenberg Araújo dos Santos, Evelin Aparecida Batista De Oliveira, Glauber Schettino da Silva, Jéssyca Lane Fausto Lira, Juliana dos Santos Oliveira, Jussara Guimarães da Rocha Lima, Karolyne Soares Barbosa Granja, Karolyne Soares Barbosa Granja, Clara Regina Batista Hora, Lumara Pecllysya Santos Lima, Marilia Gameleira Bonfim Padilha, Michelle Santa Rita Palmeira, Tânia Mayla Rezende de Gusmão, Adriana Santos Cunha Calado, Ana Carolina do Nascimento Calles, José Wanderley Neto, Ricardo César Cavalcanti

Introdução: A literatura parece ser escassa sobre a condição de força muscular periférica em crianças submetidas a cirurgia cardíaca, porém, sabe-se que a condição patologia tende a apresentar disfunções musculoesquelética. Até o presente momento desconhecemos estudos que tenham avaliado força muscular periférica nessa população.

Objetivo: Comparar a força muscular periférica no pré e pós operatório de cirurgia cardíaca pediátrica.

Métodos: Trata-se de um estudo de corte transversal e prospectivo, realizado em um Hospital de Maceió- Alagoas. As crianças foram avaliadas em três momentos pré operatório, segundo dia de pós operatório e terceiro dia de pós operatório. Para avaliar a força muscular periférica foi utilizado a pontuação do escore do Conselho de Pesquisas Médicas - Medical Research Council - MRC. Por meio desse escore, foi graduado a força em valores compreendidos entre 0 (paralisia muscular total) e 5 (força muscular normal) pela realização voluntária de seis movimentos específicos bilaterais. A pontuação total pode variava de 0 (tetraparesia completa) a 60 (força muscular normal).

Resultados: Foram avaliados 37 pacientes, sendo 62,16\% do sexo masculino e 37,83\% do sexo feminino, com média de idade $86,45 \pm 49,26$ meses. A média dos valores avaliados classificam as crianças com força muscular normal. Porém, a média da força apresenta um decréscimo no $2^{\circ} \mathrm{DPO}(\mathrm{MRC}=57,67 \pm 4)$ quando comparada ao Pré $\mathrm{OP}(\mathrm{MRC}=59,97 \pm 4,89)(\mathrm{P}=0,05)$, tendo um acréscimo no $30 \mathrm{DPO}(\mathrm{MRC}=58,21 \pm 3,80)(\mathrm{P}=0,05)$.

Conclusão: Nosso estudo sugere que crianças submetidas à cirurgia cardíaca, não apresentem déficit de força muscular periférica

\section{PO 06}

\section{Efeitos da EPAP na variabilidade da frequência cardíaca de jovens aparentemente saudáveis}

Clarear Figueiredo Telles, Leonardo da Costa Silva, Michel Silva Reis

Introdução: A literatura é incipiente sobre a resposta da modulação da frequência cardíaca durante a aplicação de diferentes níveis de pressão positiva.

Objetivo: Identificar o comportamento da variabilidade da frequência cardíaca durante a aplicação da EPAP.

Métodos: 12 voluntários saudáveis permaneceram sentados e foram submetidos a coleta dos dados de FC batimento a batimento em três situações: 10 minutos ventilando espontaneamente, 10 minutos ventilando espontaneamente com EPAP de $5 \mathrm{cmH2O}, 10$ minutos ventilando espontaneamente com EPAP de $16 \mathrm{cmH} 2 \mathrm{O}$.

Resultados: Houve um aumento estatisticamente significante nos valores da BF u.n. e redução não progressiva de AF u.n. nas situações sem EPAP, EPAP de 5 e de $16 \mathrm{cmH2O}$. Houve também diferença estatística na média da FC com a aplicação da EPAP $[73,66 \pm 11,71 \times 76,59 \pm 10,99 \times 78,78 \pm 11,86]$, nas situações sem EPAP, EPAP de 5 e de $16 \mathrm{cmH} 2 \mathrm{O}$, respectivamente. Ocorreu um aumento da relação $\mathrm{BF} / \mathrm{AF}$, o que sugere que houve aumento do poder espectral em BF devido à redução da $\mathrm{FR}$ com o uso da

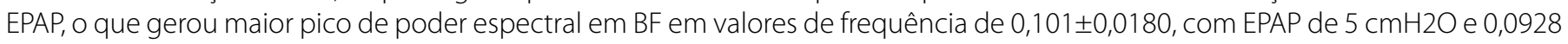
$\pm 0,0210$, com EPAP de $16 \mathrm{cmH2O}$, resultando em valores de AF u.n. menores.

Conclusão: A aplicação da EPAP parece acentuar a arritmia sinusal respiratória tanto em valores de $5 \mathrm{cmH} 2 \mathrm{O}$ quanto em valores de $16 \mathrm{cmH} 2 \mathrm{O}$. 


\section{EXPOSIÇÃO DE PÔSTERES \\ - Fisioterapia}

\section{PO 07}

\section{Avaliação postural de pacientes submetidos a cirurgia cardíaca pediátrica}

Rita de Cássia dos Santos Moreira, Evelin Aparecida Batista De Oliveira, Glauber Schettino da Silva, Jéssyca Lane Fausto Lira, Juliana dos Santos Oliveira, Jussara Guimarães da Rocha Lima, Sarah Carolina Almeida Luna Vieira, Lara dos Santos Camilo, Clara Regina Batista Hora, Lumara Pecllysya Santos Lima, Marilia Gameleira Bonfim Padilha, Michelle Santa Rita Palmeira, Tânia Mayla Rezende de Gusmão, Adriana Santos Cunha Calado, Ana Carolina do Nascimento Calles, José Wanderley Neto, Ricardo César Cavalcanti

Introdução: Tratando-se de cirurgias torácicas, acredita-se que o procedimento deixe uma falha postural como consequência, podendo haver encurtamentos das estruturas anteriores do tórax.

Objetivo: Identificar as alterações posturais de pacientes submetidos a cirurgia cardíaca pediátrica através de um estudo de coorte.

Métodos: Trata-se de um estudo de coorte realizado com crianças submetidas à cirurgia cardíaca para correção de malformações congênitas, sujeitas a uma avaliação postural subjetiva, dada por meio da inspeção visual pelo fisioterapeuta, no pré-operatótio e no $3^{\circ}$ dia de pós-operatório, sendo analisados nas vistas: anterior, perfil esquerdo, perfil direito e posterior. Os dados foram armazenados numa planilha eletrônica. As variáveis do questionário foram tratadas por meio de estatística descritiva, com média, desvio padrão, frequência relativa e absoluta.

Resultados: Participaram do estudo 11 pacientes, com idade média de 6,5 $\pm 3,00$, dos quais 72,7\% apresentou alterações posturais no pós-operatório. Dentre o total de 23 alterações identificadas, 18,2\% dos pacientes tiveram mudança na altura e 45,5\% na rotação interna de ombros, 9,1\% na assimetria de MMSS, 9,1\% no ângulo de Tales, 9,1\% na protrusão e 18,2\% na retração da mandíbula, 27,3\% na protrusão de pescoço, 36,4\% na protrusão de ombro, 9,1\% na retroversão de pelve, 18,2\% apresentou acentuação da cifose torácica e 9,1\% abdome proeminente.

Conclusão: As crianças que são submetidas a cirurgia cardíaca sofrem distúrbios posturais que podem estar associadas ao posicionamento antálgico. Desta forma, torna-se perceptível a importância do fisioterapeuta na reeducação postural destes indivíduos para amenizar complicações as quais estas crianças possam ser expostas ao longo do seu desenvolvimento.

\section{PO 08}

\section{Efeitos da mudança postural sobre a variabilidade da frequência cardíaca de pacientes pós acidente vascular cerebral}

Mariana Nunes Dantas, Marina Bairros Heberle, Elias Tristão, Rafael Zanarino Lobo, Ana Paula Fontana, Michel Silva Reis

Introdução: O comprometimento cerebrovascular dos pacientes pós acidente vascular cerebral (AVC) pode determinar disfunção autonômica. Estratégias para avaliação da modulação autonômica poderiam ser aplicadas para garantir a elaboração de programas de reabilitação mais adequados e seguro. A análise da variabilidade da frequência cardíaca (VFC) na mudança postural tem se mostrado uma conduta eficaz, de baixo custo e não invasiva de avaliação do balanço simpato-vagal.

Objetivo: O objetivo do trabalho foi avaliar a VFC de pacientes pós AVC, durante a mudança postural ativa.

Métodos: Nove pacientes (idade $52.7 \pm 16.5,55 \%$ sexo masculino) foram submetidos a coleta da frequência cardíaca (FC) batimento a batimento por meio do cardiofrequencímetro em repouso nas condições: (i) 10 min na posição supina; e (ii) 10 min na posição sentada. Os dados foram analisados no domínio do tempo pela média da FC e dos intervalos R-R (iR-R) e dos índices rMSSD (raiz quadrada da média das diferenças sucessivas ao quadrado entre os iR-R), SDNN (desvio-padrão dos intervalos R-R), pNN50 (porcentagem dos intervalos RR adjacentes com diferença de duração maior que 50 milissegundos) e NN50 (número de intervalos R-R consecutivos diferentes por mais de 50 milissegundos). Estatística: Teste t-student pareado ou Wilcoxon quando apropriado $(\mathrm{P}<0,05)$. Trabalho aprovado pelo Comitê de Ética em Pesquisa.

Resultados: Não houve resultado significativo, comparando os índices de média da FC, média dos iR-R e índices rMSSD, SDNN, pNN50 e NN50, durante a mudança postural.

Conclusão: Os pacientes estudados não foram capazes de ajustar sua frequência cardíaca conforme a nova demanda imposta através da mudança postural ativa. 
SESSÃO II

21 de abril • sexta-feira
SALA DE PÔSTERES

$16 h-16 h 45$
EXPOSIÇÃO DE PÔSTERES

- Fisioterapia

PO 09

\section{Associação da curva do pulso de oxigênio (PuO2) e a gravidade de pacientes com insuficiência cardíaca crônica}

Aline Virginia Alves, Bárbara Amaral Ferreira, Hugo Valverde Reis, Michel Silva Reis

Introdução: O pulso de oxigênio (PuO2) pode estar reduzido ao exercício físico em pacientes com insuficiência cardíaca crônica (ICC). No entanto, não há literatura solida sobre a associação do comportamento das curvas do PuO2 com a gravidade da doença.

Objetivo: Caracterizar e associar as curvas do PuO2 com a gravidade de pacientes com ICC.

Métodos: Foram incluídos 24 homens com ICC (53.1 13.6 anos, com fração de ejeção do ventrículo esquerdo < 45\%). Os pacientes foram submetidos a avaliação clínica, fisioterapêutica e da função pulmonar. Na sequência, foi executado o TCP em cicloergômetro, com protocolo do tipo rampa (incrementos de $5-10 \mathrm{~W} / \mathrm{min}$ ). O teste foi conduzido até a exaustão máxima ou interrompido por sinais e/ou sintomas de intolerância ao exercício. Por fim, foi determinado o consumo de oxigênio pico, os limiares metabólicos e o comportamento do PuO2. A curva do PuO2 foi classificada de acordo com os tipos de curva (A, B, C e D), onde as curvas progridem do tipo A (comportamento esperado e linear com exercício) para D (curva com descenso, resposta inadequada ao exercício).

Resultados: De acordo com a classificação das curvas, 45,8\% dos pacientes tinham a curva do tipo A, 29,1\% do tipo B e 25\% do tipo C. Adicionalmente, os pacientes com curva tipo C eram os mais gravemente acometidos e com baixa capacidade funcional.

Conclusão: A maioria dos nossos pacientes foram classificados como curva padrão A. No entanto, os pacientes com menor capacidade funcional foram classificados com curva tipo C.

\section{PO 10}

\section{Avaliação do desenvolvimento neuropsicomotor em crianças portadoras de cardiopatia congênita}

Bruna Rodrigues Moraes, Evelin Aparecida Batista De Oliveira, Glauber Schettino da Silva, Jéssyca Lane Fausto Lira, Juliana dos Santos Oliveira, Jussara Guimarães da Rocha Lima, Karolyne Soares Barbosa Granja, Lara dos Santos Camilo, Clara Regina Batista Hora, Lumara Pecllysya Santos Lima, Marília Gameleira Bonfim Padilha, Michelle Santa Rita Palmeira, Tânia Mayla Rezende de Gusmão, Adriana Santos Cunha Calado, Ana Carolina do Nascimento Calles, José Wanderley Neto, Ricardo César Cavalcanti, Leticia de Araújo Costa

Introdução: O desenvolvimento físico e ponderal, além do desenvolvimento motor, cognitivo e neurológico podem ser afetados durante a infância. É considerado desenvolvimento motor a sequência de processos que evoluem com o decorrer da idade cronológica do ser humano, com movimentos simples e desorganizados à execução de habilidades motoras altamente organizadas e complexas.

Objetivo: Avaliar o desenvolvimento neuropsicomotor de crianças com cardiopatias congênitas.

Métodos: Trata-se de estudo transversal realizado em um hospital de Alagoas, no período de Julho a Setembro de 2016. Foram avaliadas as crianças no pré-operatório, no primeiro momento foi extraído do prontuários dados como nome, sexo, idade, tipo de cirurgia, e patologias associadas; para avaliar o desenvolvimento neuropsicomotor foi utilizada a escala de desenvolvimento neuropsicomotor de Denver.

Resultados: Foram avaliadas 13 crianças com média de idade 41,15 13,21 meses, sendo 69,23\% do sexo masculino e 30,76\% do sexo feminino. As crianças apresentavam as seguintes cardiopatias: PCA (30,76\%); CIA (23,07\%); CIV (15,38\%); CIV + CIA (15,38\%); CIA + Correção mitral (7,69\%); CIA + correção de tricúspide (7,69\%). A escala de DENVER foi estratificada em quartil de demonstrando que $38,46 \%$ das crianças encontraram-se no $1^{\circ}$ quartil indicando que as crianças realizam em média 88,67 $\pm 9,14$ das tarefas propostas, $30,76 \%$ estão no $4^{\circ}$ quartil e realizaram em média 11,26 11,38 das tarefas propostas, $15,38 \%$ estão no $2^{\circ}$ quartil e realizam em

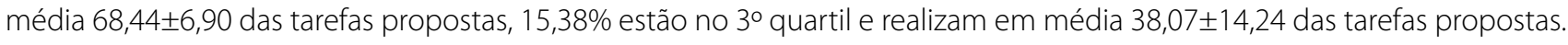

Conclusão: Conclui-se que crianças cardiopatas apresentam atraso em seu desenvolvimento neuropsicomotor 


\section{EXPOSIÇÃO DE PÔSTERES \\ - Fisioterapia}

\section{PO 11}

\section{Incidência de complicações pulmonares em cirurgias cardiopediátricos}

Leticia de Araújo Costa, Evelin Aparecida Batista De Oliveira, Glauber Schettino da Silva, Jéssyca Lane Fausto Lira, Juliana dos Santos Oliveira, Jussara Guimarães da Rocha Lima, Larissa de Holanda Lessa, Lara dos Santos Camilo, Clara Regina Batista Hora, Lumara Pecllysya Santos Lima, Marília Gameleira Bonfim Padilha, Michelle Santa Rita Palmeira, Tânia Mayla Rezende de Gusmão, Adriana Santos Cunha Calado, Ana Carolina do Nascimento Calles, José Wanderley Neto, Ricardo César Cavalcanti. Ricardo César Cavalcanti. Ricardo César Cavalcanti

Introdução: As cardiopatias congênitas são as principais causas de morte em recém-nascidos e afetam 5 a cada 1000 nascidos vivos no Brasil. As crianças que possuem cardiopatias normalmente apresentam alterações da mecânica ventilatória, que juntamente com a cirurgia e o tempo de circulação extracorpórea, aumentam a predisposição para complicações pulmonares.

Objetivo: Avaliar a incidência de complicações pulmonares em crianças submetidas à cirúrgica cardíaca.

Métodos: Tratou-se de um estudo retrospectivo descritivo, feito através da análise dos prontuários de crianças que passaram por cirurgia cardíaca em um hospital de Maceió, sendo separadas de pela idade. Foi realizada média e desvio padrão da idade, tempo de circulação extracorpórea (CEC) e tempo de internação e frequência e porcentagem do diagnostico, sexo e das complicações.

Resultados: Foram avaliadas 13 prontuários com pacientes com idade $\leq 72$ meses, com idade média de 56,3 $\pm 15,78$ meses, tempo

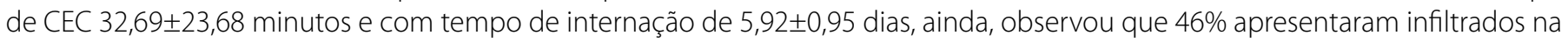
imagem radiológica, 15\% tiveram pneumotórax, 8\% apresentaram congestão pulmonar e 31\% não tiveram complicações. E 10

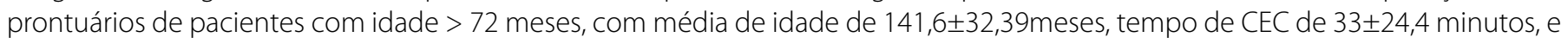
com tempo de internação de 8,2 $\pm 3,52$ dias, ainda, observou que 30\% apresentaram infiltrados, 20\% atelectasias e derrame pleural, $10 \%$ congestão pulmonar e $40 \%$ não apresentaram complicações.

Conclusão: De acordo com os resultados obtidos, foi observado que as complicações mais comuns são: a presença de infiltrados radiológicos e pneumotórax. Ainda, notou-se que a maioria dos pacientes não apresentaram complicações.

\section{PO 12}

\section{Correlação entre tempo de ventilação mecânica invasiva e complicações pulmonares em crianças submetidas à cirurgia cardíaca}

Karolyne Soares Barbosa Granja, Evelin Aparecida Batista De Oliveira, Glauber Schettino da Silva, Jéssyca Lane Fausto Lira, Juliana dos Santos Oliveira, Jussara Guimarães da Rocha Lima, Larissa de Holanda Lessa, Lara dos Santos Camilo, Clara Regina Batista Hora, Lumara Pecllysya Santos Lima, Marília Gameleira Bonfim Padilha, Michelle Santa Rita Palmeira, Rita de Cássia dos Santos Moreira, Adriana Santos Cunha Calado, Ana Carolina do Nascimento Calles, José Wanderley Neto, Ricardo César Cavalcanti

Introdução: O tempo de ventilação mecânica invasiva (VMI) no pós-operatório de cardiopatias congênitas está relacionada a fatores pré-operatórios, intraoperatório e pós-operatório. A demora no desmame da VMI pode trazer complicações pulmonares e atrofia diafragmática decorrente da perda progressiva de fibras musculares.

Objetivo: Correlacionar o tempo de ventilação mecânica invasiva e as complicações pulmonares em crianças que passaram por cirurgia cardíaca.

Métodos: Tratou-se de um estudo retrospectivo descritivo, feito com crianças que passaram por procedimento cirúrgico decorrentes de cardiopatias congênitas, que necessitaram de intubação orotraqueal. Foi feito a análise por meio de média e desvio padrão da idade e do tempo de VMI e frequência e porcentagem do sexo e complicações pulmonares. Ainda foi feita a correlação de Pearson, entre o tempo de ventilação e a presença de complicação pulmonar.

Resultados: Foram analisados 44 prontuários de crianças que passaram por procedimento cirúrgico, sendo 19 (43,18\%) do sexo feminino

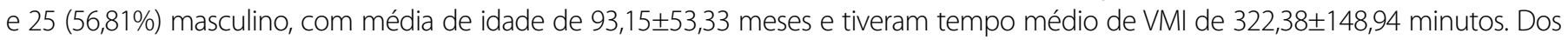


SESSÃO II

21 de abril • sexta-feira
SALA DE PÔSTERES

$16 h-16 h 45$
EXPOSIÇÃO DE PÔSTERES

- Fisioterapia

pacientes que apresentaram complicações 11,36\% tiveram pneumotórax, 20,45\% congestão pulmonar, 9,09\% derrame pleural e 9,09\% atelectasia. Foi observado uma correlação moderada e positiva com valor de r=0,62, com valor de significância de $\mathrm{P}<0,001$.

Conclusão: De acordo com os resultados obtidos, observou-se que as complicações pulmonares mais frequentes são os pneumotórax e as congestões pulmonares e que quanto maior o tempo de ventilação mecânica invasiva maior incidência de complicações pulmonares.

\section{PO 13}

\section{Resposta da modulação autonômica cardíaca frente à fisioterapia cardiovascular no pós-operatório imediato de cirurgia de revascularização do miocárdio: relato de casos}

Bianca Lopes Silva, Hugo Valverde Reis, Ana Carolina Cossio Rodriguez, Priscila de Souza, Isabela de Andrade, Michel Silva Reis

Introdução: A fisioterapia após a cirurgia cardíaca de revascularização do miocárdio (CRM) é um diferencial indispensável para minimizar as disfunções respiratórias e autonômicas.

Objetivo: Avaliar a resposta da modulação autonômica cardíaca frente à fisioterapia cardiovascular no pós-operatório imediato de pacientes submetidos à cirurgia de revascularização do miocárdio.

Métodos: Relato de casos, prospectivo. Foram avaliados cinco pacientes. A variabilidade da frequência cardíaca (VFC) foi coletada 10min em repouso, e durante a realização da manobra de acentuação da arritmia sinusal respiratória (M-ASR).

Resultados: Os principais achados mostram que durante as duas coletas a maioria dos indivíduos aumentou a FC imediatamente após a CRM, apresentando melhora próximo a alta. A VFC total, diminuiu durante todo o período de pós-operatório em 3 voluntários. $\mathrm{O}$ índice RMSSD diminuiu em todos no D01, melhorando no D04, na maioria deles. 75\% apresentaram aumento do índice de BF (un) imediatamente após a CRM com queda do índice de AF (un), próximo a alta, os valores de BF (un) caíram e os de AF (un) aumentam na maioria dos casos. Sobre os valores da razão entre BF/AF, 4 apresentaram aumento do valor da razão no D01, e todos apresentaram queda no D04.

Conclusão: A fisioterapia cardiovascular baseada em exercícios aeróbios foi capaz de favorecer a melhora na VFC. A amplitude da M-ASR também apresentou valores sugestivos de melhora próximo ao D04 na maioria dos voluntários.

\section{PO 14}

\section{Correlação da escala MRC e dinamometria em pacientes com Insuficiência Cardíaca}

Daniel Santana da Silva, Vanessa Marques Ferreira, Adriane Sartori Barbosa, Beatriz de Andrade Tomazini, Cintia Cristina Camargo, Iracema loco Kikushi Umeda

Introdução: A Insuficiência Cardíaca (IC) é definida como a incapacidade do coração em manter o débito cardíaco necessário à demanda metabólica, tendo como uma das consequências a redução da força muscular e da capacidade funcional.

Objetivo: Verificar se a escala MRC e a dinamometria apresentam correlação em pacientes com insuficiência cardíaca.

Métodos: Foram avaliados nas primeiras 24 horas de internação na unidade de terapia intensiva do Instituto Dante Pazzanese de Cardiologia (IDPC) os pacientes com diagnóstico clínico de miocardiopatia chagásica, valvar e isquêmica em ventilação espontânea. Foram excluídos pacientes em choque cardiogênico, com alterações neurocognitivas e musculoesqueléticas que inviabilizassem os testes. Os testes foram feitos no mesmo momento, no leito, com o uso do dinamômetro de mão (hand grip) e a mensuração da escala de força MRC sempre pelo mesmo avaliador.

Resultados: A escala MRC e a dinamometria tiveram correlação e concordância (P-valor 0,000).

Conclusão: Conclui-se no presente estudo que as escalas MRC e Hand Grip têm correlação entre si para pacientes com insuficiência cardíaca. 


\section{EXPOSIÇÃO DE PÔSTERES \\ - Fisioterapia}

\section{PO 15}

\section{Tromboembolismo pulmonar à cavalero e uso da ventilação mecânica não invasiva (VNI) bilevel na prevenção da intubação orotraqueal}

Valéria Papa, Daniela Caetano Costa dos Reis, Marcela Roberta Amaro, Susana Lacerda Terassi, Itana Brondi Crivelenti de Oliveira Barbieri, Franciane Gonçalves, João Paulo Uvera Ferreira, Silas Lucena Lima

Introdução: O tromboembolismo pulmonar(TEP) caracteriza-se pela impactação de material orgânico no leito arterial pulmonar, com mortalidade entre 10 a 15\% nas primeiras horas. A VNI é um dos avanços da ventilação mecânica nas últimas décadas, responsável pela diminuição da necessidade de intubação, mortalidade e custos

Objetivo: Relato de caso

Métodos: Paciente feminino, 57 anos, há 13 dias submetida à cirurgia de remoção de cisto renal, chega com queixa de dispneia, relatando episódio de síncope assistida; constatado Dímeros-D positivo, BNP (BrainNatriureticPeptidium) 196,1 pg/ml e angio tomografia que evidenciou TEP à cavalero, sendo optado por trombectomia por cateter Pronto. Encaminhada à Unidade Coronariana taquicárdica, mantendo dispneia aos mínimos esforços e dependente de oxigenioterapia (cateter nasal $4 \mathrm{l} / \mathrm{min}$ ). No dia seguinte, mantém taquicardia, sat de $\mathrm{O} 2$ 87\%, evoluindo com piora do quadro de dispneia e dependência de oxigenioterapia alto fluxo. Instalado VNI (bilevel) intermitente (pressão inspiratória média=10 cmH20/ pressão expiratória média=6 cmH20/ volume corrente médio=480 mL). Paciente evolui com sinais de Cor Pulmonale ao ecocardiograma.

Resultados: A partir do $8^{\circ}$ dia apresenta melhora da hipoxemia e dispneia, reduzindo a dependência da VNI e a partir do $10^{\circ}$ dia não necessita de VNI ou oxigênioterapia, conseguindo realizar deambulação, seguindo a progressão do protocolo fisioterapêutico. Submetida no 170 dia à trombectomia por cateter aspirex5, recebe alta após 22 dias de internação.

Conclusão: Nesse caso, a VNI foi capaz de prevenir a intubação orotraqueal e auxiliar a melhora da hipoxemia e dispneia desencadeadas pelo TEP, possibilitando a realização do protocolo de fisioterapia, proporcionando um desfecho clínico favorável.

\section{PO 16}

\section{Reabilitação cardíaca na fase I Pós -IAM: artigo de revisão}

Mayara Karla dos Santos Silva Ramos, Daniella Fonseca, Juliana Oliveira, Liliane F. Souza

Introdução: Na prática clínica ainda é comum ser recomendado um período prolongado de repouso no leito pós-IAM, embora já se saiba que o protocolo de reabilitação cardíaca fase I tenha indicação para ser iniciado de 12 à $24 \mathrm{~h}$ após o evento. Nas décadas de 1950-1960 algumas publicações sinalizavam que, uma mobilização intra hospitalar mais precoce, muito incipiente, com a mudança do paciente do leito para a cadeira, proporcionaria a diminuição dos eventos cardiovasculares. Desde então, cresceu a evidência do baixo risco do exercício no aumento da tolerância ao esforço nos doentes cardíacos, particularmente do infarto agudo do miocárdio.

Objetivo: O objetivo deste artigo de revisão é trazer uma atualização quanto aos protocolos utilizados na reabilitação cardíaca durante a fase I pós IAM de acordo com a sua eficácia.

Métodos: Foi realizada uma revisão da literatura nas bases de dados Physiotherapy Evidence Database (PEDro), LiLacs e Pubmed utilizando as seguintes palavras-chave: cardiac rehabilitation, physiotherapy, myocardial infarction, fisioterapia, infarto agudo do miocárdio, perfil epidemiológico.

Resultados: Foram encontrados 161 publicações, apenas 5 foram selecionadas pois preenchiam os critérios de inclusão: ensaios clínicos que abordavam a Reabilitação Cardíaca após IAM, publicados em Inglês e Português, no período de Janeiro de 2011 a Setembro de 2016.

Conclusão: Diante dos estudos avaliados nesta revisão, pode-se concluir que os programas de reabilitação cardíaca iniciados nas primeiras 24 horas pós IAM e mantidos de forma progressiva após a fase hospitalar apresentam um melhor prognóstico no condicionamento cardíaco do paciente. 



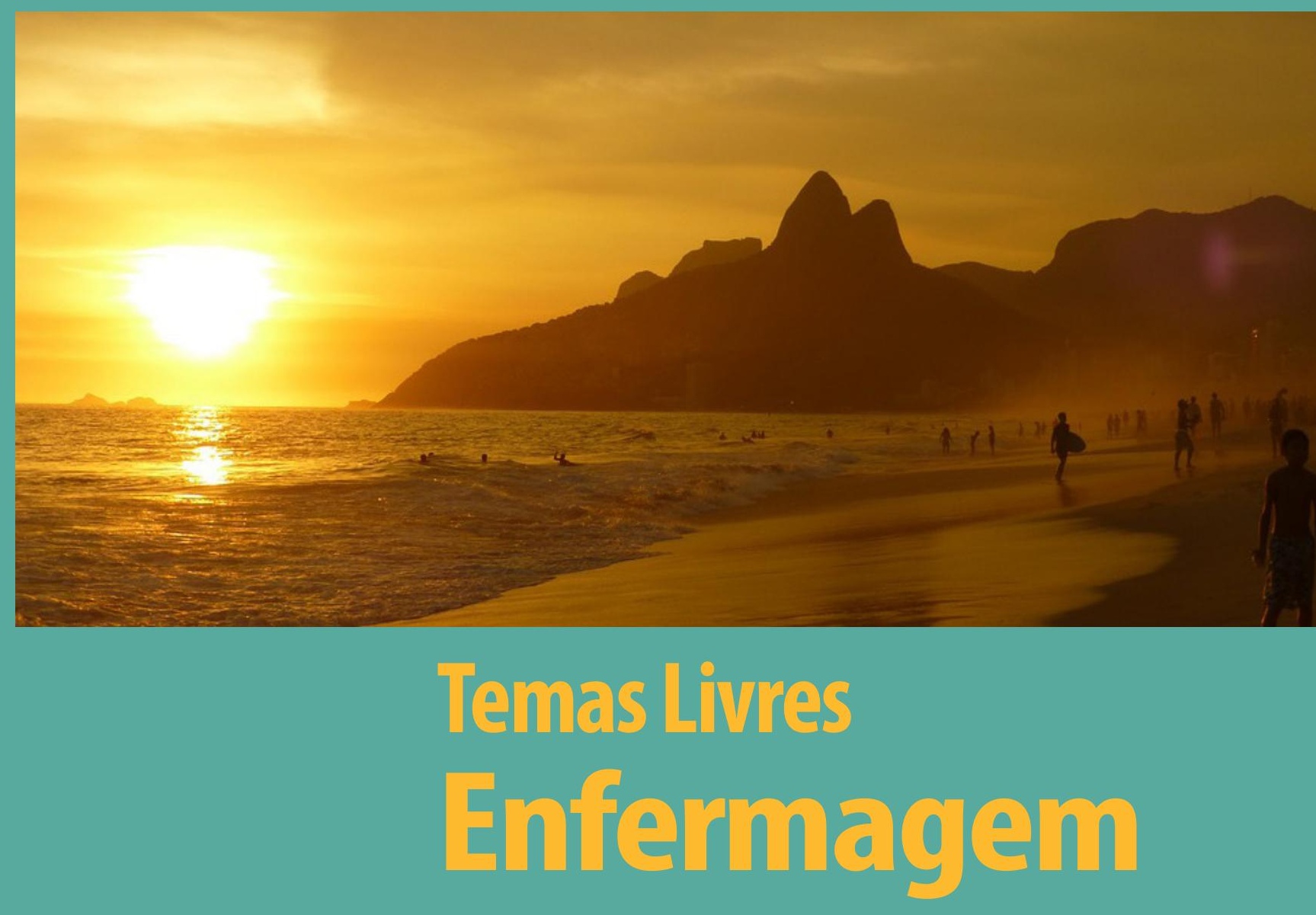




\section{TL 01• 8h15-8h30}

\section{Aplicação de um Escore de Predição de Risco de Mediastinite em Pacientes Submetidos a Cirurgia de Revascularização do Miocárdio Associada ou não à Cirurgia de Troca Valvar}

Bruna Zortea, Letícia Orlandin, Rafael Heiling de Souza, Eneida Rejane Rabelo da Silva, Graziella Badin Aliti

Introdução: A mediastinite é uma complicação da cirurgia cardíaca (CC) que pode ser predita pela avaliação de características préoperatórias em um escore de risco recentemente desenvolvido no Brasil.

Objetivo: Avaliar a categoria de risco do escore como preditor para mediastinite; identificar o perfil dos pacientes com mediastinite e verificar a taxa de mortalidade após 30 dias da cirurgia de revascularização do miocárdio (CRM), associada ou não à troca valvar.

Métodos: Coorte de pacientes submetidos a CC entre 2011 e 2015. Amostra de 620 pacientes, idade $\geq 18$ anos, submetidos à CRM, associada ou não à troca valvar. O escore pontuou reintervenção cirúrgica (3 pontos); doença pulmonar obstrutiva crônica (2); obesidade (2); angina classe IV (1) e politransfusão no pós-operatório (1), classificando em risco baixo (0), médio (1 ou 2), elevado (3 ou 4) e muito elevado (5 ou mais pontos). O desfecho primário foi ocorrência de mediastinite, conforme o Centers for Disease Control and Prevention (CDC).

Resultados: A mediastinite ocorreu em 28 (4,5\%) pacientes. A distribuição do risco na amostra foi baixo, 0 (0\%); médio, 2 (1,1\%); elevado, 8 (25\%) e muito elevado, 18 (64,3\%). A categoria elevado e muito elevado, 26 (43,3\%), foi preditora para ocorrência de mediastinite $(P=0,000)$. Hipertensão $(P=0,032)$, peso $(P=0,000)$, tempo de circulação extracorpórea $(P=0,005)$, tempo de internação $(P=0,000)$, tempo de intubação traqueal $>24$ horas $(P=0,05)$ associaram-se à mediastinite. A taxa de mortalidade geral em 30 dias foi de $7,6 \%$, sendo $1,1 \%$ por mediastinite.

Conclusão: O uso do escore foi capaz de predizer a ocorrência de mediastinite nos pacientes com risco elevado ou muito elevado.

\section{TL $02 \cdot 8 \mathrm{sh} 30-8 \mathrm{~h} 45$}

\section{Fatores de Risco para Tromboembolismo Pulmonar no Pós-Operatório de Cirurgia Cardíaca}

Brenda Gonçalves Donay, Fernanda Lourega Chieza, Marco Aurélio Bizarro dos Santos, Luiz Carlos Bodanese, João Carlos Vieira da Costa Guaragna, João Batista Petracco, Mário Wiehe, Vera Elizabeth Closs, Ellen Hettwer Magedanz

Introdução: Conforme Darze et al., tromboembolismo pulmonar (TEP) é a terceira causa de morte cardiovascular após infarto agudo do miocárdio (IAM) e acidente vascular cerebral (AVC), acometendo cerca de 1/1.000 pessoas/ano.

Objetivo: Identificar fatores de risco para TEP no pós-operatório de cirurgia cardíaca (POCC).

Métodos: Estudo de coorte histórica, coleta realizada de dezembro/2004 a julho/2015, com pacientes de ambos os sexos, submetidos a cirurgia de revascularização miocárdica (CRM), troca valvar (TV) e CRM+TV. Variáveis: idade, sexo, angina instável (Al), fibrilação atrial (FA), obesidade, varizes, fibrinogênio, dislipidemia, doença pulmonar obstrutiva crônica (DPOC), circulação extracorpórea (CEC), ventilação mecânica (VM) e reintubação. Para análise de dados utilizou-se o software SPSS. Para análise descritiva realizaram-se medidas de frequência, tendência e dispersão. Para variáveis categóricas foram realizados testes Quiquadrado e Exato de Fischer, e a comparação entre as medidas contínuas foi realizada pelo teste T de Student. Foi realizada análise multivariada por regressão logística considerando nível de confiança de 95\%.

Resultados: Dos 5.735 pacientes submetidos a cirurgia cardíaca, com média de idade de 59,2 213,0 anos, 63,6\% do sexo masculino, 1,6\% evoluíram com TEP no pós-operatório. Após análise univariada, obteve-se significância para as variáveis CRM, TV, Al, FA, obesidade, varizes, fibrinogênio, dislipidemia, DPOC, $C E C, V M$ e reintubação $(P \leq 0,2)$. Na análise multivariada mostraram-se associadas a TEP: CRM $(P=0,018)$, obesidade $(P=0,003)$, varizes $(P=0,033)$ e reintubação $(P=0,024)$.

Conclusão: É fundamental a avaliação de risco para TEP no POCC, em razão das complicações que este desfecho apresenta. Reforça-se a importância do acompanhamento multiprofissional, particularmente quanto à mobilização precoce, profilaxia e reconhecimento do quadro. 


\section{APRESENTAÇÃO DE TEMAS LIVRES \\ - Enfermagem}

\section{TL $03 \cdot 8 h 45-09 h$}

\section{Dispositivos de Assistência Ventricular Esquerda (LVAD): Custo do Curativo do Sítio de Saída do Driveline}

Ligia Neres Matos, Vanessa Silveira Faria, Tamires Luciana Pena, Ana Luiza Ferreira Sales, Anna Karinina Bitaraes de Sá, Vinicius Assunção P Sobral, Bruno Miranda Marques, Tereza Cristina Felippe Guimarães, Alexandre Siciliano Colafranceschi

Introdução: Infecção no sítio de saída do driveline (DLN) é a causa mais comum de mortalidade durante o suporte com dispositivos de assistência ventricular esquerda (LVAD).

Objetivo: Apresentar o custo do curativo do sítio de saída do DLN.

Métodos: Foram acompanhados seis pacientes que tiveram alta hospitalar após o implante do LVAD no período de março/2012 a março/2016 no Hospital Pró-Cardíaco. Todos os pacientes e cuidadores receberam treinamento das coordenadoras de ventrículo para a realização do curativo do sítio de saída do DLN. O curativo é realizado com técnica asséptica pelo cuidador ou paciente treinado conforme protocolo operacional padrão (POP) para realização deste procedimento.

Resultados: O tempo máximo de follow-up foi de 4 anos e mínimo de 2 meses. Os LVAD utilizados foram: HeartWare (33\%) e HM II (67\%). Com relação à rotina de troca do curativo 16,66\% trocam a cada 7 dias, 33,3\% em dias alternados e 50\% diariamente. No período estudado, o custo médio por curativo foi de $\mathrm{R} \$ 33,77 /$ dia, variando entre $\mathrm{R} \$ 30,66-36,89$. O custo mensal dos pacientes que fazem o curativo em dias alternados foi de $\mathrm{R} \$ 506,62 /$ mês em média. No grupo estudado não houve nenhum caso de infecção de sítio de saída do DLN.

Conclusão: A utilização do protocolo e a padronização do material para a realização do curativo da linha de saída do driveline permitiram estimar o custo do curativo possibilitando uma organização orçamentária pelo paciente, garantindo manutenção e segurança deste procedimento. 



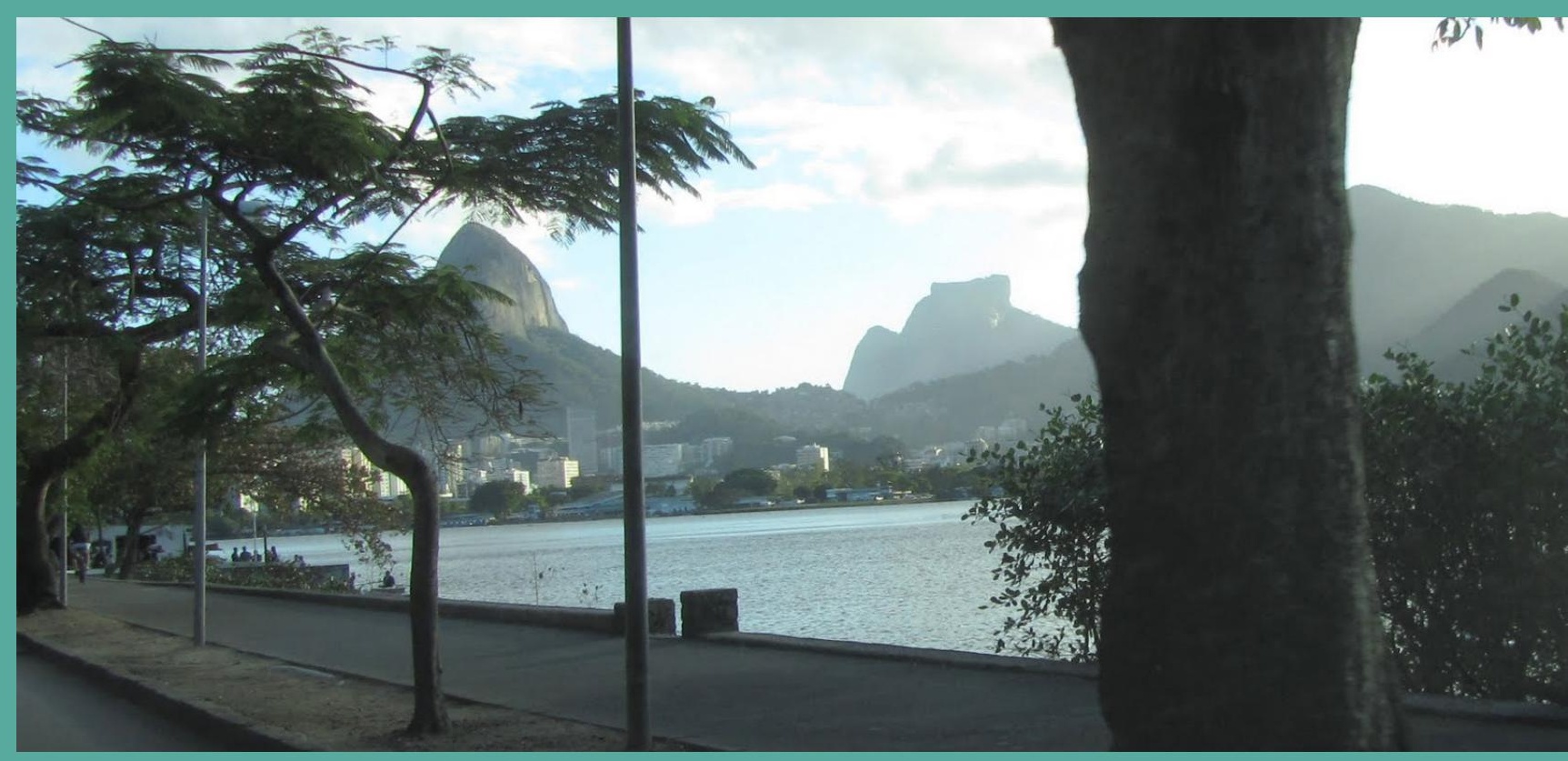

\section{Pôsteres}

Enfermagem 
SESSÃO I

22 de abril • sábado
SALA DE PÔSTERES

10h - $10 \mathrm{~h} 45$
EXPOSIÇÃO DE PÔSTERES

- Enfermagem

\section{PO 01}

\section{Pacientes com cirrose hepática apresentam menor capacidade cardiopulmonar quando comparados com indivíduos saudáveis}

Igor Nasser Cardoso, Ingrid Dias, Anderson Dias, Renata Perez, Luciana Malosá, Humberto Miranda, Michel Silva Reis

Introdução: Cirrose hepática é o estágio final de agressões crônicas, resultando em uma distorção parenquimal e disfunção hepática. A doença pode levar os pacientes a uma debilidade, resultando em um hábito de vida sedentário e diminuindo a capacidade funcional desses indivíduos.

Objetivo: Avaliar e comparar a capacidade cardiopulmonar de portadores de cirrose hepática com indivíduos saudáveis.

Métodos: Foram selecionados 20 indivíduos sedentários, entre 50 e 70 anos, sendo 10 portadores de cirrose e 10 saudáveis. Os participantes foram submetidos a um teste cardiopulmonar incremental no cicloergômetro (acréscimo de 10 watts por minuto). Como critério para interrupção do teste, foram consideradas respostas inadequadas da pressão arterial, alteração eletrocardiográfica

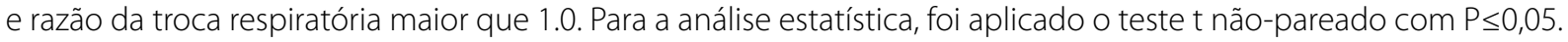

Resultados: Os valores de troca respiratória em repouso foram mais elevados nos pacientes com cirrose quando comparado ao grupo controle, indicando um estresse nesses indivíduos, mesmo sem intervenção do exercício. Os valores de VENO2 e VE/VCO2 foram menores nos cirróticos no limiar anaeróbico, assim como no pico do exercício, mostrando uma capacidade cardiopulmonar reduzida. Além disso, os hepatopatas tiveram uma resistência ao teste, uma vez que o tempo de tolerância foi menor que os saudáveis.

Conclusão: Os pacientes cirróticos apresentam menor tolerância a fadiga e baixa capacidade cardiopulmonar comparado com indivíduos saudáveis.

\section{PO 02}

Melhoria dos Resultados Através de um Programa Baseado no Relatório do Banco de Dados da Sociedade Americana de Cirurgia Torácica (STS) - Primeira Experiência de um Hospital Brasileiro

Denise Louzada Ramos, Nilza Sandra Lasta, Viviam de Souza Ramirez, Camila Gabrilaitis Cardoso, José Carlos Teixeira Garcia, Mariana Yumi Okada, Pedro Gabriel Melo de Barros e Silva, Valter Furlan

Introdução: O banco de dados do STS é uma importante ferramenta para programas de melhoria da qualidade. A maior evidência é baseada em estudos na América do Norte, uma vez que há poucos estudos em outras regiões.

Objetivo: Avaliar o impacto do programa de melhoria da qualidade com base nos relatórios do STS nos resultados de cirurgia de revascularização do miocárdio (CRM).

Métodos: Foi implementado um programa de educação com base em relatórios do STS em 2012, por uma equipe multidisciplinar, visando à redução no tempo de ventilação mecânica (VM), no tempo de permanência na unidade de terapia intensiva (UTI) e também no número de transfusões inadequadas. A equipe educacional desenvolveu um protocolo institucional e utilizou o banco de dados do STS como referência para fazer intervenções com a equipe cirúrgica e clínica. Todos os pacientes submetidos à cirurgia de revascularização do miocárdio foram incluídos e foi comparado o impacto da intervenção educativa.

Resultados: De janeiro de 2012 a outubro de 2016, 1.372 cirurgias de revascularização do miocárdio foram realizadas. O risco previsto de mortalidade pelo escore STS foi de 1,2\% (2012), 0,96\% (2013), 0,99\% (2014), 0,94\% (2015) e 1,06\% (2016). Houve redução na transfusão comparando 2012 e os anos seguintes após a consolidação do programa. Os tempos de VM e permanência hospitalar no pós-operatório se reduziram em 2013 e a redução foi mantida nos anos seguintes.

Conclusão: Os resultados indicam que o programa de melhoria da qualidade com base nos relatórios do STS pode melhorar os resultados em um hospital privado brasileiro. 


\section{EXPOSIÇÃO DE PÔSTERES \\ - Enfermagem}

\section{PO 03}

\section{Evolução em Pós-Operatório de Cirurgia de Revascularização do Miocárdio Isolada de Acordo com a Idade}

Denise Louzada Ramos, Camila Gabrilaitis Cardoso, Nilza Sandra Lasta, Viviam de Souza Ramirez, Larissa Sayuri Nakai, Mariana Yumi Okada, Douglas Ribeiro, José Carlos Teixeira Garcia, Pedro Gabriel Melo Barros, Valter Furlan

Introdução: Com o aumento da expectativa de vida da população, o número de idosos que necessita de algum tipo de intervenção cardiovascular é crescente.

Objetivo: Avaliar as principais diferenças no perfil dos pacientes e na evolução em pós-operatório de cirurgia de revascularização miocárdica isolada (CRM), de acordo com a faixa etária.

Métodos: Foram coletados dados de CRM no período de janeiro de 2012 a outubro de 2016 em um hospital cardiológico de São Paulo, comparando a evolução pós-operatória de três grupos de pacientes: Grupo A, com idade < 60 anos, utilizado como referência, e comparado com o Grupo B (60 a 74 anos) e o Grupo C ( $\geq 75)$.

Resultados: Dentre as 1.312 CRM realizadas, 43\% foram do Grupo A, 48\% do Grupo B e 9\% do Grupo C. As características dos grupos e evolução em pós-operatório.

Conclusão: A idade avançada é um fator associado ao aumento das taxas de complicações, média de tempo IOT, permanência em UTI e hospitalar. Apesar destes aspectos, a maioria dos idosos evolui satisfatoriamente no pós-operatório, e uma estratificação específica acima dos 75 anos poderá ajudar na abordagem destes pacientes.

\section{PO 04}

\section{Incidência de Lesão Neurológica em Pós-Operatório de Cirurgia Cardíaca com Circulação Extracorpórea: Impacto para Assistência de Enfermagem}

Filipe Rodrigo Maimoni Reis do Carmo, Daniel Gomes de Sousa, Elbanir Rosangela Ferreira de Sousa

Introdução: As complicações neurológicas têm importante incidência no pós-operatório das cirurgias cardíacas, tendo considerável impacto nas taxas de mortalidade e no aumento nos gastos hospitalares. Importante estudo randomizado, multicêntrico realizado por Roach et al., contando com 2.417 pacientes, encontrou incidência de 6,1\%.

Objetivo: Caracterizar a clientela submetida a cirurgia cardíaca com circulação extracorpórea e que tenha desenvolvido lesão neurológica, definir a incidência desta complicação e determinar o grau de assistência de enfermagem prestada a esses pacientes.

Métodos: Estudo do tipo coorte, prospectivo, de natureza quantitativa, no qual foi aplicado um teste de avaliação cognitiva no período pré e pós-operatório, além da coleta de dados no prontuário do paciente. Para atingir o objetivo, lançou-se mão da metodologia de Lygia Paim, a qual pontua a assistência de enfermagem prestada.

Resultados: Dos 22 pacientes compreendidos no estudo, 3 (13,63\%) desenvolveram lesão neurológica menor e nenhum paciente evoluiu para lesão neurológica maior. Dos pacientes que desenvolveram déficit cognitivo, apenas um teve aumento na complexidade da assistência de enfermagem prestada.

Conclusão: A lesão neurológica menor teve uma incidência significativa na população estudada e pode implicar um incremento à assistência de enfermagem prestada. Os resultados ainda não são definitivos; assim, o estudo convida os enfermeiros a discutirem e intensificarem as pesquisas de enfermagem no âmbito das complicações neurológicas associadas às cirurgias cardíacas. 
SESSÃO I

22 de abril • sábado
SALA DE PÔSTERES

10h - $10 \mathrm{~h} 45$
EXPOSIÇÃO DE PÔSTERES

- Enfermagem

\section{PO 05}

\section{Evolução do Procedimento de Implante Transcutâneo da Válvula Aórtica (TAVI) em Laboratório de Hemodinâmica de Hospital Público Universitário}

Roselene Matte, Paola Severo Romero, Rejane Reich, Simone Marques dos Santos, Márcia Flores de Casco, Juliana Kruger, Larissa Gussatschenko Caballero, Eduardo Keller Saadi, Orlando Carlos Wender, Eneida Rabelo da Silva

Introdução: $O$ implante transcutâneo da valva aórtica constitui-se em uma alternativa terapêutica menos invasiva para pacientes com risco elevado para cirurgia convencional, seja pela idade avançada, seja por doenças associadas. A equipe de enfermagem, dentro do cenário multidisciplinar, desempenha um papel importante no planejamento, organização e assistência para a segurança do procedimento.

Objetivo: Descrever os casos de TAVI, tempo de preparação de sala, equipe de enfermagem, tempo de procedimento e realização de lista de verificação (check-list).

Métodos: Estudo transversal conduzido no Laboratório de Hemodinâmica de um hospital público universitário. Foram selecionados pacientes com idade > 70 anos, de ambos os sexos, com estenose aórtica grave, no período de julho de 2010 a outubro de 2016.

Resultados: Foram incluídos 20 pacientes com idade média de 82,7 ( $\pm 4,98)$, com predominância do sexo feminino (60\%). Houve prevalência de implante da válvula Corevalve ${ }^{\circledR}(84 \%)$ e Edwards SAPIEN ${ }^{\circledR}$ (16\%); o planejamento, organização e preparo da sala pela equipe de enfermagem variou de 60 minutos nos primeiros procedimentos a 30 minutos atualmente; a equipe é composta por um enfermeiro e dois técnicos de enfermagem (1 na instrumentação); o tempo do procedimento variou de 180 a 50 minutos; a implementação do check-list foi aplicada em 100\% dos casos. Até outubro de 2016, já foram realizados 9 procedimentos, correspondendo a $45 \%$ de todos os procedimentos já feitos.

Conclusão: Esses resultados permitem compartilhar com a comunidade acadêmica a evolução desse procedimento em uma instituição pública. O planejamento, a organização e o check-list aplicado em 100\% da casuística permitem alcançar resultados plenos com mais segurança. 


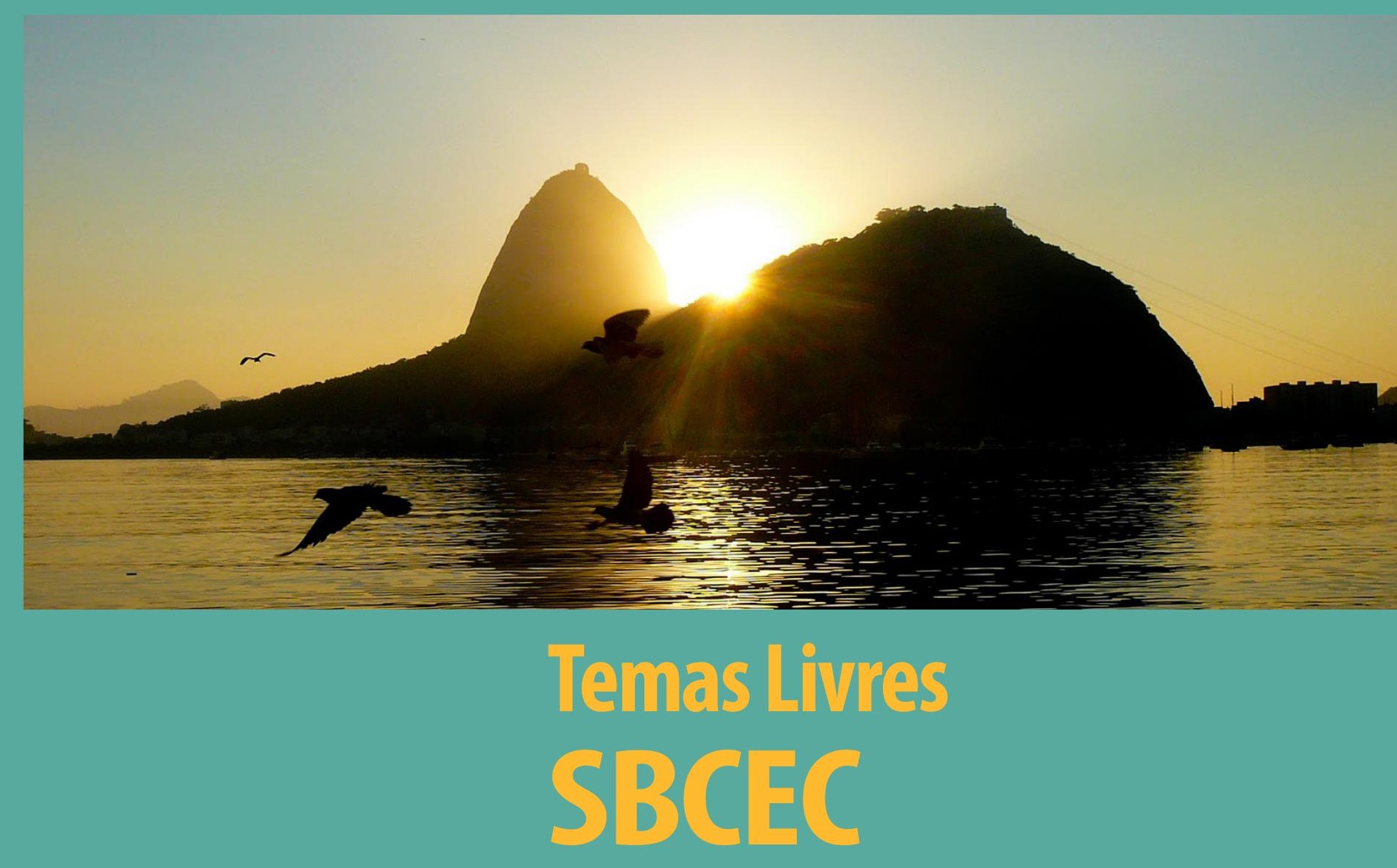




\section{TT $01 \bullet 10 \mathrm{~h} 30-12 \mathrm{~h} 15$}

\section{Padronização de serviço de circulação extracorpórea em cirurgia cardíaca adulto e pediátrica}

Alexsandro Rodrigues de Oliveira

O Complexo Hospitalar Irmandade Santa Casa de Misericórdia de Porto Alegre possui 7 hospitais de diferentes especialidades médicas, proporcionando várias áreas de atuação para o perfusionista clínico. Mas é na área cardíaca que temos a maior demanda para este profissional. Entre adulto e pediatria, temos cerca de 750 cirurgias/ano com extracorpórea; o que se faz necessário iniciar a implementação de uma padronização do serviço por esta especialidade.

O Objetivo deste trabalho é Implementar uma padronização do Serviço de Circulação Extracorpórea no Complexo Hospitalar Santa Casa de Porto Alegre nas áreas de cirurgia cardíaca adulto e pediátrica.

Usamos como ferramenta de gestão da qualidade do serviço de circulação extracorpórea do Complexo da Santa Casa, identificando prioridades, envolvendo todas as pessoas relacionadas com a rotina de cirurgia cardíaca e circulação extracorpórea. Procuramos elaborar de forma coletiva, com atualização periódica e disponibilização no site da instituição de todo o material. A padronização, além de ser uma destas exigências, é uma forma de qualificar o serviço de extracorpórea, auxiliar na formação do perfusionista e atualizar o profissional já atuante

\section{TT $02 \bullet 10 \mathrm{~h} 30-12 \mathrm{~h} 15$}

\section{Novas práticas na circulação extracorpórea: o uso da del Nido Cardioplegia}

Damaris Vieira Braga Carvalho

A cardioplegia é um método de proteção miocárdica utilizada durante a cirurgia cardíaca. No início dos anos de 1990, um grupo liderado por Pedro del Nido, Hung Cao-Danh, Eric Sommers e Akihiko Ohkado criou uma formulação que ficou conhecida como del Nido Cardioplegia para ser utilizada em cirurgias de crianlas no Boston Children's Hospital, devido a imaturidade do coração delas. Originalmente criada para cirurgia de crianças, passou a ser utilizada nas cirurgias de adultos devido a sua praticidade e baixo custo e necessidade apenas de uma única infusão. Foi realizada uma revisão integrativa de artigos publicados nos últimos 10 anos para descrever a história, uso e resultados desta cardioplegia. Foi visto que esta solução tem ganhado espaço dentro das equipes cirúrgicas podendo ser utilizada nos diversos serviços de saúde, tanto público quanto privado, devido ao baixo custo de produção, praticidade na manipulação e bons resultados pós-cirúrgicos.

\section{TT $03 \bullet 10 \mathrm{~h} 30-12 \mathrm{~h} 15$}

\section{Relato de caso: utilização de circuito híbrido de ECMO como ponte para o tratamento}

Daniela Pavão, Stevan Krieger Martins, Nadia Maria Rocha Mendes, Kadige Jamil el Kadri

Alguns pacientes quando submetidos à cirurgia cardiovascular podem sofrer alterações hemodinâmicas ou respiratórias graves e nestes casos, o suporte cardiopulmonar com uso de bomba centrífuga acoplada a um oxigenador, pode ser indicado para o tratamento, possibilitando a recuperação de órgãos alvo ou em determinados casos ponte para transplante.

Este suporte cardiopulmonar é denominado ECMO (Extracorporeal Membrane Oxygenation) e ele não irá curar o paciente, porém irá oferecer um tempo, um período de repouso para que os órgãos se recuperem.

Muitas vezes o circuito convencional de ECMO não está disponível na instituição onde esta sendo realizando o procedimento, portanto a montagem de um circuito híbrido pode ser a única alternativa encontrada para salvar a vida do paciente, enquanto não se adquire o circuito ideal 


\title{
APRESENTAÇÃO DE TEMAS LIVRES - SBCEC
}

\section{TT $04 \cdot 10 \mathrm{~h} 30-12 \mathrm{~h} 15$}

\section{Complicações que podem ocorrer no pós-operatório de cirurgia cardiovascular com circulação extracorpórea}

\author{
Juliana Martins Provenciato, Andréia Cristina Passaroni
}

A cirurgia cardíaca com circulação extracorpórea (CEC) representou uma das grandes conquistas médicas e da área biológica no século XX, criando novas possibilidades para a cura de doenças cardíacas, jamais imaginadas na primeira metade do século passado, entretanto a CEC está associada a inúmeras complicações clínicas em pós-operatório de cirurgia cardiovascular. A cirurgia cardiovascular com CEC oferece vantagens, porém com importantes repercussões, alterando mecanismos fisiológicos dos pacientes levando-os a um estado crítico pós-operatório que implica a necessidade de cuidados intensivos especializados. Este estudo, por meio de análise retrospectiva de artigos publicados na Medline via Pubmed, teve como objetivo realizar uma revisão da literatura sobre complicações que podem ocorrer no pós-operatório de cirurgia cardiovascular com circulação extracorpórea.

\section{TT $05 \bullet 10 \mathrm{~h} 30-12 \mathrm{~h} 15$}

\section{Checklist na assistência circulatória mecânica: uma ferramenta para cirurgia segura Rafaela Mourão Redon Fernandes Casa Nova}

Introdução: A circulação extracorpórea é um procedimento de alta complexidade, necessitando atenção e rotina do perfusionista para ser feita com êxito e livre de acidentes. A elaboração de um checklist para a checagem dos dados necessários à assistência circulatória mecânica foi o objeto investigado.

Objetivo: propor um checklist para auxiliar o perfusionista na sistematização de suas ações e garantir a segurança do paciente no âmbito da assistência circulatória mecânica.

Métodos: foi feita uma busca em livros e artigos específicos de Circulação Extracorpórea contendo a citação de todos os equipamentos e dados necessários para a perfusão cardíaca; a segunda etapa foi o estudo teórico, com base em revisão de literatura e a terceira etapa foi a elaboração do Checklist.

Resultados: foram selecionados os tópicos referentes a checagem de itens necessários a uma perfusão segura. Assim foram definidos os seguintes temas a serem contemplados no checklist: dados do paciente, história patológica e cirurgia proposta, equipamentos necessários para a circulação extracorpórea, medicações utilizadas no procedimento e ações a serem tomadas após o término do procedimento.

Conclusão: o checklist é importante para a garantia da segurança do paciente e instituição da rotina do perfusionista, levando à qualidade do atendimento.

\section{TT $06 \bullet 10 \mathrm{~h} 30-12 \mathrm{~h} 15$}

\section{Cardiopulmonary bypass (CPB) care of patients with Sickle Cell Disease submited to cardiac surgery}

Raquel Christine Kruger Miranda, Ronaldo David da Costa, Orlando Carlos Belmonte Wender

Patients with sickle cell disease (SCD) undergoing cardiac surgery with cardiopulmonary bypass (CPB) are at greater risk of perioperative complications. Modifications of routine and application of advanced techniques of surgery, monitoring, anesthesia and specially perfusion are required for successful results. The aim of this present study is to report a patient case and present a brief bibliographical review of management strategies that can be used by perfusionists in patients undergoing surgeries with CPB while dealing with SCD. The patient, a 30-year-old man of African origin with homozygous sickle cell anemia, was submitted to an aortic valve replacement plus an ascendant aorta aneurism repair surgery with CPB. Careful monitoring of parameters of hematology, hemodynamic, oxygenation, acid-basis and anesthesia parameters was mandatory. Also important in this condition is attention to the need of exchange transfusion, the choice of valve used, the $u$ se of hemofiltration and Cell-Saver, as well as avoiding the patient's stress, acidosis, hypoxia, low blood flow and hypothermia. With advances in diagnostic, anesthetic and surgical techniques, patients with sickle cell disease should be safely submitted to cardiac surgery with CPB 


\section{TT $07 \bullet 10 \mathrm{~h} 30$ - $12 \mathrm{~h} 15$}

Uso de membrana de oxigenação extracorpórea em caso de pós transplante como ponte para recuperação

\section{Alana Michele da Rocha Melo}

A indicação do uso de um suporte de membrana de oxigenação extracorpórea deve ser critério de escolha para pacientes com falência na saída de circulação extracorpórea quando ocorre a falha do enxerto em pós transplante cardíaco. O trabalho trata-se de um estudo de caso com paciente pós transplante cardíaco no qual se obteve total recuperação da paciente. 


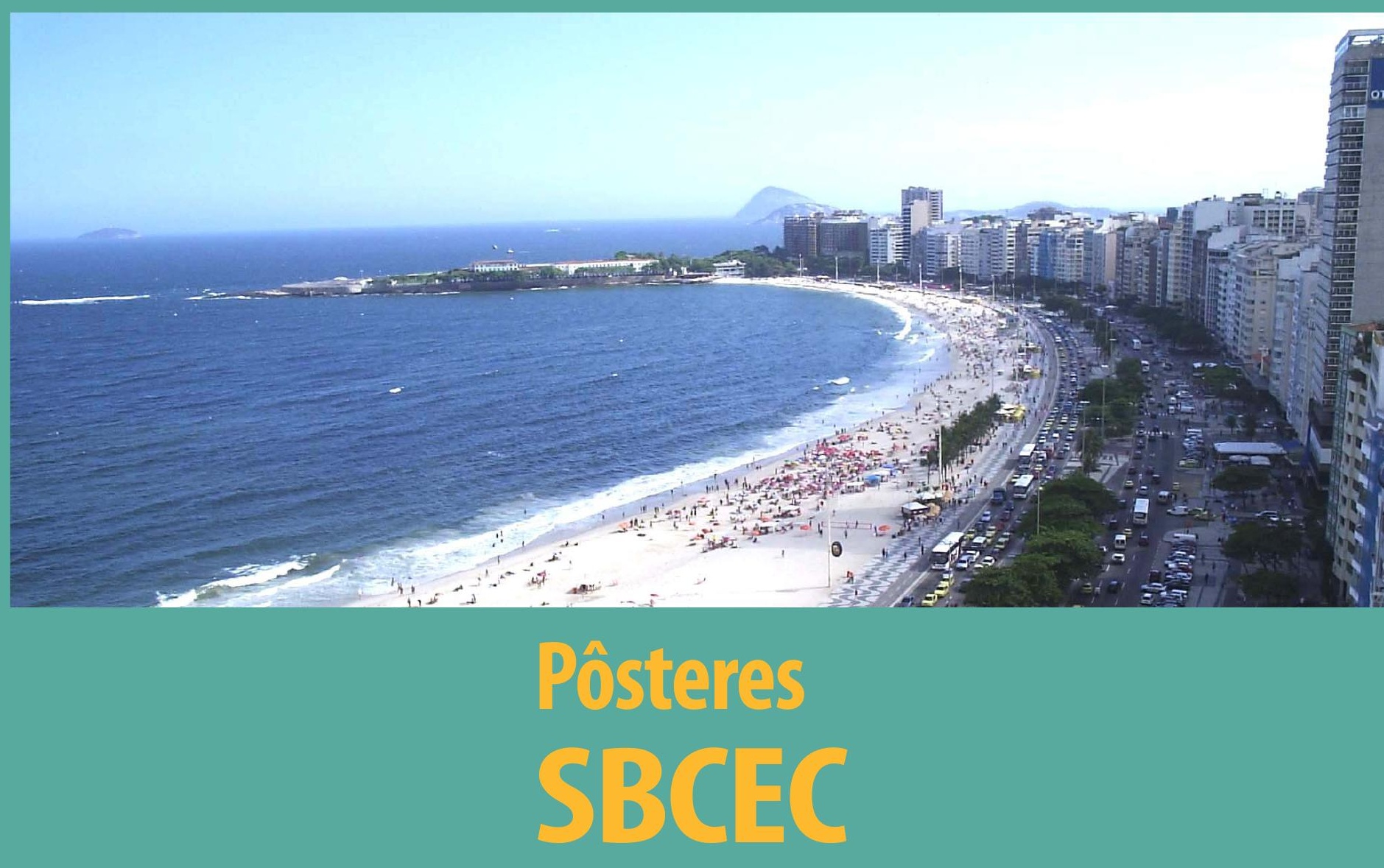




\title{
PO 01
}

\section{Bases para elaboração de checklist em circulação extracorpórea}

\author{
Andrelise Maria Nicoletti, Cláudia Zamberlan
}

\begin{abstract}
O presente estudo visa identificar os principais eventos adversos em circulação extracorpórea durante as cirurgias cardiovasculares, bem como a elaboração de um checklist no processo de implantação de cirurgia segura nesta especialidade. Configura-se como uma revisão narrativa de literatura constituída de referências científicas nacionais e internacionais. Foram selecionadas doze referências entre 2007 a 2015. Evidenciou-se que o desenvolvimento da cirurgia cardiovascular é baseado no progresso da circulação extracorpórea. Os eventos adversos na circulação extracorpórea durante o procedimento cirúrgico podem ocasionar complicação grave e/ou irreversível ao paciente. Nesse sentido, foi elaborado um checklist em prol da cultura de segurança em ambiente cirúrgico. Conclui-se que os estudos nessa área ainda são limitados, todavia, tornou-se perceptível que a implantação de checklist direcionado pode contribuir para a consolidação da segurança do paciente durante as cirurgias cardiovasculares com uso de circulação extracorpórea.
\end{abstract}

\section{PO 02}

\section{Revisão da literatura: isquemia-reperfusão do miocárdio e proteção miocárdica}

Caroline Nonis Zucoloto; Sandra Regina Ormenesi; Natalia Cristina Andreata; Flávia Cristina Gomes Alves; Alfredo Inácio Fiorelli, Fabio Biscegli Jatene

Introdução: A isquemia induz no miocárdio profundas alterações podendo levar à desorganização na sua estrutura morfofuncional do cardiomiócito, podendo ocasionar até a morte celular. A reperfusão tem por objetivo a reversão dessas alterações e evitar a lesão irreversível, todavia, tem-se observado que muitas vezes os danos são maiores do que a própria isquemia. Diferentes técnicas de proteção miocárdica têm sido desenvolvidas com o intuito de minimizar a falência miocárdica pós-cardiotomia.

Objetivo: Realizar uma revisão bibliográfica à respeito da isquemia e reperfusão miocárdica.

Método: Vinte e cinco artigos foram selecionados no período de Março a Dezembro de 2016, com base nos descritores: Lesão de Reperfusão; Isquemia Miocárdica; Pré-condicionamento Isquêmico; Pós condicionamento Isquêmico, tendo como base de dados a Scientific Library Online (SCIELO) e livros publicados sobre o tema.

Resultados: Os efeitos indesejáveis do fenômeno de isquemia e reperfusão podem ser minimizados com a adoção de diferentes métodos de proteção miocárdica, por meio de quatro processos: (1) a utilização da hipotermia, que resulta em redução da atividade metabólica celular; (2) a infusão de soluções cardioplégicas, que induzem alta concentração de potássio extracelular, causando a despolarização das membranas celulares e como consequência, diminuindo a capacidade de contração do miócito e seu consumo de oxigênio; (3) o pré-condicionamento isquêmico, que consiste em parada temporária do fluxo sanguíneo, prévios à prolongada oclusão arterial; e (4) o pós-condicionamento isquêmico, que consiste em curtos períodos de isquemia durante os minutos iniciais da reperfusão, após oclusão arterial.

Conclusão: Os danos miocárdicos causados pela isquemia são severos. Os efeitos deletérios produzidos no reestabelecimento do fluxo sanguíneo, todavia, são maiores. Estudos apontam hipotermia, cardioplegia e pré e pós condicionamento como importantes métodos de proteção miocárdica. São necessários, porém, mais estudos acerca do tema, a fim de elucidar todos os seus benefícios.

\section{PO 03}

\section{Atuação da enfermagem na cirurgia pediátrica de cardiopatia congênita}

Manoel de Sousa Martins, Claudia Maria Messias

Introdução: As cardiopatias congênitas são definidas como anormalidades, tanto na estrutura como na função cardiocirculatória, logo após o nascimento. Foram desenvolvidas inúmeras técnicas cirúrgicas para manter essas crianças vivas, podendo chegar até mesmo ao transplante cardíaco. Através dessa evolução cientifica é necessária qualificação da enfermagem tendo em vista que acompanhamento das fases pré, trans e pós operatória estão relacionados à assistência de enfermagem. 


\title{
EXPOSIÇÃO DE PÔSTERES - SBCEC
}

Objetivo: Descrever a atuação da enfermagem no pré, trans e pós-operatório através do processo de sistematização da assistência na cardiopediatria.

Métodos: Revisão bibliográfica buscando determinar as responsabilidades da enfermagem em todo o processo da cirurgia pediátrica de cardiopatia congênita.

Resultados: As intervenções de enfermagem no pré, trans e pós-operatório auxilia na prevenção e diagnóstico precoce das complicações, favo recendo a recuperação da criança e diminuindo o tempo de permanência no hospital.

Discussão: Antes mesmo da indicação da cirurgia, o enfermeiro pode realizar, no ambulatório, a consulta e avaliação dessa criança. Sendo o tratamento da cardiopatia feito por cirurgia o profissional agendará a reunião multiprofissional pré-operatória onde tirará as dúvidas. A criança será internada no dia anterior a cirurgia, onde o enfermeiro realizará avaliação. Durante a cirurgia a enfermagem atua no planejamento e aquisição de equipamentos e materiais e na prevenção e controle da infecção cirúrgica. Já no pós-operatório a assistência é voltada para a recuperação. Estímulo à aceitação de dieta, avaliação da cicatrização da incisão cirúrgica e avaliação do estado geral, como objetivo de incorporar a criança novamente ao convívio familiar e social.

Conclusão: A enfermagem tem um papel significativo na melhora da criança que realizou a cirurgia de cardiopatia congênita. Pois, esse profissional acompanhará o paciente em todas as etapas do tratamento. Sendo assim, possuindo competências que auxiliará na reabilitação dessa criança.

\section{PO 04}

\section{Cuidados de enfermagem ao paciente com cardiopatia: um relato de caso}

Márcia Gomes de França, Letícia Machado de Sousa, Patrícia Tavares de Morais, Bianca de Melo França, Silvan Alcantara da Silva

Cardiopatias são quaisquer doenças que atingem o coração e o sistema sanguíneo. O presente trabalho tem como objetivo relatar a assistência de enfermagem ao paciente com cardiopatia. Paciente, 30 anos, masculino. Deu entrada na unidade hospitalar queixando-se de dispneia e algia precordial, sendo diagnosticado com cardiopatia. Através do levantamento dos problemas do paciente foram identificados os seguintes diagnósticos de enfermagem: risco de função cardiovascular prejudicada, ventilação espontânea prejudicada, estilo de vida sedentário e insônia. É de fundamental importância que a enfermagem se aproprie da sistematização da assistência de enfermagem para prestar um cuidado qualificado e humanizado.

\section{PO 05}

\section{Tratamento farmacológico nas valvulopatias: um relato de caso}

Silvan Alcantara da Silva, Márcia Gomes de França, Letícia Machado de Sousa, Patricia Tavares de Morais, Francisca Hermilene Pedro Rodrigues

As valvulopatias são doenças que acometem as válvulas responsáveis pelo bombeamento de sangue entre as câmeras cardíacas. 0 estudo tem como objetivo avaliar interação medicamentosa de uma prescrição de um paciente com valvulopatia. Realizou-se um levantamento das prescrições médicas no prontuário do paciente P.H.G, 31 anos, residente em Fortaleza/CE e diagnosticado com Valvulopatia, analisou-se os aprazamentos e medicações feitas pela enfermagem e possíveis interações medicamentosas.

Foi identificada interação farmacodinâmica por mecanismo de sinergismo entre a losartana e a furosemida, ambas drogas hipotensoras.

É necessário que a enfermagem tenha conhecimento farmacológico para identificar possíveis interações medicamentosas, permitindo modificações no aprazamento das medicações e refletindo na melhoria da assistência prestada ao paciente.

\section{PO 06}

\section{O implante da valva aórtica com o transcatéter}

\author{
Vanessa Cristina Freitas Moreno, Ana Flavia Barbosa da Silva
}

Introdução: A calcificação degenerativa da valva aórtica é uma das causas mais comuns de estenose aórtica nos países desenvolvidos e com mais frequência em indicação da substituição da valva aórtica. O tratamento padrão consiste numa prótese 
biológica ou mecânico com mortalidade operatória em aproximadamente 4\%. Apesar deste resultado, alguns pacientes possuem morbimortalidade elevada, com fatores de riscos como: idade avançada, operações prévias, fragilidade biológica entre outros. Nos últimos tempos, o implante de uma bioprótese aórtica tem proposto o mesmo objetivo, porém com resultados mais consistentes e mais otimistas, o aprimoramento destes dispositivos como melhorias desde a redução do calibre dos introdutores tem fornecido um resultado significativo.

Com isso, a troca valvar aórtica com o transcatéter é um procedimento rotineiro e minimamente invasivo na qual parece ser alternativa diminuindo a morbimortalidade.

Objetivo: Avaliar a segurança e eficácia do procedimento.

Métodos: Revisão literária através de pesquisas de artigos de diversas fontes incluindo PubMed e Scielo. Com isso, nos forneceu um conjunto de referências para esta análise, onde foi possível comparar resultados e opiniões de profissionais da área referente a prótese, o procedimento e a qualidade de vida dos pacientes envolvidos.

Resultados: De um modo geral, através de nossa pesquisa foi constatado que o transcatéter é um procedimento possível e satisfatório com resultados muito atrativos aos pacientes de doenças valvares, capaz de fornecer melhora estrutural e funcional cardíaca. Ainda são necessários muitos estudos para se determinar a qualidade de vida e sobrevida a longo prazo.

\section{PO 07}

\section{Uso de ECMO na cardiopediatria}

Wagner Santos Knoblauch, Mellyssa Matos de Castro Lima, Tathiane Aquaroni Davoglio, Ana Luiza Paulista Guerra, Lily Emilia Montalvan Rabanal, Omar Pozo Ibañes, Paulo Chaccur, Glaucio Furlanetto

Introdução: O uso de oxigenação por membrana extracorpórea (ECMO) para suporte circulatório cardiopulmonar é cada vez mais frequente e crucial nos centros de terapia intensiva de cardiopediatria, especialmente no manejo pós-operatório de cardiopatias congênitas complexas.

Objetivo: Descrever os avanços no uso de ECMO em uma instituição de referência para crianças cardiopatas.

Métodos: Análise retrospectiva de diagnóstico, indicação, duração, desmame, complicações e sobrevida dos pacientes pediátricos submetidos à ECMO.

Resultados: Ao todo, 29 pacientes foram submetidos à ECMO no período de janeiro de 2013 a dezembro de 2016 , com idade entre 18 dias de vida e 14 anos e peso entre 2,8 Kg a 56 Kg. A falência ventricular esquerda e/ou direita pós-cardiotomia foi a indicação mais frequente de ECMO (55,2\%), seguida de falência ventricular a partir do $1^{\circ}$ dia de pós-operatório (20,7\%), falência pulmonar (13,8\%) e ponte para transplante cardíaco (10,3\%). A canulação do tipo venoarterial foi utilizada em todos os pacientes, com duração entre 1 hora a 34 dias. As complicações mais observadas foram politransfusão (100\%), plaquetopenia (100\%), injúria renal aguda dialítica (82,7\%), sangramento com revisão da hemostasia (65,5\%) e coma irreversível (41,3\%). Em relação ao desfecho, o desmame da ECMO foi possível em 16 pacientes (55,1\%), com 7 altas hospitalares (24,1\%). Cerca de metade dos óbitos, durante ou após a ECMO, ocorreu em decorrência de choque cardiogênico por falência ventricular não recuperada.

Discussão: $\mathrm{O}$ uso de ECMO na cardiopediatria configura-se em um incremento substancial no arsenal terapêutico, com impacto direto no manejo e sobrevida dos pacientes com defeitos cardíacos, notadamente naqueles com diagnósticos e intervenções de maior complexidade.

Conclusão: O aperfeiçoamento permanente dos profissionais e equipamentos da ECMO é fundamental para a obtenção e manutenção de resultados favoráveis.

\section{P0 08}

\section{Sistematização da assistência de enfermagem a gestante cardiopata: um relato de caso}

Francisca Hermilene Pedro Rodrigues, Márcia Gomes de França, Letícia Machado de Sousa, Silvan Alcântara da Silva, Patrícia Tavares de Moraes

As síndromes coronarianas representam uma das principais causas de morbimortalidade em todo o mundo, sendo a valvulopatia mitral uma das mais comuns. Este trabalho tem como objetivo relatar a assistência de enfermagem a gestante cardiopata. Paciente 


\title{
EXPOSIÇÃO DE PÔSTERES \\ - SBCEC
}

M.F.M, 43 anos, feminino, G6P4A1. Há 3 anos possui prótese biológica em valva mitral, e portadora de hipertensão pulmonar. Através da investigação do quadro clinico da paciente foram identificados os seguintes diagnósticos de enfermagem: Falta de adesão ao acompanhamento de pré-natal, comportamento de saúde propenso a risco e risco de função cardiovascular prejudicada. É fundamental que os profissionais de enfermagem se apropriem do processo de enfermagem (PE), que são utilizados como meio de prestar uma assistência qualificada e humanizada.

\section{PO 09}

\section{Biomarcadores de lesão renal aguda pós cirurgia cardíaca com circulação extracorpórea}

\author{
Diego Faria Marques Ferreira; Flávia Gomes Alves; Alfredo Inácio Fiorelli
}

\begin{abstract}
Introdrução: O termo "doença renal aguda" engloba várias condições de redução da função renal, com altas taxas de morbidade e mortalidade (HAASE; HAASE-FIELITZ, 2011). Níveis de creatinina sérica são dependentes de vários outros fatores não renais e aumentam 24 a 48 horas após o evento prejudicial para o rim. Existe hoje em dia uma variedade de novos biomarcadores de lesão renal, que precede clinicamente a lesão por vários dias ou semanas (HAASE; HAASE-FIELITZ, 2011). Os biomarcadores mais promissores, como a lipocalina associada com gelatinase de neutrófilos (NGAL), molécula de lesão renal 1 (KIM-1) e interleucina-18 (IL-18), têm sido investigadas a tal ponto que as revisões sistemáticas e meta-análises surgiram aprovando seu reconhecimento (SCHUH et al., 2016). ANGAL é um biomarcador sensível do rim e foi investigada através de uma gama de cenários clínicos de IRA, incluindo a cirurgia cardíaca (MISHRA et al., 2005) (HAASE-FIELITZ et al., 2009) (FAN et al., 2015) e sepse (VIVES et al., 2012). Resultados confirmam que NGAL no plasma e na urina são excelentes preditores para insuficiência renal aguda após CEC em pacientes neonatais e pediátricos. Além disso, foi associada à gravidade da insuficiência renal aguda e ao prognóstico clínico. (KRAWCZESKI et al., 2011).
\end{abstract}

Objetivo: Firmar a importância de novos biomarcadores renais que são preditores da lesão propriamente instalada, que aumentam seus níveis séricos logo após o término da cirurgia cardíaca com CEC.

Discussão: Um único biomarcador para IRA pode não fornecer informações completas. Estudos recentes têm descoberto outros biomarcadores para lesão renal aguda (KRAWCZESKI et al., 2011).

Conclusão: A cirurgia cardíaca com CEC leva à liberação de diversos biomarcadores renais para a circulação, inclusive os que são liberados antes lesão renal instalada.

\section{PO 10}

\section{Fluxo pulsátil induzido pelo balão intra-aórtico durante circulação extracorpórea. Revisão de literatura}

\author{
Gabriela Cozin Aragão
}

Diferentes técnicas e dispositivos para o emprego de fluxo pulsátil durante a circulação extracorpórea (CEC) foram tentados para mimetizar o padrão de fluxo fisiológico, contudo, todos sem sucesso. Os principais fatores impeditivos referem-se à complexidade dos sistemas e o traumatismo aos elementos sanguíneos impostos por eles. Todavia, os benefícios do emprego da contra pulsação têm sido amplamente ressaltados em diferentes investigações clínicas. Desta forma, tem-se ampliado o número de pacientes que são encaminhados para a cirurgia cardíaca com a instalação prévia do balão intra-aórtico (BIA). Poucos centros têm por hábito manter o BIA ligado com o objetivo de gerar fluxo pulsátil. Assim a presente investigação teve por objetivo realizar uma análise crítica sobre a manutenção do BIA ligado durante a CEC. Para isso, foi realizada uma de revisão da literatura no PubMed, a partir das palavras chave: Balão intra-aórtico; fluxo pulsátil e Circulação extracorpórea, que resultou na seleção de 10 artigos randomizados, publicados entre 1975 e 2013, os quais avaliaram os efeitos clínicos do uso do fluxo pulsátil induzido pelo BIA durante a CEC. A partir dos resultados apresentados pelos trabalhos selecionados, a utilização do fluxo pulsátil induzido pelo BIA exerce benefícios sobre a perfusão tecidual em relação ao fluxo continuo e nenhum efeito deletério foi associado a utilização do BIA ligado durante a CEC, entretanto a maior parte estudos apresentados não mostram diferenças significativas na morbidade e na mortalidade associada a utilização desta abordagem. Desta forma conclui-se que apesar de haver controvérsias quanto às vantagens do emprego do fluxo pulsátil produzido pelo BIA durante a CEC, esta prática trata-se de um terreno fértil para investigações futuras, tendo em vista a possibilidade de se obter um padrão de fluxo semelhante ao fisiológico durante a CEC. 


\section{PO 11}

\section{A importância da assistência de enfermagem no pós-operatório imediato da cirurgia de transplante cardíaco}

Manoel de Sousa Martins, Sandra Cristina de Sousa Borges Silva, Raquel Andrade Miranda Rangel

Introdução: O transplante cardíaco é indicado para o paciente com insuficiência cardíaca congestiva grave e refratária ao tratamento clínico. Exigindo do profissional de enfermagem uma assistência especifica, com qualidade e realizando reavaliações periódicas. No período pós-operatório imediato, o enfermeiro deve traçar inúmeras intervenções, preocupando-se, principalmente, com as infecções. Devido à terapia imunossupressora que tem o objetivo de prevenir a rejeição do enxerto.

Objetivo: Descrever a relevância da assistência de enfermagem nos primeiros dias pós-cirúrgico do transplante cardíaco.

Métodos: Revisão bibliográfica estimando os cuidados de enfermagem no período pós-operatório imediato aos pacientes de transplante cardíaco destacando a sua valia. Resultado: A assistência exercida no pós operatório imediato do transplante cardíaco tem grande significância na evolução do paciente. A partir dessa assistência é possível traçar intervenções que venham estabilizá-lo após cirurgia, além de evitar complicações.

Discussão: A equipe de enfermagem nos primeiros dias após a realização do transplante cardíaco tem como responsabilidade prestar cuidados ao paciente para que seu organismo reestabeleça seu funcionamento a partir do órgão transplantando, são eles: manutenção adequada da função respiratória, estabilização hemodinâmica e monitorização dos sinais vitais. Além disso, é necessário verificar as possíveis complicações. Podemos destacar as infecções. Intercorrência muito comum devido a realizar da terapia imunossupressora, além de aparelhos invasivos como os drenos torácicos, tubo endotraqueal, cateteres venosos e sonda vesical. Sendo assim, o enfermeiro deve traçar intervenções tanto preventivas quanto de controle para tal paciente.

Conclusão: O transplante cardíaco tem como objetivo trazer uma sobrevida de qualidade ao paciente transplanta do, porém para isso é necessário que a enfermagem exerça os cuidados necessários responsáveis pela reabilitação do indivíduo, desde o período do pós operatório imediato até o tardio, através de consultas de acompanhamento.

\section{PO 12}

\section{Importância de profissionais qualificados na realização de circulação extracorpórea}

Kelly Cristiane Gabriel de Almeida

A perfusão ou circulação extracorpórea (CEC) é o procedimento no qual se substituem temporariamente as funções dos pulmões e do coração, deixando-os excluídos da circulação, através de máquinas, aparelhos, circuitos e técnicas. Ela consiste em desviar o sangue do coração direto para um sistema mecânico que o filtra, promove a sua oxigenação e regula a sua temperatura, substituindo as funções dos pulmões. Quando transformado em sangue arterial, o sangue regressa à circulação sistêmica com o auxílio de uma bomba, que substitui a função do coração. Dessa forma, a CEC não apenas substitui as funções cardiopulmonares, mas, ao mesmo tempo, preserva a integridade celular, a estrutura, a função e o metabolismo dos órgãos e dos sistemas do indivíduo durante operações complexas e demoradas.

Por se tratar de uma técnica extremamente complexa e de vital importância para o paciente, precisa ser realizada por um profissional altamente qualificado, o perfusionista, cuja função é extremamente ativa e precisa. Ele trabalha junto com a equipe que atua no bloco cirúrgico e é constituída por cirurgiões, anestesistas e enfermeiros 


\section{EXPOSIÇÃO DE PÔSTERES - SBCEC}

\section{PO 13}

\section{Gestante submetida à correção de disfunção de bioprótese mitral com circulação extracorpórea em caráter de urgência. Relato de caso}

Rebeca Sampaio Goes Cavalcante, Élida Carvalho Rezende, Flavia Cristina Gomes Alves, Carlos Manuel de Almeida Brandão, Ellington Veronese, Pablo Maria Alberto Pomerantzeff, Alfredo Inácio Fiorelli, Fabio Biscegli Jatene

Introdução: As doenças cardiovasculares são complicações presentes em 0,2 a 4\% das gestações e em aumento, conforme Diretriz da Sociedade de Cardiologia Europeia de 2011. Quando o tratamento clínico não é suficiente, a operação cardiovascular é recomendada. A utilização da circulação extracorpórea (CEC) aumentou a incidência dos riscos obstétricos e fetais, sendo que o conhecimento aprofundado da equipe cirúrgica sobre as repercussões da mesma, permite o aprimoramento do processo cirúrgico para reduzir estes riscos.

Objetivo: Descrever o manejo da CEC em uma paciente primigesta de 30 semanas submetida à troca de bioprótese mitral por disfunção grave.

Relato do caso: Mulher de 17 anos, primigesta na 30a semana que foi submetida à correção cirúrgica devido à presença de disfunção de bioprótese mitral com calcificação grave. Inicialmente, houve a programação de acelerar a maturação fetal, contudo por causa do agravamento clínico foi submetida à operação de urgência com CEC, na qual tomaram medidas para redução dos riscos maternos e fetais.

Conclusão: Gestante submetida à operação cardiovascular de urgência com CEC pode ser cumprida com uma preparação estruturada e com a participação de equipe multidisciplinar.

\section{PO 14}

\section{Protocolo de circulação extracorpórea utilizado na instalação e manutenção de um dispositivo de assistência biventricular - Estudo de caso}

Wiliane Resende sousa

Atualmente, o tratamento clínico da insuficiência cardíaca grave atingiu níveis elevados de excelência. Entretanto, mesmo com o progresso da ciência, os estudos revelam que ainda temos um prognóstico ruim (falência Cardíaca), com elevada morbimortalidade. Isso posto, o tratamento da falência cardíaca têm sido focado e aprimorado com o advento da bioengenharia, desenvolvendo novas tecnologias como é o caso dos dispositivos de assistência circulatória mecânica, que proporcionam melhora da qualidade e expectativa de vida nesse grupo. O presente trabalho é um estudo de caso que descreve o protocolo utilizado em um serviço de circulação extracorpórea para a instalação e manutenção de dispositivo de assistência biventricular em uma criança de 07 anos, transplantada do coração há 03 anos e 07 meses, com diagnóstico de rejeição aguda do órgão transplantado. O caso relatado aconteceu em um hospital de referência em cardiologia e cirurgia cardiovascular pediátrica vinculado ao Sistema Único de Saúde (SUS), em Fortaleza - Ceará, no mês de fevereiro de 2016. Buscando-se destacar a experiência do protocolo de instalação e manutenção do dispositivo de assistência biventricular.

\section{PO 15}

\section{Mini circuito de circulação extracorpórea em fetos de ovinos}

Ana Paula Noronha, Renato S. Assad, Petronio G. Thomaz, Marcelo Guedes, Fernando Zanoni, Ana Cristina Aliman, Marcelo Vaidotas, Giuliano P. da Silva, Marcelo B. Jatene, Alfredo I. Fiorelli, Fabio B. Jatene, Luiz Felipe P. Moreira

Objetivo: A cirurgia cardíaca fetal pode representar a alternativa terapeutica mais lógica para certas cardiopatias congênitas complexas que apresentam elevada morbi-mortalidade intra-uterina ou após o nascimento. A disfunção placentária representa a principal limitação da circulação extracorpórea (CEC) fetal. Descrevemos um mini circuito inovador de CEC fetal para aplicação na cirurgia cardíaca intrauterina. 
Métodos: Foram utilizadas doze fetos de ovelhas (raça mista), com cerca de $90 \%$ da idade gestacional, divididos em dois grupos (Pesos: Controle: $2.683 \mathrm{~g} \pm$ 189,0 g; Mini CEC: $2.979 \mathrm{~g} \pm$ 415,8 g). O grupo Mini CEC foi colocado em CEC durante 30 minutos, utilizando o Rotaflow como bomba e a placenta como oxigenador in vivo do circuito (não foi utilizado oxigenador). $O$ perfusato foi composto de ringer simples $(61,33 \mathrm{ml} \pm 6,57 \mathrm{ml})$. Os fetos foram monitorados durante duas horas após a CEC.

Resultados: O fluxo máximo atingido durante a CEC fetal foi de 137,0 $\pm 16,3 \mathrm{ml} / \mathrm{min} / \mathrm{kg}$ de peso fetal. A tabela 1 mostra os resultados da pressão arterial média sistêmica $(\mathrm{mmHg})$, gasometria arterial, bicarbonato e lactato dos fetos nos dois grupos

\title{
PO 16
}

\section{Extracorporeal Membrane Oxigenation (ECMO): uma revisão sistemática de literatura sobre suas principais complicações}

\author{
Andrelise Maria Nicoletti, Cláudia Zamberlan
}

\begin{abstract}
A Extracorporeal Membrane Oxigenation (ECMO) é considerada uma das mais complexas terapias de assistência circulatória, sendo indispensável um trabalho multidisciplinar durante o seu emprego. Apesar de ocorrer um aumento significativo nos últimos anos nas taxas de utilização desse suporte hemodinâmico com mais segurança, ainda podem ser observadas complicações. Nesse contexto, o presente estudo tem como objetivo identificar as principais complicações durante a ECMO. Configura-se em uma revisão sistemática de literatura constituída de artigos científicos nacionais e internacionais sobre a temática indexados no período de 2010 a 2016. Foram selecionados onze artigos científicos totalizando 7.218 pacientes, dos quais 40,31\% apresentaram complicações renais; 36,89\% complicações hematológicas; 16,2\% complicações vasculares; 13,98\% infecção; 8,85\% disfunção neurológica e 20,16\% complicações mecânicas com o circuito de ECMO. O desenvolvimento de condutas que estimulem a cultura de segurança é uma exigência em instituições hospitalares. Nesta perspectiva, a utilização de medidas preventivas de caráter multidisciplinar pode contribuir para a consolidação da segurança do paciente durante o suporte circulatório mecânico com ECMO.
\end{abstract}

\section{PO 17}

\section{A importância do sulfato de magnésio (MgSO4) na cirurgia cardíaca com circulação extracorpórea}

\section{Diego Faria Marques Ferreira, Flávia Cristina Gomes Alves, Alfredo Inácio Fiorelli}

\begin{abstract}
Introdução: A fibrilação atrial (FA) é uma complicação comum após a cirurgia cardíaca, sua frequência varia entre 25-40\% (MILLER et al., 2005). Embora sua etiologia seja incompletamente compreendida, mudanças estruturais dos átrios relacionadas à hipertensão, dano mecânico, sobrecarga de volume, idade, isquemia atrial intra-operatório, desequilíbrios eletrolíticos desempenham papel importante na patogênese da FA (AUER et al., 2005). Particularmente a hipomagnesemia, também foi identificada como preditora independente da FA pós-operatório (KOHNO et al., 2005). O magnésio (Mg) é um importante regulador de múltiplos processos cardiovasculares, incluindo a condução e contratilidade do miocárdio, fluxo de cálcio transmembrana, transporte de potássio, tônus do músculo liso vascular, reatividade coronariana e síntese de óxido nítrico (MANRIQUE et al., 2010). A importância do Mg na determinação da função cardiovascular é cada vez mais reconhecida, podendo influenciar no desempenho hemodinâmico, por seu efeito no tônus vascular, na modulação do cálcio intracelular, na regulação da atividade da catecolamina e seu papel essencial no metabolismo do trifosfato de adenosina. As propriedades antiarrítmicas do Mg são bem conhecidas e é amplamente reconhecida como adjuvante para o tratamento de arritmias.
\end{abstract}

Objetivo: Mostrar a importância da suplementação com sulfato de magnésio durante a cirurgia cardíaca como medida profilática contra a fibrilação atrial ou taquicardia juncional ectópica (JET) após cirurgia cardíaca em pacientes com cardiopatias congênitas.

Discussão: A administração de uma única dose de MgSO4 durante a CEC é uma prática mais segura e pode ter um efeito protetor no momento da reperfusão do miocárdio. Uma revisão da literatura mostra diversidade na dosagem e tempo de administração de magnésio para prevenção e reversão da FA.

Conclusão: Vários estudos descobriram que o enriquecimento do sangue com MgSO4 melhora os resultados hospitalares reduzindo a fibrilação atrial pós-operatória 


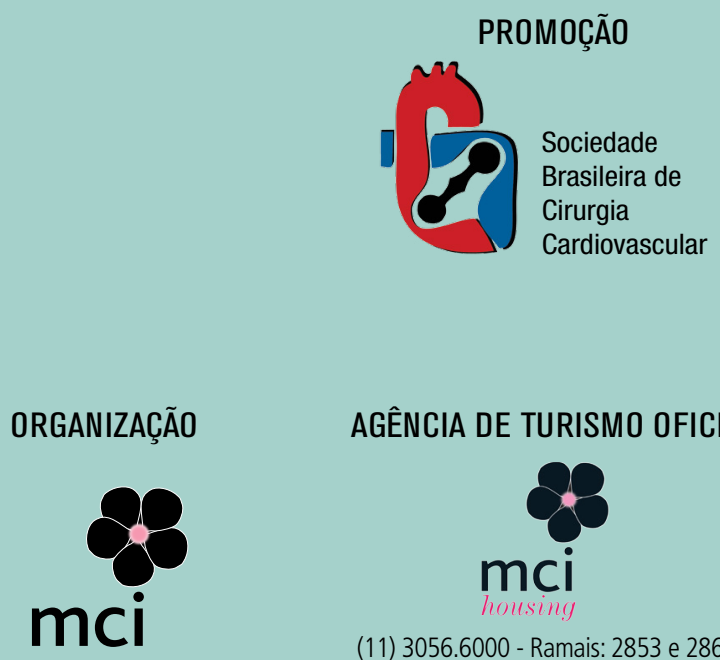

AGÊNCIA DE TURISMO OFICIAL

CIA AÉREA OFICIAL

513061.2959

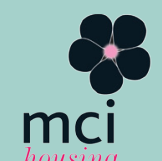

(11) 3056.6000 - Ramais: 2853 e 2860

atendimento@mcihousing.com.br

APOIO OFICIAL

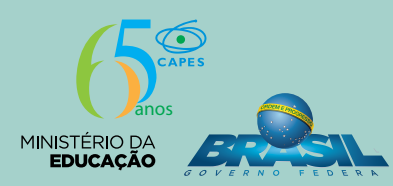

